\title{
SPECTROSCOPIC EXPLORATIONS OF MILKY WAY STELLAR POPULATIONS
}

\author{
Guillermo José Damke Calderón \\ La Serena, Chile
}

B.S., Universidad de La Serena, 2005

M.S., University of Virginia, 2011

A Dissertation Presented to the Graduate

Faculty of the University of Virginia

in Candidacy for the Degree of

Doctor of Philosophy

Department of Astronomy

University of Virginia

August 2016

Committee Members:

Steven R. Majewski

Phil Arras

Nitya Kallivayalil

Robin Garrod 
(c) Copyright by

\section{Guillermo José Damke Calderón}

All rights reserved

May 31, 2016 


\begin{abstract}
Stellar spectroscopy is a fundamental tool to investigate the dynamics and chemistry of stellar populations in the Milky Way. In this thesis, we present radial velocities and metallicities of giant stars in the disk and halo obtained through low-resolution spectroscopy $(R \sim 2,000-2,500)$ to undertake two focused studies.

Using spectroscopic data of M-giants, in addition to photometric and astrometric data, we have traced the tidal tails of the Sagittarius dwarf galaxy across the Northern and Southern Hemispheres. We compare these data to N-body simulations available in the literature to place constraints on the shape of the dark matter halo. As a critical tool in this enterprise, we present a modification to the near-IR calcium triplet metallicity determination method, which traditionally requires a priori knowledge of stellar distance to derive the stellar metallicities of RGB stars. The modified method provides a new tool to measure the metallicities of stars of unknown distances, and we apply it to stars along the tidal tails of Sagittarius.

In a separate study, we present measurements of the Solar motion and the Local Standard of Rest as derived from the observed kinematic of K-giant stars in the Grid Giant Star Survey.

Finally, we describe contributions made to the construction of the APOGEE-2S spectrograph, as a result of my work in the IR instrumentation team at the UVa Department of Astronomy.
\end{abstract}




\section{Acknowledgements}

This Ph.D. thesis is the materialization of a dream that I have had since I was a child: to become an astronomer. There are too many people that have been part of this process and that I would like to acknowledge.

First, I want to thank Dr. Steve Majewski, who has been my Ph.D. advisor. Undoubtedly, I have learned too many things from him as a person and as an astronomer. Steve, I will always be grateful to you for this.

I also want to thank the wonderful people of APOGEE and the IR lab at the University of Virginia. I have learned a lot working with the instrumentation team. I want to thank specially Dr. John Wilson and Dr. Jennifer Sobeck. Your support during these years has been very important for me. I also would like to thank Charles Lam. He is always willing to share some words with me and also help me with lab-related things. I also thank Matthew Nelson, Garrett Ebelke, Mita Tembe and Matthew Hall. Your help during this time has been very important to me.

I also thank Barbara Johnson, Janice Dean, Whitney Richardson and Jackie Harding. There is no question that all of you make the Department a better place.

I acknowledge the people of the SRM group. There are many former and current students in the group from whom I have learned a lot. I specially thank Ricky Patterson for all his help during my time at UVa.

I also want to acknowledge many other people that have been very important during my formation as a scientist. I acknowledge Dr. Rodolfo Barbá, Dr. Amelia Ramírez and Dr. Héctor Cuevas, who have been part of my formation during my undergraduate studies at Universidad de La Serena, Chile. I also want to acknowledge Dr. Armin Rest and Dr. Stella Kafka. They both supported me during the PIA program and the following years at Cerro Tololo, before I started my Ph. D. studies. 
Hugo Ochoa and Ciro Cortés have occupied a very special place in my heart during my career. I know that you both would be very proud and so happy to share this special time of my life with me. Thank you for all your support.

In addition, I want to thank my family and relatives. All of you have supported and encouraged me to pursue my childhood dream. I must thank my wife Pamela for her unconditional support during these years. Furthermore, our daughter Paula came to our lives during our time in Charlottesville. I also must acknowledge my parents and sisters. I thank them because they recognized my early interest in astronomy when I was a child. I acknowledge the Argentinean family, who have come to my life during my Ph.D. work. I also thank my uncles, aunts and cousins that have been there during my career. I keep an special place for those who have left us during these years that I have been far from home.

Finally, I want to acknowledge Fulbright-Chile and CONICYT for awarding me a Fulbright/CONICYT scholarship in 2008, and giving me the opportunity of pursuing my Ph.D. at the University of Virginia.

Guillermo. 


\section{Table of contents}

$\begin{array}{ll}\text { Abstract } & \text { ii }\end{array}$

1 Introduction 1

2 The Ca II Triplet as a Metallicity Indicator for Stars with Unknown

Distances 3

2.1 Background Information . . . . . . . . . . . . . . 3

2.2 The Ca II Triplet Equivalent Width . . . . . . . . . . . . . . . . 6

2.3 Cluster Data and CaT Metallicity Calibrations . . . . . . . . . 10

2.3.1 CaT Calibration Based on $K_{s, H B} \ldots \ldots \ldots \ldots \ldots \ldots \ldots$

2.3.2 CaT Metallicity Calibration Based on Isochrones . . . . . . . 15

2.3.3 Calibration Method Based on Colors . . . . . . . . . . . 19

2.4 Comparison of $[\mathrm{Fe} / \mathrm{H}]$ Results Among all Methods . . . . . . . . . 20

2.5 Conclusions . . . . . . . . . . . . . . . . . . . . . . . . 23

3 Tracing the Tidal Tails of the Sagittarius Dwarf using M Giant Stars 37

3.1 Introduction . . . . . . . . . . . . . . . 37

3.2 Data Acquisition, Reduction and Measurements . . . . . . . . . 39

3.2.1 Spectroscopic Target Selection . . . . . . . . . . . . . . . . 39

3.2.2 Spectroscopic Data Acquisition and Reduction . . . . . . . . . 41

3.2 .3 Radial Velocities Measurement . . . . . . . . . . . . . . . . . 43

3.2 .4 Giant/Dwarf Separation . . . . . . . . . . . . . . . . . 49

3.2.5 The Ca IITriplet Equivalent Width and Metallicity Estimation 79

3.3 Analysis and Results . . . . . . . . . . . . . . . . . . . 84

3.3.1 The tidal tails of Sagittarius traced by M-giants . . . . . . . 84

3.3.2 Comparison to the SDSS View and the Law \& Majewski (2010)

Model ... . . . . . . . . . . . . . . 87

3.3.3 GC3 Cell Counts and Orbital Plane Precession . . . . . . . . . 101

3.3.4 Metallicity Gradient Along the Tidal Arms . . . . . . . . . . 108

3.4 Conclusions . . . . . . . . . . . . . . . . . . . . . 111 
4 Solar Motion and the Local Standard of Rest as seen in the Grid Giant Star Survey 113

4.1 Introduction . . . . . . . . . . . . . . . . . . . . . . 113

4.2 The Grid Giant Star Survey . . . . . . . . . . . . . . . . 116

4.2 .1 Photometric Campaign . . . . . . . . . . . . . . . . 116

4.2.2 Low-Resolution Spectroscopic Campaign . . . . . . . . . . . 119

4.2.3 Radial Velocity Measurements . . . . . . . . . . . . . . 120

4.2.4 Metallicity Determination and Giant/Dwarf Discrimination . 121

4.2 .5 Estimation of Distances . . . . . . . . . . . . . . . . 127

4.3 Analysis . . . . . . . . . . . . . . . . . . . . . . . 130

4.3.1 Galactic Dynamical Model Description . . . . . . . . . . 130

4.3.2 Model Minimization and Results . . . . . . . . . . . . . 135

4.3.3 Model Tolerance to Distance and Radial Velocity Uncertainties 141

4.4 Conclusions and Future Work . . . . . . . . . . . . . . . 142

5 The APOGEE-S Spectrograph: Measurements and Laboratory Work150

5.1 Introduction . . . . . . . . . . . . . . . 150

5.1.1 The APOGEE Surveys in the Context of the Sloan Digital Sky

Survey . . . . . . . . . . . . . . . . 150

5.1 .2 The APOGEE Spectrographs . . . . . . . . . . . . . 154

5.2 Testing the VPH Grating for the APOGEE-S Spectrograph . . . . . . 157

5.2 .1 Motivation . . . . . . . . . . . . . . . . . . . 157

5.2 .2 Data Acquisition and Description . . . . . . . . . . . . 158

5.2 .3 Measurement Method and Results . . . . . . . . . . . . . . 162

5.3 The Plate Scale of the 2.54-m Irénée du Pont Telescope . . . . . . . . 175

5.3 .1 Motivation . . . . . . . . . . . . . . . . . . 175

5.3.2 Data Acquisition and Reduction . . . . . . . . . . . 177

5.3 .3 Data Modeling . . . . . . . . . . . . . . . . . . . . . 179

5.3.4 Results and On-Sky Check . . . . . . . . . . . . . . . 187

5.4 Thermal Blanket Fabrication . . . . . . . . . . . . . . . . . . . . . . . . 189

5.4 .1 Motivation. . . . . . . . . . . . . . . . . . . . 189

5.4.2 Design and Fabrication Process _. . . . . . . . . . . . 191

6 Summary and Future Work 201 


\section{Chapter 1}

\section{Introduction}

The study of our home galaxy has been crucial to the development of modern understanding of the Universe. Establishing the shape of the Galaxy, its size, and the location of the Sun in it, combined with a proper interpretation of the diffuse "Spiral Nebulae" observed by astronomers in the 18th century, provided essential clues towards understanding that the Galaxy is one among the myriad "Island Universes", which had been speculated by Thomas Wright and then by Immanuel Kant in the 18th century. Systematic observation of stars and stellar systems - including globular and open clusters, and satellites - within the MW, in addition to understanding of absorption by the interstellar medium, yield the clues to unveil physical properties of the Galaxy, and infer our place in it. For instance, it was Shapley (1918) who placed the Sun far from the center of the MW, by observing the distribution of globular clusters. Since the recognition that the shape of the Galaxy is dictated by orbits of stars within, the study of the Milky Way (MW hereafter) as a dynamical system has been among the more important topics in modern astronomy.

The new generation of large area, deep photometric surveys, complemented with spectroscopic studies of dynamics and chemistry, have changed the view of the MW 
dramatically. The halo is now known to host dwarf satellite galaxies, as well as many "tidal streams". The later are the relics of dwarf galaxies tidally disrupted by the MW gravitational potential. This formation scenario of the Galaxy was proposed by Searle \& Zinn (1978), and it is also a prediction of the Cold Dark Matter (CDM) model, where merging of numerous smaller systems form a larger system following a "hierarchical formation".

In this work, we use low-resolution spectroscopy $(R \sim 2,000-2,500)$ to measure radial velocities and derive chemical information of giant stars of the MW, and complement the spectroscopic data with matched photometric and astrometric data. In chapter 2 , we present a modification to the near-IR calcium triplet metallicity determination method to measure metallicities for stars with unknown distances. Chapter 3 presents a spectroscopic study of the tidal tails of the Sagittarius dwarf galaxy, which is the best example of tidal streams, known to date, generated by ongoing tidal disruption in the MW. In the same chapter 3, we apply the method presented in chapter 2 to derive metallicity information of the tidal tails. Chapter 4 presents preliminary results of the estimation of the Solar motion and the Local Standard of Rest measured using K-giants stars from the Grid Giant Star Survey. Chapter 5 presents part of my contribution to the construction of the APOGEE-2S spectrograph that I have made as a member of the IR instrumentation team at the UVa Department of Astronomy.

Each chapter contains its own introduction to the topic discussed in particular. 


\section{Chapter 2}

\section{The Ca Indicator for Stars with Unknown Distances}

\subsection{Background Information}

For almost three decades, the near-IR Ca II triplet (hereafter, CaT) $(\lambda 8498, \lambda 8542$

and $\lambda 8662 \AA$ ) has been used as a metallicity indicator for late-type giant stars. The strength of the lines of the triplet, combined with the intrinsic luminosity of Red Giant Branch (RGB hereafter) stars make this a convenient, efficient and powerful method to measure spectroscopic metallicities particularly for distant sources.

The method has its roots in the work of Armandroff \& Zinn (1988), who measured the strength of the CaT lines using integrated-light spectroscopy for a sample of 27 globular clusters with well-known metallicities. They showed that, in addition, there is a correlation between the CaT line strength and cluster metallicity.

Soon after, Olszewski et al. (1991) and Armandroff \& Da Costa (1991) showed 
that a linear correlation exists between the CaT strength (denoted as $\Sigma W$ ) and the magnitude of individual giant stars within each globular cluster. After establishing the stellar absolute magnitudes $M_{I}$ - using the $M_{V}(R R)-[F e / H]$ relation of Lee et al. (1990) for RR-Lyrae stars-, Olszewski et al. and Armandroff \& Da Costa showed that $\Sigma W$ followed a linear correlation with $M_{I}$ for the cluster RGB stars. In addition, the authors found that the locus of each cluster in the $\left(M_{I}, \Sigma W\right)$ plane varies with cluster metallicity. Finally, they showed that CaT strength is suitable for calculating metallicities of RGB stars in clusters, provided that $M_{I}$ is known.

Thus the strength of the CaT lines is a function of both metallicity and gravity for RGB stars. In fact, the CaT has also been used as a luminosity indicator for its sensitivity to gravity in integrated-light studies of stellar populations (e.g., Jones et al. 1984).

Therefore, if we want to use the CaT as a metallicity tool, we have to remove $\log g$ effects. Consequently, the CaT method relies on $M_{I}$ to counteract - and effectively remove - the effects of $\log g$ on $\Sigma W$ for the purpose of estimating metallicities. Nominally, this requires knowing the distance to the cluster to establish the absolute magnitude scale before metallicity can be estimated.

As an alternative, Armandroff \& Da Costa (1991) successfully explored the (V$\left.V_{H B}, \Sigma W\right)$ plane. This approach works because the absolute $\mathrm{V}$-magnitude of the Horizontal Branch (HB) is approximately constant among clusters, and adopting it as a reference point simplifies things greatly. First, the quantity $V-V_{H B}$ can be measured directly from the color-magnitude diagram (CMD hereafter) of each cluster even with only relative photometry. Second, the relative photometry does not need to be dereddened under the (reasonable) assumption that the reddening across the cluster is constant. That we can proceed with no knowledge of the distance or 
reddening to the cluster greatly simplifies the problem.

To implement their strategy, Armandroff \& Da Costa (1991) defined the "reduced equivalent width" ( $W^{\prime}$ hereafter) as the $\Sigma W$ value that a star of magnitude $V$ would have at $V=V_{H B}$. This is a simple but yet effective way to remove the effects of luminosity, temperature and surface gravity from the CaT line strength. As a result, values of $W^{\prime}$ can be compared directly between stars of different magnitudes. More importantly, $W^{\prime}$ correlates directly with $[\mathrm{Fe} / \mathrm{H}]$. $^{1}$

Most subsequent studies to determine metallicities from the CaT have followed the approach of Armandroff \& Da Costa (1991), by defining $W^{\prime}$, either in terms of absolute $V$ or $I$ magnitudes (Carrera et al. 2007), or apparent magnitudes with respect to the observed magnitude of the HB in the $V$-band $V-V_{H B}$ (Rutledge et al. 1997; Cole et al. 2004).

The CaT calibrations are defined empirically, and so typically restricted to a confined range of metallicity and age. The limits of both parameters are set by the span of the calibration clusters, and it is typical for new publications to continue to extend such limits. In addition, there exist different metallicity scales in use. Early publications usually adopted the metallicity scale for Galactic globular clusters of Zinn \& West (1984). This scale usually resulted in a non-linear $W^{\prime}$ versus $[\mathrm{Fe} / \mathrm{H}]$ behavior. However, Rutledge et al. (1997), in their seminal work, checked the relation between $W^{\prime}$ measurements for 71 Galactic globular clusters against the Zinn \& West (1984) and the Carretta \& Gratton (1997) metallicity scales. While they confirmed that the former scale resulted in a non-linear relation, the latter produces one that is linear.

\footnotetext{
${ }^{1}$ Note that, Olszewski et al. (1991) and Armandroff \& Da Costa (1991) explored the behavior of the CaT in the $\left((B-V)_{o}, \Sigma W\right)$ and $\left((V-I)_{o}, \Sigma W\right)$ planes, respectively. Both groups found that the cluster relations in these planes were not linear, so that it is preferable to develop calibrations against magnitudes, which are linear.
} 
In the last decade, new CaT metallicity calibrations have been defined using $K_{s}$ photometry. For example, Warren \& Cole (2009) (hereafter WC09) and Mauro et al. (2014) defined $W^{\prime}$ in terms of $K_{s}-K_{s, H B}$, while Carrera et al. (2013) did so in terms of absolute $M\left(K_{s}\right)$. The use of infrared photometry provides some advantages over optical wavelengths, namely, lower sensitivity to interstellar reddening and extinction, and the availability of large and homogeneous catalogs of stellar photometry and astrometry through, e.g., the Two Micron All-Sky Survey (2MASS hereafter; Skrutskie et al. 2006).

All CaT calibrations available in the literature require a quantity that provides some gauge of the distance to the cluster under study. This prerequisite has likely discouraged researchers from applying this technique on isolated stars where an absolute magnitude is unknown and no reference HB exists. In an attempt to circumvent this limitation, we have developed a set of CaT calibrations based on isochrones and 2MASS colors. Such calibrations do not require a priori knowledge of distance. Nevertheless, this new method enables metallicities to be gauged for isolated stars, as is the case for stars in the tidal tails of the Sagittarius dwarf galaxy that we study in Chapter 3.

In the following sections, we describe the procedure to measure equivalent widths, the calibration dataset, isochrones, calibration method, and results of recovering metallicities using the proposed calibrations.

\subsection{The Ca II Triplet Equivalent Width}

In this section, we introduce the "traditional" method employed to measure the equivalent width of the CaT. Even though we will not measure spectral lines for this chapter — we use literature data - we will use these definitions and methodology in Chap- 
ter 3. Therefore, we introduce these definitions here because the equivalent width measurement is relevant to the topics of the present Chapter.

The CaT lines are the main spectral features around 8,500 $[\AA]$ (Fig. 2.1) in A-M type stars. However, stars earlier than G3 are affected by the hydrogen Paschen series, whereas TiO become noticeable for stars redder than early-M types (see Cenarro et al. 2001, for a detailed description of this spectral region). In addition, this spectral region is free of telluric lines, whereas the continuum is relatively flat before $\mathrm{TiO}$ absorption features start to dominate from early-M stars and later.

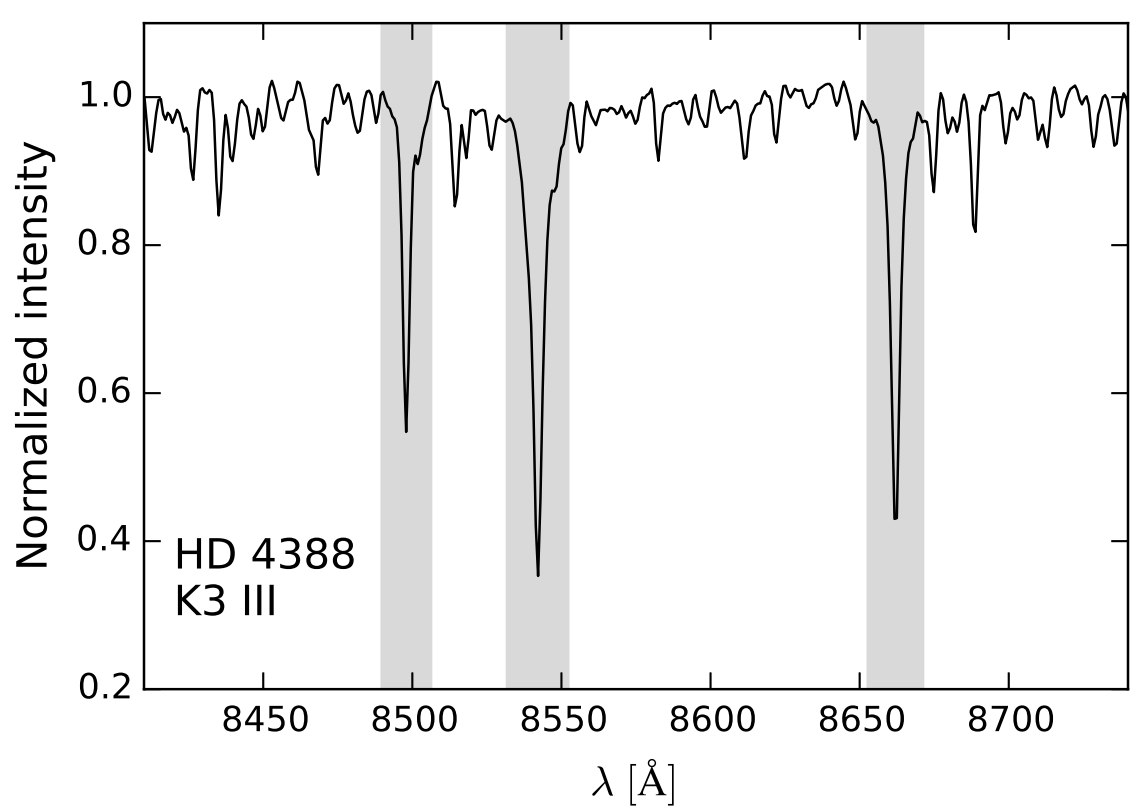

Fig. 2.1. - The near-IR Ca II triplet lines dominate the spectral region around $8500 \AA$, which is free of telluric features too. The shaded regions represent the spectral windows usually considered in equivalent width measurements of each line. The spectrum corresponds to the K3 III star HD 4388.

The strength of each spectral line is measured in terms of the equivalent width (hereafter, $W$ ), defined as: 


$$
W=\int_{\lambda_{1}}^{\lambda_{2}} \frac{1-f_{L}(\lambda)}{f_{C}(\lambda)} d \lambda
$$

where $f_{L}$ and $f_{C}$ are functions of wavelength that describe the profile of the spectral line and the continuum level, respectively. The integration limits $\lambda_{1}$ and $\lambda_{2}$ define the "line bandpass". Even though Armandroff \& Da Costa (1991) defined $f_{L}$ as a Gaussian, Cole et al. (2004) found that this does not describe the actual line profile for stars in the metal-rich half of their sample $([\mathrm{Fe} / \mathrm{H}] \approx-0.8)$, because the CaT lines exhibit broad damping wings that contain a large fraction of the line flux. Therefore, Cole et al. (2004) fitted each CaT line as the sum of a Gaussian and a Lorentzian profiles. We follow the same approach in Chapter 3, though we define and use the Lorentzian profile slightly differently to help the fitting routine to converge. We define the line fitting function as:

$$
f_{L}(\lambda)=f_{C}(\lambda)-A \exp \left[-\frac{1}{2}\left(\frac{\lambda-\lambda_{o}}{\sigma}\right)^{2}\right]-\frac{B}{1+\left(\frac{\lambda-\lambda_{o}}{\gamma}\right)^{2}}
$$

where the line flux $f_{L}(\lambda)$ at wavelength $\lambda$ is defined as the line continuum $f_{C}(\lambda)$, minus the Gaussian and Lorentzian components scaled by $A$ and $B$, respectively. Both components have a common line center $\lambda_{o}$, whereas $\sigma$ and $\gamma$ define the width of the Gaussian and Lorentzian component, respectively.

It is important to note that the continuum is affected by weak molecular lines and metallic lines that lower the true continuum level. Therefore, the estimated continuum level is a pseudo-continuum. Because of this, $W$ is usually referred to as the pseudo-equivalent width (Armandroff \& Zinn 1988).

The parameters in Equation 2.2 are usually solved by least-square minimization of the residuals between the spectral data and line model. For measuring our data on 
Sgr stars (Section 3.2.5), we wrote a Python language computer program where we:

- Doppler-correct the spectra to rest-frame wavelength by the measured heliocentric radial velocities (Section 3.2.3) and the relative observer motion with respect to the Sun. The latter quantity is obtained from the IRAF's task rvcorrect;

- Fit $f_{L}$ and $f_{C}$ to the line and continuum data, respectively. These data intervals are defined by line and a continuum bandpasses, respectively. Table 2.1 lists the bandpasses for the three CaT features. These bandpasses effectively mask out the spectrum everywhere except for the spectral line, as well as a blue and a red continuum around the spectral feature, respectively. Figure 2.2 shows the actual wavelength ranges of bandpasses, and an example of the best-fit for a spectrum of HD 4388. The code performs the data fits following a Levenberg-Marquadt algorithm implemented through the Imfit library for Python. Because the spectra are normalized, we could fix $f_{C}=1$. However, a linear fit to the continuum produces smaller residuals for the best-fit values.

- Finally, calculate $W$ by integrating Equation 2.1 within the corresponding line bandpass.

Table 2.1. Ca II Triplet wavelengths, line and continuum bandpasses (in $\AA$ ).

\begin{tabular}{cccc}
\hline \hline Line Center & Line Bandpass & Blue Continuum & Red Continuum \\
\hline 8498.02 & $8490-8506$ & $8474-8489$ & $8521-8531$ \\
8542.09 & $8532-8552$ & $8521-8531$ & $8555-8595$ \\
8662.14 & $8653-8671$ & $8626-8650$ & $8695-8725$ \\
\hline
\end{tabular}



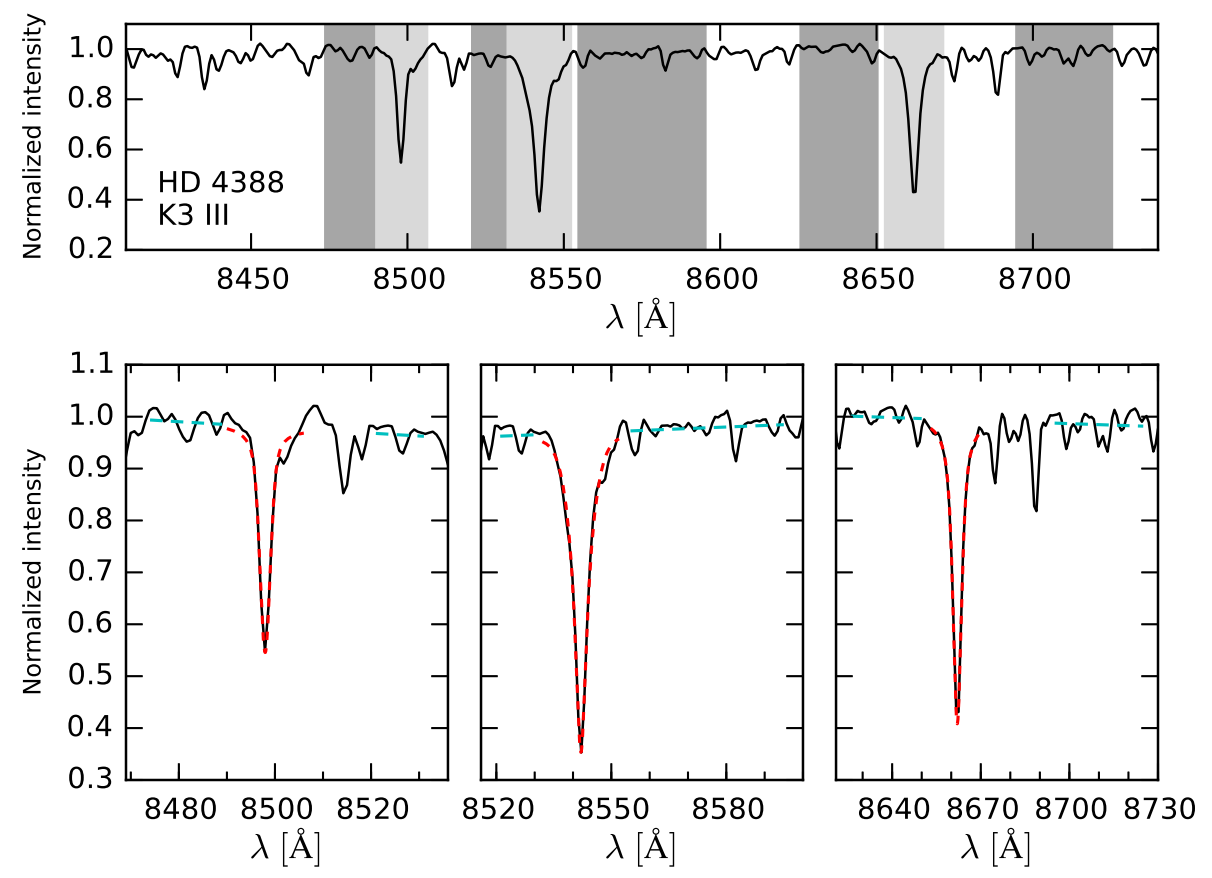

Fig. 2.2.- Top: The CaT features and the respective bandpasses for EW measurement. Line bandpasses are marked in light gray, whereas continuum bandpasses appear in darker gray. Each line has a blue (left) and red (right) continuum bandpass. Bottom: The best-fit of the line profile (red) and continuum (cyan) for each CaT feature. The spectrum corresponds to the star HD 4388 (K3 III).

\subsection{Cluster Data and CaT Metallicity Calibrations}

The calibration of our metallicity procedure is based fundamentally on the work and cluster data of WC09 work and cluster data, however we begin by redoing their calibration. Nevertheless, we discuss their work as published because introduce the calibration rationale that we will follow later for our modified method, and we obtain metallicity values for stars and clusters using exactly the same computational routines for all calibrations. After, we will use these metallicities derived following WC09 "traditional" CaT method — based on the magnitude of the HB - to assess the scatter of the new calibrations that we will develop. 


\subsubsection{CaT Calibration Based on $K_{s, H B}$}

An important assumption in CaT calibrations is that all stars in a cluster belong to a single population. As a consequence, stars in a particular cluster will have the same metallicity.

WC09 extended the CaT system into the near-IR $K_{s}$ bandpass of 2MASS. This calibration uses the red clump/horizontal branch magnitude $K_{s, H B}$ at the RR-Lyrae instability strip as its reference. The sample of calibrators comprises 17 clusters (Table 1 in WC09), where ten of these were initially published by Cole et al. (2004) (C04, hereafter).

Even though some authors have published CaT calibrations based on $K_{s}$ photometry, the data published by WC09 includes 2MASS IDs for the stars that they selected as cluster members (their Tables 2 and 3). The WC09 data also include $K_{s}$ magnitudes, radial velocities, $\Sigma W$ and uncertainties in the measurements.

Using the 2MASS IDs, we obtained 2MASS $J, H, K_{s}$ photometry for the WC09 stars. We only kept those stars whose 2MASS photometric quality flag in each band is " $\mathrm{A} " 2$. We dereddened the data following the extinction laws of Indebetouw et al. (2005), with $A_{V} / A_{K} \sim 8.8$ and $R_{V} \sim 3.1$ from Cardelli et al. (1989).

We adopted $E(B-V)$ values from the 2010 version of Harris (1996) and the $\mathrm{WEBDA}^{3}$, for globular and open clusters, respectively. The adopted absorption relations for each band are:

$$
A_{J}=2.5 A_{V} / 8.8
$$

\footnotetext{
${ }^{2}$ MASS provides a summary of the quality of each band through "quality flags". The "A" flag indicates that the photometry has signal-to-noise ratio $>10$ and a valid error estimate in the respective band.

${ }^{3}$ http://webda.physics.muni.cz/
} 


$$
A_{H}=1.55 A_{V} / 8.8
$$

$$
A_{K_{s}}=A_{V} / 8.8
$$
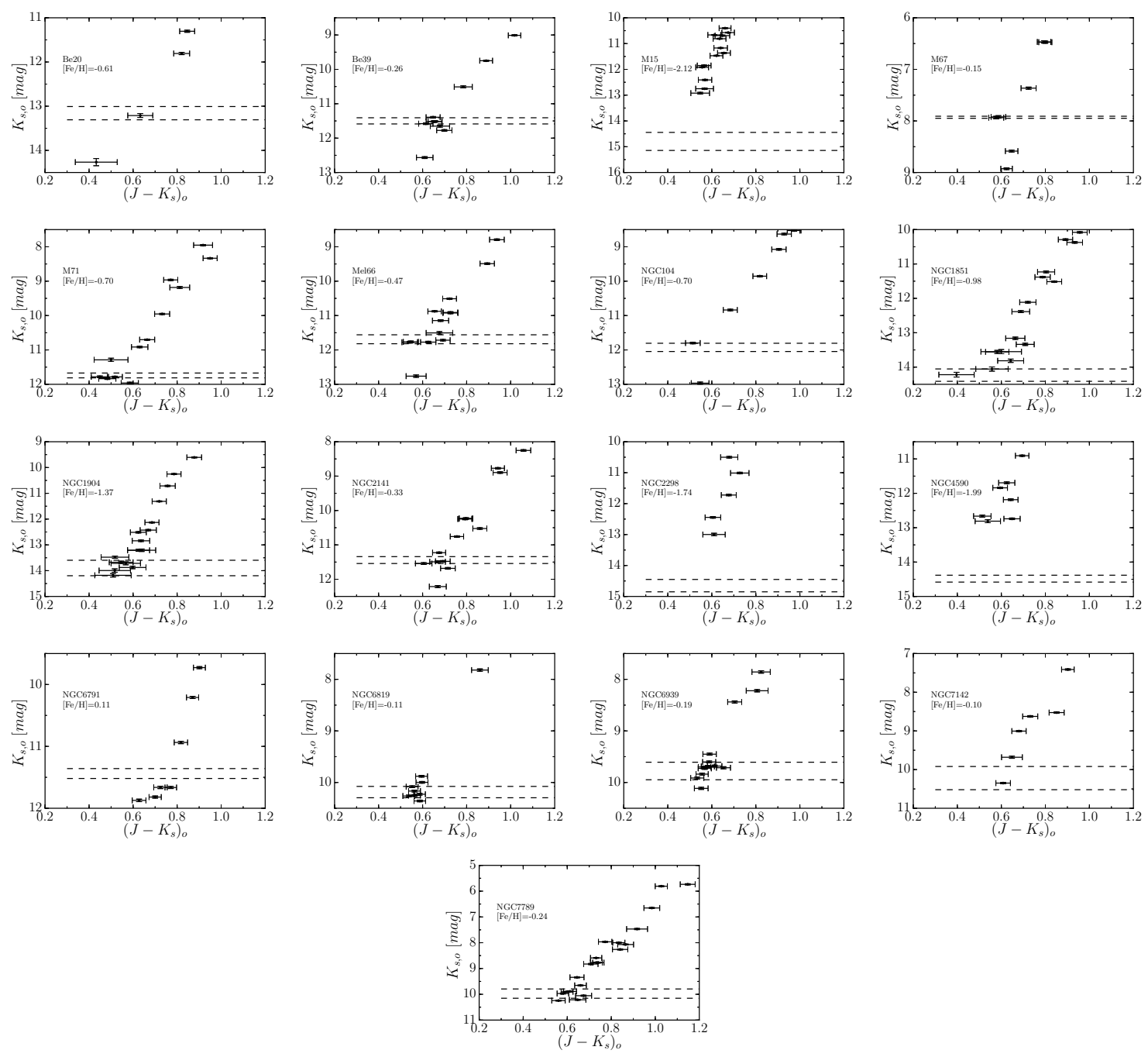

Fig. 2.3.- $\left(J-K_{s}\right)_{o}$ versus $K_{s, o}$ color-magnitude diagrams for the calibration clusters. Horizontal lines show the $K_{s, o}$ magnitude and uncertainty of the horizontal branch listed by WC09.

Figure 2.3 shows the dereddened color-magnitude diagrams for the RGBs of all cal- 
ibration clusters. We only accepted spectroscopic measurements from Table 3 of WC09 flagged as "accepted star" 4 into the calibration, and we included the whole set of stars from their Table 4.

WC09 use the metallicity scales of Carretta \& Gratton (1997) and Friel et al. (2002), for globular and open clusters, respectively. These scales should form an homogeneous metallicity scale, and reduce the uncertainty of the derived metallicity calibration. At the same time, the cluster sample spans a large range in age $(2.5-$ 12.6 Gyr $)$ and $(-2.12 \leq[\mathrm{Fe} / H] \leq+0.11)$ dex.

WC09 define $\Sigma W$ as the linear combination of $W$ of the three Ca II lines with equal weights:

$$
\Sigma W=W_{8498}+W_{8542}+W_{8662},
$$

where the fitting function, as well as the line and continuum bandpasses follow the definitions given in the previous subsection.

We should note that the definition of $\Sigma W$ changes among authors. For instance, Armandroff \& Da Costa (1991) define it as the sum of the two strongest CaT lines, because including the third line increases the uncertainty in $\Sigma W$. Rutledge et al. (1997) and Carrera et al. (2007) have shown that the $\Sigma W$ values can easily be transformed between different systems.

Figure 2.4 shows the $\left(\Sigma W, K_{s}-K_{s, H B}\right)$ plane for each cluster in the sample. As mentioned before, stars of each cluster follow a linear relation in this plane because they have the same metallicity, but the locus of each cluster in this plane shifs as a function of the cluster metallicity. Clusters with higher metallicities have larger $\Sigma W$ values for a given $K_{s}-K_{s, H B}$, and so the trends appear shifted upward.

\footnotetext{
${ }^{4}$ WC09 use this flag for stars accepted as cluster members.
} 
In this plane, we define the reduced equivalent width, $W^{\prime}$, of a cluster as:

$$
W^{\prime}=\Sigma W_{j}-\beta\left(K_{s, j}-K_{s, H B}\right)
$$

where $\Sigma W_{j}$ and $K_{s, j}$ correspond to the values of $\Sigma W$ and $K_{s}$ for the $j$-th star in the cluster, where $K_{s, H B}$ is the HB magnitude.

We fit Equation 2.7 to each cluster in the sample to solve for $\beta$ and $W^{\prime}$, with the constraint that the slope $\beta$ is the same for all clusters. For optimal results, we therefore fit all clusters simultaneously. We use the "Orthogonal Distance Regression" algorithm (ODR hereafter) implemented in the Scipy library in Python. ODR properly accounts for uncertainties in both the dependent and independent variables. Uncertainty for $\Sigma W$ comes from the WC09 measurements, whereas the uncertainty in $\left(K_{s}-K_{s, H B}\right)$ is $\left[\left(\sigma\left(K_{s}\right)\right)^{2}+\left(\sigma\left(K_{s, H B}\right)\right)^{2}\right]^{1 / 2}$. The first and second terms in the last expression are the uncertainties in 2MASS photometry and in the magnitude of the $\mathrm{HB}$, respectively. We also tried the fitting method presented by Rutledge et al. (1997), but found that differences in the results from both methods are negligible. Because of this, we prefer the ODR routine because it is a more standard procedure. The dashed line in each panel of Figure 2.4 represents the fit solution. By definition, $W^{\prime}$ is the value, from the fitted relation, that $\Sigma W$ has at $\left(K_{s}-K_{s, H B}\right)=0$.

The final step in this calibration solves for the relation between the value of $W^{\prime}$ and literature metallicity values. We plot the $\left(W^{\prime},[\mathrm{Fe} / \mathrm{H}]\right)$ data in the left panel of Figure 2.5. We fit a linear and a quadratic relations using ODR to include uncertainties in $W^{\prime}$ and $[\mathrm{Fe} / \mathrm{H}]$. The difference between both fits is negligible, and the trend is clearly linear. 


\subsubsection{CaT Metallicity Calibration Based on Isochrones}

WC09 showed, and we verified above, that the 2MASS $K_{s}$ band is appropriate for defining $W^{\prime}$ using a linear relation, with $K_{s, H B}$ as the zero-point. Armed with this, it is possible to use the CaT as a means to determine metallicities for cluster RGB stars. However, use of such a relation requires that the $K_{s}$ magnitude of the horizontal branch $K_{s, H B}$ to be known, the usefulness of the method for field/isolated RGB stars is minimal unless we know the distance to the objects. This limitation ignited our interest for developing an alternative method that could overcome this problem. One option that we explored utilizes isochrones to obtain synthetic $M\left(K_{s}\right)$ absolute magnitudes, given the stellar $(J-K s)_{o}$ color from derived photometry. Below, we introduce the isochrone set that we will use to develop this idea.

\section{Isochrone Set}

We downloaded PARSEC (version 1.1) isochrones (Bressan et al. 2012) for 3, 5, 8, 10 and 12 Gyr. These isochrones include synthetic magnitudes for 2MASS $J, H$ and $K_{s}$ bandpasses. For each age, we obtained sequences of isochrones with metal content $Z$ ranging from $Z_{\min }$ to $Z_{\max }$ in steps of size $\Delta Z$, through the "CMD 2.5 input form" web interface ${ }^{5}$. The ranges used are listed in Table 2.2. These sequences were then concatenated to generate one table of isochrones for each age. Each table of isochrones has $0.0001 \leq Z \leq 0.06\left(-2.18 \leq[\mathrm{Fe} / H] \leq+0.59\right.$, for $\left.Z_{\odot}=0.0152\right)$.

\section{Methodology and Calibration}

In brief, the calibration procedure follows the same main steps that we described in Section 2.3.1, except that we replace the $\left(K_{s}-K_{s, H B}\right)$ term by a synthetic absolute

\footnotetext{
${ }^{5}$ http://stev.oapd.inaf.it/cgi-bin/cmd
} 
magnitude $M\left(K_{s, i s o}\right)$ that we obtain from isochrones, given the RGB star's color.

In the first step, we obtain the $K_{s}$ absolute magnitudes $\left(M\left(K_{s, i s o}\right)\right.$ hereafter $)$ from the RGB track of the PARSEC isochrone that corresponds to the dereddened $\left(J-K_{s}\right)_{o}$ color of the stars and that matches the literature metallicity of the cluster. If necessary, we apply a linear interpolation in color and metallicity, because isochrones are composed of discrete points. We believe that this simple interpolation does not introduce noise, because the grid sampling in color and metallicity is very fine.

We use isochrones of a single fixed age for all clusters. We will show that the age of the isochrone set does not affect the metallicities derived through this method. In fact, this may not be surprising. For instance, Carrera et al. (2007) showed that age has a small effect on the CaT calibrations, at least for clusters older than 0.25 Gyr (the effect becomes larger for younger clusters). This 0.25 Gyr lower limit is much younger than the stars of Sagittarius that we intend to measure in Chapter 3.

In the second step, we follow the same fitting procedure as for Equation 2.7. However, now we measure the slope $\beta$ for the locus of each cluster in the $\left(M\left(K_{s, i s o}\right), \Sigma W\right)$ plane, instead of $\left(K_{s}-K_{s, H B}, \Sigma W\right)$. In thus case, the reduced equivalent width is:

$$
W^{\prime}=\Sigma W_{j}-\beta M\left(K_{s, i s o, j}\right)
$$

We solve the system of equations following the same prescription as for Equation 2.7,

Table 2.2. Isochrone intervals

\begin{tabular}{lll}
\hline \hline$Z_{\min }$ & $Z_{\max }$ & \multicolumn{1}{c}{$\Delta Z$} \\
\hline 0.0001 & 0.0005 & 0.00002 \\
0.0005 & 0.001 & 0.0001 \\
0.001 & 0.005 & 0.0002 \\
0.005 & 0.01 & 0.001 \\
0.01 & 0.06 & 0.002 \\
\hline
\end{tabular}


and we derived uncertainties, $\sigma_{M\left(K_{s, i s o}\right)}$, from the photometric uncertainty in $\left(J-K_{s}\right)_{o}$ by calculating the mean deviation around $M\left(K_{s, i s o}\right)$ for $\left(J-K_{s}\right)_{o} \pm \sigma_{\left(J-K_{s}\right)_{o}}$.

We repeated the fitting procedure for the five ages in the set of isochrones. The best-fit values for $\beta$ and the standard deviation, reported by ODR, are listed in the second column of Table 2.3. Figure 2.6 illustrates the fit results in the $\left(M\left(K_{s, i s o}\right), \Sigma W\right)$ plane for $8 \mathrm{Gyr}$ isochrones. It can be seen that the clusters follow linear sequences. The latter holds true for the five sets of isochrones of different age that we use in this calibration. There is scatter in the sequences for the clusters, which may have been introduced by the adoption of $M\left(K_{s, i s o}\right)$. In any case, the scatter around the linear trend in this plane is slightly larger than the intrinsic scatter seen for $\left(K_{s}-K_{s, H B}\right)$, which uses only photometric data; although uncertainties in $M\left(K_{s, i s o}\right)$ are larger than in $\left(K_{s}-K_{s, H B}\right)$.

Next, we derive the $W^{\prime}-[\mathrm{Fe} / \mathrm{H}]$ relation by fitting the isochrone-based $W^{\prime}$ and literature metallicities. Again, we use the ODR routine to take the uncertainties in both variables into account, which are the $1-\sigma$ values for $W^{\prime}$ reported by ODR from the fit in the previous step. The upper right and lower panels of Figure 2.5 shows the $W^{\prime}$ results for the calibration clusters compared to literature metallicities, besides the best-fit for linear and quadratic relations. These results can be compared to the

Table 2.3. Best-fit coefficients for the CaT calibration based on isochrones. We list results for isochrones of five different ages.

\begin{tabular}{ccccccc}
\hline \hline $\begin{array}{c}\text { Isochrone Age } \\
{[\text { Gyr }]}\end{array}$ & $\beta$ & \multicolumn{2}{c}{$a$} & $b$ & \multicolumn{2}{c}{$A$} \\
{$[F e / H]=a \times W^{\prime}+b$} & \multicolumn{2}{c}{$\begin{array}{c}B \\
{[F e / H]}\end{array}=A \times W^{\prime 2}+B \times W^{\prime}+C$} \\
\hline 3.0 & $-0.430 \pm 0.027$ & $0.361 \pm 0.013$ & $-2.691 \pm 0.075$ & $-0.001 \pm 0.008$ & $0.370 \pm 0.075$ & $-2.710 \pm 0.168$ \\
5.0 & $-0.416 \pm 0.026$ & $0.364 \pm 0.013$ & $-2.778 \pm 0.075$ & $-0.003 \pm 0.008$ & $0.395 \pm 0.078$ & $-2.840 \pm 0.176$ \\
8.0 & $-0.411 \pm 0.025$ & $0.364 \pm 0.013$ & $-2.820 \pm 0.076$ & $-0.003 \pm 0.008$ & $0.394 \pm 0.079$ & $-2.881 \pm 0.183$ \\
10.0 & $-0.409 \pm 0.025$ & $0.363 \pm 0.013$ & $-2.836 \pm 0.075$ & $-0.003 \pm 0.008$ & $0.392 \pm 0.079$ & $-2.896 \pm 0.184$ \\
12.0 & $-0.408 \pm 0.025$ & $0.363 \pm 0.013$ & $-2.847 \pm 0.075$ & $-0.003 \pm 0.008$ & $0.392 \pm 0.079$ & $-2.909 \pm 0.185$ \\
\hline
\end{tabular}


upper left panel, which shows the calibration referenced to the HB from the previous section. It is clear that $W^{\prime}$ from the isochrone-based method also follows a linear correlation with metallicity.

\section{Calculation of Metallicities Through a Calibration Based on Isochrones}

With an isochrone-based CaT calibration in place, we should be able to measure metallicities for stars with unknown distances but known $\left(J-K_{s}\right)_{o}$ color. However, the value of $M\left(K_{s, i s o}\right)$ itself depends on metallicity and age. We simplify the problem by showing that the age dependency is removed by the calibration. With the age dependence removed from consideration, the prescription for ascertaining the metallicity of an RGB star amounts to a simple iterative process: Hence, the approach that we propose is a self-consistent solution: We seek the input $M\left(K_{s, \text { iso }}\right)$ value, derived from an isochrone with metallicity $[\mathrm{Fe} / \mathrm{H}]_{i s o, i n}$ for the star's measured $\left(J-K_{s}\right)_{o}$, such that the metallicity derived from the CaT calibration $[\mathrm{Fe} / \mathrm{H}]_{\text {iso out }}$ is equal to $[\mathrm{Fe} / \mathrm{H}]_{i s o, i n}$. It is possible to find a non-degenerate solution to this iterative quest because the $[\mathrm{Fe} / \mathrm{H}]_{\text {iso,out }}-[\mathrm{Fe} / \mathrm{H}]_{\text {iso,in }}$ value almost always decreases monotonically with $[\mathrm{Fe} / \mathrm{H}]_{i s o, i n}$. As a result, there is one and only one value where $[\mathrm{Fe} / \mathrm{H}]_{\text {iso }, \text { out }}=[\mathrm{Fe} / \mathrm{H}]_{\text {iso, in }}$, which is the value that we consider as the metallicity of the star. We illustrate this in Figure 2.7. A major bonus is that the method also gives $M\left(K_{s, i s o}\right)$ for the star.

We implemented this technique via programs in the Python language. The first program, EZ_CHRONES, simplifies reading and interpolating data from isochrones given a set of input parameters. These isochrones can have data columns from different photometric systems or stellar parameters, and the user can specify combinations of input and output columns. In this case, we use it to read 2MASS absolute magnitudes 
from a set of single-age isochrones, given an input color and a set of multiple values of metallicity. Then, a second routine takes an array of output values for $[\mathrm{Fe} / \mathrm{H}]_{i s o, i n}$ and $K_{s, i s o}$ from EZ_CHRONES, given the input $\left(J-K_{s}\right)_{o}$ and isochrone age, loads the parameters of the CaT calibration, and calculates $W^{\prime}$ and $[\mathrm{Fe} / \mathrm{H}]_{i s o, o u t}$ for each input metallicity. Finally, this second code iteratively solves for the metallicity value where $[\mathrm{Fe} / \mathrm{H}]_{i s o, \text { out }}=[\mathrm{Fe} / \mathrm{H}]_{i s o, i n}$. The code applies a linear interpolation in metallicity if the solution lies in between of two data points. The right panel of Figure 2.7 shows an example of this process. Note how isochrones of different ages produce basically the same metallicity results. We test the uncertainty of the results produced by this method in Section 2.4.

\subsubsection{Calibration Method Based on Colors}

After successfully developing a CaT-based W'-[Fe/H] calibration based on $\left(J-K_{s}\right)_{o}$ and isochrones, it seems reasonable to wonder if a color-based CaT calibration is possible. This approach would not require isochrones, skipping the intermediate step of calculating $M\left(K_{s, i s o}\right)$ and the iteration required to derive a metallicity. Therefore, we explore the behavior of the CaT in the $\left(\left(J-K_{s}\right)_{o}, \Sigma W\right),\left((J-H)_{o}, \Sigma W\right)$, and $\left(\left(H-K_{s}\right)_{o}, \Sigma W\right)$ planes. In the three cases, we repeat the calibration steps listed in Section 2.3.1. We plot the cluster data and the best-fit relations in figures 2.8, 2.9 and 2.10 .

Again, we solve for the best-fit parameters with ODR. We calculate uncertainties in color as the square root of the sum in quadrature of the respective uncertainties in 2MASS photometry for each star. Finally, we calculate $\left(W^{\prime},[\mathrm{Fe} / \mathrm{H}]\right)$ relations for the three color combinations. The results are shown in Figure 2.11, where we find that the results are comparable to the ones from the isochrone-based method. 


\subsection{Comparison of $[\mathrm{Fe} / \mathrm{H}]$ Results Among all Meth- ods}

In this section, we assess the quality of the new CaT calibrations that we have introduced in this chapter.

First, we calculate metallicities for every star in the calibration clusters, using the five calibrations discussed: $\left(K_{s}-K_{s, H B}\right), M\left(K_{s, i s o}\right),\left(J-K_{s}\right)_{o},(J-H)_{o}$ and $\left(H-K_{s}\right)_{o}$

We then compare these metallicities against the literature values that we used for the calibration. In Figure 2.12, we present histograms (a) and plots (b) of these results. The histograms (Fig. 2.12a) show the difference between the metallicity of individual stars and the cluster literature values. Thus, these histograms give some sense of the systematic and random uncertainties associated with each method, parameterized by the mean and standard deviation of the histograms. Note that we include the histogram for CaT results for the $\left(K_{s}-K_{s, H B}\right)$ calibration. In particular, this histogram shows that there is an intrinsic scatter in the "traditional" CaT method of $\sim 0.1$ dex, although it seems to be the most precise method among the five calibrations. However, all methods have an average difference with respect to the literature values very close to zero, and standard deviations no greater than 0.25 dex. Figure $2.12 \mathrm{~b}$ shows plots for the same metallicity measurements, but we calculate a weighted-average and standard deviation for each cluster and then subtract the literature metallicity. The weights are assigned as the inverse of the square of uncertainty in metallicity for each star.

For all calibrations, we see no significant trends with metallicity. As expected, the method based on the HB exhibits the smallest scatter, whereas the other four 
methods have scatter of similar magnitude among them. However, we see that some clusters show larger differences compared to the literature values, typically those close to the metal-rich end of the calibration. We investigate possible causes for this below.

Next, we attempt to quantify the uncertainty introduced by the newly developed methods alone by assuming the intrinsic scatter of the CaT represents the true intracluster metallicity scatter. Of course, this is an extreme assumption, but gives some idea of the true intrinsic scatter introduced by our methodology. To do thus, we compare the CaT metallicities obtained using the four calibrations that we developed against the values obtained from the HB-based calibration in Figure 2.13. The histograms show the difference in derived metallicity for each star compared to that from the HB-based CaT value. As expected from Figure 2.12, the mean values lie very close to zero. The standard deviations give some idea about the scatter inherent to each method. All of them have an intrinsic 0.15-0.2 dex uncertainty. The figure also shows plots of the average differences of mean metallicity values compared to metallicity values from the HB-based method. The deviations are smaller than 0.25 dex in all cases. However, the scatter that we saw on the metal-rich end in Figure $2.12 \mathrm{~b}$ is also present in these plots.

To understand what may be causing the deviations at high metallicity, we tested whether cluster age and dereddening may affect the scatter in the values derived. Figure 2.14 shows the average metallicity differences between the literature and the HB-based CaT metallicities with clusters, color coded by age. Figure 2.15 show the same, but color-coded by $E(B-V)$. Inspection of Figure 2.14 shows that the clusters with high scatter (with $-0.5<[\mathrm{Fe} / \mathrm{H}]<0$ ) are also younger than $\sim 6-7$ Gyr. However, Figure 2.15 show that these clusters also have higher reddening values.

Finally, we test our new calibrations measuring clusters with unknown metallicity. 
To do so, we use the data for five clusters published by WC09. These objects had no literature values before WC09 measured them; therefore, they were not included in the sample of calibrators.

We calculate the weighted mean metallicity value per cluster using the five methods, and we take the HB-based CaT results as the reference value to estimate the deviations. Figure 2.16 shows the results color-coded by age and $E(B-V)$. The clusters in this sample have ages between 1.0 and $5.0 \mathrm{Gyr}$, and some of them are highly reddened. Results for three clusters lie within 0.1 dex of the HB-based method for the four calibrations that we have developed, and the three clusters span almost the whole age range of this test dataset. On the other hand, two clusters show large differences. Even though these are the two youngest objects in the dataset, they are the most reddened as well.

From the evidence, it is likely that the erroneous metallicity results are due to incorrect reddening estimations. First, we have shown that isochrones of different ages produce almost identical metallicity results. For instance, the metallicities of the two youngest clusters in the calibration data, estimated through the new CaT calibrations, exhibit almost no difference when compared to the HB-based CaT values. Additionally, the color-based methods are independent of age and exhibit the same linear trend in the $\left(W^{\prime},[\mathrm{Fe} / \mathrm{H}]\right)$ plane as the other calibrations - although age could have an impact in the scatter for these relations because the position of the RGB in the CMD depends mildly on age. On the contrary, an over (under) correction in reddening value will result in an over (under) estimate of $W^{\prime}$ for the star. As a result, we will measure an over (under) estimated metallicity. 


\subsection{Conclusions}

We have developed and tested a new approach to measure stellar metallicities for isolated $R G B$ stars through use of the measured CaT values and dereddened colors and a set of adopted isochrones, and effectively removing the dependence on distance or calibration of absolute magnitude that the traditional $\mathrm{CaT}$ approaches require.

These new calibrations have an inherent uncertainty of $0.15-0.2$ dex compared to the nominal "cluster" CaT method. We have shown, for the calibrations that use isochrones, that a single adopted isochrone age can be used to derive metallicities for the whole age range spanned by the clusters, because the age of the set of isochrones does not affect the derived metallicities. However, as might be expected, there is evidence that erroneous dereddening is a limitation of the method. Nevertheless, the method is very functional for high latitude RGB stars, and the scatter that results from the proposed calibrations are completely acceptable for our purposes. The derived method opens the door to exploring a number of problems in Galactic astronomy. We exploit it for Sgr tidal tails, where HB-based methods are useless. 

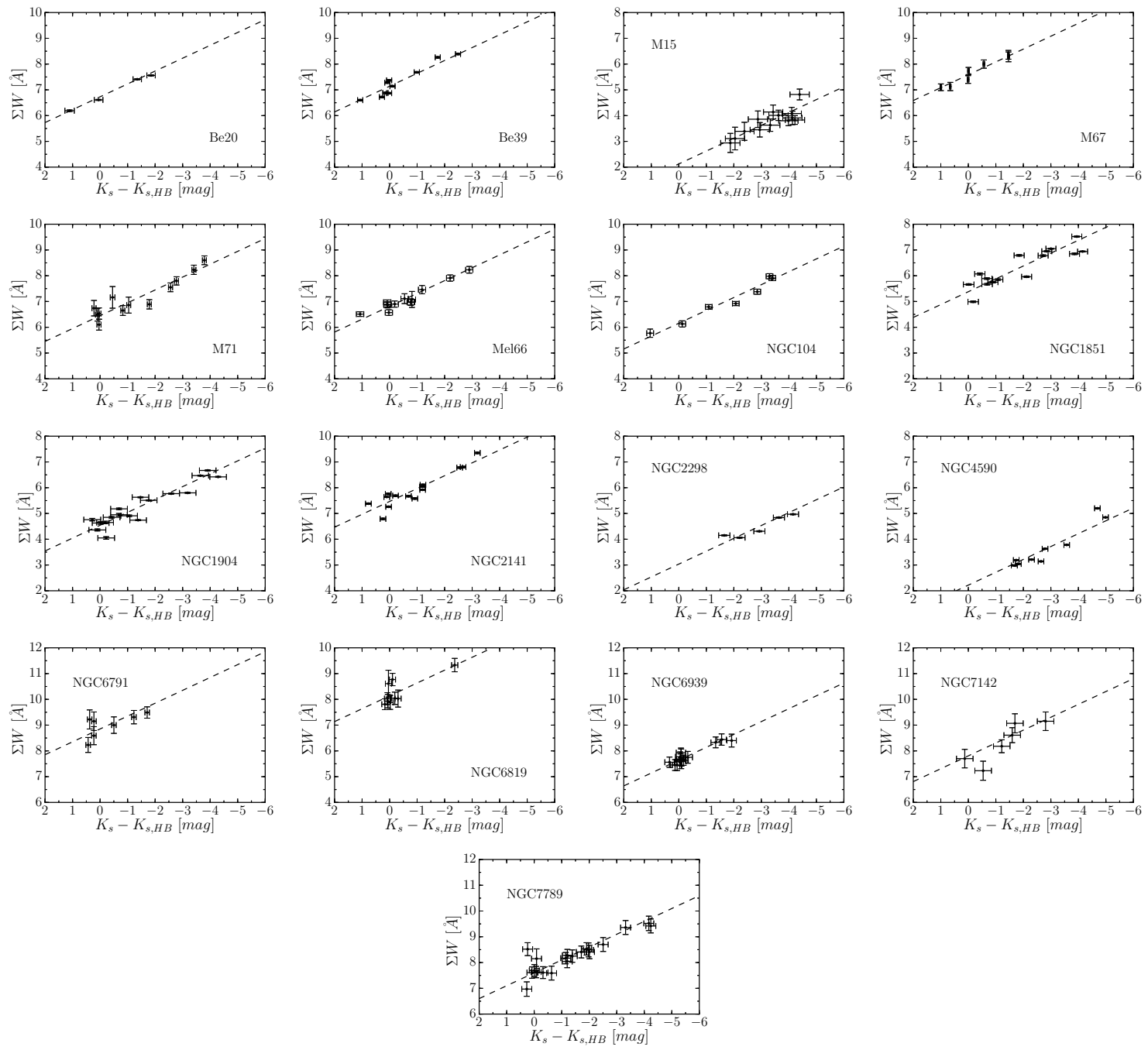

Fig. 2.4. - The calibration clusters in the $\left(\Sigma W, K_{s}-K_{s, H B}\right)$ plane. The dashed line in each panel represents the best linear fit with a single-valued slope $\beta$ for all clusters. 

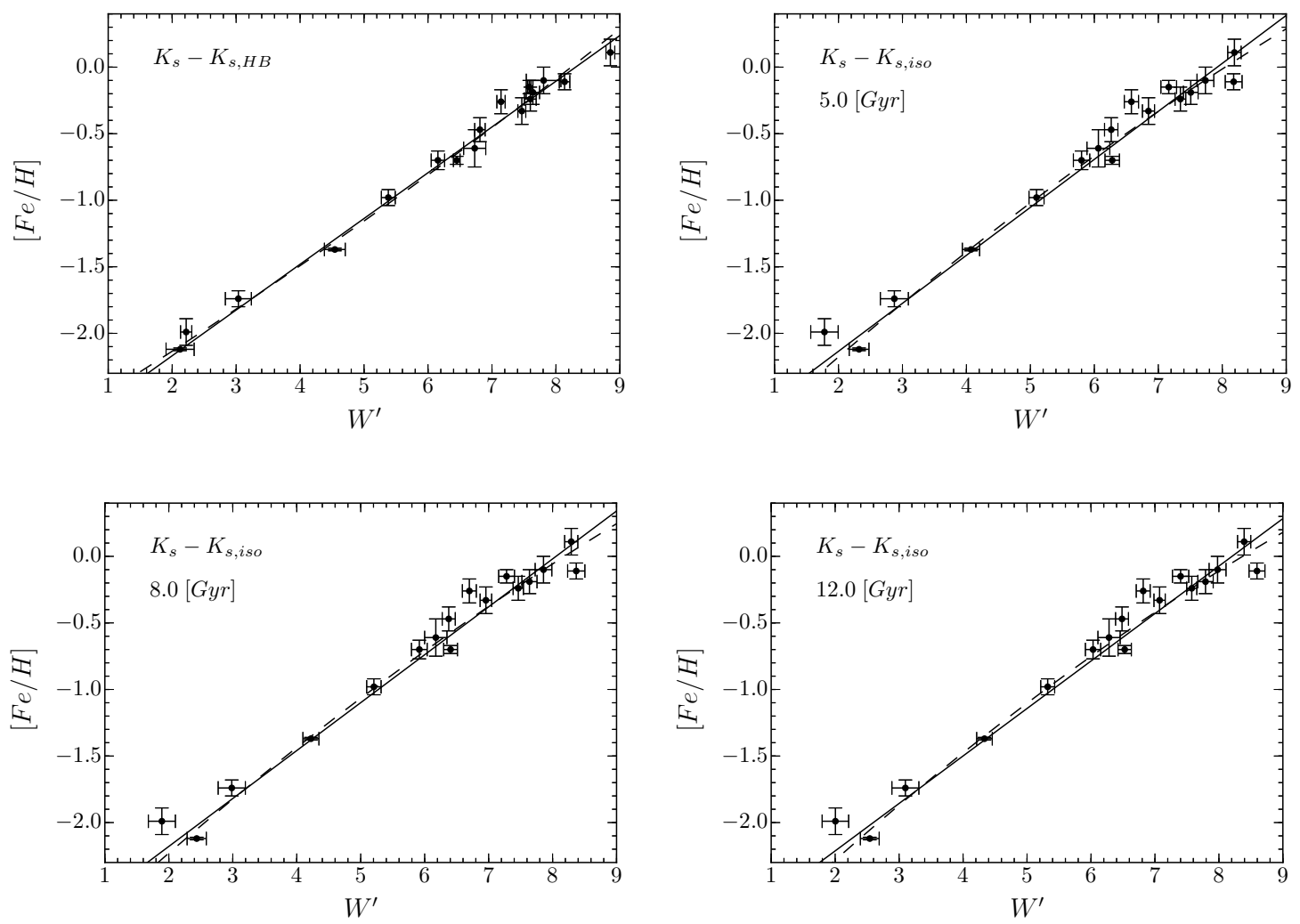

Fig. 2.5.- Reduced equivalent width-metallicity relations, and the linear (solid line) and quadratic (dashed line) best-fits. For the upper-left panel, $W^{\prime}$ values were calculated from $K_{s}-K_{s, H B}$, while values for $W^{\prime}$ in the upper-right and bottom panels come from $M\left(K_{s, i s o}\right)$ for 5.0, 8.0 and 12.0 Gyr isochrones - derived from $\left(J-K_{s}\right)_{o}$ colors. The isochrone-based method (described in Section 2.3.2) also follows a linear relation in this plane, but the scatter around the fit is larger. Note that the three relations from isochrones have a quite similar appearance, despite different isochrone age used to derive $W^{\prime}$ in each case. 

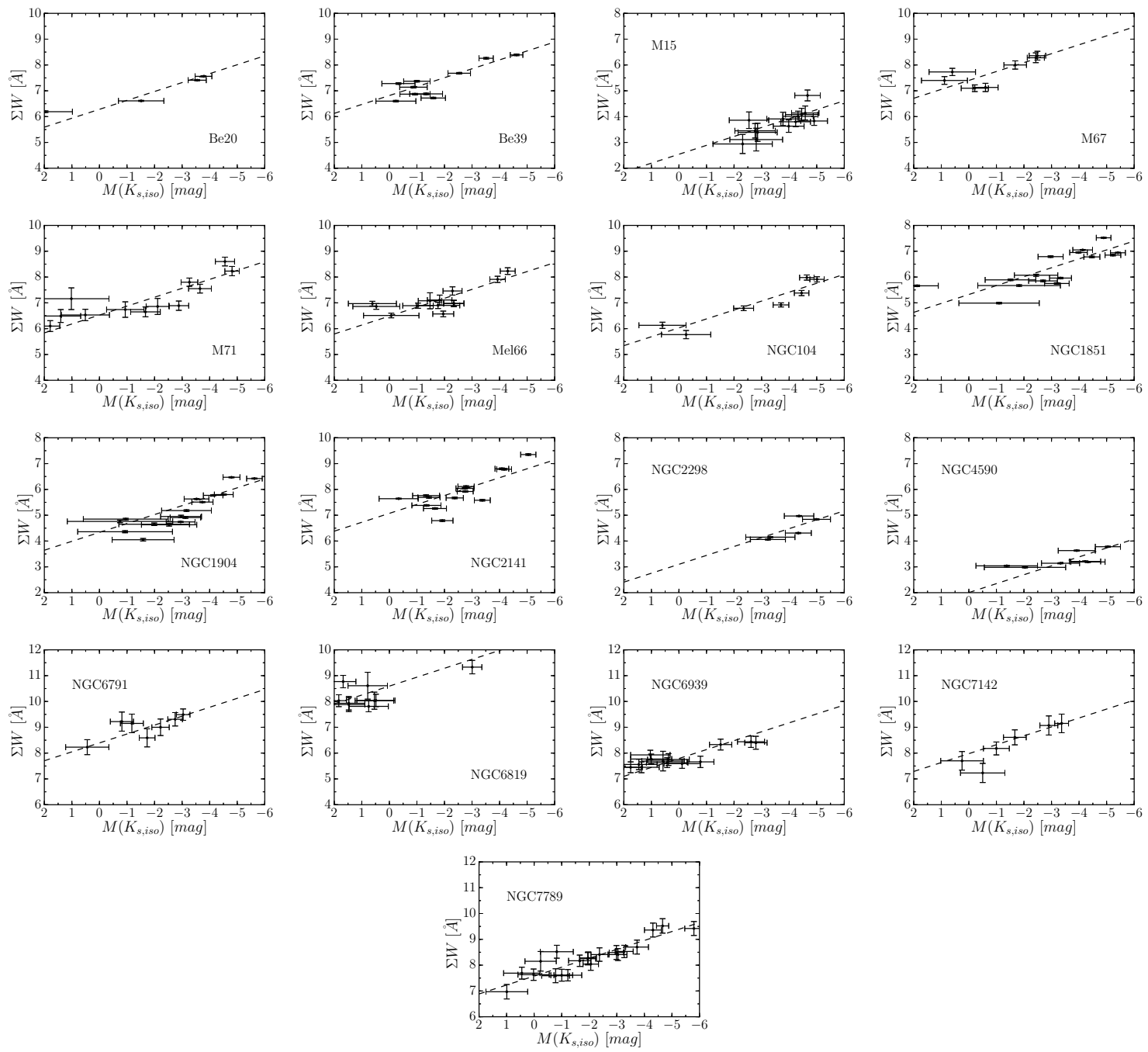

Fig. 2.6. - The calibration clusters in the $\left(M\left(K_{s, i s o}\right), \Sigma W\right)$ plane, calculated from 8 Gyr isochrones. The dashed line shows the best-fit results. 

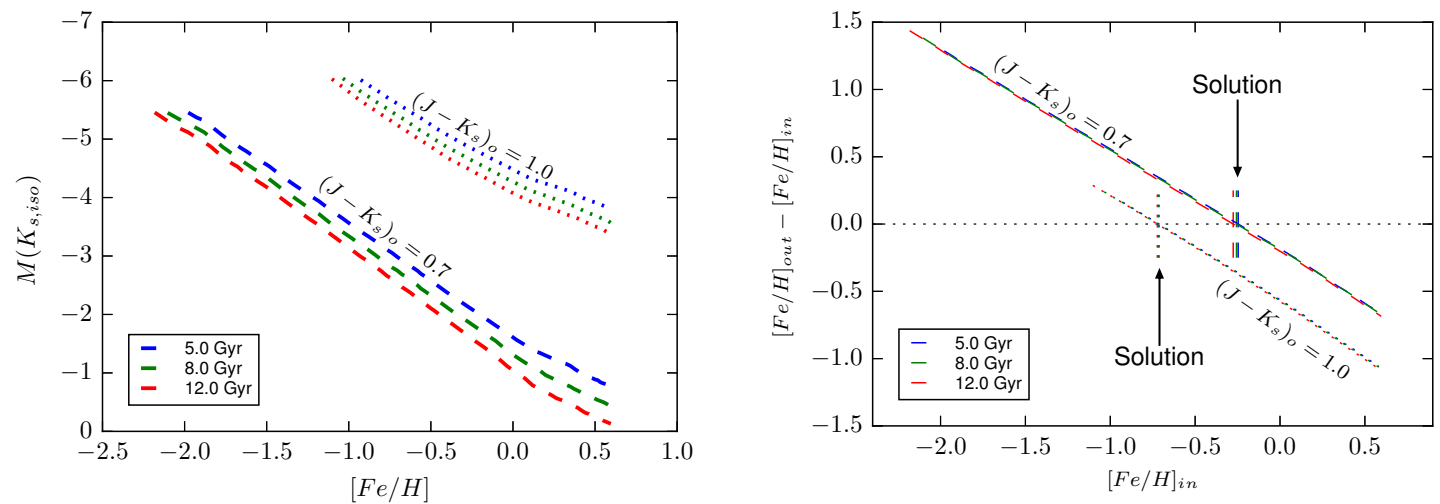

Fig. 2.7. - Left: Isochrone metallicity versus $M\left(K_{s, i s o}\right)$, for 5,8 and 12 Gyr. We show relations for $\left(J-K_{s}\right)_{o}=0.7$ and 1.0. Right: Examples of the metallicity measurement based on isochrones, for stars with $\left(J-K_{s}\right)_{o}=0.7$ and 1.0 , and $\Sigma W=8.0 \AA$. The tracks correspond to the results of evaluating the $M\left(K_{s, i s o}\right)$ values on the left panel with the CaT relations derived in Section 2.3.2. The difference between the isochronebased metallicity (from the calibration) and the actual isochrone metallicity is a monotonically-decreasing function of the latter. The solution is found where input and output metallicities are equal. Note how even an age difference of 7 Gyr between two of the isochrone sets produces basically identical results. 

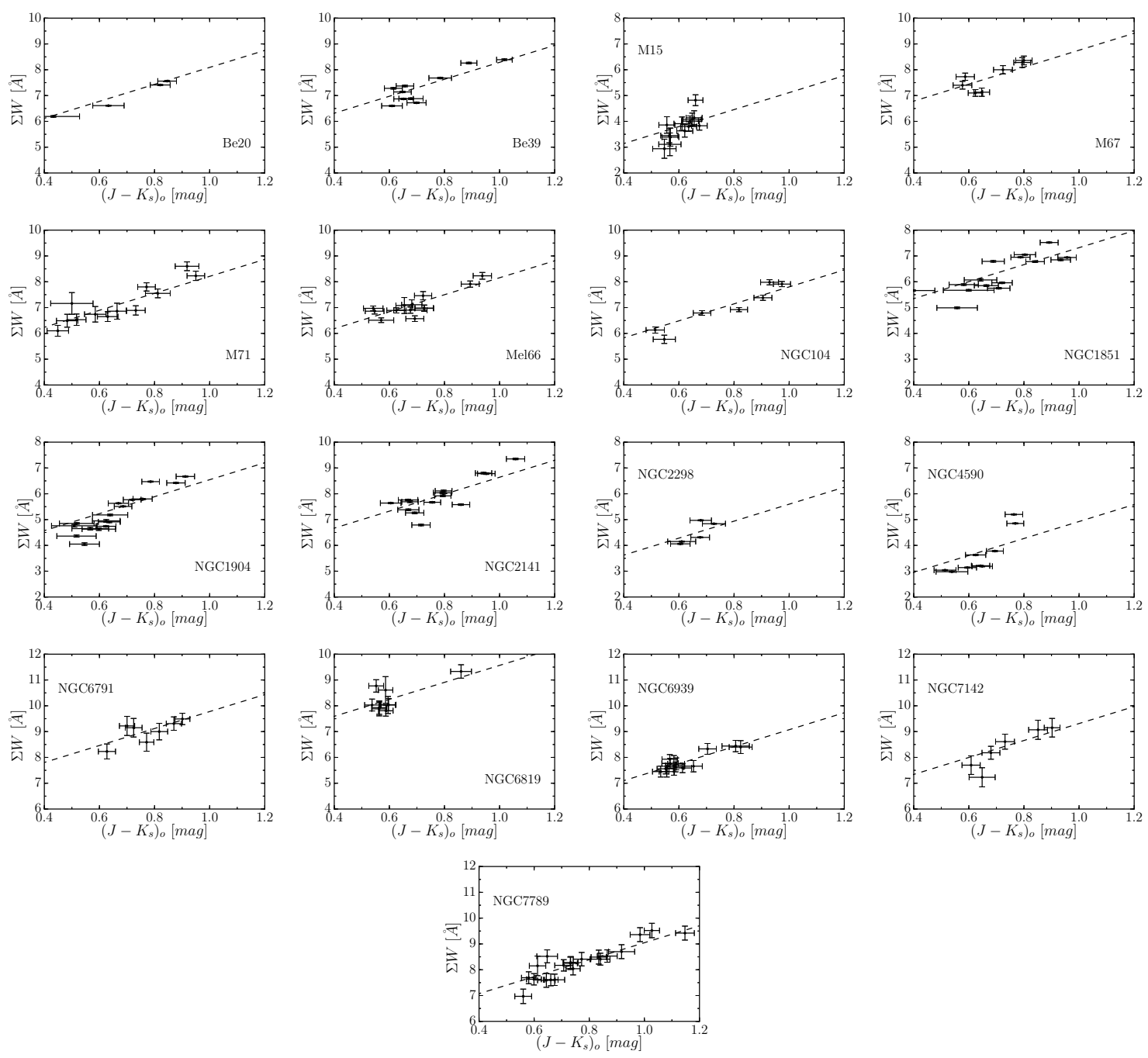

Fig. 2.8.- Best-fit results for the calibration clusters in the $\left(\Sigma W,\left(J-K_{s}\right)_{o}\right)$ plane. 

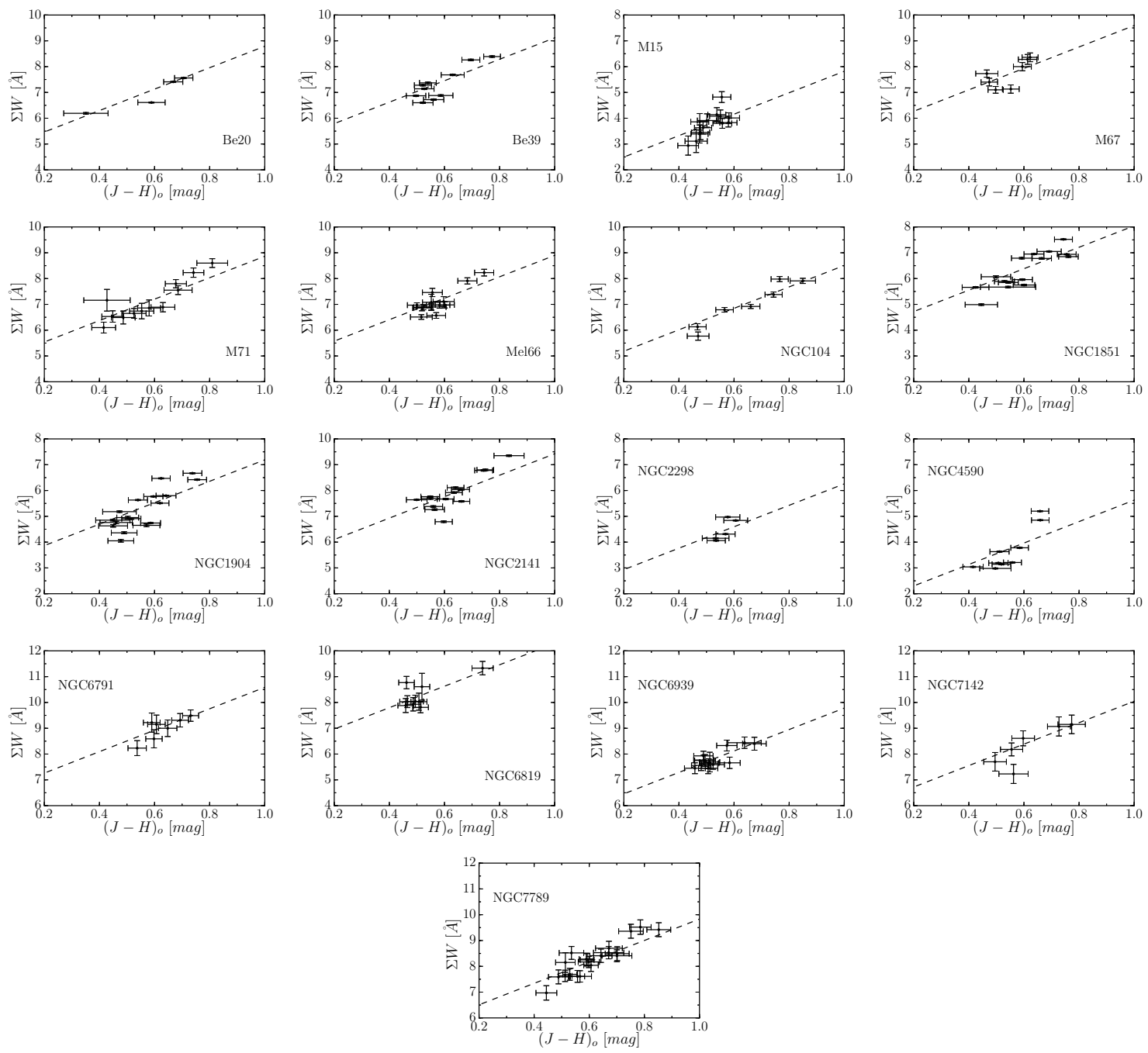

Fig. 2.9.- Best-fit results for the calibration clusters in the $\left(\Sigma W,(J-H)_{o}\right)$ plane. 

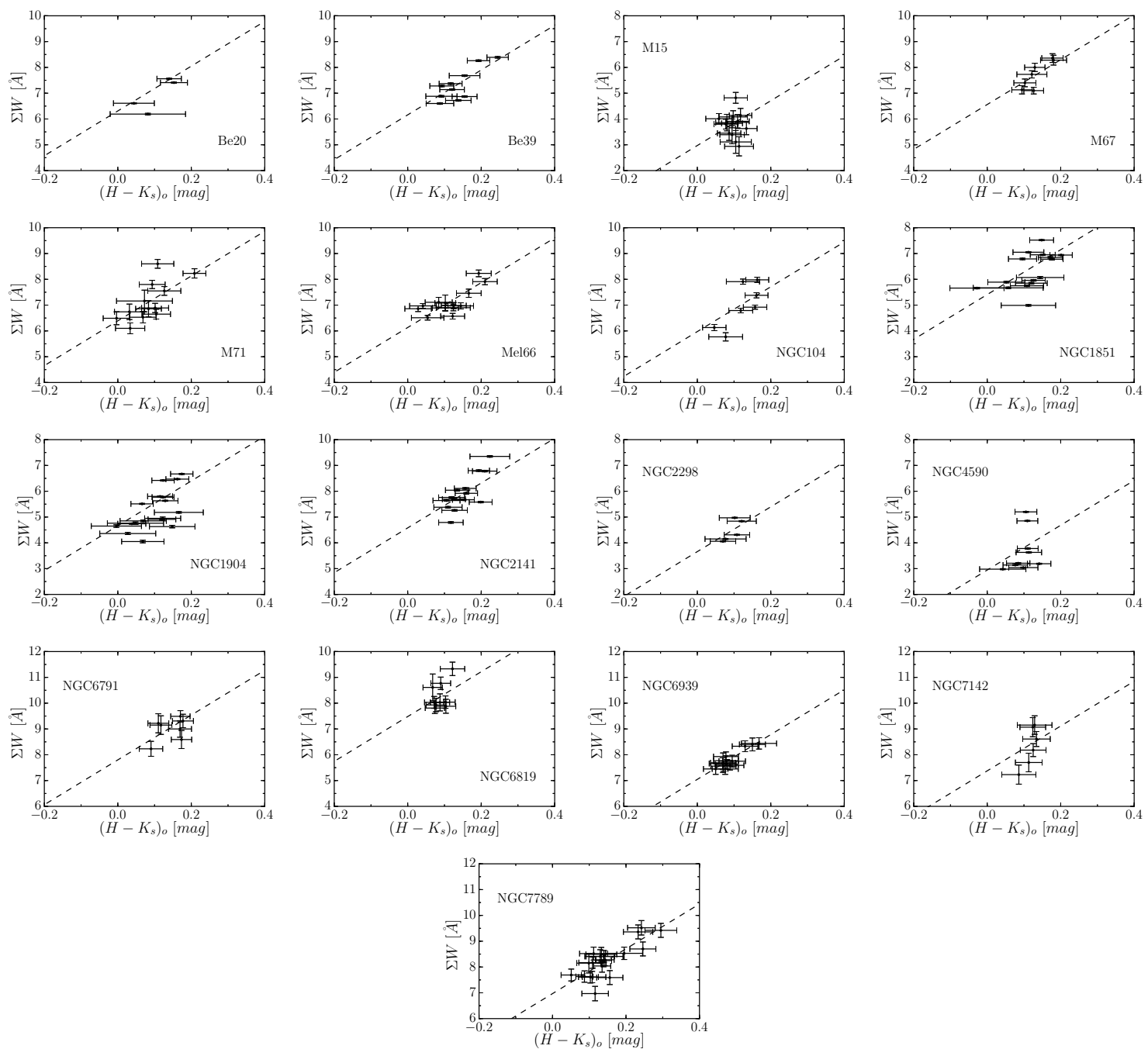

Fig. 2.10.- Best-fit results of the calibration clusters in the $\left(\Sigma W,\left(H-K_{s}\right)_{o}\right)$ plane. 

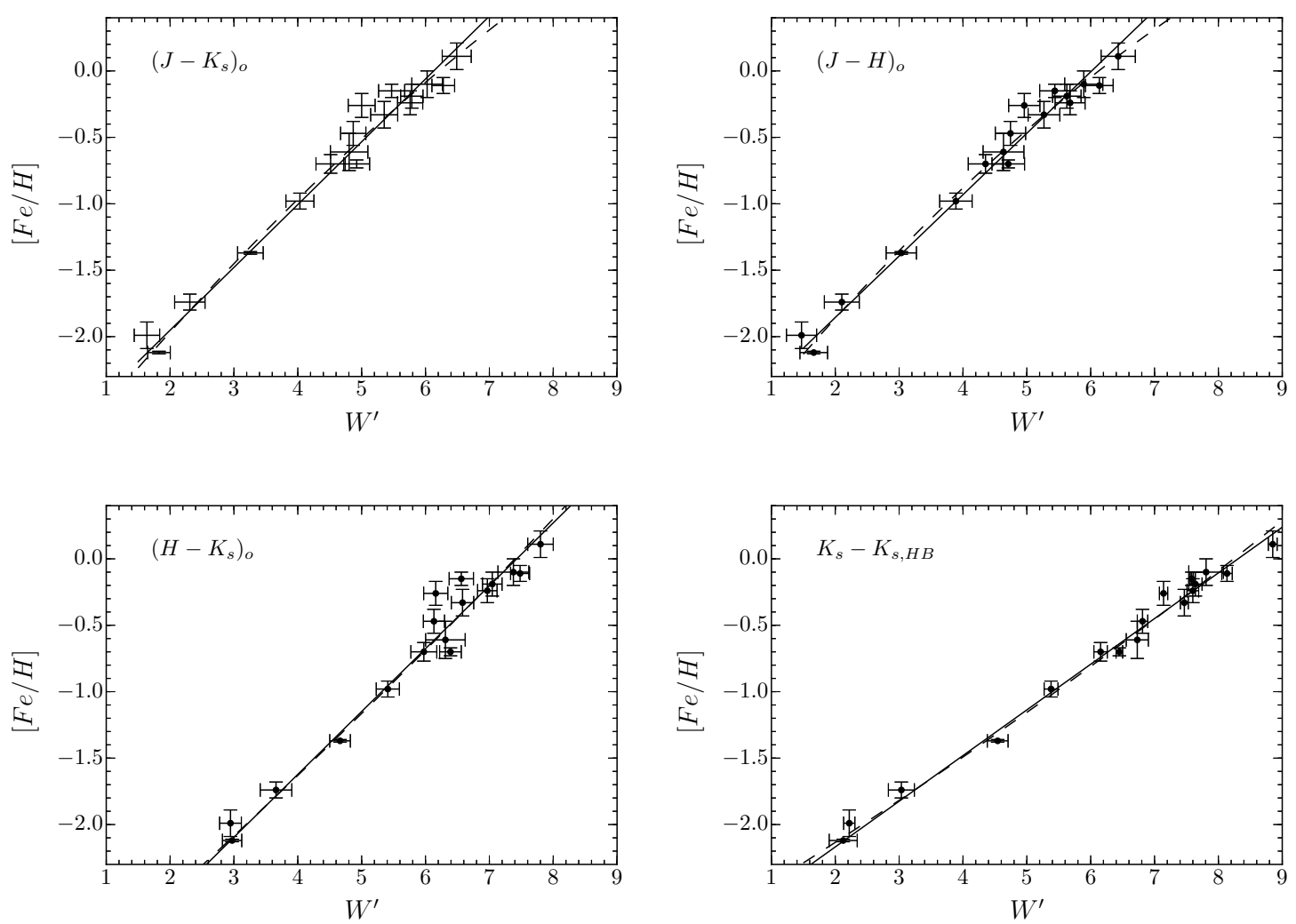

Fig. 2.11. - Reduced equivalent width-metallicity relation based on the three color combinations of the 2MASS bands. The solid (dashed) line is the best linear (quadratic) fit. It is clear that the data follow linear relations in these planes, as the linear and quadratic relations are quite similar. We have included the plot of the HB-based calibration for comparison purposes (lower right panel). 

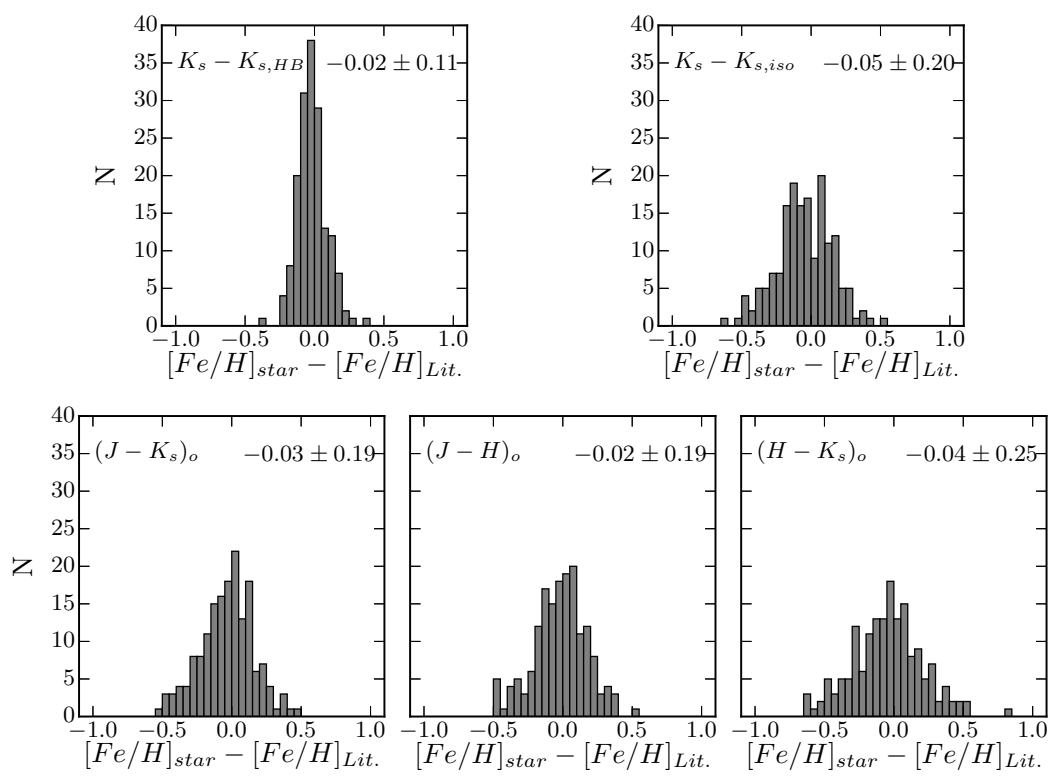

(a) Difference between CaT metallicity and literature values for individual stars in the whole calibration sample. The numbers on each panel indicate the mean and standard deviation of the data.
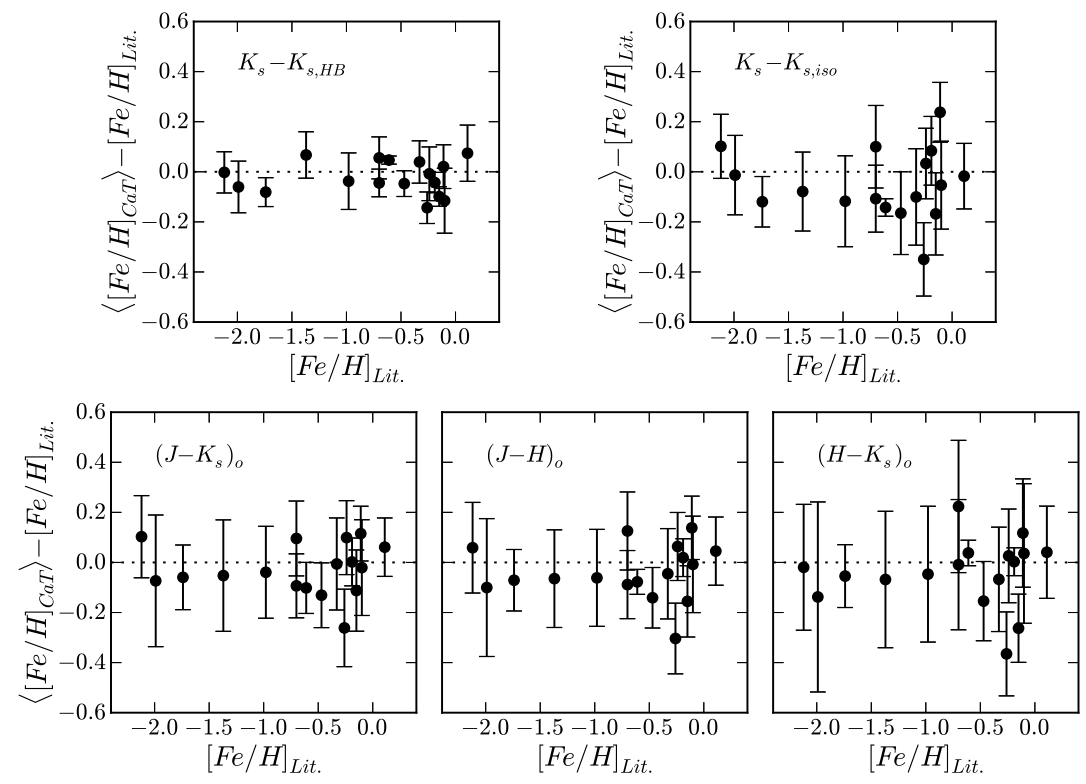

(b) Weighted mean metallicity for clusters from CaT calibrations compared against literature values. Error bars represent the standard deviation of the weighted mean.

Fig. 2.12.- Comparison of the metallicity scatter between the five CaT calibrations discussed in this chapter. We use the literature values as the reference metallicity values. 

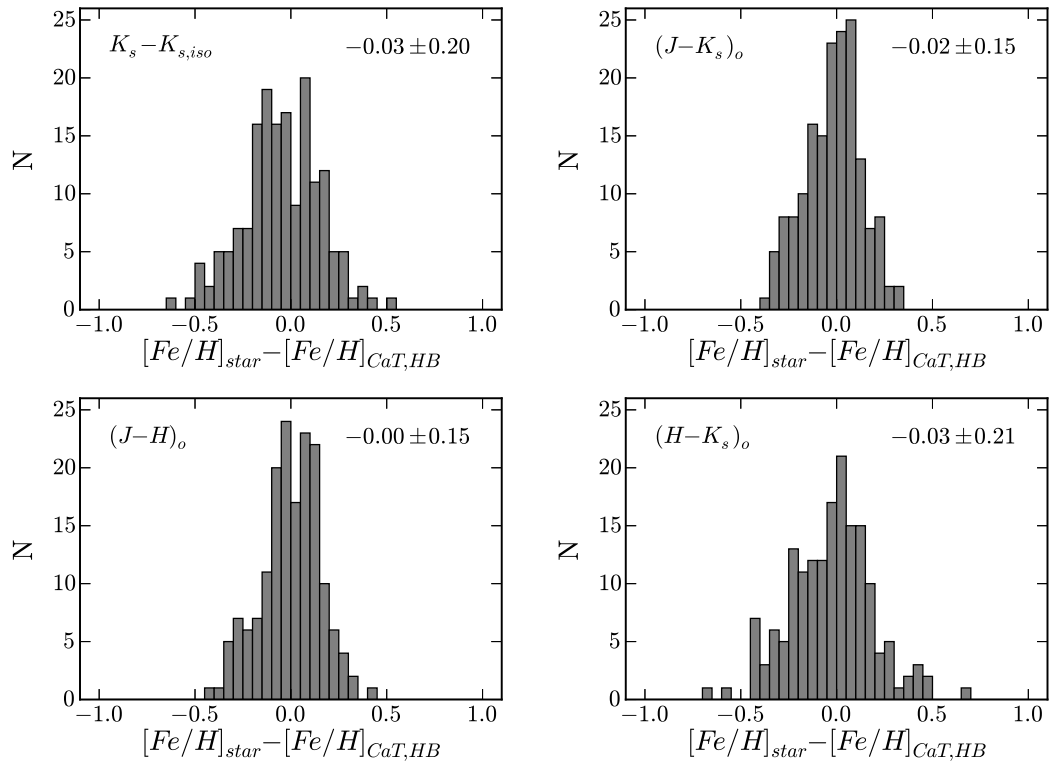

(a) Difference between metallicities from the CaT calibrations that we propose, for individual stars in the whole calibration sample, compared against results from the HB-based CaT calibration. The numbers on each panel indicate the mean and standard deviation of the histograms.

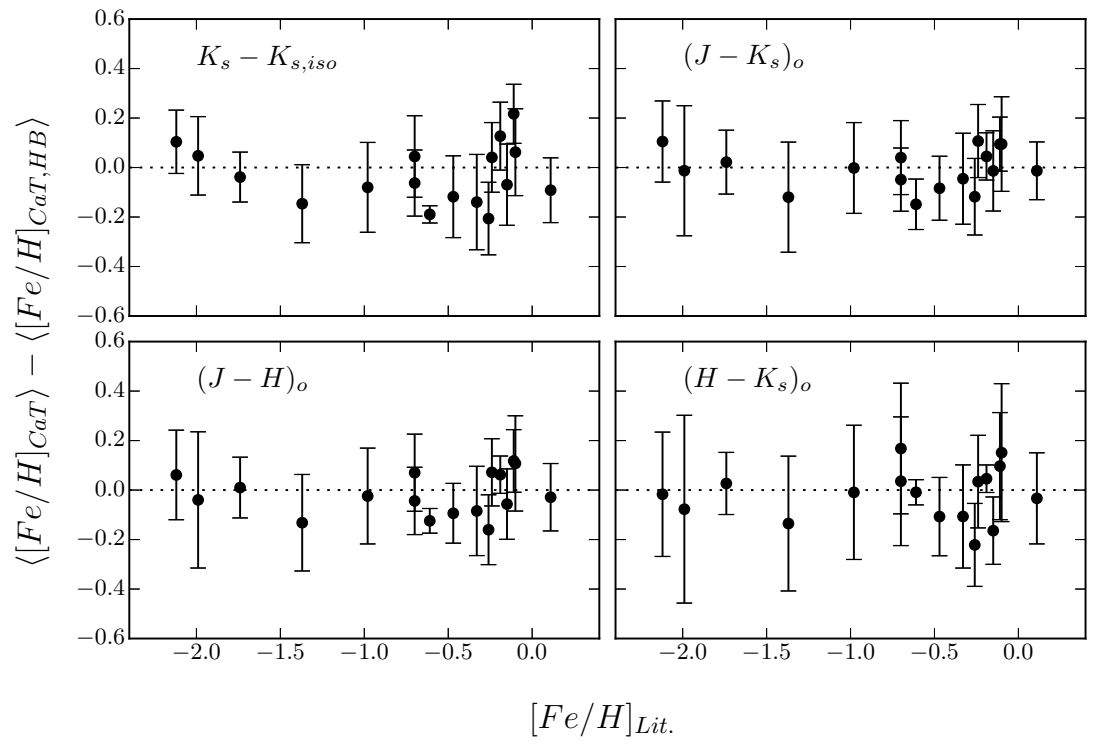

(b) Weighted mean metallicity for clusters, for the CaT calibrations that we propose, compared against results from the HB-based calibration. Errorbars represent the standard deviation of the weighted mean for each cluster.

Fig. 2.13. - Estimation of the actual scatter of metallicity values for the proposed CaT calibrations. We compare our metallicity results against values from the HBbased CaT for the sample of calibration clusters. This comparison removes the intrinsic metallicity scatter of the HB-based CaT calibration from the analysis. 

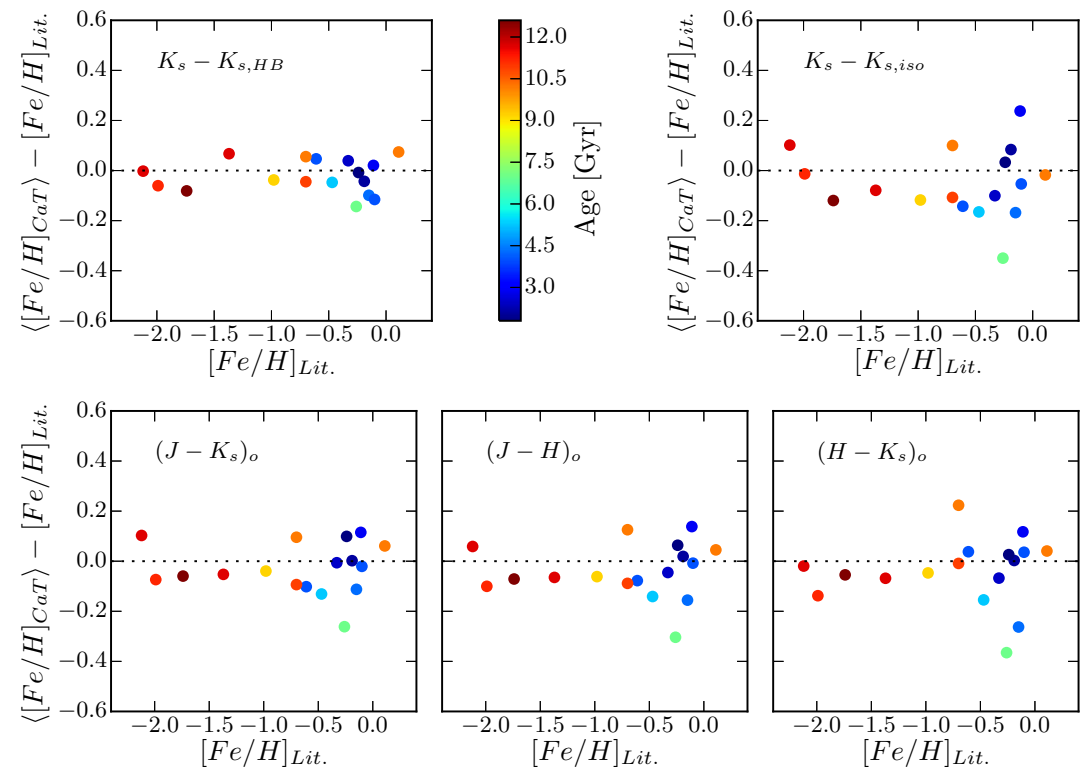

(a) Differences compared against literature metallicities (same as figure $2.12 \mathrm{~b}$ ), color-coded by cluster age.

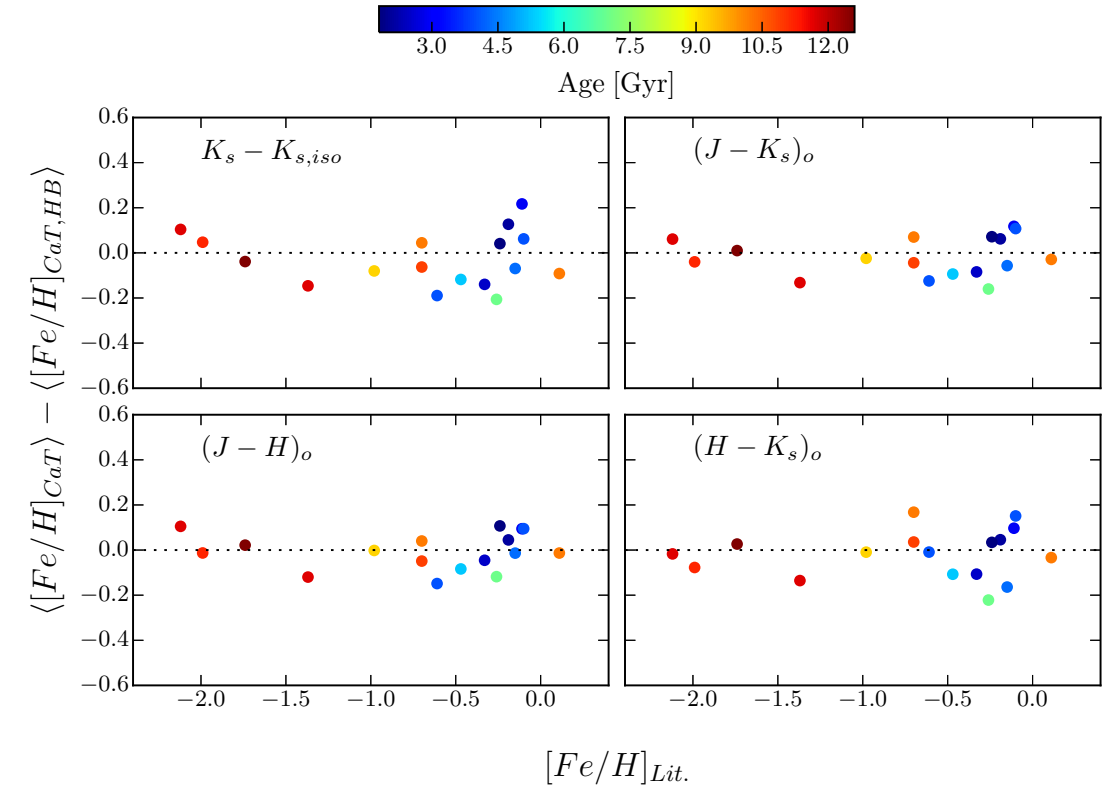

(b) Differences compared against HB-based CaT metallicities (same as figure $2.13 \mathrm{~b}$ ), color-coded by cluster age.

Fig. 2.14. - Weighted mean metallicities for the calibration clusters, color-coded by cluster age. 

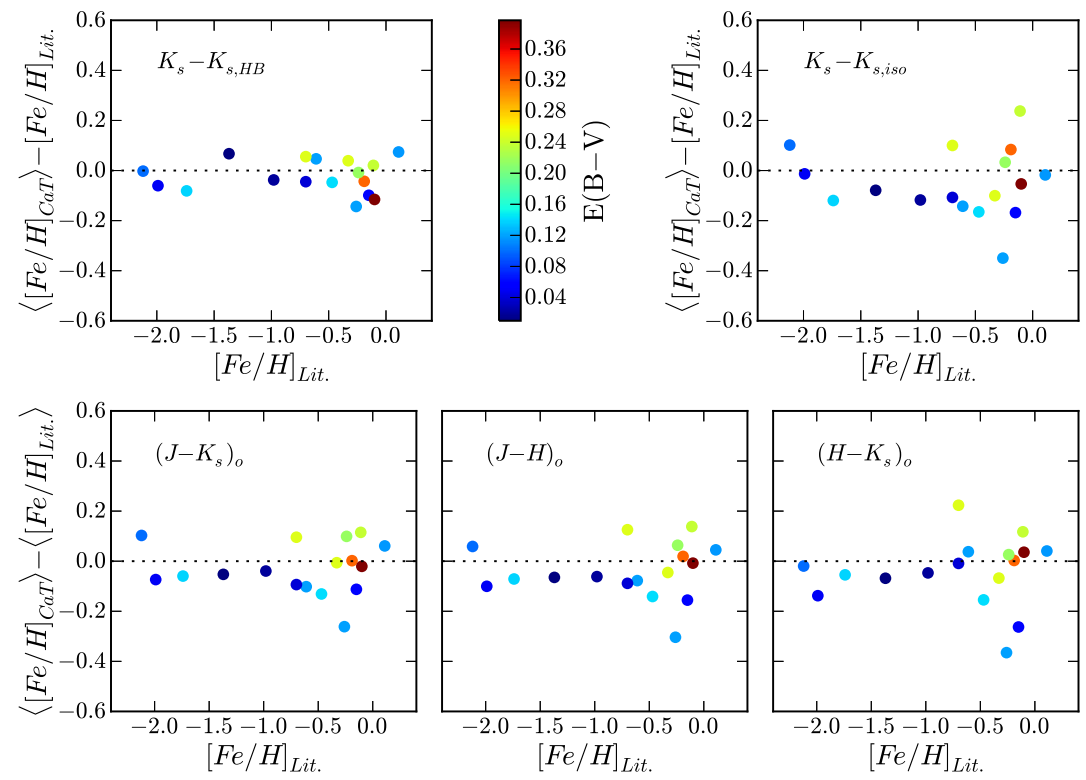

(a) Differences compared against literature metallicities (same as figure $2.12 \mathrm{~b})$, color-coded by cluster $E(B-V)$.

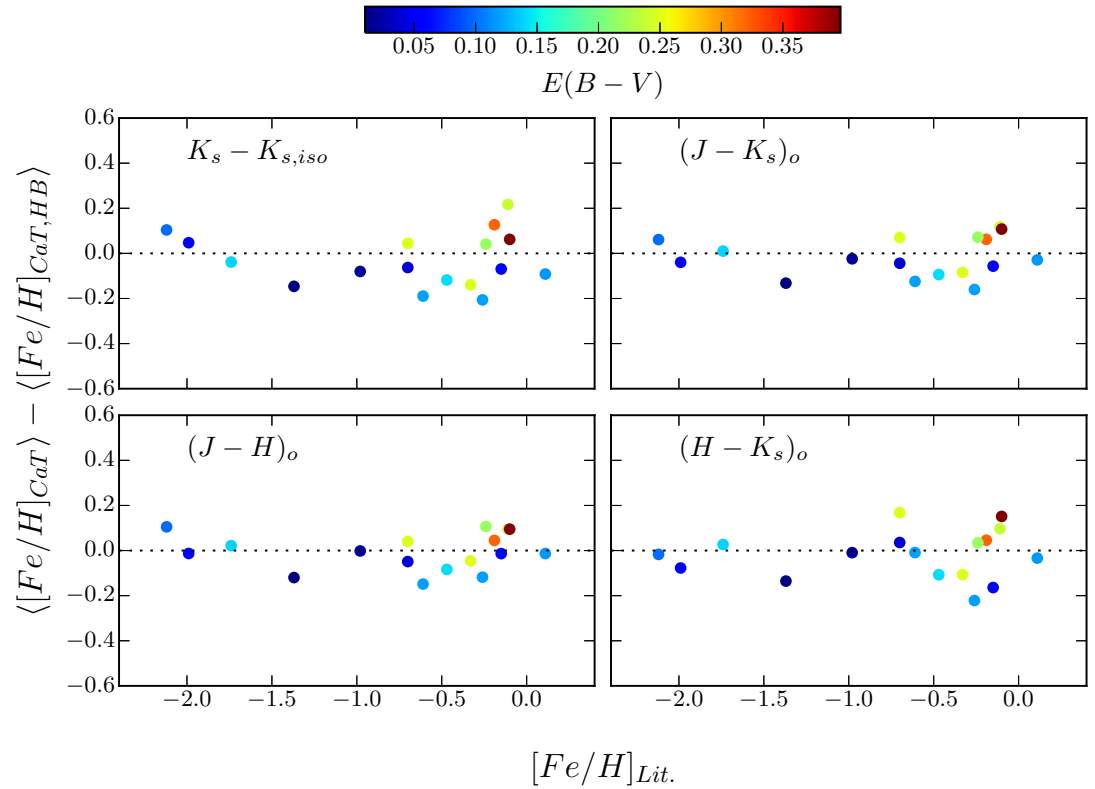

(b) Differences compared against HB-based CaT metallicities (same as figure $2.13 \mathrm{~b})$, color-coded by cluster $E(B-V)$.

Fig. 2.15.- Weighted mean metallicities for the calibration clusters, color-coded by cluster $E(B-V)$. 


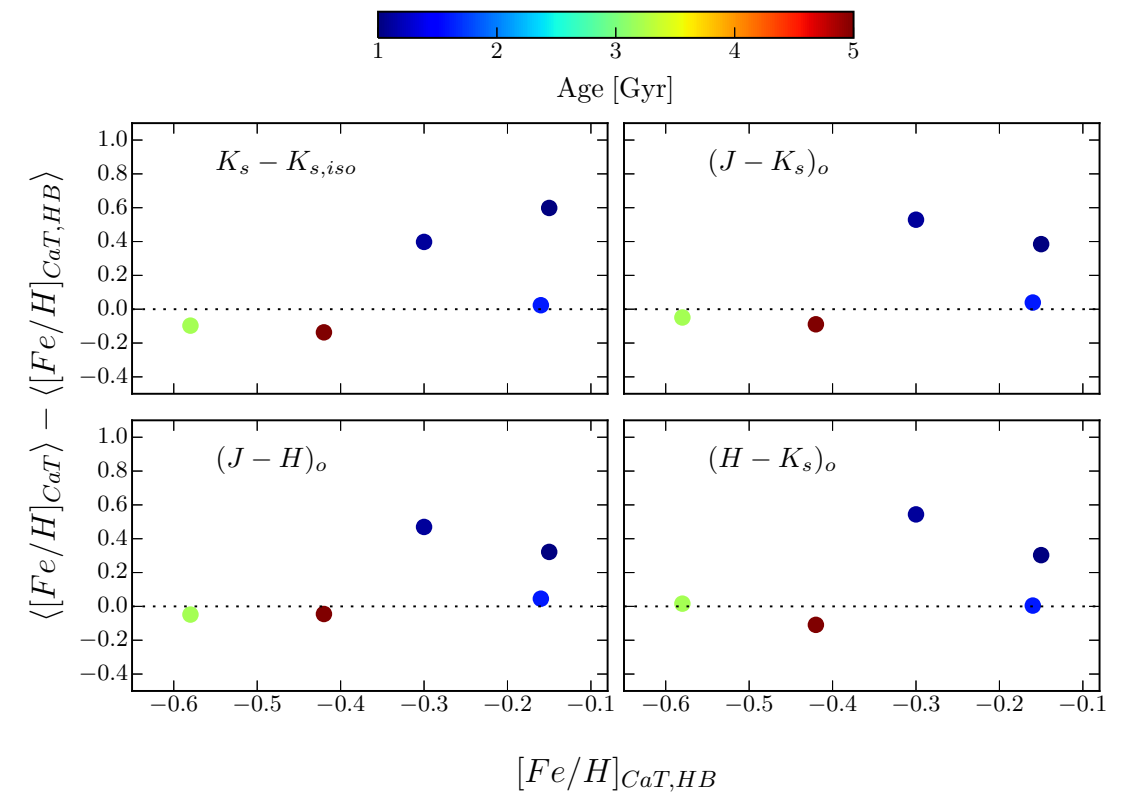

(a) Metallicity differences color-coded by cluster age

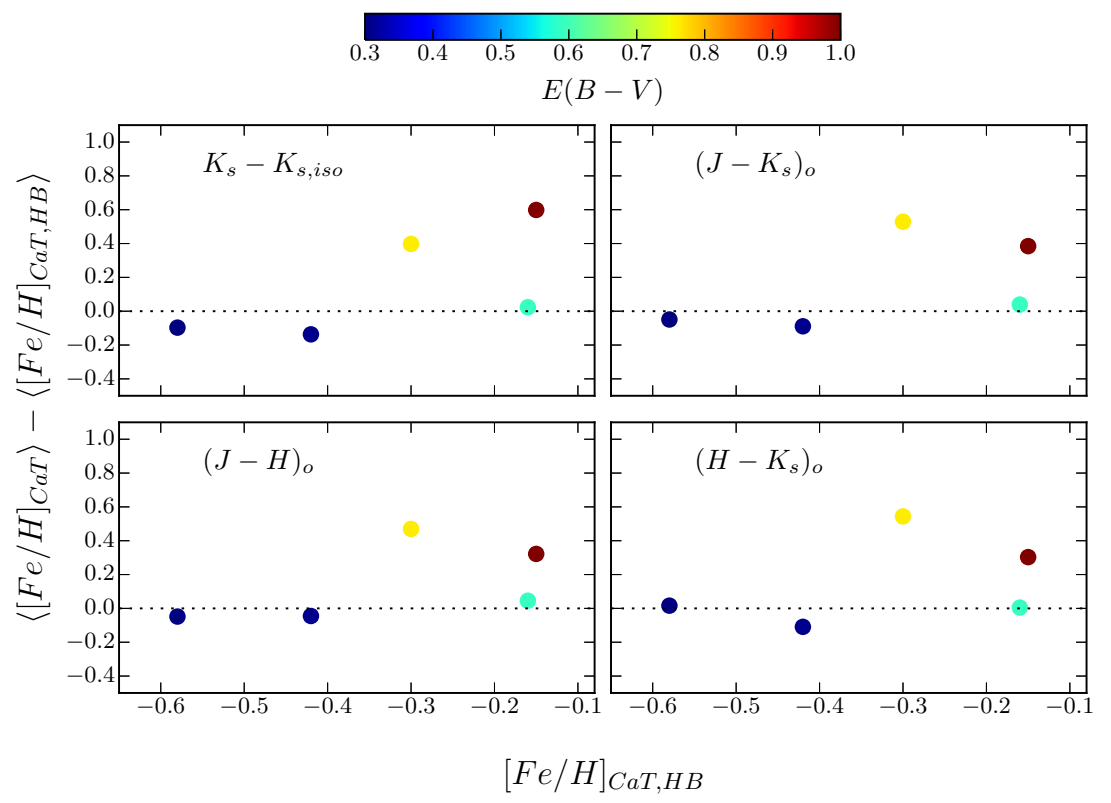

(b) Metallicity differences color-coded by cluster E(B-V)

Fig. 2.16. - Comparison of weighted-average metallicity per cluster, for the proposed CaT calibrations against HB-based CaT metallicity. These clusters were not part of the calibration process, then this set of clusters provide an opportunity to test the new calibrations. This comparison removes the intrinsic scatter from the HB-based CaT metallicity from the analysis. 


\section{Chapter 3}

\section{Tracing the Tidal Tails of the Sagittarius Dwarf using M Giant Stars}

\subsection{Introduction}

The Sagittarius dwarf galaxy (Sgr hereafter) represents the best example known to date of ongoing tidal disruption of a galactic satellite by the Milky Way Galaxy. Despite this fact, the core of Sgr remained hidden behind the Galactic Center, swamped by the many foreground Milky Way stars, until Ibata et al. (1994) discovered it in a spectroscopic survey of the Galactic bulge. They immediately noticed Sgr's elongated shape towards the Galactic plane, and inferred that it was under active tidal disruption. Soon after the discovery of Sgr, it was recognized that its current location along its orbit provides a unique example to study the effects of strong tides during disruption, through N-body simulations (Johnston et al. 1995). Early N-body simulations predicted that debris from Sgr should be present as coherent tails in the Galactic 
halo, and soon evidence for extended debris was found using a variety of techniques used to detect its tidal structures. For example, Ibata \& Lewis (1998) used carbon stars to trace around $100^{\circ}$ of the leading arm.

Majewski et al. (2003, M03, hereafter) revealed the first all-sky view of the Sgr system — whose tidal tails extends over $2 \pi$ on the sky. M03 showed that an appropriate color-color selection from 2MASS (Skrutskie et al. 2006) can effectively be used to discriminate M-giants, which are a good tracer of the relatively metal-rich tidal tails of the dwarf. Subsequent studies based on M-giants selected from 2MASS have provided a wealth of information about the Sgr system. For instance, Majewski et al. (2004) measured radial velocities in a spectroscopic sample of bright M-giants along the Sgr tails. In addition, M-giants have been used as tracers to calibrate models of the MW potential, characterize the Sgr progenitor and the tidal debris expelled as part of the (ongoing) disruption (e.g., Law et al. 2004, 2005; Johnston et al. 2005; Law et al. 2009; Law \& Majewski 2010).

Other extensive, deep photometric optical surveys, such as the Sloan Digital Sky Survey (SDSS) and the Panoramic Survey Telescope and Rapid Response System 1 (Pan-STARRS1), have proven useful for detection of Sgr debris and brought new insights about the stream through different stellar tracers. For example, Belokurov et al. (2006) measured the density of Main Sequence Turn Off in the SDSS and discovered that the Sgr leading arm is bifurcated into two parallel streams. Later, Koposov et al. (2012) used the same methodology of Belokurov et al. (2006) for SDSS data around the South Galactic Cap to find that the trailing arm was also bifurcated.However, no model to date has been capable of explaining the bifurcated nature of Sgr arms. For instance, Peñarrubia et al. (2010) used N-body simulations to show that bifurcated tails can be the result of the accretion of a rotating disk 
satellite. On the other hand, Frinchaboy et al. (2012) measured radial velocities of 1,310 Sgr core stars from medium resolution spectra and found no evidence of significant rotation at the level required to explain the bifurcated arms.

Following the Majewski et al. (2004) publication, many more spectra of 2MASSselected M-giants were pursued by Majewski + students. These data have never been formally published, although some of the data were used in Law et al. (2005); Law \& Majewski (2010) to constrain N-body models of the stream. This thesis chapter represents the most thorough consolidation and analysis of these various M-giant spectra to date.

\subsection{Data Acquisition, Reduction and Measurements}

\subsubsection{Spectroscopic Target Selection}

In addition to the information provided by the spatial location and stellar density of Sgr tidal tails in the Galaxy, a wealth of information about the stream progenitor and its tidal disruption can be learned from the kinematics and chemistry of the debris. Furthermore, stream dynamics can probe the Galactic dark matter halo, as well as the details of tidal disruption processes (e.g., Law \& Majewski 2010). Therefore, spectroscopic studies of stars in Sgr system represent a great opportunity to learn about our Galaxy, its mass distribution and its evolution and growth through minor mergers. For these reasons, we extend the spectroscopic search of Sgr M-giants of Majewski et al. (2004), using target candidates from 2MASS. Our search was initially motivated by the possibility of extended tails that were apparent in Figure 9 of M03 (Fig. 3.1).

M-giants comprise one of the best types of stars to trace Sgr debris for several 


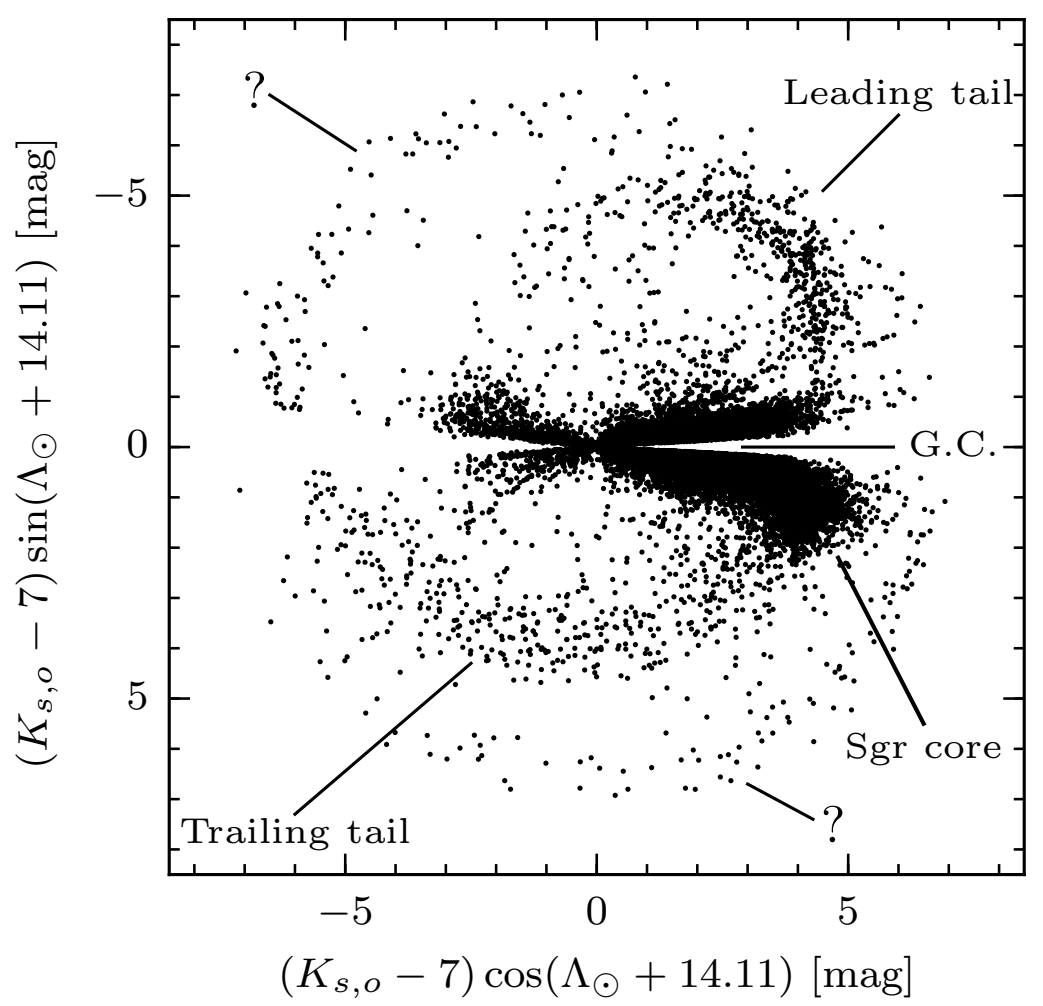

Fig. 3.1. - The original view of Sgr revealed by Majewski et al. (2004) using M-giants selected from 2MASS. The question marks indicate the two ring-shaped structures visible in M03 that motivated this work. Figure based on the paper of Majewski et al. (2004).

reasons. First, these stars are intrinsically bright objects. Second, the presence of relatively metal rich populations in Sgr means that it has a very large M-giant population, while these stars are not common in the outer Galaxy. M03 showed that it is possible to select M-giants using a simple color-color cut in the all-sky 2MASS data. Third, the relatively low effective temperature of M-giants make them bright in the near IR. Fourth, the near IR region around $\lambda 8,500 \AA$ is dominated by the Ca II triplet absorption lines (Chapter 2). Therefore, the Calcium triplet feature in M-giants is an attractive target for spectrographs of moderate resolution to measure 
radial velocities.

We start the search by selecting M-giant spectroscopic candidates from 2MASS, following the prescription from $\mathrm{M}^{1} 3^{1}$. The method utilizes the giant/dwarf differentiation in $\left(\left(J-K_{S}\right)_{o}\right.$ vs. $\left.(J-H)_{o}\right)$ space that is observed in stars redder than $(J-K s)_{o} \gtrsim 0.85$. The separation is caused mainly by absorption from $H^{-}$and $H_{2}^{-}$ at 1.6 microns in the $\mathrm{H}$ band (Lee 1970). We dereddened the 2MASS photometry using the $E(B-V)$ maps from Schlegel et al. (1998), and a total-to-selective extinction ratio, $R_{V}=A_{V} / E(B-V)$ equal to 3.1. To convert the $V$-band extinction into 2MASS bands, we used the ratio $A_{V} / A_{K} \sim 8.8$ from Cardelli et al. (1989) and the $A_{J} / A_{K}$ and $A_{H} / A_{K}$ ratios from Indebetouw et al. (2005), previously introduced in equations 2.3, 2.4 and 2.5.

After identifying M-giants candidates, we select the ones that most likely belong to Sgr tidal debris. To do so, we keep stars that lie close to the equator of Sgr coordinatesystem defined by M03. This spherical coordinate system places the equator along the great circle traced out by 2 MASS M-giants in the Sgr stream ${ }^{2}$. We are specially interested in the faint end of the selected group.

\subsubsection{Spectroscopic Data Acquisition and Reduction}

Spectra of the selected candidates, from the Northern and Southern hemispheres, were collected over a total of 10 observing runs carried out between years 2004 and 2014. The targets span a broad range of $K_{s}$ magnitudes, and required telescopes of different apertures. Figure 3.2 illustrates the stars that were targeted in this exhaustive campaign.

\footnotetext{
${ }^{1}$ As we shall see, the catalog used by M03 was a proprietary, pre-release version of 2MASS that was subsequently revised.

${ }^{2}$ The coordinate system longitude $\Lambda_{\text {odot }}$ has its zero point at Sgr core, and increases along Sgr trailing arm, while latittude $B_{\text {odot }}$ increases towards the south celestial pole.
} 


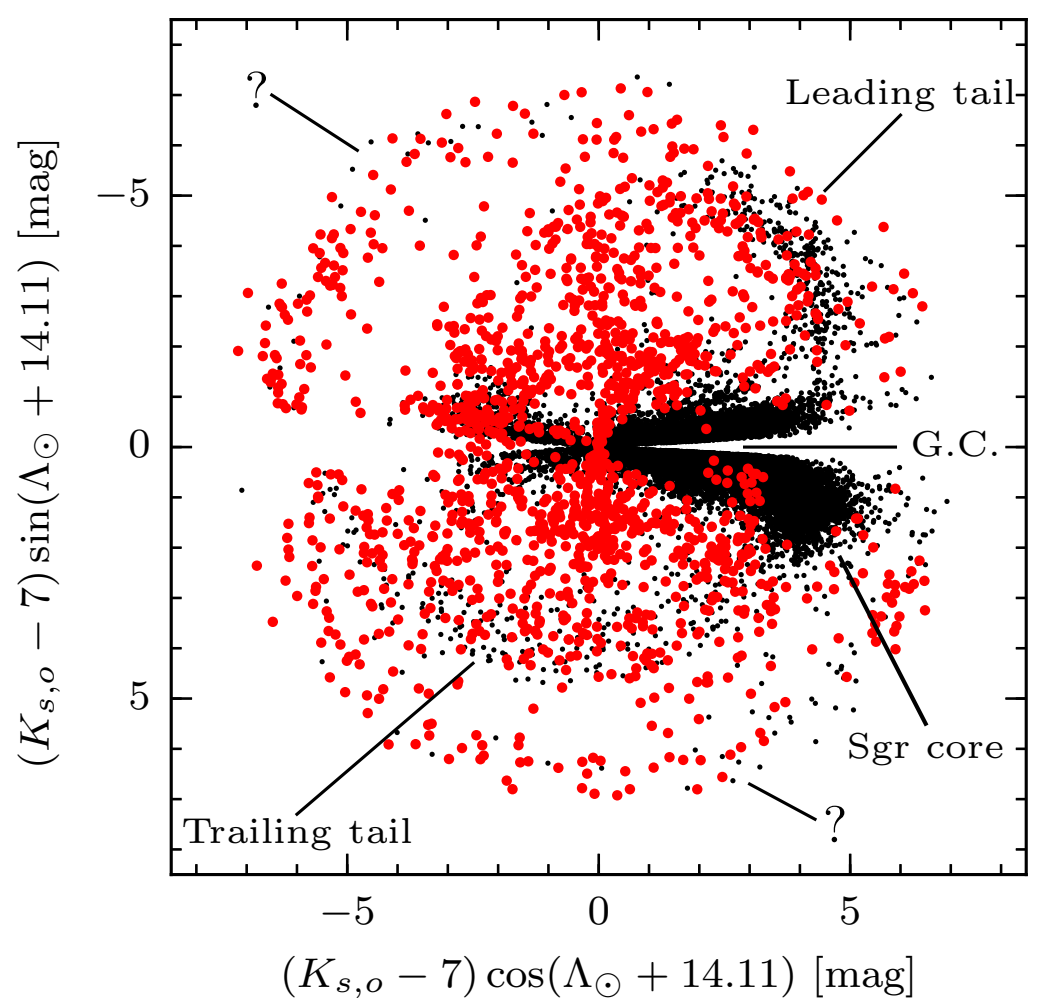

Fig. 3.2.- Same as Figure 3.1, but with the stars targeted for spectroscopy by our group shown in red dots. This figure includes the sample from Majewski et al. (2004)

Details about each run, such as telescope, instrument, and UT dates, are summarized in Table 3.1. All spectra were obtained using long-slit spectrographs, and with the optics configured to the spectral region of the Ca II triplet. In each run, we took sets of bias, flat-field and comparison-lamp calibration frames, as well as spectra of giant and dwarf radial velocity standards whose colors span the range of colors found in our sample of science targets.

We followed the standard procedure in $\mathrm{IRAF}^{3}$ for the reduction of CCD data from

\footnotetext{
${ }^{3}$ IRAF is distributed by the National Optical Astronomy Observatory, which is operated by the Association of Universities for Research in Astronomy (AURA) under cooperative agreement with the National Science Foundation.
} 
each run, with the ccdproc package. This procedure includes overscan and bias subtraction, and flat-field correction of the 2D images. Next, we use the doslit task in IRAF to trace, extract, and wavelength-calibrate these spectra using a reference spectrum. Calibration spectra for the last step were obtained from internal comparison lamps available in each spectrograph ( $\mathrm{He}, \mathrm{Ne}$ and/or $\mathrm{Ar}$ ), with exposures taken immediately before or after each science or RV-standard spectrum. These calibration frames are critical for correcting instrument flexure effects in the wavelength calibration. We extracted these comparison spectra using the same $2 \mathrm{D}$ trace as for the stellar spectra, identified the lamp lines, and then, fitted a low-order polynomial to transform from pixels into wavelengths (in air).

\subsubsection{Radial Velocities Measurement}

The radial velocities $(\mathrm{RVs})$ for all the stellar spectra were measured via the crosscorrelation methodology of Tonry \& Davis (1979), which is implemented in the IRAF package FXCOR. We followed the same procedure for each observing run, which consists of: choosing an appropriate RV-standard template spectrum from the set of RVstandards observed on the run, measuring RVs for the spectra of objects with respect to the RV-template, estimating RV errors, correcting the object RVs for any possible RV offset of the template, and converting the observed velocities into heliocentric frame. These steps are described in detail in the following subsections.

As mentioned earlier, for each observing run we obtained spectra with high signalto-noise ratio (SNR) of several RV standard stars. These have a threefold purpose: First, they serve as the cross-correlation template; second, they can be used to evaluate any RV offset in the template spectrum chosen from all the spectra; and third, they provide a means to assess the random error of the RV measured for each spec- 
trum.

\section{Standard Stars and Selection of Cross-Correlation Templates}

The main requirement for choosing an RV template spectrum is that it matches the spectral features of the objects, which will result in a stronger cross-correlation peak, and therefore smaller random errors in the RVs of our targets. We usually have several template candidates within each run, since all of our standard stars present strong Ca triplet lines for the cross-correlation. Initially, we cross-correlated each object spectrum against all of these standard spectra and obtained an average and standard deviation of these measurements. However, the scatter in these RV results, for a single object spectrum measured against multiple templates, mainly reflects the literature RV uncertainty within the set of templates, but not the error in the RV measured for the object spectrum. Because of this, it is more convenient to select a single high-quality template, and estimate more realistic RV errors later using a different approach.

That said, we have to determine which one is the best template available in a run. To answer this, we began by cross-correlating each RV-standard spectrum against the remaining N-1 template candidates, and obtained N-1 velocities in the heliocentric frame. Beforehand, the input spectra for FXCOR are trimmed, and only the regions

within $8450<\lambda<8850$ Åkept. Moreover, we set the task to apply a ramp-filter in Fourier space, which suppresses the very low and very high frequencies, and do a background normalization by fitting a low order polynomial to the continuum. In addition, we masked out both the object and template data, except for the strong Ca II triplet lines, because the other spectral regions with weak lines basically introduce noise into the cross-correlation. The actual spectral regions that are cross-correlated 
are $(8480 \leq \lambda \leq 8570)$ and $(8640 \leq \lambda \leq 8684) \AA$, which we plot in Figure 3.3.

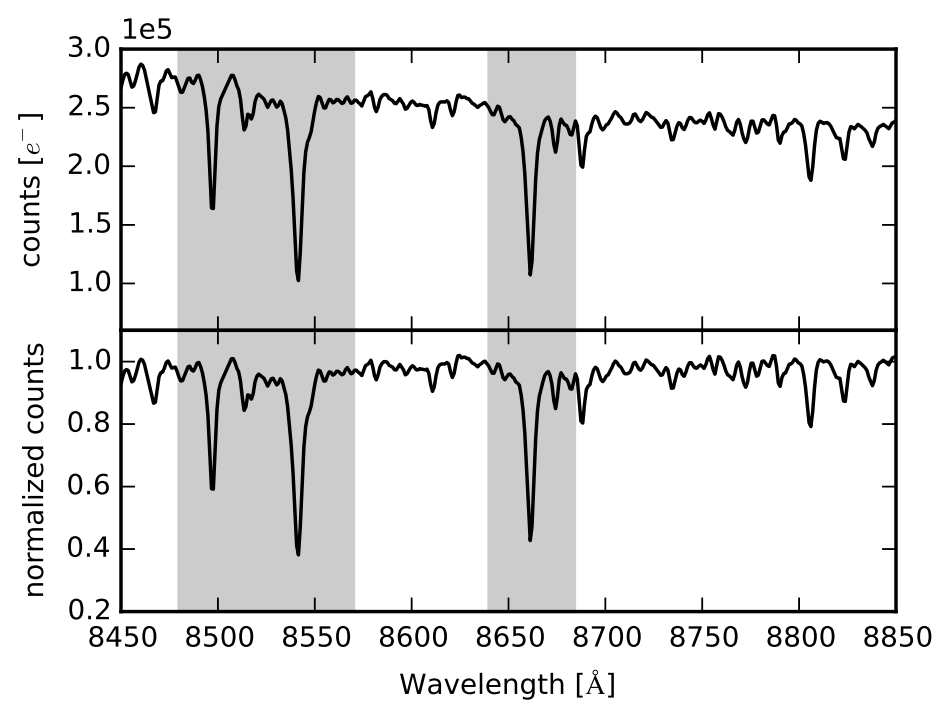

Fig. 3.3. - Top: trimmed spectrum of the RV standard HD 146051. The gray regions represent the spectral windows used for the cross-correlation, chosen to fully contain the CaT features and taken from the Calcium triplet bandpasses used in Chapter 2. Bottom: same spectrum, but after normalization by a low-order polynomial.

Next, these N-1 RV measurements for each standard were averaged, and its literature heliocentric velocity subtracted. We would expect the average-literature RV difference for each star to remain close to zero,but this is not always the case. If there were any star whose resultant RV difference, compared to the literature value, deviates largely from zero (defined as more than $5 \mathrm{~km} \mathrm{~s}^{-1}$ ), then we remove it from the set and repeat the process, until the RV differences in the whole set are small. In addition, we calculated an average Tonry and Davis $r$ value $(T D R)$ (Tonry \& Davis 1979) for each standard spectrum from the N-1 TDR values from the cross-correlation against the other templates. Finally, the template for the run was chosen as the one that has the largest average TDR, and ideally, whose deviation from the literature value was small. However, we do not set a strong constraint on the RV offset to be the smallest in the set, because we still can correct for any RV offset of the template 
later, by simply subtracting this offset from each object RV.

\section{Standard Stars and Radial Velocity Error Estimation}

We estimated the random errors of the RV measurements through the method presented by Frinchaboy \& Majewski (2008), which is based on Vogt et al. (1995). That process is explained briefly here.

The radial velocity $v$ of a star measured from a spectrum is assumed to have a $1-\sigma$ error that is nearly proportional to the inverse of the $(T D R)$ parameter (Tonry \& Davis 1979), this is:

$$
\sigma_{v}=\frac{\alpha}{1+T D R}
$$

where $\alpha$ is a constant of proportionality that is particular to each observing run, and can be calculated from spectra of standard stars with multiple observations through the equation:

$$
\alpha^{2}=\frac{\sum_{i} \sum_{j}\left(1+T D R_{i, j}\right)^{2}\left(V_{i, j}-\left\langle V_{j}\right\rangle\right)}{\chi_{50, n}^{2}}
$$

$V_{i, j}$ represents the $\mathrm{i}$-th observation of the $\mathrm{j}$-th star, whose $T D R$ value is $T D R_{i, j} \cdot\left\langle V_{j}\right\rangle$ is the average RV measured for the $\mathrm{j}$-th standard star, where each $i, j$ measurement is weighted by the inverse of $\left(1+T D R_{i, j}\right)$ squared. $\chi_{50, n}^{2}$ is the median $\chi^{2}$ for the $n$ degrees of freedom of the sample. The latter is defined as the difference between the total number of observations of standard stars in the sample and the number of unique standards. See Vogt et al. (1995) and Frinchaboy \& Majewski (2008) for further references. Figure 3.4 shows the set of standard stars and the derived 1- $\sigma$ error for stars with multiple observations. 


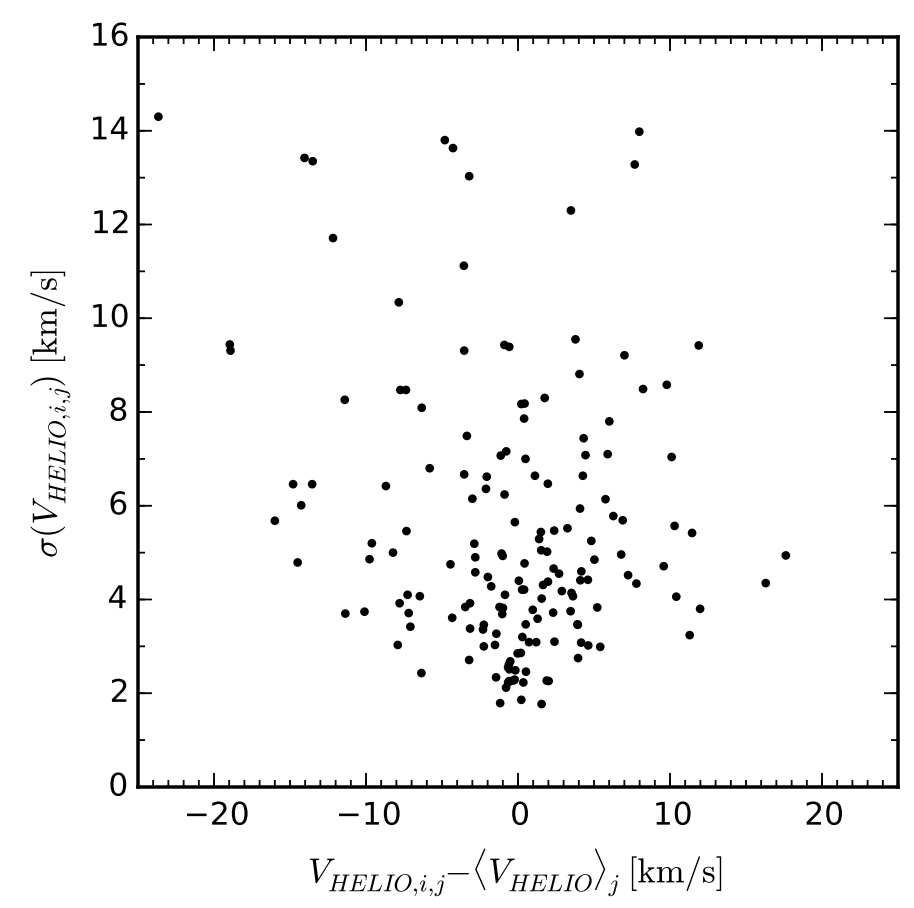

Fig. 3.4.- Measured heliocentric velocities and 1- $\sigma$ errors for stars with multiple observations. We calculate the average velocity $\left\langle V_{H E L I O}\right\rangle_{j}$ for the $j-t h$ star by weighting each $i-t h$ observation by the inverse of its error squared.

\section{Radial Velocity and Uncertainty Estimation for Objects}

For each observing run, we measured RVs for our science stars by cross-correlating our stellar spectra against the chosen RV standard star template. In practice, the templates are a single spectrum from an RV standard star for each run, and were selected as described in Section 3.2.3. Again, we set FXCOR to Fourier-filter and normalize both object and template spectra, and masked all the data along the spectral direction except for the calcium triplet lines. The output RVs are given in the heliocentric frame. We calculated the uncertainty for the velocities via Equation 3.1, using the TDR value measured by FXCOR for each object and the respective value of $\alpha$ derived for the template as described in Section 3.2.3. For stars with multiple obser- 
vations (i.e., multiple spectra collected), we combined the heliocentric radial velocities with a weighted average, where the weights were the inverse of the RV uncertainty squared for each measurement. The combined uncertainty is calculated as a weighted standard deviation. We excluded from the sample any star whose RV uncertainty was larger that $15 \mathrm{~km} \mathrm{~s}^{-1}$. Finally, we converted the radial velocities in heliocentric frame into velocities in the Galactic Standard of Rest (GSR) as follows:

$$
V_{G S R}=V_{\text {helio }}+\left(U_{\odot} \cos b \cos l+V_{\odot} \cos b \sin l+W_{\odot} \sin b\right)+V_{\text {Circ }} \cos b \sin l
$$

where $V_{\text {helio }}$ is the RV of a star in the heliocentric frame, $\left(U_{\odot}, V_{\odot}, W_{\odot}\right)$ is the solar peculiar motion in Galactic Cartesian $(U, V, W)$ coordinates, $V_{C i r c}$ is the circular velocity at Sun's position - the so-called Local Standard of Rest-, and $(l, b)$ are the Galactic longitude and latitude of the star, respectively.

We adopted the Galactic rotation and Solar motion values used by the Law \& Majewski (2010) model, for the most direct comparison of our observations and their model predictions (Section 3.3.2).

The radial velocities for all stars in the spectroscopic sample are presented in Figure 3.5. The position of the leading and trailing arms are made more evident by the coherent velocity trends apparent in panel (b) of Figure 3.5. However, we cannot clearly delineate these tidal structures until we weed out contamination from foreground Galactic M-dwarfs that may have slipped through the color-color selection. 


\subsubsection{Giant/Dwarf Separation}

Even though we used the photometric target selection criteria presented by Majewski et al. (2004), which discriminates M-giants from M-dwarfs in 2MASS color-color space, our sample may still suffer contamination from foreground Milky Way (MW hereafter) M-dwarfs. The color-color selection is sensitive to photometric errors, especially at the faint end of 2MASS. In this section, we describe the process followed to discriminate dwarfs and giants. In short, we exploit (1) the gravity sensitivity of the Na I doublet (Schiavon et al. 1997, SBR97 hereafter), and (2) reduced proper motion diagrams - a technique extensively used by Luyten (1922), where giants and dwarfs are separable through the differences in proper motion at fixed apparent magnitude. Neither method is fully definitive due to uncertainties, but can be combined statistically to achieve relatively reliable discrimination based on joint probabilities. However, we could not achieve conclusive results from these methods because of large uncertainties in the results. Therefore, we developed statistical estimators based on the work of Gilbert et al. (2006). Below, we describe these three methods and results.

\section{The Na I Doublet as a Gravity Discriminator}

One advantage of spectroscopic observations of late-type stars in the Ca II triplet region is the proximity of the Na I doublet $(\lambda(8183,8195) \AA)$. SBR97 have shown that $\mathrm{Na}$ I doublet is sensitive to surface gravity and temperature in late-type stars. The authors studied the behavior of this feature in cool stars by means of both

observed and synthetic spectra. SBR97 conclude that the doublet is suitable as a giant/dwarf discriminator for late-type stars.

The strength of the doublet, as for the Ca II triplet (Chapter 2), is measured in terms of the equivalent width $(W)$ from predefined line and continuum bandpasses 
(Section 2.2). SBR97 explore the behavior of the doublet with temperature across the range $\left[2,500<T_{\text {eff }}<5,500\right] \mathrm{K}$ for giants and dwarfs, and optimize the line and continuum bandpasses so that the impact of molecular bands is minimized. These molecular features (due to $\mathrm{TiO}$ ) appear in the later stellar types that they include in their sample.

The doublet strength, $W$, stays around $1 \AA$ for giants across the whole temperature range that SBR97 explore, while for dwarfs, $W$ decreases linearly from 9 to $3 \AA$ for $\left[2,500<T_{\text {eff }}<3,800\right] \mathrm{K}$, and then from 3 to $1 \AA$ for $\left[3,800<T_{\text {eff }}<5,500\right] \mathrm{K}$.

Because our spectra were intended for deriving radial velocities only, the SNR of the data is lower than what is usually required for measuring reliable equivalent widths. As a result, the quality for a fraction of our spectral sample is marginal for the purpose of measuring the equivalent width of the doublet to high accuracy. As a precautionary procedure, we measure $W$ using numerical integration, and line fitting, to compare if the results are consistent. The centers of the lines of the doublet are only $11 \AA$ apart; thus, the line fitting approach requires a fitting function that fits both lines simultaneously to recover fluxes accurately in the region where the line wings overlap (see bottom panel of Fig. 3.6). The profile of the doublet is described by two Gaussian profiles and a linear continuum as:

$$
f_{L}(\lambda)=f_{C}(\lambda)-A_{1} \exp \left[-\frac{1}{2}\left(\frac{\lambda-\lambda_{o, 1}}{\sigma_{1}}\right)^{2}\right]-A_{2} \exp \left[-\frac{1}{2}\left(\frac{\lambda-\lambda_{o, 2}}{\sigma_{2}}\right)^{2}\right]
$$

where $f_{C}(\lambda)$ is a linear function that describes the line continuum. The terms $A_{1}$ $\left(A_{2}\right), \sigma_{1}\left(\sigma_{2}\right)$ and $\lambda_{1}\left(\lambda_{2}\right)$ represent the peak intensity, width and line center for the component 1 (2), respectively. For the line-fitting method, we use the bandpass used by SBR97 for line integration. This bandpass discards the right end of the doublet, 
which sometimes is affected by an unidentified spectral feature ${ }^{4}$ and increases the uncertainty of the fit. However, the line fit does not take into account the region in between the lines, because it also introduces noise into the fit. The bandpasses for both approaches are given in Table 3.2. For the line integration approach, we test a modified bandpass because the standard SBR97 blue end introduced noise given that the line strength is low. Therefore, we shorten the blue end of the bandpass by $4 \AA$, and increase the red end by $2 \AA$ to include some of the line signal lost. Extending the bandpass farther into the red introduces noise again. This revised bandpass range is better centered around the lines. Figure 3.6 shows spectra of the doublet for a giant and a dwarf star. The figure includes the continuum and line bandpasses, as well as the fitted continuum and fitted line profile that results from Equation 3.4. In both cases, we measure $W$ by integrating Equation 2.1. In the case of numerical integration, the line profile is given by the data within the line bandpass, and integration of Equation 2.1 is done using Simpson's rule to compute an integral from samples, implemented through the scipy.integrate library in Python.

Figure 3.7 presents a comparison between the results from both methods. We find that the profile fitting and the numerical integration methods give similar, but not identical, results. For some stars, the numerical integration method results in a negative value of $W$. This is caused by noise in the spectra, where there is, by chance, pixels with a preponderance of values above the estimated continuum. By definition (Eq. 2.1), the integration of those pixels results in negative values. In addition, $W$ from numerical integration increases faster than the value from line fitting. This is likely caused by the extended red-end of the integration bandpass used for the former, where the red line of the doublet maintains large depth. We also present a histogram

\footnotetext{
${ }^{4}$ SBR97 identifies a V I line around $8199 \AA$, however, they use high-resolution spectrum to see this feature, which is not distinct in our spectra.
} 
Table 3.1. Spectroscopic observing runs

\begin{tabular}{|c|c|c|c|c|}
\hline Telescope & UT date & Object spectra & Spectrograph & Spectral coverage $[\AA]$ \\
\hline KPNO 2.1-m & 2004, April 18-26 & 352 & GoldCam & $7170-9130$ \\
\hline KPNO Bok 2.3-m & 2003, May 21-23 & 72 & Boller \& Chivens & - \\
\hline CTIO $1.5-\mathrm{m}$ & 2003, June 19-22 & 194 & Cassegrain & $7840-9160$ \\
\hline KPNO Bok $2.3-\mathrm{m}$ & 2003, December 14, 16-19 & 215 & Boller \& Chivens & $7500-9160$ \\
\hline CAHA $3.5-\mathrm{m}$ & 2004, January 19-22, 24-26 & 96 & TWIN $^{\mathrm{a}}$ & $7460-9050$ \\
\hline KPNO 4-m & 2004, February 13-16 & 130 & R-C & $6560-9120$ \\
\hline CAHA 3.5-m & 2004, July 15-17, 23-24 & 25 & TWIN a & $7460-9050$ \\
\hline CTIO 4-m & 2004, August $23-27$ & 93 & $\mathrm{R}-\mathrm{C}$ & $6350-8870$ \\
\hline CTIO 1.5-m & 2004, October 8-13 & 141 & Cassegrain & $7630-8940$ \\
\hline MMT 6.5-m & 2014, Jan 06 & 39 & Red Channel & $8100-8800$ \\
\hline
\end{tabular}

${ }^{a}$ Only red-channel data used

Table 3.2. Na I line fit, integration and continuum bandpasses $[\AA]$ used in this work.

\begin{aligned} & \hline \hline Line center \multicolumn{1}{c}{ Line Bandpass } \\ & \hline Center line 1$\lambda_{1}=8183.25 \\ &$ Center line 2$\lambda_{2}=\frac{8194.79+8194.84}{2} \\ &$ Integration bandpass (num. int.) $8176.0-8199.0 \\ &$ Integration bandpass (line fit) $8172.0-8197.0 \\ &$ Fit bandpass $8172.0-8187.0,8190.0-8197.0 \\ &$ Blue continuum $8170.5-8174.0 \\ &$ Red continuum $8233.5-8235.2 \\ &$ Continuum linebandpass $8177.0-8198.0 \\ &$ Continuum SNR bandpass $8080.0-8170.0 \\ &$\hline\end{aligned}


of the results in Figure 3.8. Stars with $W<1.1 \AA$ are likely giants. However, some of these results have non-negligible uncertainties (e.g., as seen in the bottom panel of Fig. 3.7). For this reason, it is worthwhile to try alternative methods to discriminate giants and dwarfs. 


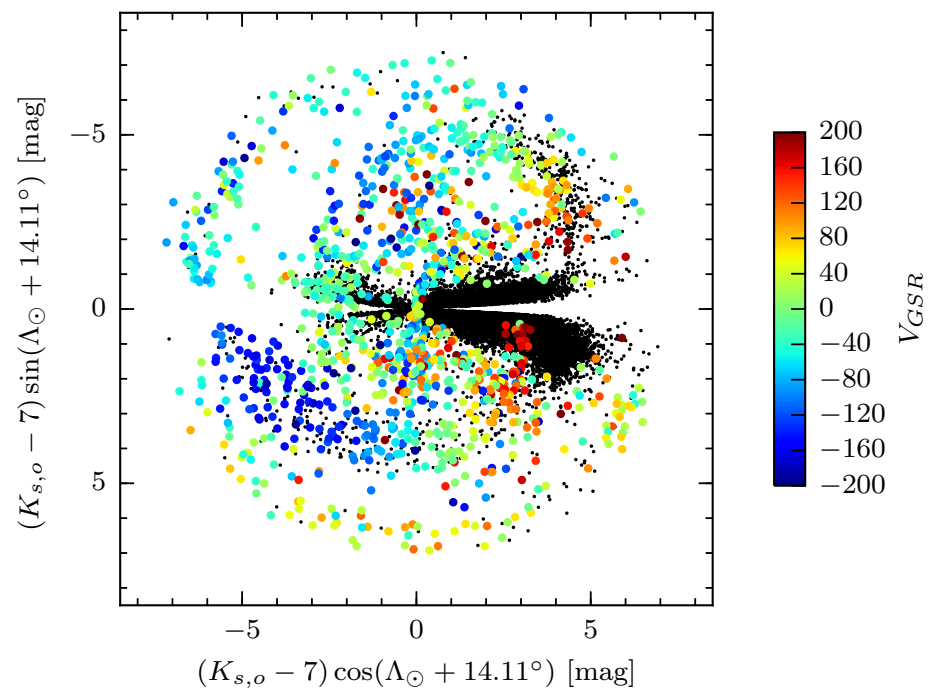

(a) Same as Figure 3.2, but stars with spectroscopic measurements are color-coded by radial velocity in the GSR.

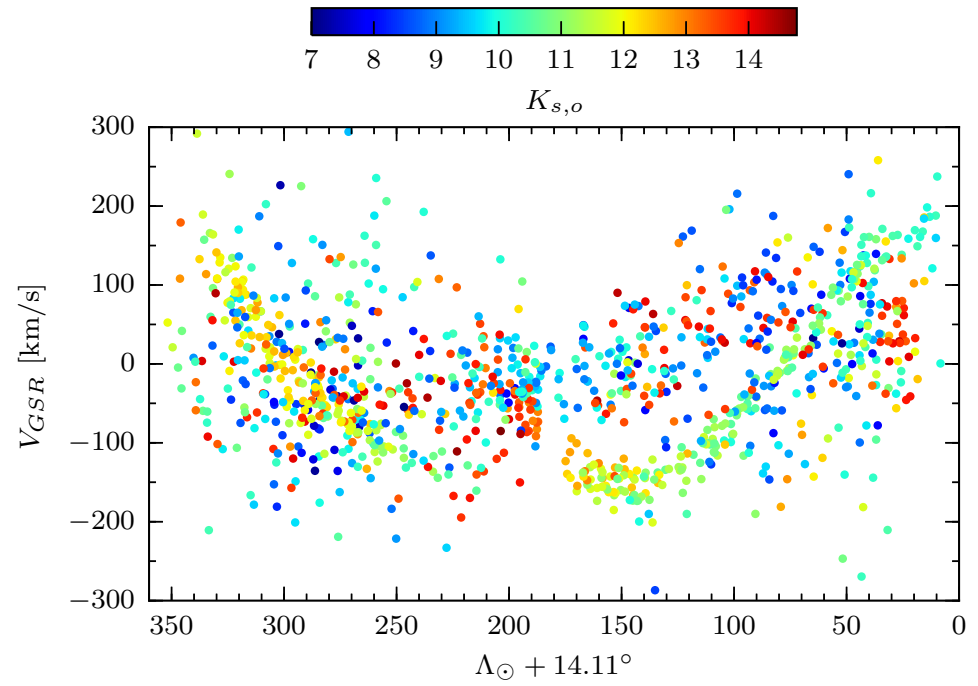

(b) Same data as in panel (a), but in the Sgr longitude $\Lambda_{\odot}$ versus GSR radial velocity plane and color-coded by $K_{s, o}$ magnitude. The tidal tails form coherent structures in this plane, which can be used to trace positions of the trailing and leading arms qualitatively in the figure. The leading arm extends from $(\Lambda+$ $\left.14.11^{\circ}, V_{G S R}\right) \sim(330,125)$ down to $(240,-100)$, where it reaches the Galactic plane. whereas the trailing arm extends from $\sim(10,175)$ to $\sim(180,-100)$.

Fig. 3.5. - Radial velocities of the whole spectroscopic sample in the GSR. We have included the stars of Majewski et al. (2004). 


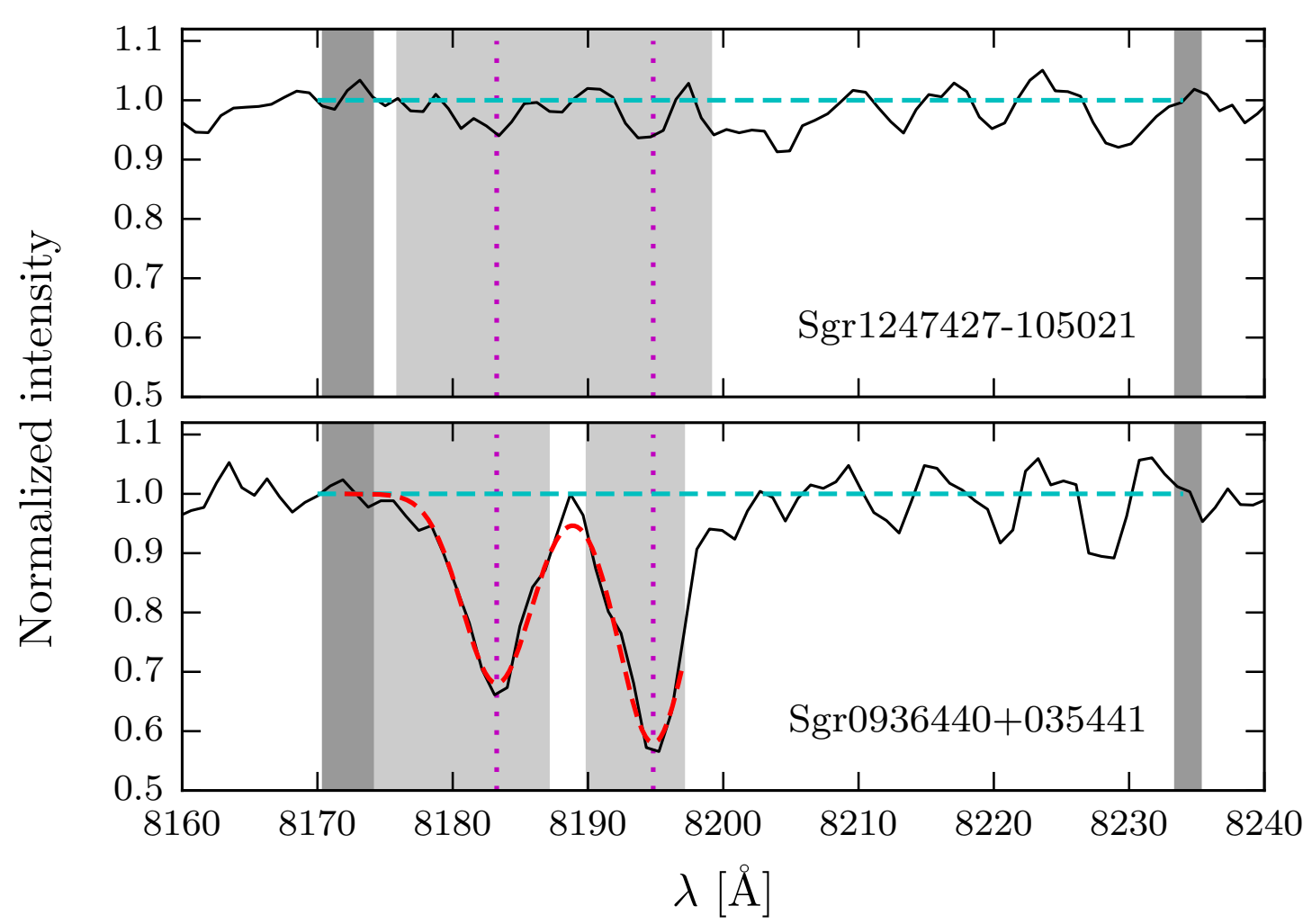

Fig. 3.6. - Spectra of a giant (top) and a dwarf (bottom) star showing the sensitivity of the $\mathrm{Na}$ I doublet to gravity. The magenta lines indicate the line centers. The dark shaded regions show the continuum bandpasses. The cyan line shows the fitted continuum. In addition, we illustrate the bandpasses for the numerical and line-fitting procedures. In the top panel, the line bandpass (in light gray) is used for numerical integration. In the bottom panel, the fitted bandpass is indicated by the two light gray bands, and the profile used for the integration - the best-fit result - is shown by the red dashed line. 


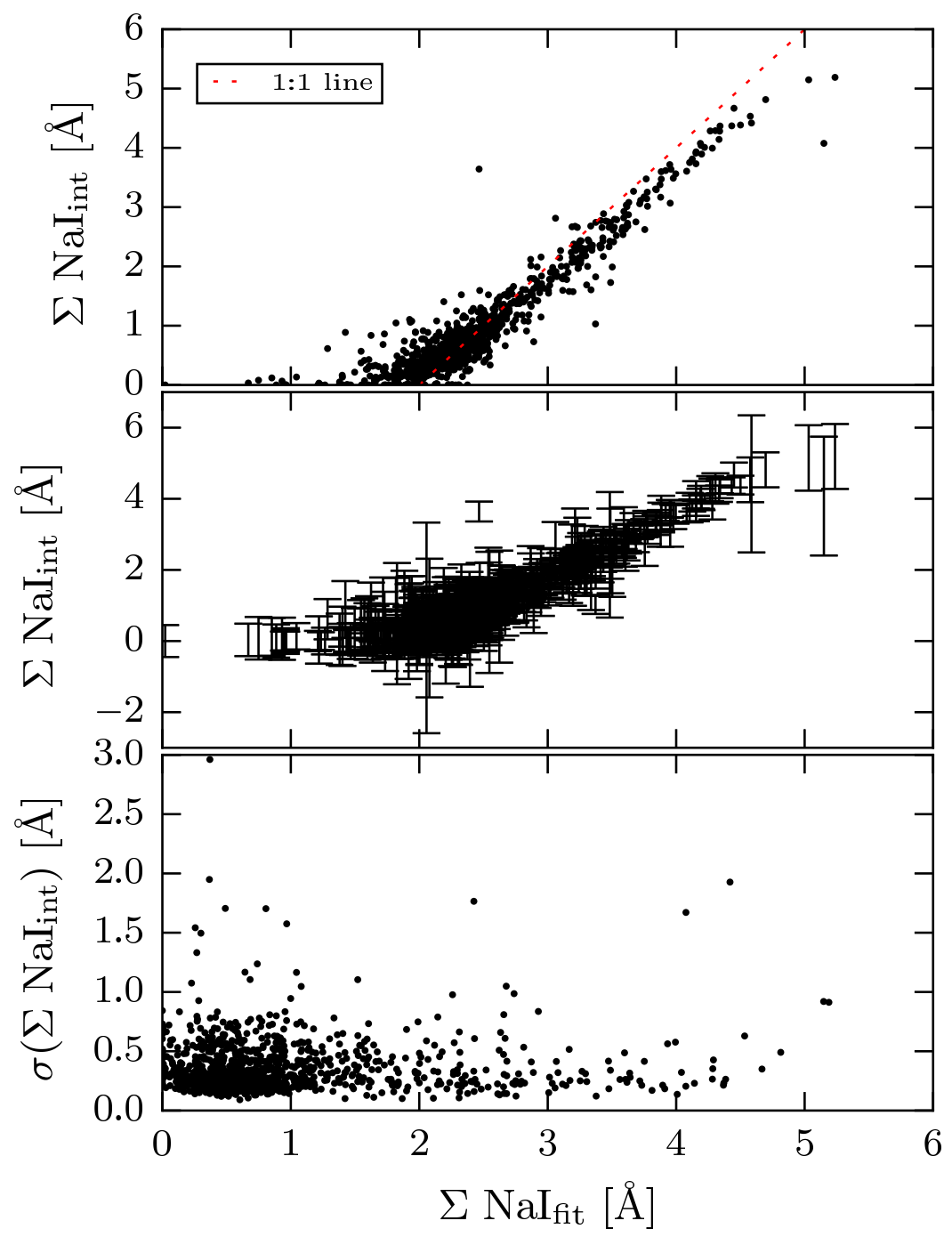

Fig. 3.7.- Results for the Na I equivalent width $(W)$ for the spectroscopic dataset. The upper and middle panels compare results between the profile fitting (fit) and numerical integration (int) methods. The dotted red line illustrates the 1:1 relation. The middle panel shows the estimated uncertainties for the fitted profiles. The lower panel shows the uncertainties derived by line integration as a function of equivalent width. 


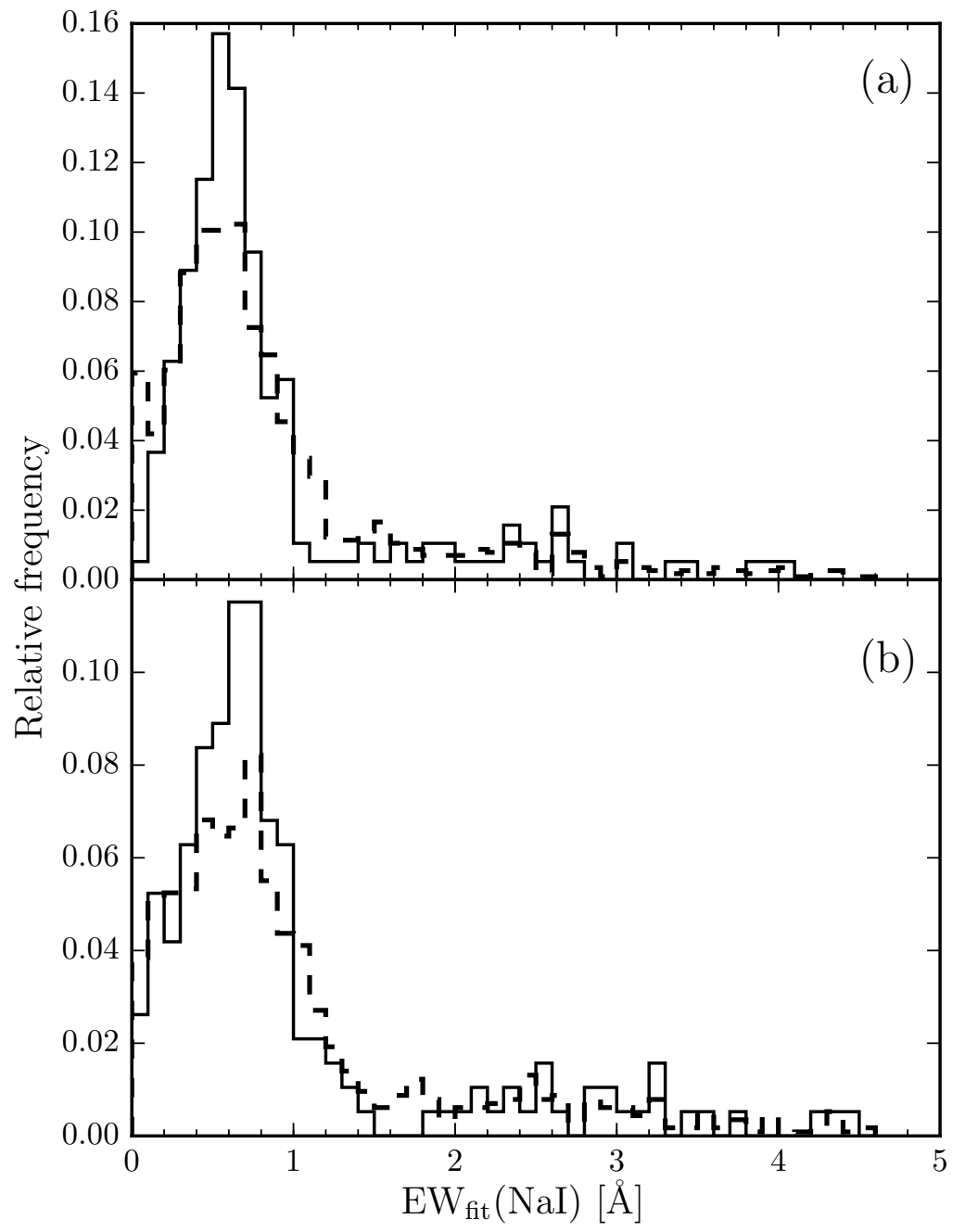

Fig. 3.8. - Na I line strengths from (a) line fitting, and (b) line integration. Measurements with $\sigma \leq 0.25[\AA]$ are shown with solid lines, and all measurements are shown with dashed lines. 


\section{Reduced Proper Motion Diagrams as a Giant/Dwarf Discriminator}

A non-negligible fraction of the spectroscopic sample have large uncertainty in $W(N a I)_{f i t}$.

For instance, $13 \%$ of the sample has $\sigma\left(W(N a I)_{f i t}\right)>0.5 \AA$. As a result, obtaining a clear giant/dwarf discrimination using the $\mathrm{Na}$ doublet may not produce a completely clean giant/dwarf separation on its own. Furthermore, we could not recover the archived spectra of one observing run (May 2003, see Table 3.1) that has radial velocities measured for 72 program stars. For these reasons, we explored reduced proper motions as a second method to separate dwarfs from giants.

Following Luyten (1922), we define the "Reduced Proper Motion" (RPM hereafter) as:

$$
H_{K_{s}}=K_{s, o}+5+5 \log \mu
$$

where $K_{s, o}$ and $\log \mu$ terms are the dereddened $K_{s}$ magnitude and the proper motion modulus, respectively. The latter is calculated from the proper motion components along right ascension and declination. Equation 3.6 is equivalent to:

$$
H_{K_{s}}=M\left(K_{s, o}\right)+5 \log v_{t}+3.38
$$

where $M\left(K_{s, o}\right)$ and $v_{t}$ are the absolute magnitude and transverse velocity of the star. RPMs have proven useful (Luyten 1922) for stellar luminosity discrimination because we expect stars in the Galaxy to have a limited range of transverse velocities. As a consequence, differences in $H_{K_{s}}$ will be a result of the distinct absolute magnitudes of giants and dwarfs, so that $H_{K_{s}}$ resembles absolute magnitude. It is usual to combine the RPM with color information to construct a "reduced proper motion diagram" (RPMD) (e.g., Jones 1972; Lépine et al. 2003), which resembles a color-magnitude 
diagram, with apparent magnitude sorted by absolute magnitude via proper motions. We construct and explore this diagram for our data below.

We obtained proper motion data from the PPMXL catalog (Roeser et al. 2010), by matching the equatorial coordinates of our target M-giant candidates against the catalog. PPMXL is an all-sky catalog of absolute proper motions in the ICRS system (Arias et al. 1995) that combines USNOB-1.0 (Monet et al. 2003) and 2MASS (Skrutskie et al. 2006) astrometry.

Because PPMXL-matched data contain the 2MASS photometry, we checked the photometry of the original M03 catalog used to select the spectroscopic targets in our sample. We dereddened the sources following the steps described in Section 3.2.1. Figure 3.9 compares the original (old) photometry — used by our group to select Sgr candidates - and the updated (new) 2MASS photometry. The difference for stars at the faint end is explained by the fact that M03 selected stars from a proprietary, pre-release version of $2 \mathrm{MASS}$, though they state using a "final processing" of the catalog (the final version of 2MASS was released in March 2003.) To explore the significance of the magnitude updates in later 2MASS versions, we plot the M03 selection criteria applied to both photometric sets in Figure 3.10, color-coded by $\mathrm{Na}$ I $W$. It is clear that most of the stars we find to have strong sodium absorption -i.e., likely dwarfs - land outside the nominal color-color giant star selection box originally utilized for this work. We proceed with the updated version 2MASS photometry for the remaining fraction of this Chapter. As we shall see, the systematic errors in the original photometry explains many of the faint, "distant" structures originally identified by M03 (see Fig. 3.9).

We present the RPMD for our spectroscopic sample in Figure 3.11. We explore the sensitivity of the RPMD to luminosity class by separating the sample by sodium 


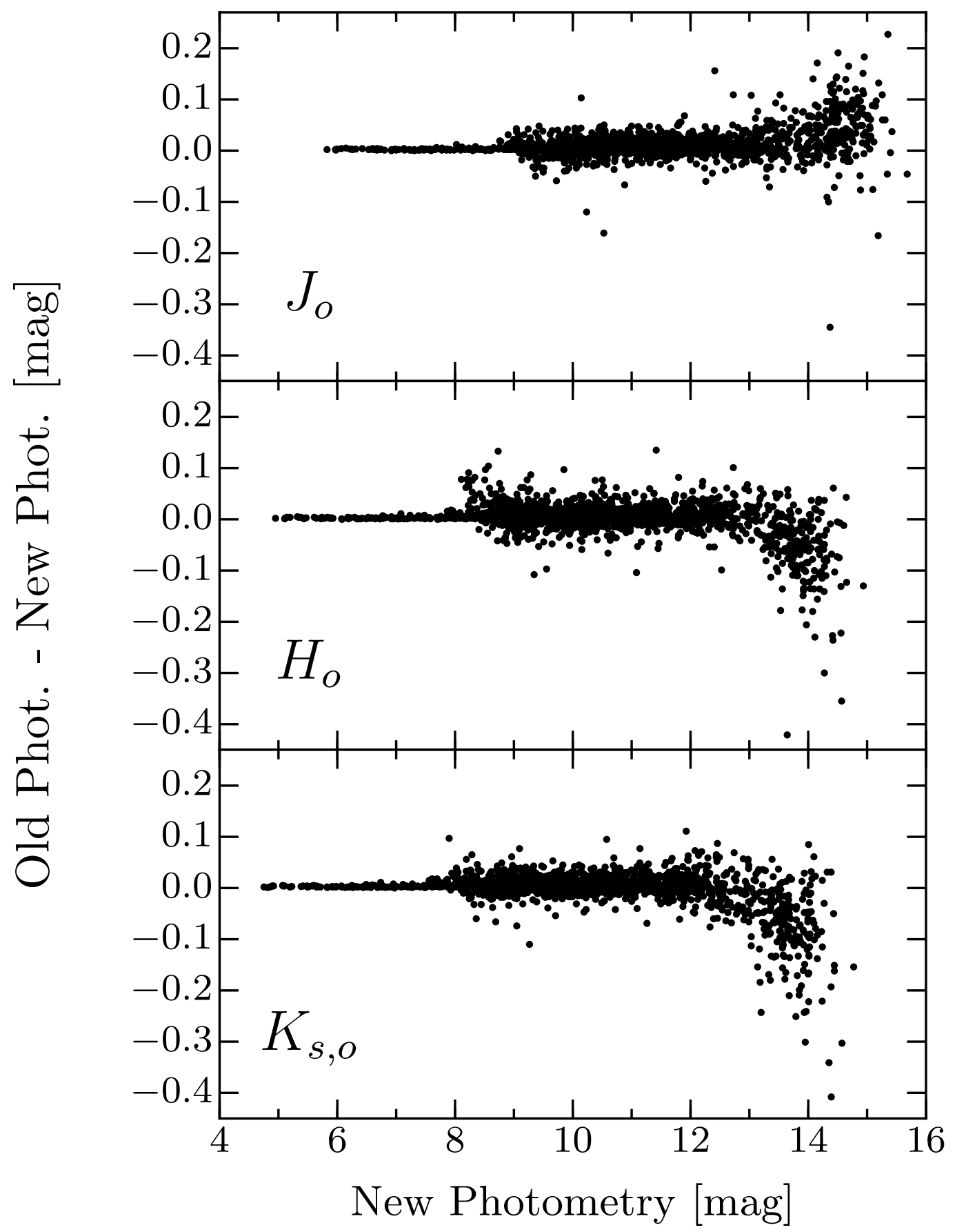

Fig. 3.9.- Comparison of the 2MASS photometry used by Majewski et al. (2003) for the original target selection (Old Phot.) and updated (All-Sky Data Release, March 2003) 2MASS photometry. 

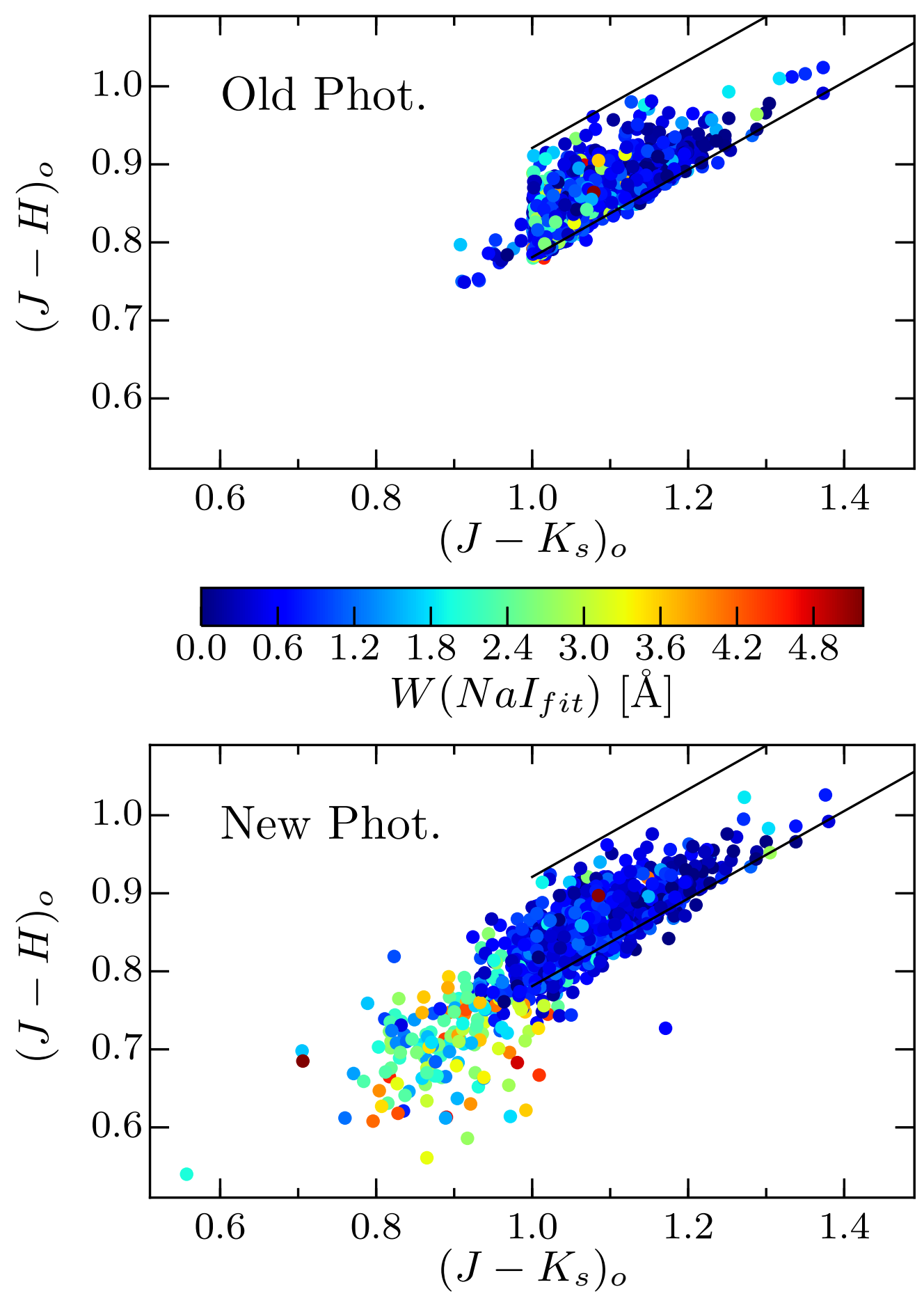

Fig. 3.10.- Comparison of the sample selection using the 2-color criterion of Majewski et al. (2003), for the original - i.e., "pre-release" — and updated All-Sky Data Release 2MASS photometry. With the updated photometry, most stars strong in sodium absorption - likely dwarfs - are outside of the region targeted for the Mgiant candidate selection. 

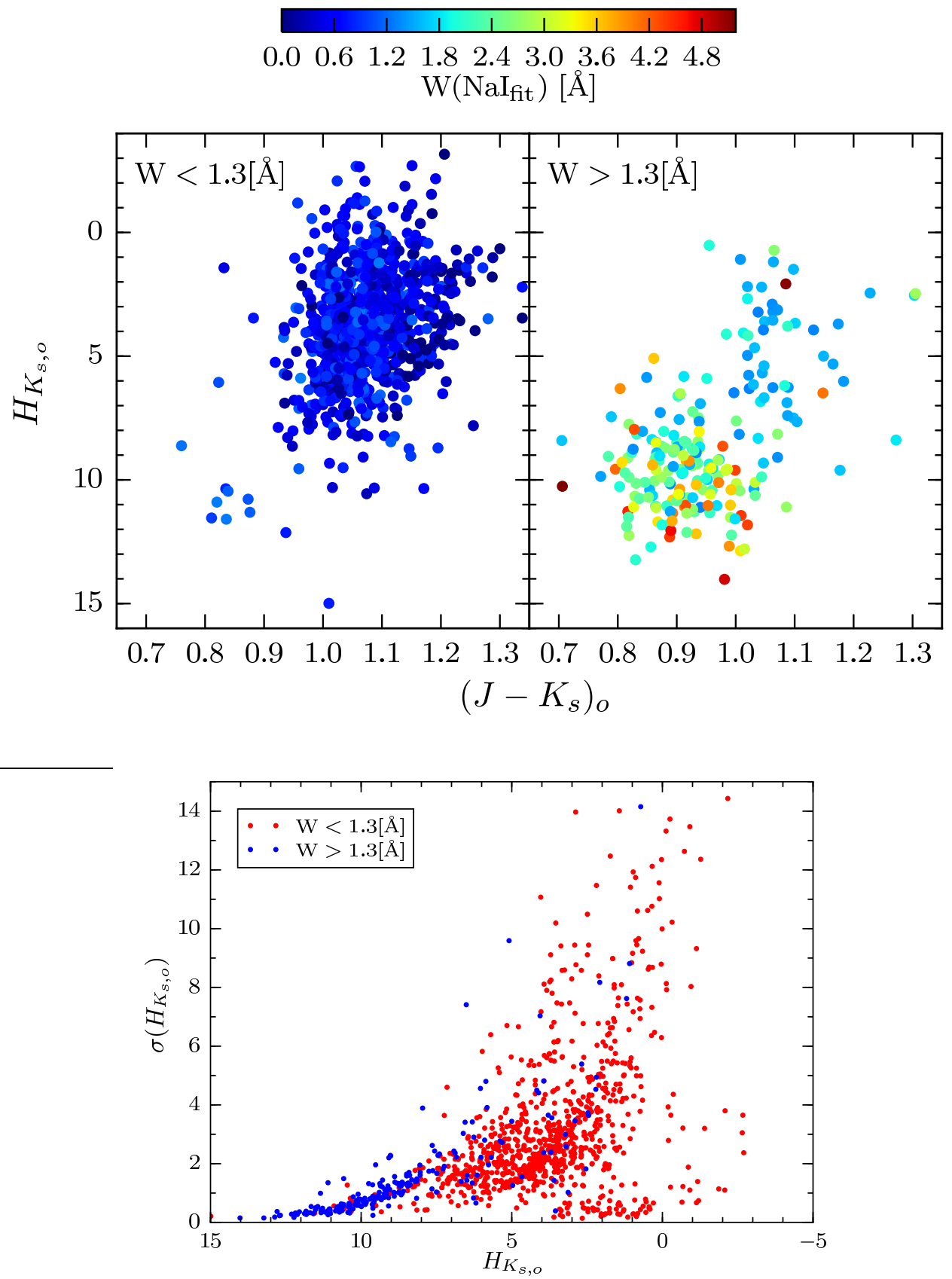

Fig. 3.11. - Reduced proper motions using PPMXL proper motions and the updated photometry. Top panels: RPMD with stars color-coded by NaI I strength. We plot the sample with $W\left(N a I_{f i t}\right)<1.3$ - likely giants - in the left panel, while likely dwarfs are in the right panel. Most stars in these two subsamples occupy distinct loci in the RPMD. However, there are some stars that have $W>1.3$ but reside in the locus of giants, and stars with $W<1.3$ that lie in the locus of dwarfs. This may be produced by errors in the RPM or sodium equivalent widths. Bottom panel: Reduced proper motion uncertainties, as a function of RPM. Likely-giants and -dwarfs are colored by their sodium strength, using the selection from the upper panel. Stars with small $H_{K_{s}}$ tend to have small $\mu$ and so are most affected by the proper motion errors. 
strength. We set the giant/dwarf boundary to $W=1.3 \AA$, which corresponds, approximately, to the upper boundary of the peak produced by likely giants in Figure 3.8 - and in agreement with the values given by SBR97. We find that RPMs correlate with $\mathrm{Na}$ I equivalent width in our sample. However, the RPMs have large uncertainties which particularly affects small $H_{K_{s}}$ (Fig. 3.11), which particularly affects small $H_{K_{s}}$. Because of this complication, we adopt an statistical approach that we introduce in the next subsection.

\section{Statistical Approach to the Giant/Dwarf Discrimination}

We have seen that sodium strength (SBR97), RPMs (Luyten 1922), and the 2MASS 2-color space (M03) can each be used as gauges for giant/dwarf discrimination. However, each of these methods are affected by measurement uncertainties that make it difficult to obtain a clean sample of giants, or to set a hard boundary between giants and dwarfs with each method alone. Therefore, we seek an approach that statistically combines the gravity-sensitive information contained in these three methodologies explored above. One method for doing this is presented by Gilbert et al. (2006), who sought K-giants in M31, but needed to weed out foreground MW dwarfs. Gilbert et al. (2006, G06 hereafter) utilized a different parameter space for their selection (e.g., different photometric criteria). However, we can implement their prescription to construct analytical Probability Distribution Functions (PDFs) in 1D and 2D from samples in our dataset. At the end of this section, we evaluate these PDFs to find the probability that a star is a giant or a dwarf. Below, we first explain the rationale of G06, and then we construct the actual PDFs to execute the giant/dwarf selection for our dataset.

Following G06, we construct a series of 1D and 2D PDFs or "diagnostics" —as 
G06 call each combination of parameters used in their PDFs. These PDFs are made for giants and dwarfs separately, but span the same parameter space. The method uses empirical training sets, constructed using a subset of the data under study that can be selected as giants or dwarfs with high confidence. After selecting these training sets, we construct 1D PDFs by fitting the Cumulative Distribution Function of stars in the training sets. To create a 2D PDF, G06 use the relation:

$$
P D F(x, y)=C P(x) \exp \frac{-0.5\left[y-\langle y\rangle_{f i t}(x)\right]^{2}}{\left[\sigma_{f i t}^{y}(x)\right]^{2}},
$$

where $P(x)$ is the 1D PDF calculated for the x-variable used in the diagnostic. The constant $C$ is a normalization factor so that the PDF integrates to 1.0 within the defined domain of $x$ and $y$. The quantities $\langle y\rangle_{f i t}(x)$ and $\sigma_{f i t}^{y}(x)$ are the mean and standard deviation, respectively, of Gaussian functions piecewise-fitted to data in the $y$-dimension, for bins in the $x$-dimension.

Equation 3.7 is used to construct several diagnostics from a combination of data dimensions where dwarfs and giants occupy separate loci. Specifically, for our analysis we adopt some combination of $\left(J-K_{s}\right)_{o},(J-H)_{o}, W\left(N a I_{f i t}\right)$, and $H\left(K_{s, o}\right)$.

Then, the probability for the $i$-th star to be a giant or a dwarf, from the $j$-th diagnostic is:

$$
L_{i j}=\log \left(\frac{P_{i j, g i a n t}}{P_{i j, d w a r f}}\right),
$$

where $P_{\text {giant }}$ and $P_{\text {dwarf }}$ denote the probability from the diagnostic calculated from the giant and dwarf PDFs, respectively.

After obtaining probabilities from Equation 3.8 for all diagnostics for the $i$-th star, the overall likelihood is defined as: 


$$
\left\langle L_{i}\right\rangle=\frac{\sum_{j} w_{j} L_{i, j}}{\sum_{j} w_{j}},
$$

where the weighting factor $w_{j}$ is usually set to unity for all diagnostics, except if the star lands in a region with poor sampling of the PDF for giants and dwarfs - in which case, "down weighting" (G06) is applied.

Downweighting of a diagnostic occurs if $P_{i j, g i a n t}<3 \sigma_{j, g i a n t}$ and $P_{i j, d w a r f}<3 \sigma_{j, d w a r f}$, where $\sigma_{j, \text { giant }}$ and $\sigma_{j, d w a r f}$ are the probability levels that include $90 \%$ of stars in the respective training sets. The downweighting relation is:

$$
w_{j}=\frac{w_{o}}{\left(\sigma_{j} / P_{j}\right)_{\text {giant }}^{2}+\left(\sigma_{j} / P_{j}\right)_{d w a r f}^{2}}
$$

where $w_{o}=2 / 9$ prevents $w_{j}$ from becoming larger than 1.0 for a downweighting threshold set to $3 \sigma$. In addition, we cap $\left\langle L_{i}\right\rangle$ to \pm 4 . This prevents a single diagnostic from overturning the others. In the end, a star with $\left\langle L_{i}\right\rangle>0(<0)$ is flagged as a giant (dwarf).

The complete dataset has 1,497 unique stars (including spectra from the observing run of Majewski et al. (2004)). Within the catalog, 1,140 stars have Na I and RPM measurements. We make a first training set (TS1 hereafter) that uses only stars that we select as high-quality by requiring that uncertainties in Na I and RPM are low (with thresholds specified below.) Afterwards, we create a second training set (TS2) using stars with medium-quality measurements. Even though the uncertainty in parameters for selection is larger, these stars sample the parameter space better. The stars in the TS2 are picked by applying the diagnostics calibrated with TS1. Finally, we perform the giant/dwarf separation for the complete sample using the diagnostics calibrated with stars from TS2.

Stars are selected for TS1 if they have $\sigma\left(W\left(N a I_{f i t}\right)\right)<0.25 \AA$ and $\sigma\left(H_{K_{s, o}}\right)<$ 
0.35. Following SBR97 and given the small uncertainty in this subset, we consider stars with $\left.W\left(N a I_{f i t}\right)\right)<1.3 \AA$ as giants, and dwarfs otherwise. With these criteria, TS1 has 46 stars (20 dwarfs and 26 giants). We construct 1D PDFs for dwarfs and giants in $\left(J-K_{s}\right)_{o}$ and $W\left(N a I_{f i t}\right)$. We test one and two Gaussian functions to describe the PDFs, to find an optimal fit describing the observed distributions. The PDFs are shown in Figures 3.12 and 3.13.

Next we construct 2D PDFs based on the two 1D PDFs and Equation 3.7. We explore the planes defined by $\left[\left(J-K_{s}\right)_{o},(J-H)_{o}\right],\left[\left(J-K_{s}\right)_{o}, W\left(N a I_{f i t}\right)\right],[(J-$ $\left.\left.K_{s}\right)_{o}, H_{K_{s, o}}\right],\left[W\left(N a I_{f i t}\right), H_{K_{s, o}}\right]$. The data and the fitted PDFs are shown in Figures $3.14-3.16$.

Next, we select stars for Training Set 2 (TS2) if they have $\sigma\left(W\left(N a I_{f i t}\right)\right)<0.35$ $\AA$ and $\sigma\left(H_{K_{s, o}}\right)<0.8$. However, this time we flag them as giants or dwarfs by evaluating the 2D PDFs for all diagnostics defined using TS1, and calculate the overall giant/dwarf probability via Equations 3.8, 3.9 and 3.10. With these considerations, TS2 has 133 stars total, where 68 are giants and 65 are dwarfs. We do not restrict TS1 stars to be selected for TS2 if the required criteria are met.

We repeat the calculation of 1D PDFs and 2D PDFs, following exactly the same steps as for TS1. TS2 samples the parameter space better than TS1. Results for the TS2, 1D PDFs are presented in Figures 3.18 and 3.19. The 2D PDFs calculated using TS2 are shown in Figures 3.20-3.23.

Finally, we use the TS2 PDFs to evaluate the giant/dwarf likelihoods for the complete dataset of stars using the four diagnostics, and then calculate the overall giant/dwarf probability for each star. If a star has no measurements for a given diagnostic - for example, no Na I strength - then we use the remaining diagnostics available. As a result, 1233 stars are selected as giants, and 233 as dwarfs. The other 
$\mathrm{NaI}_{\text {fit }} 1 \mathrm{~d}$ dist, dwarfs. T.S. 1

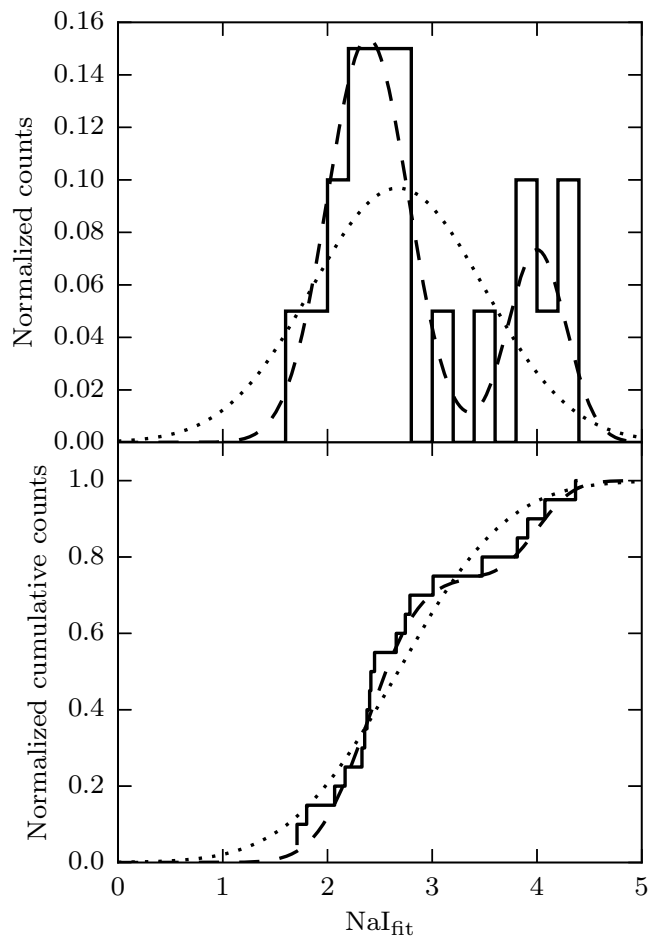

$\mathrm{NaI}_{\text {fit }} 1 \mathrm{~d}$ dist, giants. T.S. 1

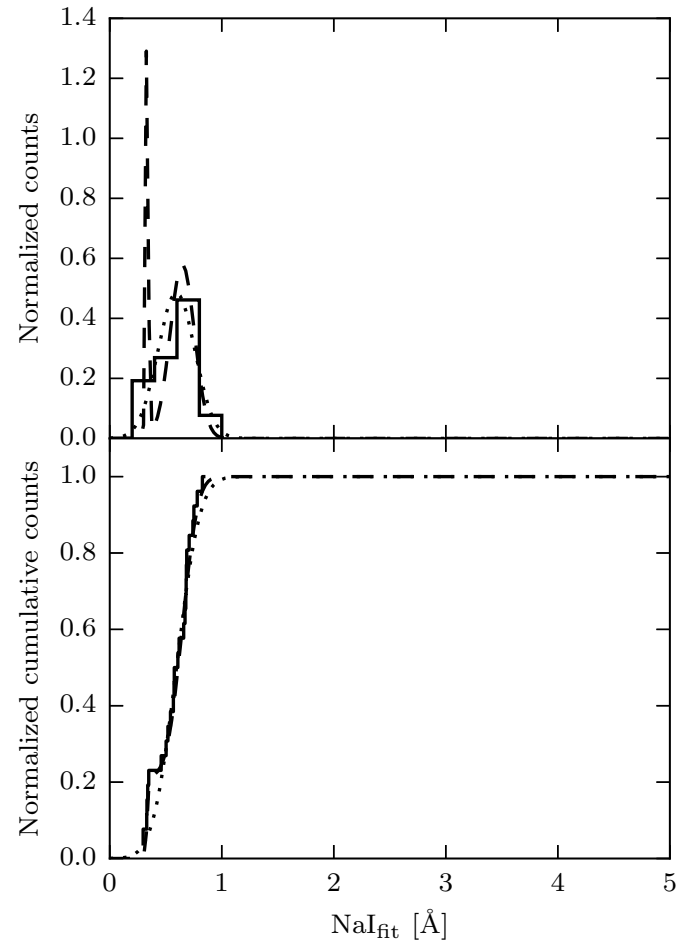

Fig. 3.12. - The 1D Probability Distribution Function (PDF) for dwarf (left column) and giant (right column) stars in Training Set 1 (TS1). The upper panels show the histogram and fitted single (double) component Gaussian as a dotted (dashed) curve, whereas the lower panels show the Cumulative Distribution Functions - used for the fit - with the same single and double component Gaussian functions. 
$\left(\mathrm{J}-\mathrm{K}_{\mathrm{s}}\right)_{\mathrm{o}} 1 \mathrm{~d}$ dist, dwarfs. T.S. 1

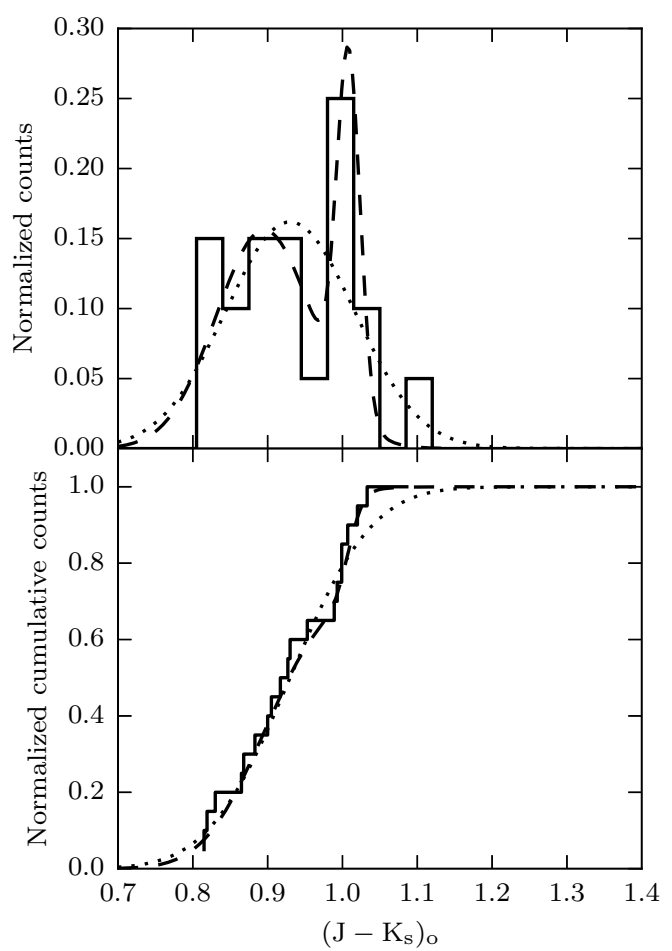

$\left(\mathrm{J}-\mathrm{K}_{\mathrm{s}}\right)_{\mathrm{o}} 1 \mathrm{~d}$ dist, giants. T.S. 1

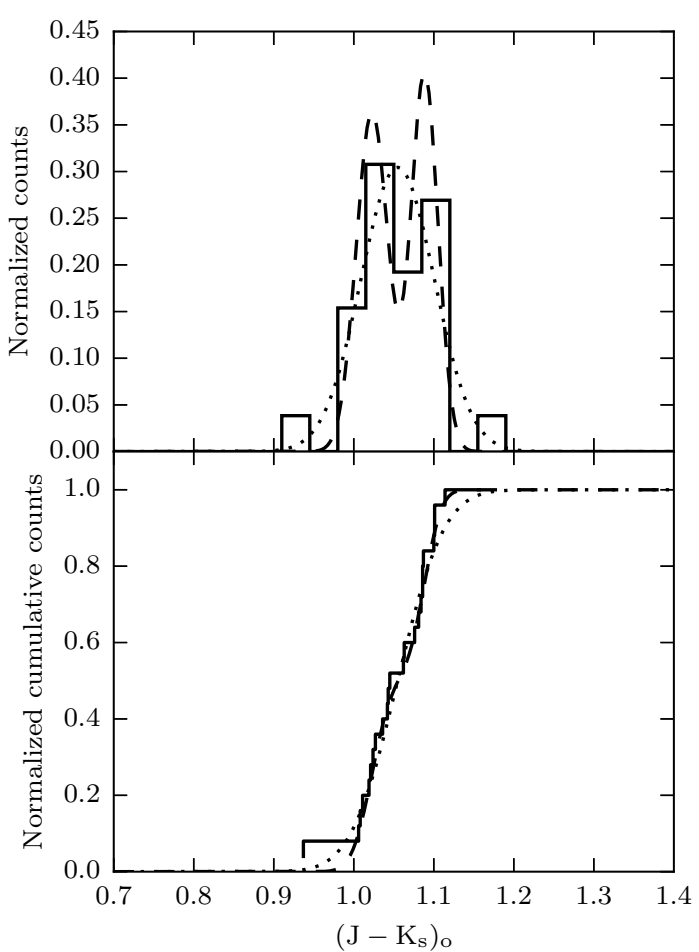

Fig. 3.13. - Same as Figure 3.12, for $\left(J-K_{s}\right)_{o}$. 
$2 \mathrm{~d}$ distribution for dwarfs. T.S. 1

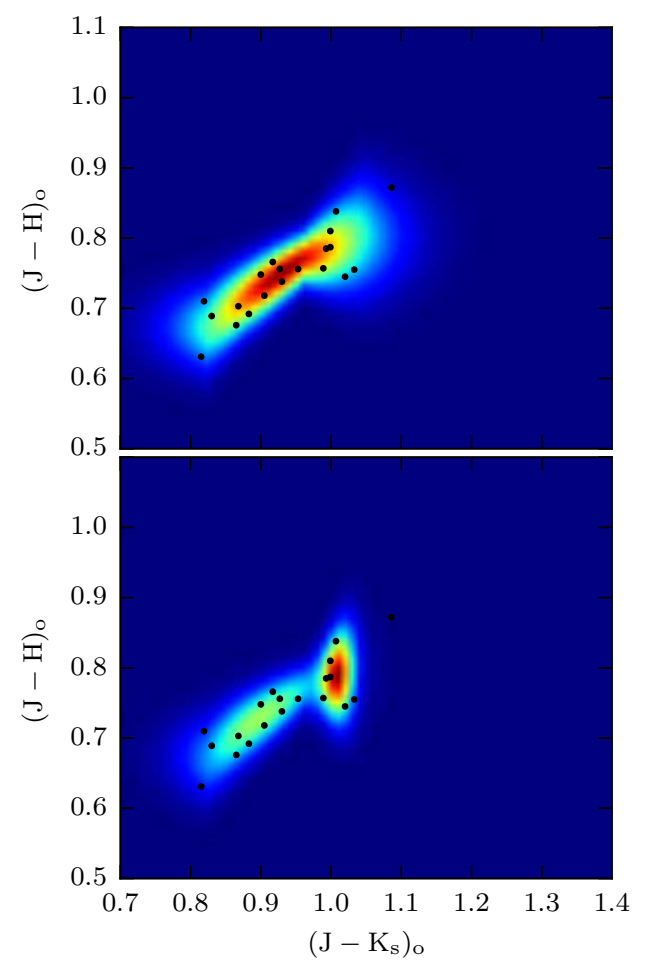

$2 \mathrm{~d}$ distribution for giants. T.S. 1

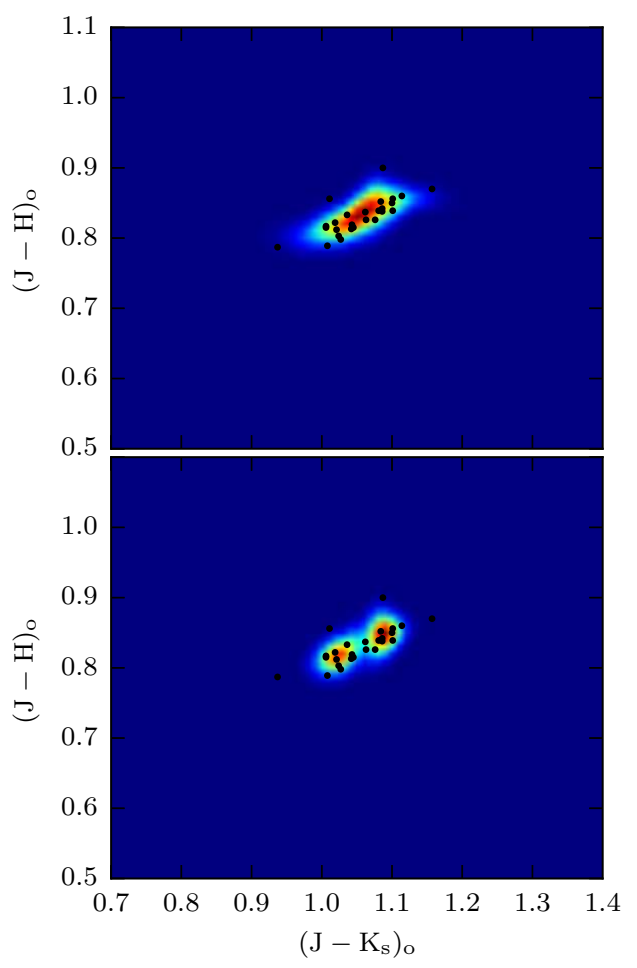

Fig. 3.14. - The 2D PDF and TS1 data for the $\left[\left(J-K_{s}\right)_{o},(J-H)_{o}\right]$ diagnostic. The panels in the left and right columns show the PDFs for dwarfs and giants, respectively. The top panels show the PDFs obtained from a single-Gaussian 1D PDF for $\left(J-K_{s}\right)_{o}$, while the bottom panels use a two-component Gaussian 1D PDF. 
$2 \mathrm{~d}$ distribution for dwarfs. T.S. 1

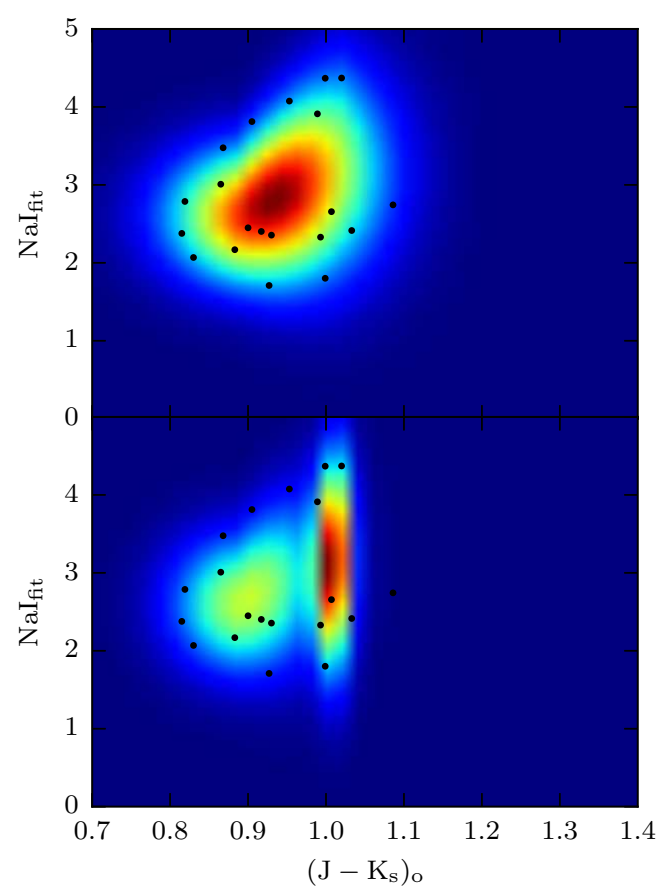

$2 \mathrm{~d}$ distribution for giants. T.S. 1

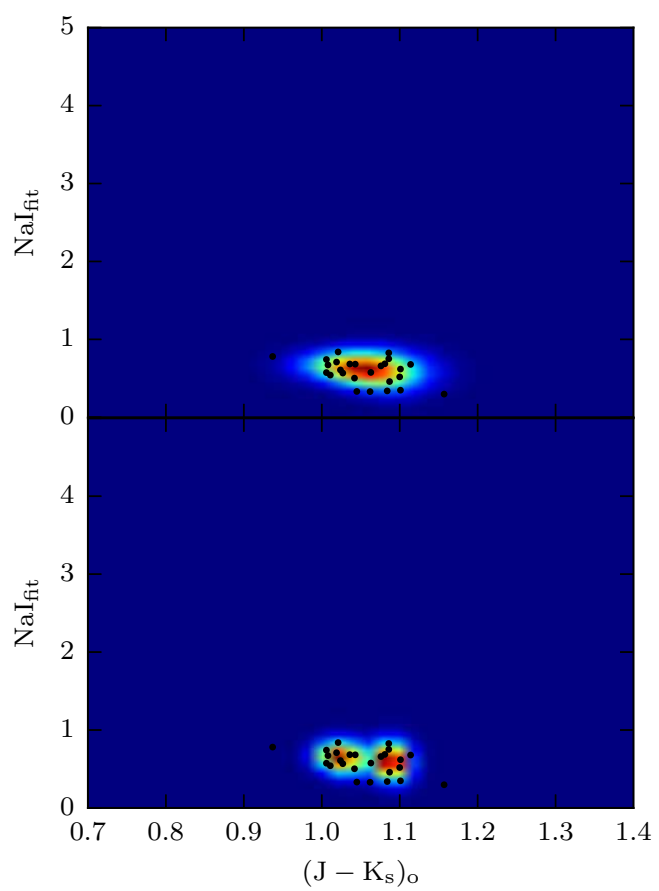

Fig. 3.15. - Same as Figure 3.14, for $\left[\left(J-K_{s}\right)_{o}, W\left(N a I_{f i t}\right)\right]$. 
$2 \mathrm{~d}$ distribution for dwarfs. T.S. 1

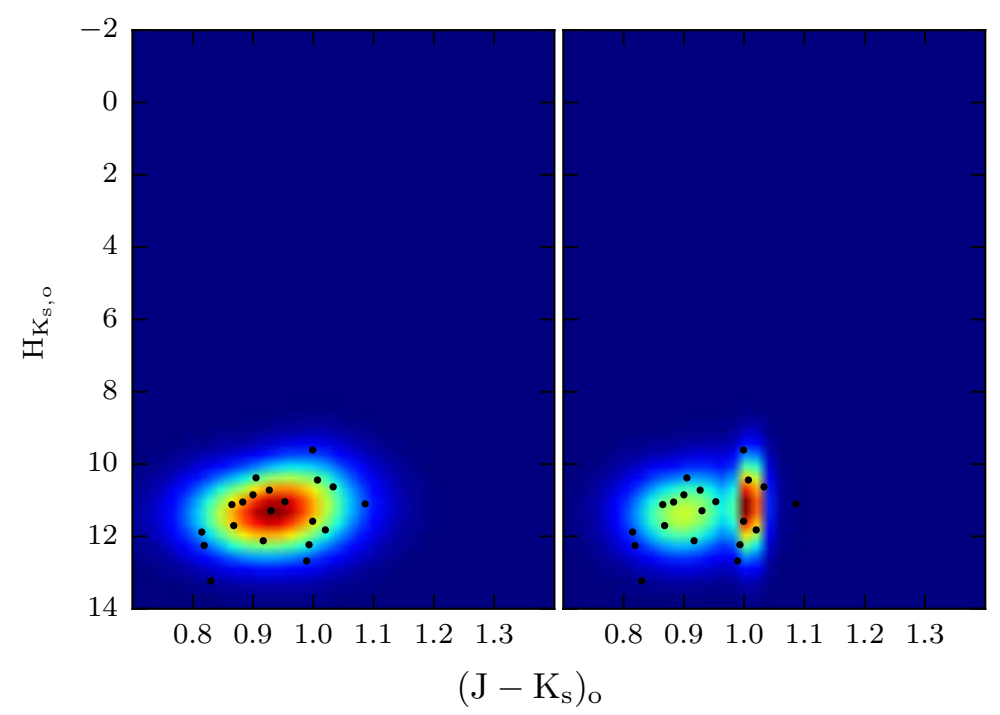

$2 \mathrm{~d}$ distribution for giants. T.S. 1

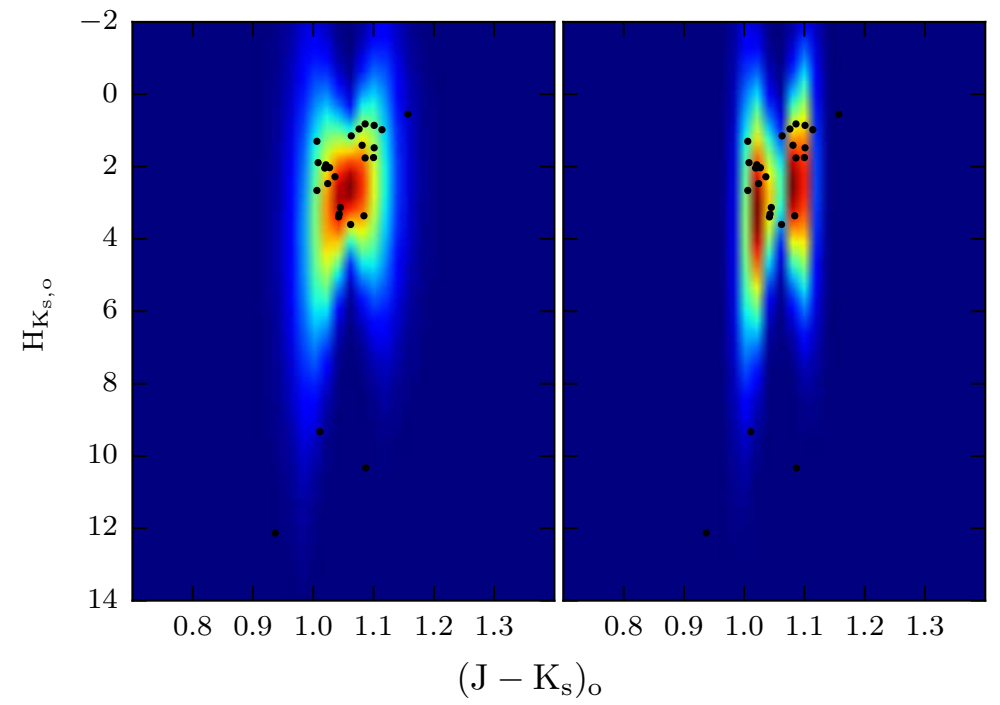

Fig. 3.16. - The 2D PDF and TS1 data for the $\left[\left(J-K_{s}\right)_{o}, H_{K_{s, o}}\right]$ diagnostic. The top panels show the data and results for dwarfs, while the lower panels for giants. The panels in the left column use a single-component Gaussian 1D PDF, whereas panels in the right use a two-component Gaussian. 
2d distribution for dwarfs. T.S. 1

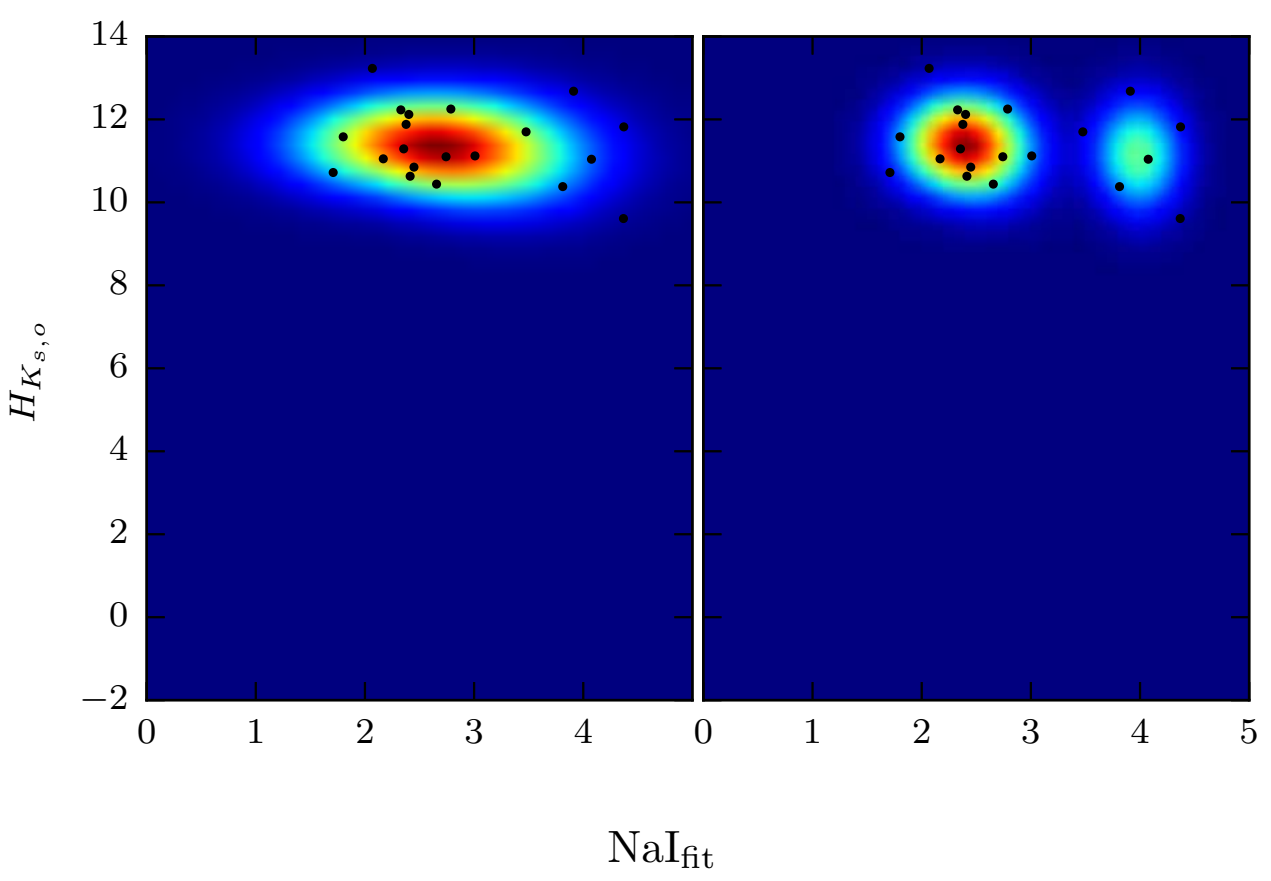

$2 \mathrm{~d}$ distribution for giants. T.S. 1

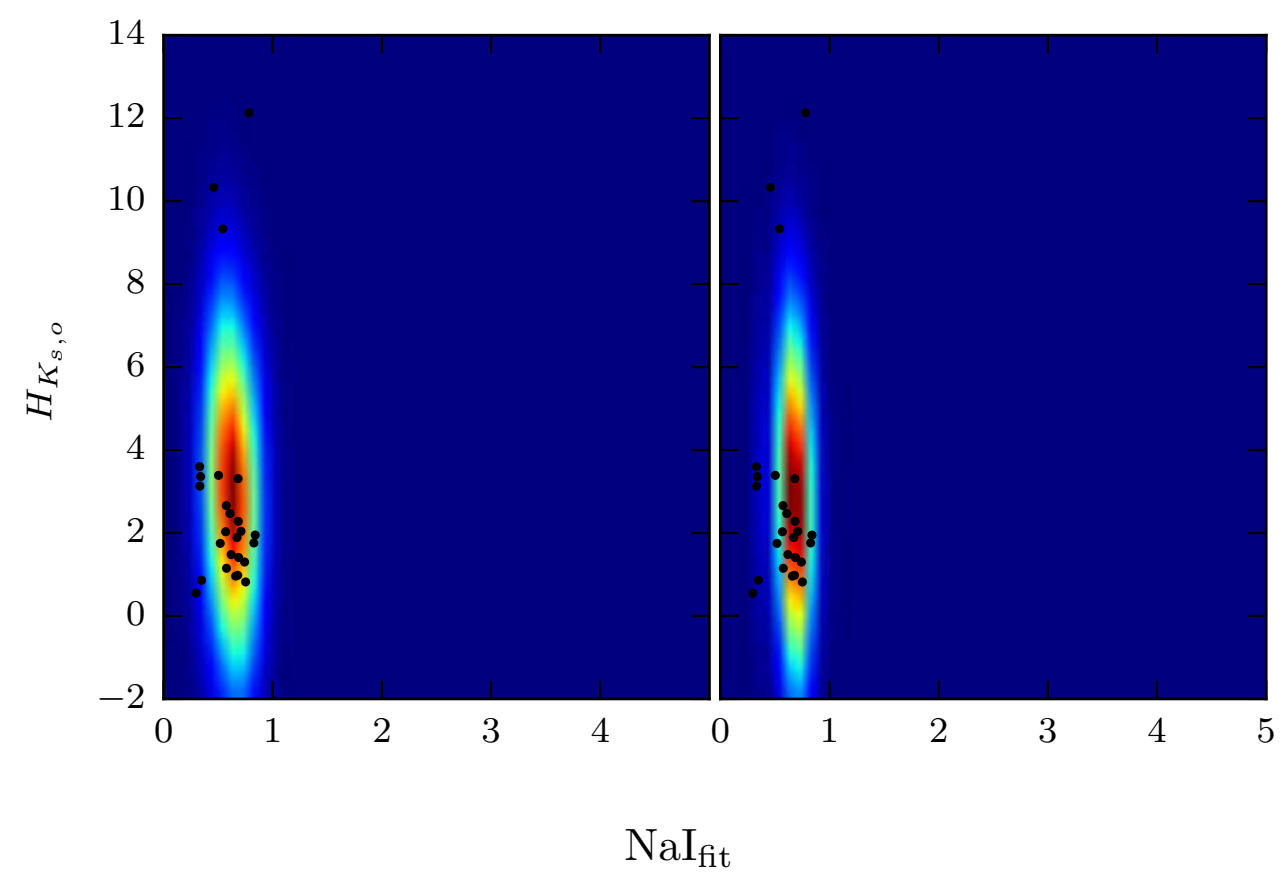

Fig. 3.17. - Same as Figure 3.15, for $\left[W\left(N a I_{f i t}\right), H_{K_{s, o}}\right]$. 
$\mathrm{NaI}_{\text {fit }} 1 \mathrm{~d}$ dist, dwarfs. T.S. 2

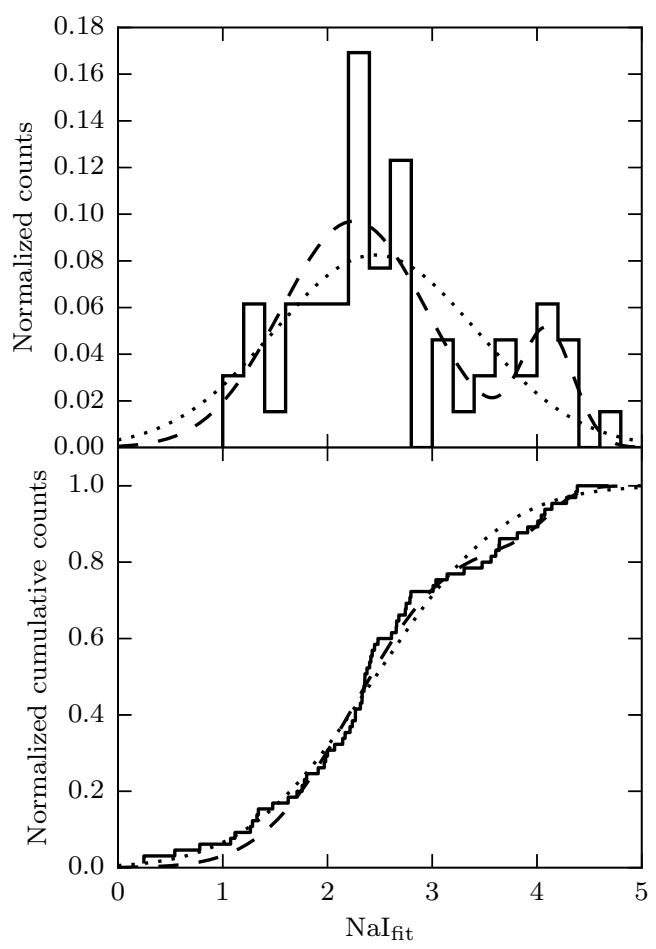

$\mathrm{NaI}_{\mathrm{fit}}$ 1d dist, giants. T.S. 2

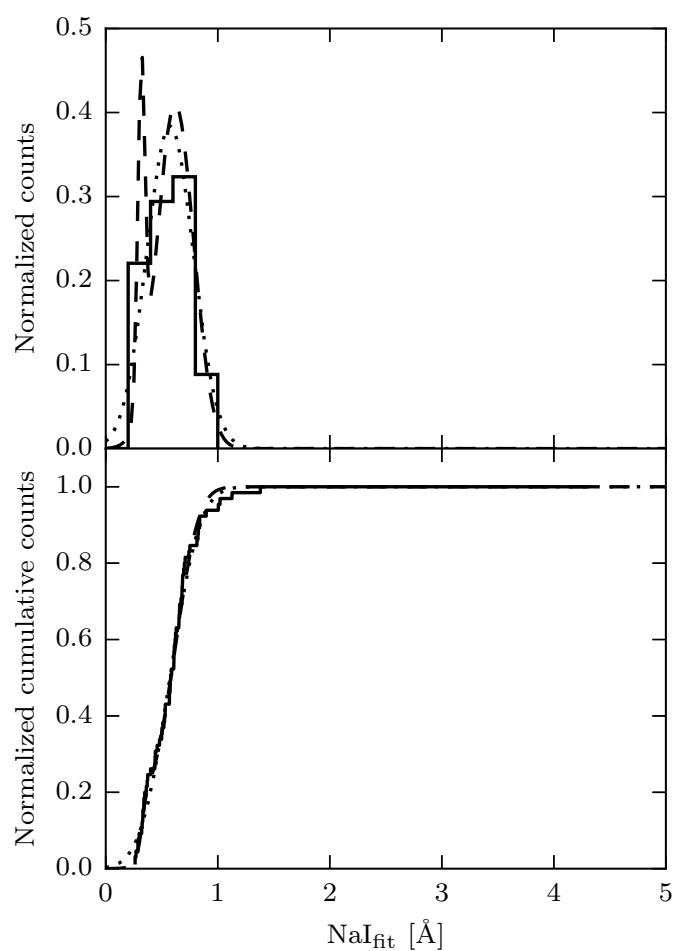

Fig. 3.18. - Same as Figure 3.12, for the Training Set 2 (TS2). 
$\left(\mathrm{J}-\mathrm{K}_{\mathrm{s}}\right)_{\mathrm{o}} 1 \mathrm{~d}$ dist, dwarfs. T.S. 2

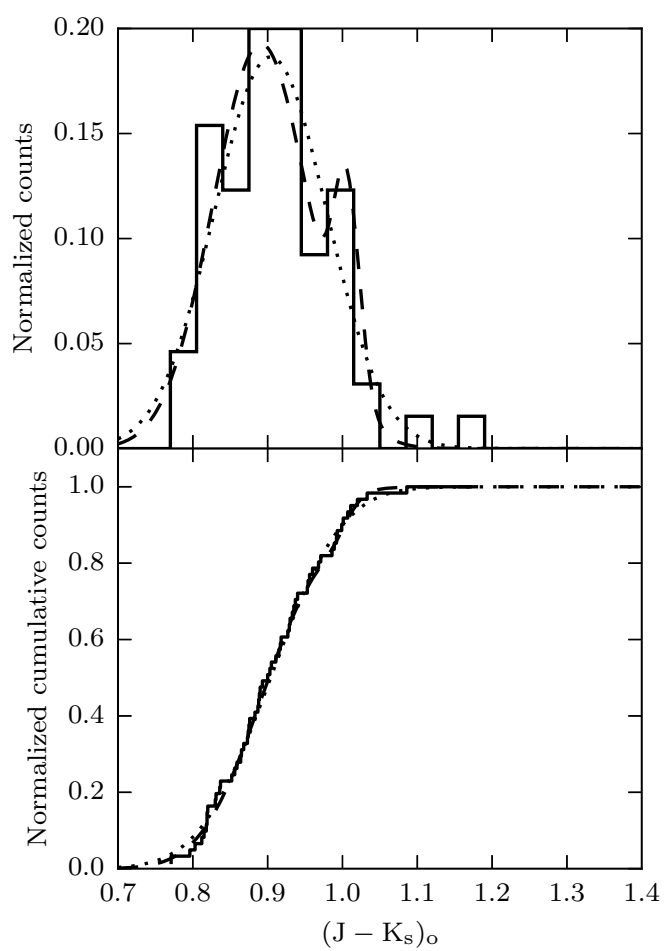

$\left(\mathrm{J}-\mathrm{K}_{\mathrm{s}}\right)_{\mathrm{o}} 1 \mathrm{~d}$ dist, giants. T.S. 2

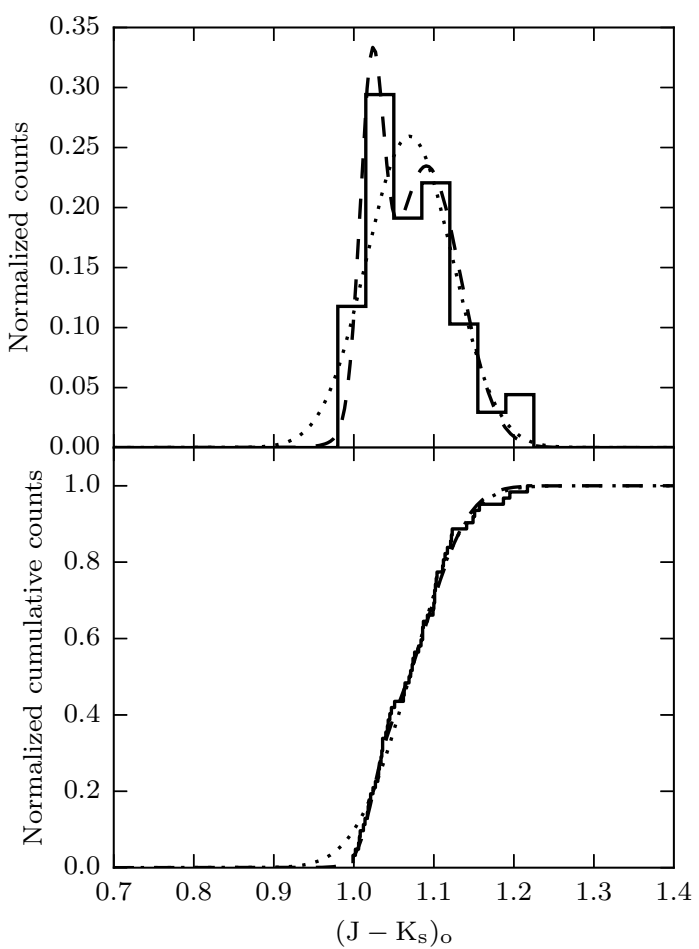

Fig. 3.19.- Same as Figure 3.18, for $\left(J-K_{s}\right)_{o}$. 
2d distribution for dwarfs. T.S. 2

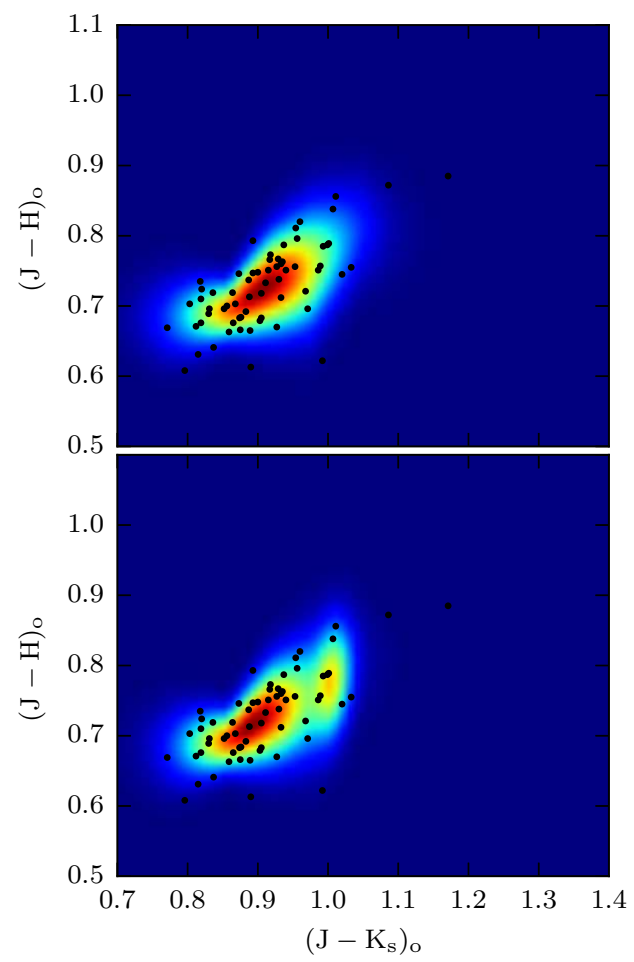

$2 \mathrm{~d}$ distribution for giants. T.S. 2

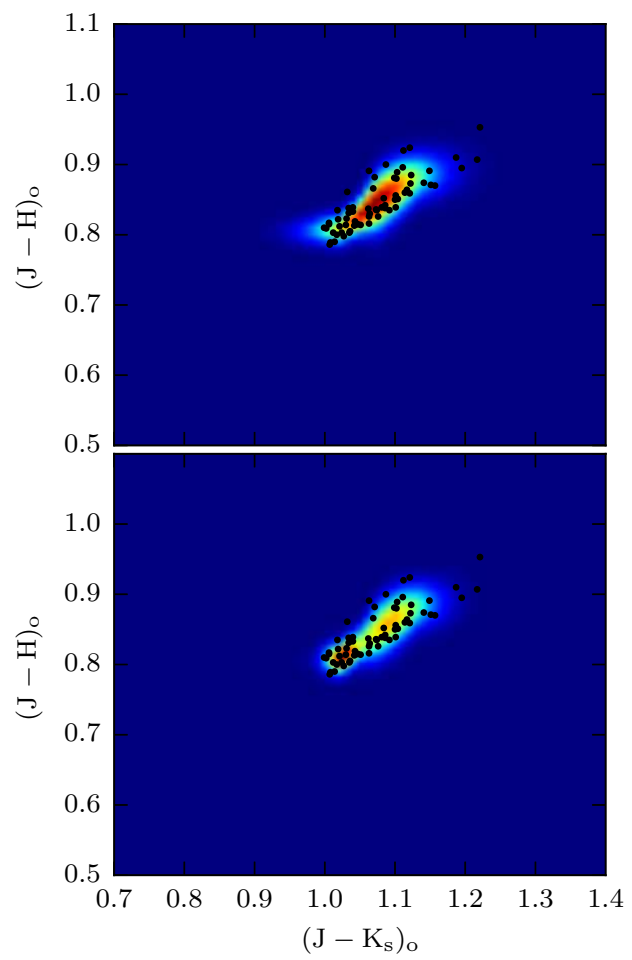

Fig. 3.20.- Same as Figure 3.14, for TS 2. 
2d distribution for dwarfs. T.S. 2

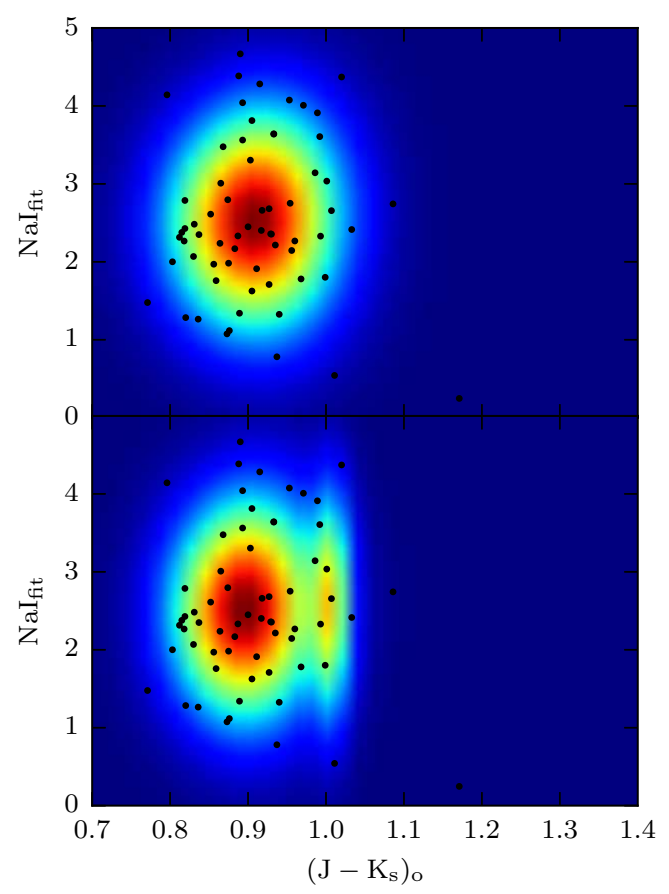

$2 \mathrm{~d}$ distribution for giants. T.S. 2

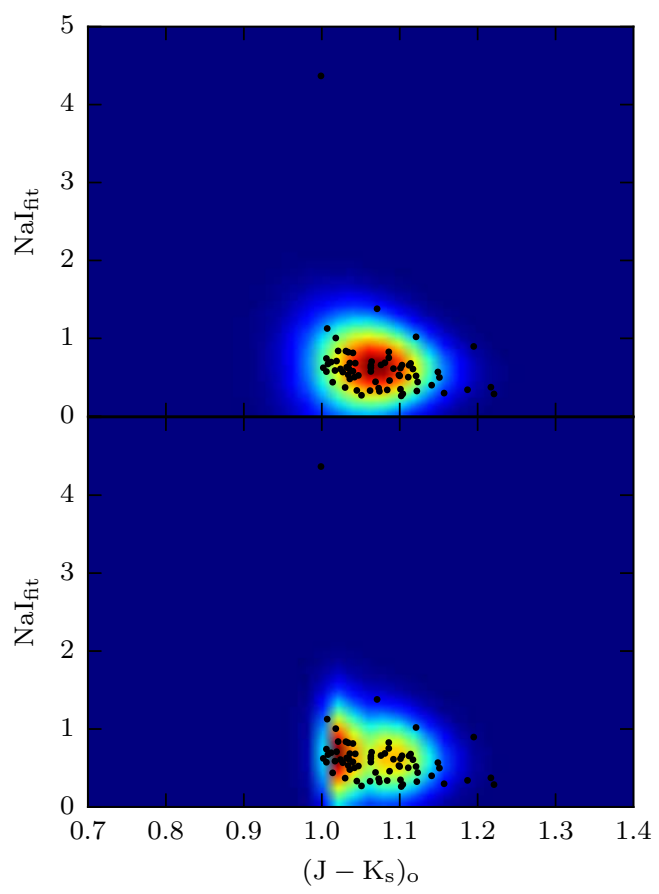

Fig. 3.21. - Same as Figure 3.20, for $\left[\left(J-K_{s}\right)_{o}, W\left(N a I_{f i t}\right)\right]$. 
2d distribution for dwarfs. T.S. 2

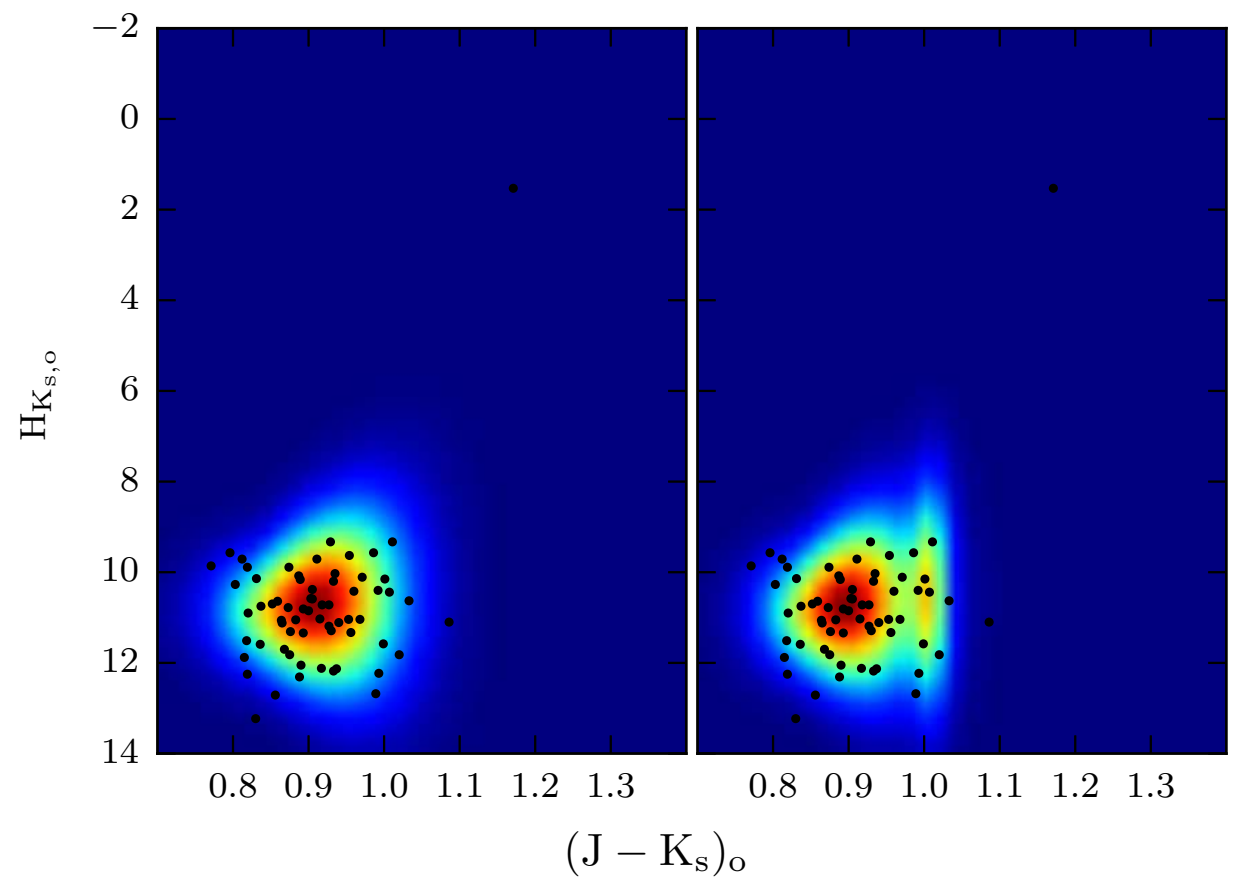

$2 \mathrm{~d}$ distribution for giants. T.S. 2

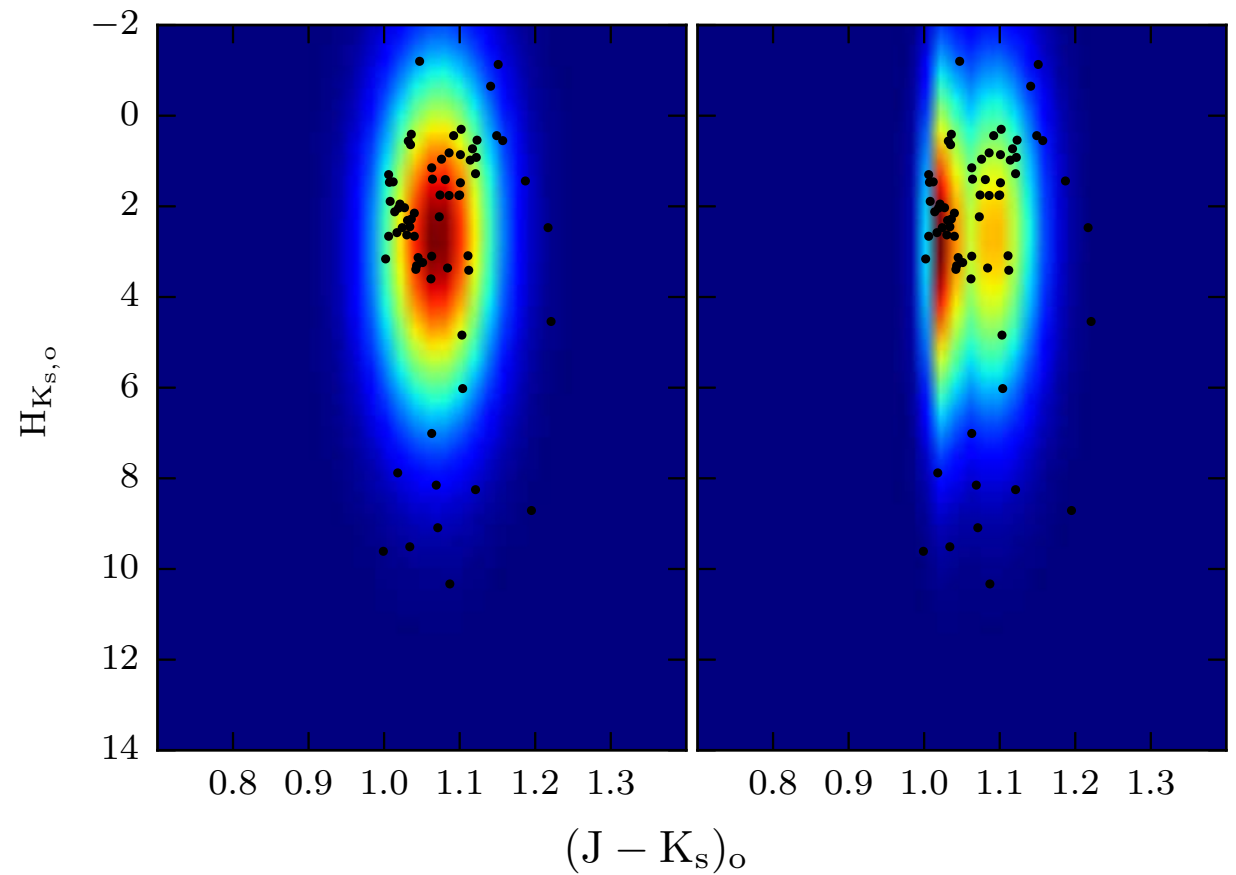

Fig. 3.22.- Same as 3.16, for the the $\left[\left(J-K_{s}\right)_{o}, H_{K_{s, o}}\right]$ diagnostic and TS2. 
2d distribution for dwarfs. T.S. 2

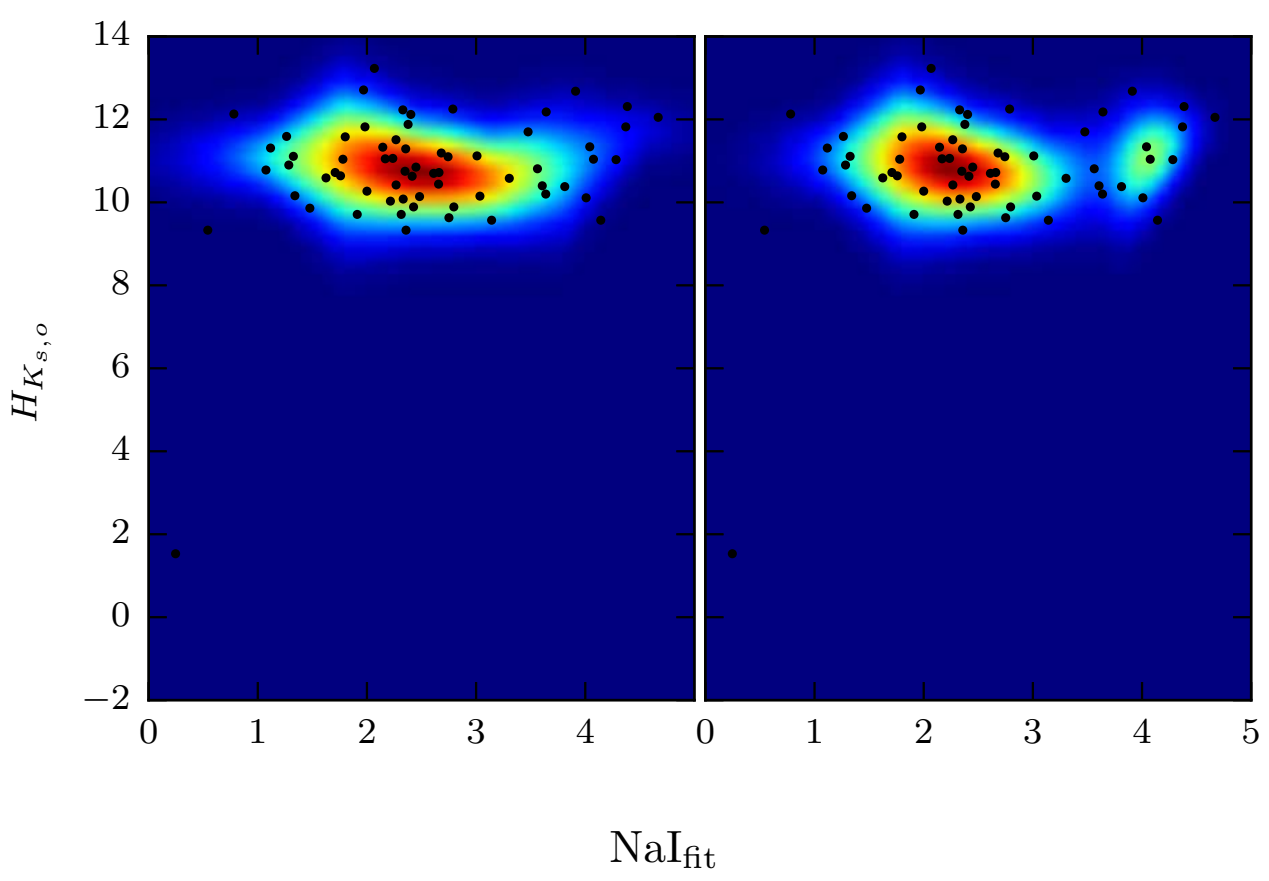

$2 \mathrm{~d}$ distribution for giants. T.S. 2

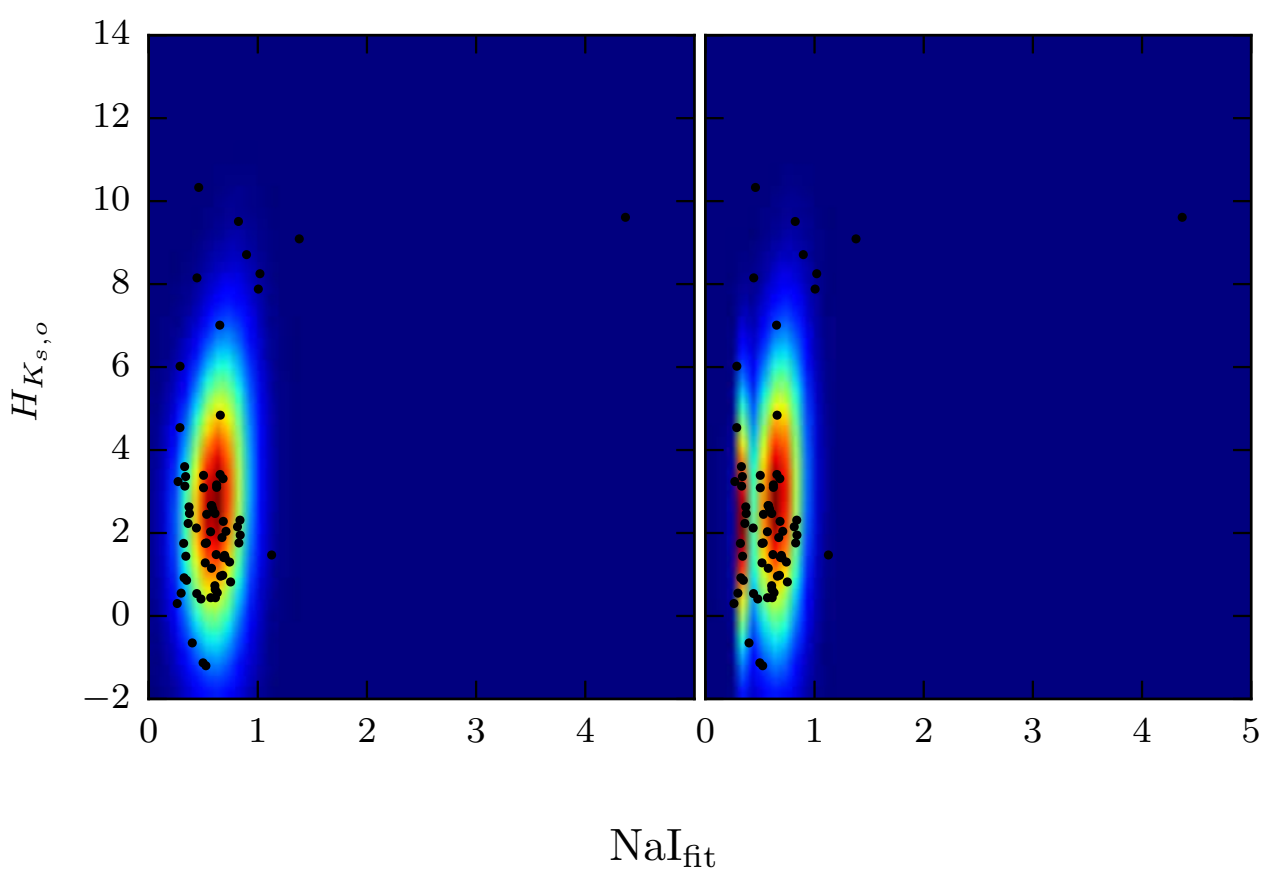

Fig. 3.23. - Same as Figure 3.21, for $\left[W\left(N a I_{f i t}\right), H_{K_{s, o}}\right]$ and TS2. 
7 stars in the sample have no sodium nor good photometry and proper motions. It is probable that these stars were selected from the early 2MASS photometric catalog used by our group, but the updated photometry did not result in a good photometric selection. The evaluated 2D PDFs - calculated for TS2 - and the resulting giants/dwarfs for the whole sample of stars is presented in Figures 3.24 and 3.25. We present the clean sample of giants at the beginning of Section 3.3 .

\subsubsection{The Ca II Triplet Equivalent Width and Metallicity Estimation}

In Chapter 2, we developed a method to estimate metallicities using the near-IR Ca I triplet (CaT hereafter) strength for isolated stars using isochrones and 2MASS colors. Section 2.2 specifically introduces the definition of the "Equivalent Width" (W, Eq. 2.1) as an estimator of the strength of each line in the triplet, the line fitting equation (Eq. 2.2), line bandpasses (Table 2.1) and data fitting procedure and code used to perform the fit and measure $W$ for each triplet line from spectra. In this section, we present the $\mathrm{CaT}$ measurements for the spectroscopic dataset, and then calculate metallicities.

We use a Python program that prepares the spectra and then performs the $W$ measurement of the triplet. First, the code trims the spectra into two separate pieces, which provides better results for the normalization. One part is trimmed to $[8,400<\lambda<8,610]$ and contains the continuum and line bandpasses for the first and second lines of the triplet. The other section is trimmed to $[8,550<\lambda<8,730]$ and contains the continuum and line bandpasses of the third line. Then the computer program normalizes each section using IRAF's continuum task in non-interactive mode. Trimming into short spectrum chunks allows us to fit a 2nd order polynomial — 3 rd 
$2 \mathrm{~d}$ distribution for all, using T.S. 2

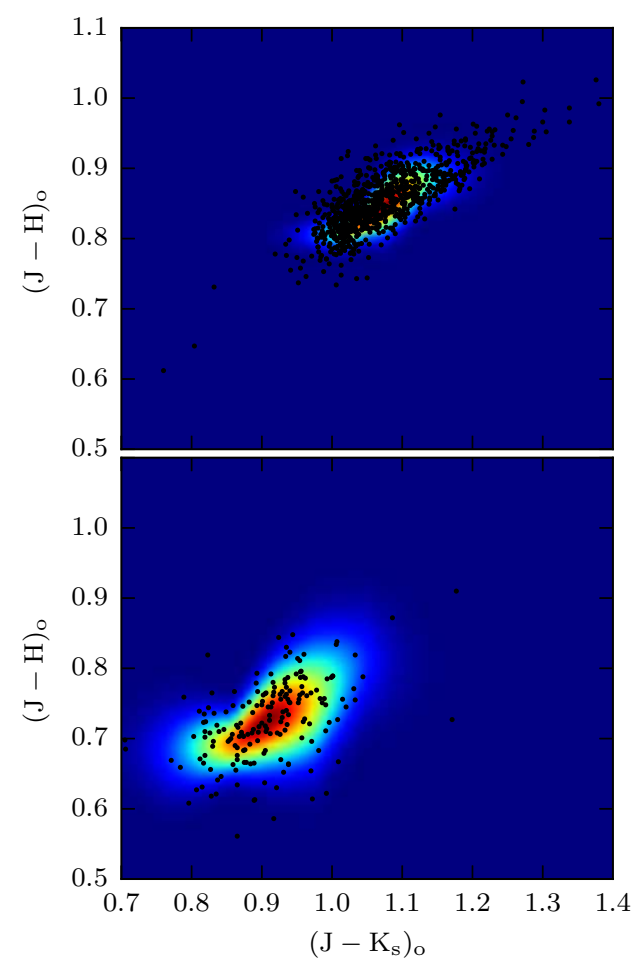

$2 \mathrm{~d}$ distribution for all, using T.S. 2

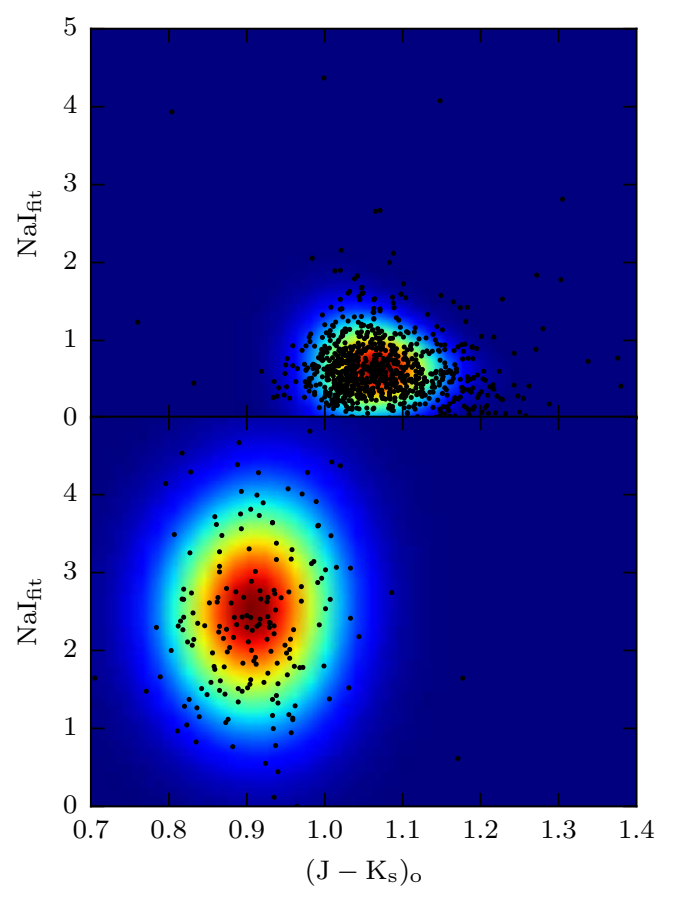

Fig. 3.24. - The whole sample of stars, selected as giants (top panels) and dwarfs (bottom panels), evaluated using TS2 PDFs. The panels on the left show the $[(J-$ $\left.\left.K_{s}\right)_{o},(J-H)_{o}\right]$ diagnostic, while the $\left[\left(J-K_{s}\right)_{o}, H_{K_{s, o}}\right]$ diagnostic is on the right side. 
2d distribution for all, using T.S. 2

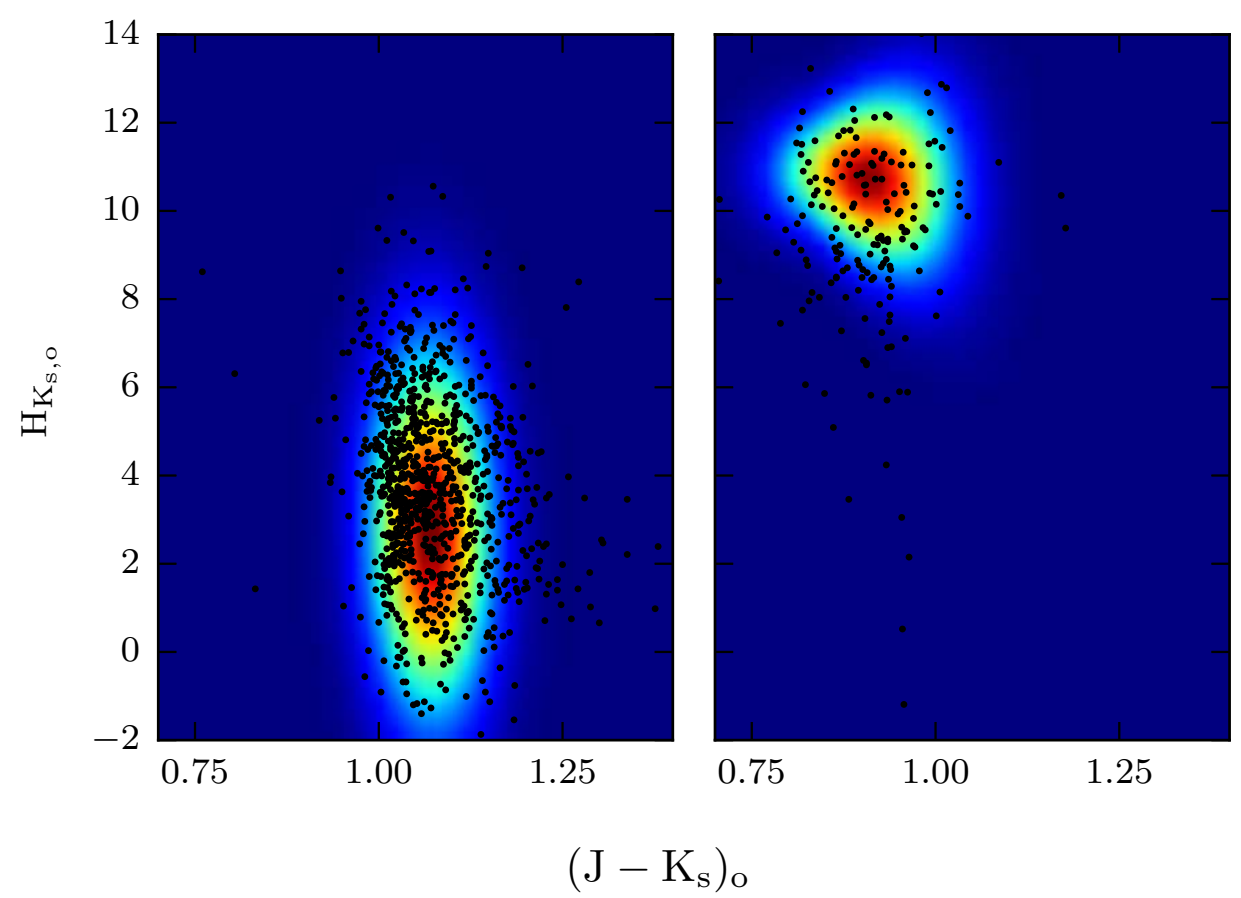

$2 \mathrm{~d}$ distribution for giants. T.S. 2

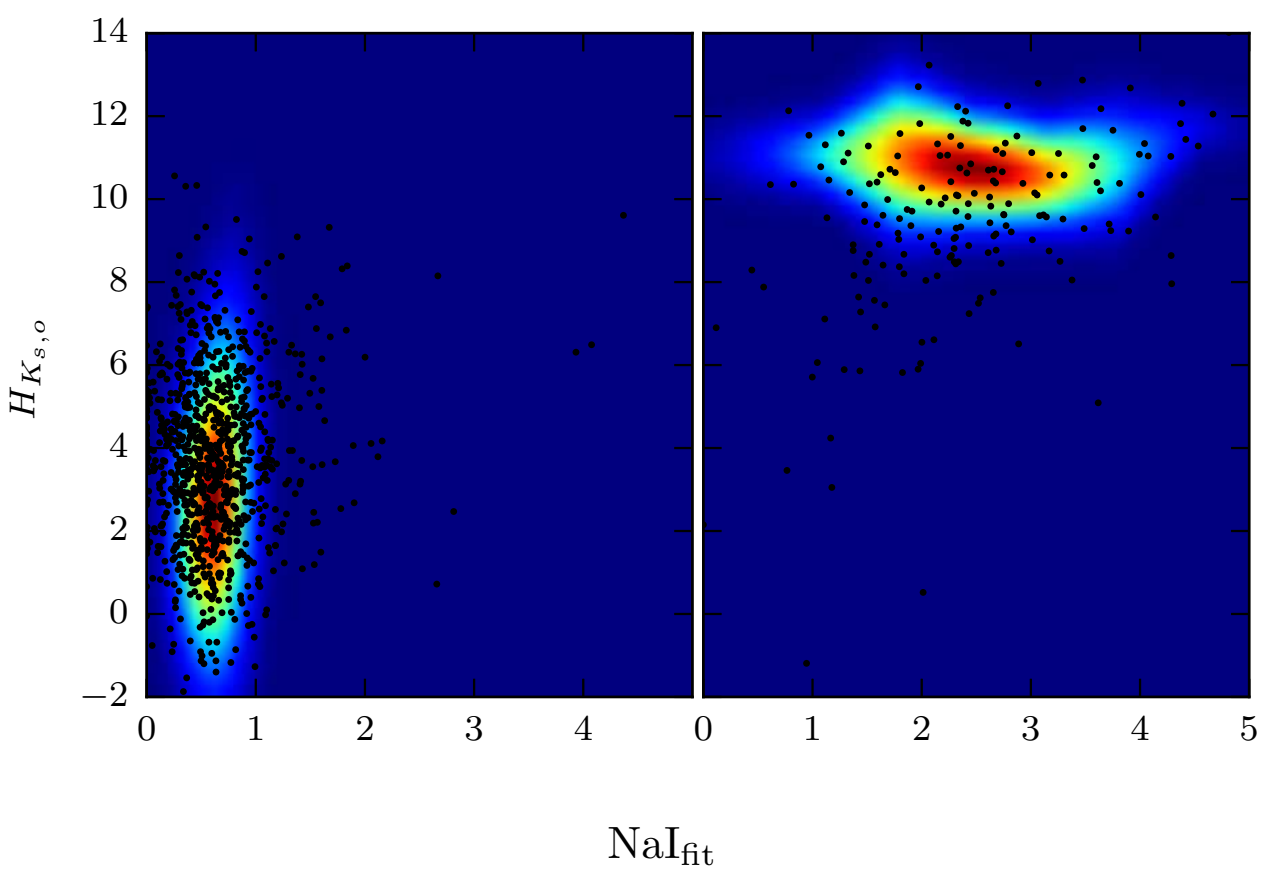

Fig. 3.25. - Same as Figure 3.24, for the $\left[\left(J-K_{s}\right)_{o}, H_{K_{s, o}}\right]$ diagnostic on the left, and the $\left[\left(J-K_{s}\right)_{o}, H_{K_{s, o}}\right]$ diagnostic on the right. 
order in IRAF's nomenclature - to describe the continuum well. Using longer pieces of spectra could require higher polynomial orders, which may result in oscillations of the fit around the actual continuum. The continuum normalization only uses portions of the spectrum free of spectral lines. The low- and high-rejection parameters of continuum were set to 1.5 and $3.5 \sigma$, respectively. This selection includes more points below the continuum than above, affectively setting the continuum level higher than using equal sigmas. We expect that this choice will make the continuum placement less prone to spectral lines that otherwise would falsely lower the continuum value.

Next, the code applies the steps given in Section 2.2 for each line in the triplet. We measure two values of $W$ via Equation 2.1, one where we fit a straight line to the continuum bandpasses (Table 2.1), and another one where the continuum is fixed to 1.0. We estimate the signal-to-noise ratio (SNR) for each line as the square-root of median number of photo-electrons within the continuum bandpass. The uncertainty in $W$ for each line is calculated as:

$$
\sigma(W)=\frac{\sqrt{n_{p i x}} * \Delta_{p i x}}{S N R}
$$

where $n_{p i x}$ and $\Delta_{p i x}$ are the number of pixels and pixel width (in Angstroms) within the line bandpass. The dataset has 1,146 stars with CaT measurements, where 180 stars have SNR greater than 50. Figure 3.26 shows the distribution of the SNR of the spectra, while Figure 3.27 shows the histograms $\sigma(W)$ and $W$ versus $\sigma(W)$ for the three Ca lines.

We calculate metallicities using the isochrone method described in 2.3.2, where we solve for metallicity iteratively. The input parameters are the $\left(J-K_{s}\right)_{o}$ color, the measured CaT $W$ and the respective uncertainties. Following the definition of Warren \& Cole (2009), we calculate $W$ as the unweighted sum of the equivalent 


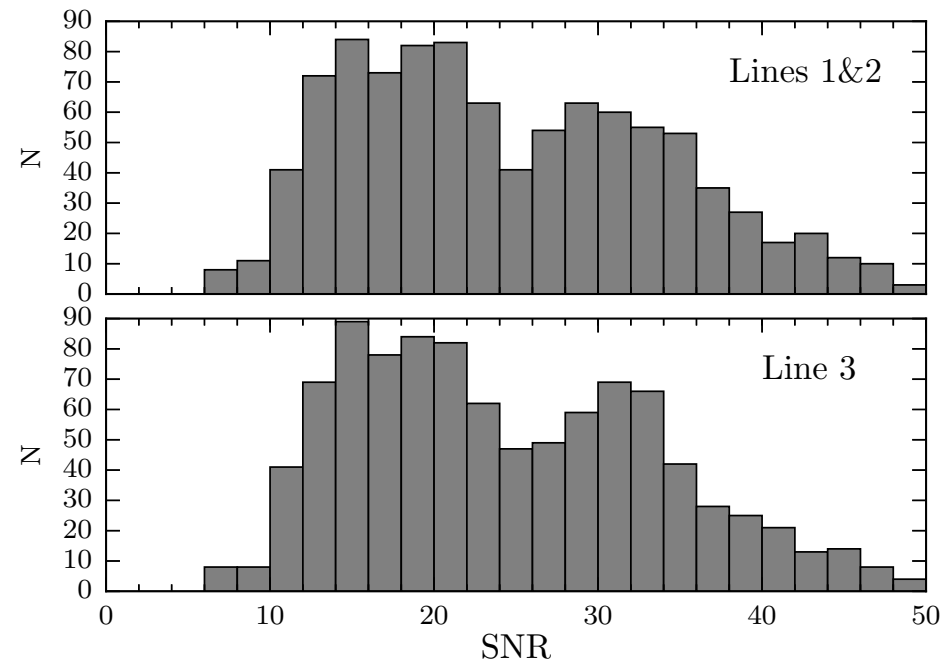

Fig. 3.26. - Histograms of the signal-to-noise ratio of the science spectra, for the spectral regions containing the first $(\lambda 8498)$ and second lines $(\lambda 8542)$ of the triplet (top panel), and the third line $(\lambda 8662)$ of the triplet (bottom panel).
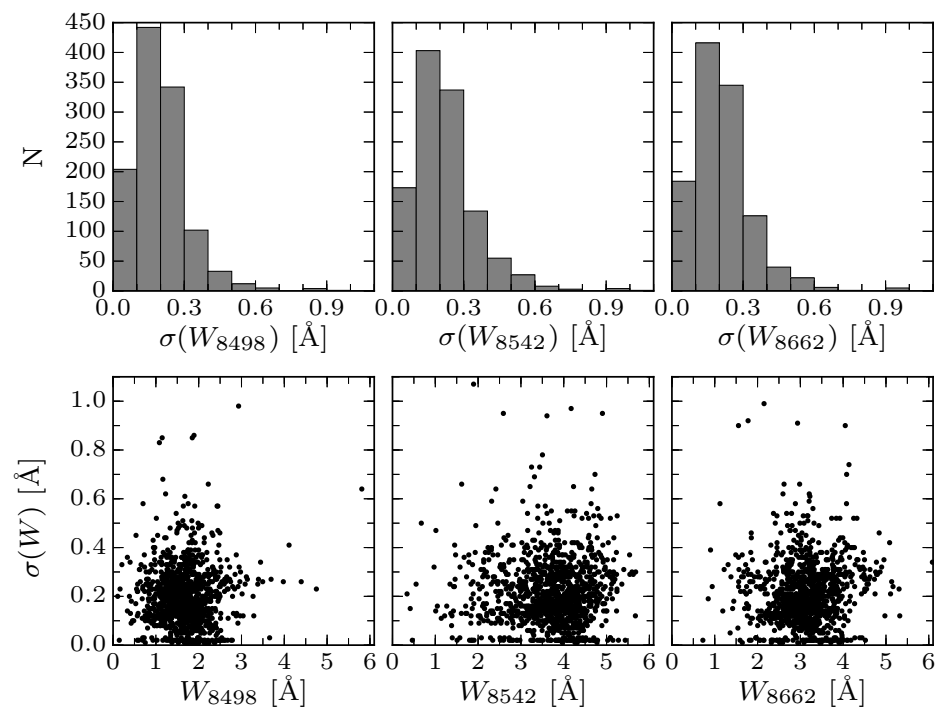

Fig. 3.27. - CaT equivalent widths $W$ and uncertainties $\sigma(W)$ for the science spectra. Top panels: Histograms of the CaT equivalent width uncertainty $\sigma(W)$. Bottom panels: Equivalent width $W$ versus uncertainty $\sigma(W)$. The top 
widths of the three lines. The uncertainties in color and $W$ are calculated as the square root of the sum in quadrature of the respective uncertainties. We use a singleage isochrone to derive metallicities, because we have shown that metallicities derived through isochone-based CaT calibrations are independent of isochrone age. We derive the uncertainty in metallicities by calculating the mean in difference in resultant metallicity between $W$ and $W \pm \sigma(W)$ - for the star's $\left(J-K_{s}\right)_{o}$-, and between $\left(J-K_{s}\right)_{o}$ and $\left(J-K_{s}\right)_{o} \pm \sigma\left(\left(J-K_{s}\right)_{o}\right)$ - for the star's $W$ - The reported uncertainty is calculated as the square root of the sum in quadrature of these two uncertainty estimations.

We present the results of the metallicity measurements in the following section.

\subsection{Analysis and Results}

\subsubsection{The tidal tails of Sagittarius traced by M-giants}

From the sample of giants obtained in Section 3.2.4, we begin by identifying the likely members of the leading and trailing arms of Sgr. The giants are selected from the results of the overall giant/dwarf likelihood $\left\langle L_{i}\right\rangle$, and shown in Figures 3.28 and 3.29. The tidal disruption of a satellite like Sgr forms coherent structures (tidal tails) in several spaces, and we exploit these to trace the tails. For instance, Majewski et al. (2003) showed that Sgr core and tidal debris lie close to a great circle defined by the core orbit, as seen in 2MASS M-giants. Sections of these coherent structures have also been observed using Main Sequence Turn Off stars selected from the SDSS (Belokurov et al. 2006; Koposov et al. 2012) in the SDSS footprint. We constrain the search area using the known position of the debris on the sky. We therefore keep stars close to Sgr's coordinate system equator by requiring $\left[-30^{\circ}<B_{\odot}<30^{\circ}\right]$ for the candidate 
selection of Sgr stars. This margin is broader than the observed distributions of stars in Sgr, but we use this range just as an initial selection. From the 1,257 giant stars in the sample, 890 pass this cut.

In addition, stars along the tidal tails form coherent features in the $\left(\Lambda_{\odot}, \mathrm{V}_{\mathrm{GSR}}\right)$ and $\left(\Lambda_{\odot}\right.$, distance) planes, as the debris approximately delineate the orbit of the progenitor (Johnston 1998; Johnston et al. 2008). Because we do not know the distance to the stars in the sample, we use magnitudes as a proxy of distance to explore the $\left(\Lambda_{\odot}\right.$, distance) plane. However, the $K_{s, o}$ magnitude of RGB stars increases with $(J-$ $\left.K_{s}\right)_{o}$ color. Therefore, it is necessary to remove this variation in $K_{s, o}$ first. We use the slope of the absolute magnitude-color linear relation of Majewski et al. (2003) — derived from stars in Sgr's core - to define a "normalized" $K_{n}$ magnitude as the color-corrected $K_{s, o}$ magnitude that a RGB star would have at $\left(J-K_{s}\right)_{o}=1$. Then, the normalized magnitude of a star is:

$$
\left.K_{n}=8.650\left[\left(J-K_{s}\right)_{o}\right)-1\right]+K_{s}
$$

We present a comparison between $K_{s, o}$ and $K_{n}$ in Figure 3.30.

To trace the tails, we look for coherent structures in the three planes discussed previously along the Sgr tails. We implement an interactive program in Python that allows us to cross-select stars in the three parameter spaces simultaneously. We begin selecting stars in the $\left(\Lambda_{\odot}, V_{G S R}\right)$ plane along the known portions of both tails. The selected stars begin to form a clear locus around the equator of Sgr's coordinate system in the $\left(\Lambda_{\odot}, B_{\odot}\right)$ plane, which is expected by definition of the coordinate system. In addition, the stars selected also define a clear locus in the $\left(\Lambda_{\odot}, K_{n}\right)$ plane. We then extend the selection from higher to lower $\Lambda_{\odot}$ along the leading arm, and lower to higher $\Lambda_{\odot}$ in the trailing arm. Figures 3.31 and 3.32 show the three spaces used 


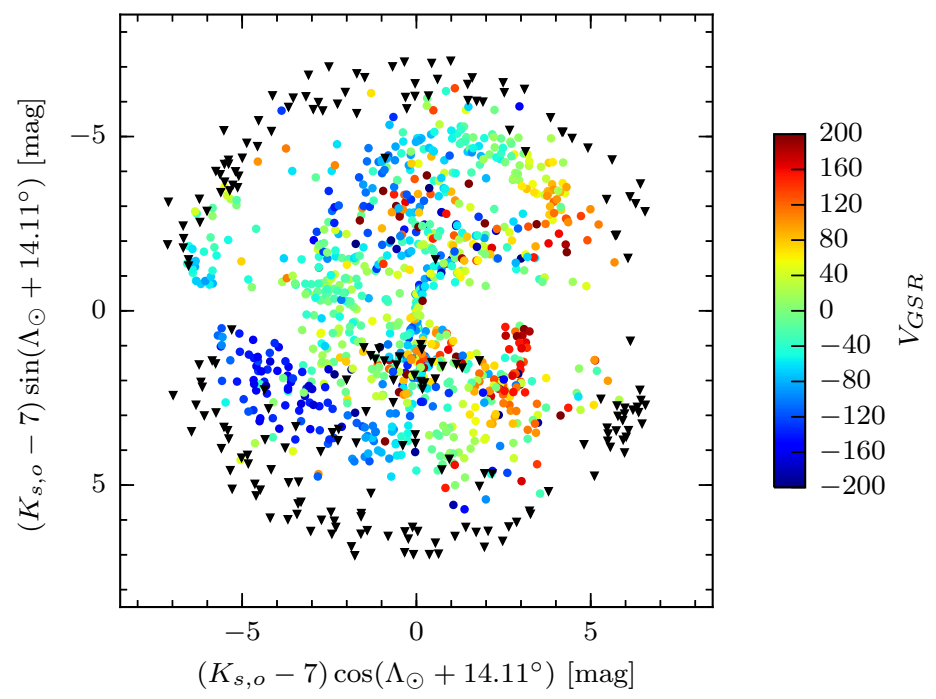

(a) Same as Figure 3.5a, but illustrating the results for the giant/dwarf separation. Likely dwarfs - i.e., $\left\langle L_{i}\right\rangle<0$ - are marked with black triangles.

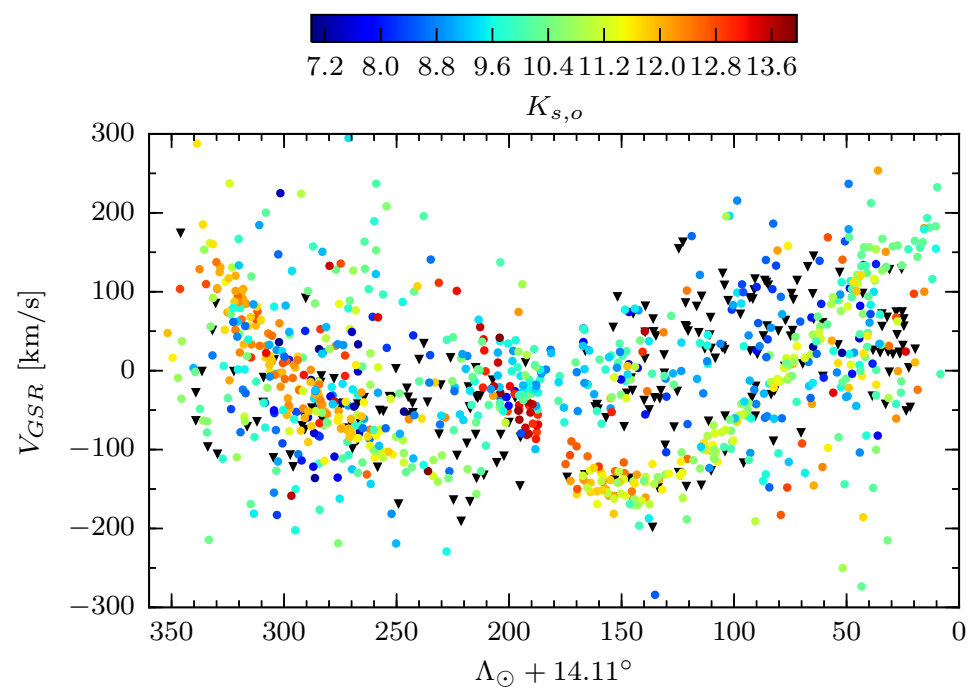

(b) Same as Figure 3.5b, but for the results of the giant/dwarf selection. Likely dwarf stars are marked with black triangles.

Fig. 3.28.- Results for the giant/dwarf separation from the overall likelihood $\left\langle L_{i}\right\rangle$ (Equation 3.9).

for tracing stars likely belonging to the trailing and leading arms, respectively. The figures include the whole sample of giant stars and highlight the stars selected as 
likely Sgr members. The selection results in 206 and 146 stars for the trailing and leading arms, respectively.

\subsubsection{Comparison to the SDSS View and the Law \& Majew- ski (2010) Model}

We compare the on-sky position of the stars in the sample against the known on-sky position of the stream in Figure 3.33. Using star counts of upper main sequence and turnoff stars from SDSS data (DR5), Belokurov et al. (2006) found that the northern/leading arm is bifurcated. They identified a high-density (A) and a low-

density (B) branch. These branches run more or less in parallel passing through the North Galactic Cap, with branch A running at lower declinations than branch B i.e., branch A has higher Sgr $B$ latitude than the branch B. Similarly, Koposov et al. (2012) identified a low-density branch running parallel to the main high-density Sgr southern/trailing arm in SDSS DR8 data with a similar strategy of Belokurov et al. (2006). Koposov et al. (2012) named the high/low-density stream as the bright/faint branch — we will use both designations for the leading and trailing arms. We place our sample on top of the SDSS view of Koposov et al. (2012) in Figure 3.33.

In general, we find that the sky position of the Sgr debris detected in M-giants agree with the fields detected in SDSS and Pan-STARRS1, except that the M-giants distribution seem to be slightly broader on the sky perpendicular to plane of the debris. For the leading arm, we detect stars along both the bright and faint branches of the stream. We do not detect stars with angular separation from the core larger the fields described Belokurov et al. (2006), as measured in $\Lambda_{\odot}$. An intriguing feature that we observe is a "deviation" of the M-giants between $\Lambda_{\odot}=\left[225^{\circ}, 200^{\circ}\right]$ towards higher $\mathrm{B}_{\odot}$ compared to the locus delineated by SDSS main sequence tracers (Belokurov et al. 
2006). Figure 3.34 shows the position of these stars on the sky with more precision, along with GSR velocities and $K_{n}$ magnitudes. Besides the group of seven stars that lie within the known fields of the bright branch, there are four stars with $B_{\odot}>15^{\circ}$. We conjecture that these 4 stars are part of Sgr leading arm because (1) these stars follow the same sequence of GSR velocity as those at similar Sgr longitudes within the known A branch, (2) the stars follow the same sequence in $K_{n}$ magnitude as the A-branch stars, and (3) there is no trend in $V_{G S R}$ nor $K_{n}$ magnitudes with respect to Sgr latitude for all the stars in this region. Therefore, we expect that these debris belong to the same progenitor based on the similar dynamical characteristics.

For the trailing arm, we find a good agreement with the position of the known debris described by Koposov et al. (2012) and Slater et al. (2013). We detect stars along the bright and faint structures. In addition, we detect trailing arm stars running parallel of the trailing arm but at lower declinations.

We compare the data against the Law \& Majewski (2010, LM10 hereafter) Nbody model of Sgr disruption. The LM10 model is a full N-body simulation in a static 3-component potential of $10^{5}$ particles, and was designed to produce the best match to the data available at that time. To date, this is the best model available in the literature in terms of matching the on-sky position, GSR velocity and distance of the tidal debris of Sgr.

We begin our comparison by first focusing on the deviation of the M-giants of the leading arm. Figure 3.35 shows the sample and the LM10 model for the leading arm. From the figure, it is clear that the first wrap of the model predicts the position on sky of the M-giants for $\left[320>\Lambda_{\odot}>230\right]$ with great success. However, stars at smaller longitudes seem to deviate to higher $B_{\odot}$. Particularly, the position of the stars in the deviation match the $P_{c o l}=5$ debris of the second wrap better, which were striped 
around 4.6 Gyr ago according to the model. This presents two problems. First, the number of model particles in the second wrap is very low compared to the number of particles in the first wrap. Because the density of N-body particles represent the probability of finding debris at some particular volume in 6D phase space, this implies that the probability of finding these debris in the spectroscopic sample is minuscule compared to stars in the first wrap. Second, the GSR velocity of second wrap in LM10 is more than $200 \mathrm{~km} / \mathrm{s}$ higher at this longitude. Therefore, it is more likely that the M-giants belong to first wrap. In Figure 3.36, we plot the stars around the deviation along with the model particles of the first wrap with Pcol 2,3,4 and 5. From visual inspection, the most likely origin of the debris around $\left[195^{\circ}<\Lambda_{\odot}<205^{\circ}\right]$ is the $\mathrm{Pcol}=5$ debris. We obtain a probability estimation of debris stripping age by counting the number of model particles within this longitude range. Debris with Pcol 3,4 and 5 have $29 \%, 21 \%$, and $31 \%$ of the counts respectively, while Pcol values of 2, 6 and 7 contain the remaining 19\%. However, these fractions vary with longitude. For $B_{\odot}>10^{\circ}$ and $15^{\circ}$, the relative fractions for Pcol equal to $(3,4,5)$ are $(14,23$, $46) \%$ and $(0,29,45) \%$, respectively. Therefore, it is more likely that the stars in the deviation correspond to $\mathrm{Pcol}=5$ debris.

Next, we compare the model and the trailing arm data in Figure 3.38. The model depicts the position on sky and the GSR velocity of the stars with $\left[0^{\circ}<\Lambda_{\odot}<140^{\circ}\right]$ well. This is expected because LM10 included trailing arm data within this longitude range to create the model ${ }^{5}$. On the contrary, the position and GSR velocity predicted by the model drifts away from the locus defined by the data as longitude increases for $\Lambda_{\odot}>140^{\circ}$. Nevertheless, we still can use the model to estimate the model age of the debris that is in disagreement with the data. As for the leading arm, we calculate the

\footnotetext{
${ }^{5}$ Radial velocities for trailing arm used by LM10 were published in Majewski et al. (2004) and Law et al. (2005). We have re-reduced and measured the raw spectra of both publications in this chapter.
} 
fraction of debris in the first wrap of the trailing arm within $\left[170^{\circ}<\Lambda_{\odot}<200^{\circ}\right]$, for different Pcol values. The relative fractions for debris with $\mathrm{Pcol}=(3,4,5)$ are $(31 \%$, $41 \%, 25 \%)$. If we consider that the stars in our sample in the longitude interval as having $B>-5^{\circ}$, we find $\mathrm{Pcol}=(3,4,5)$ equal to $(37 \%, 40 \%, 17 \%)$. We present the trailing arm data and the Pcol values for this particular region in Figure 3.37.

We find that the model fails to reproduce features that were likely stripped from the core at pericenter passages older than Pcol equal to 4 or 5. According to LM10, this correspond to 3.6-4.6 Gyr ago. We explore orbital precession as a possible cause of the disagreement between model and data. 


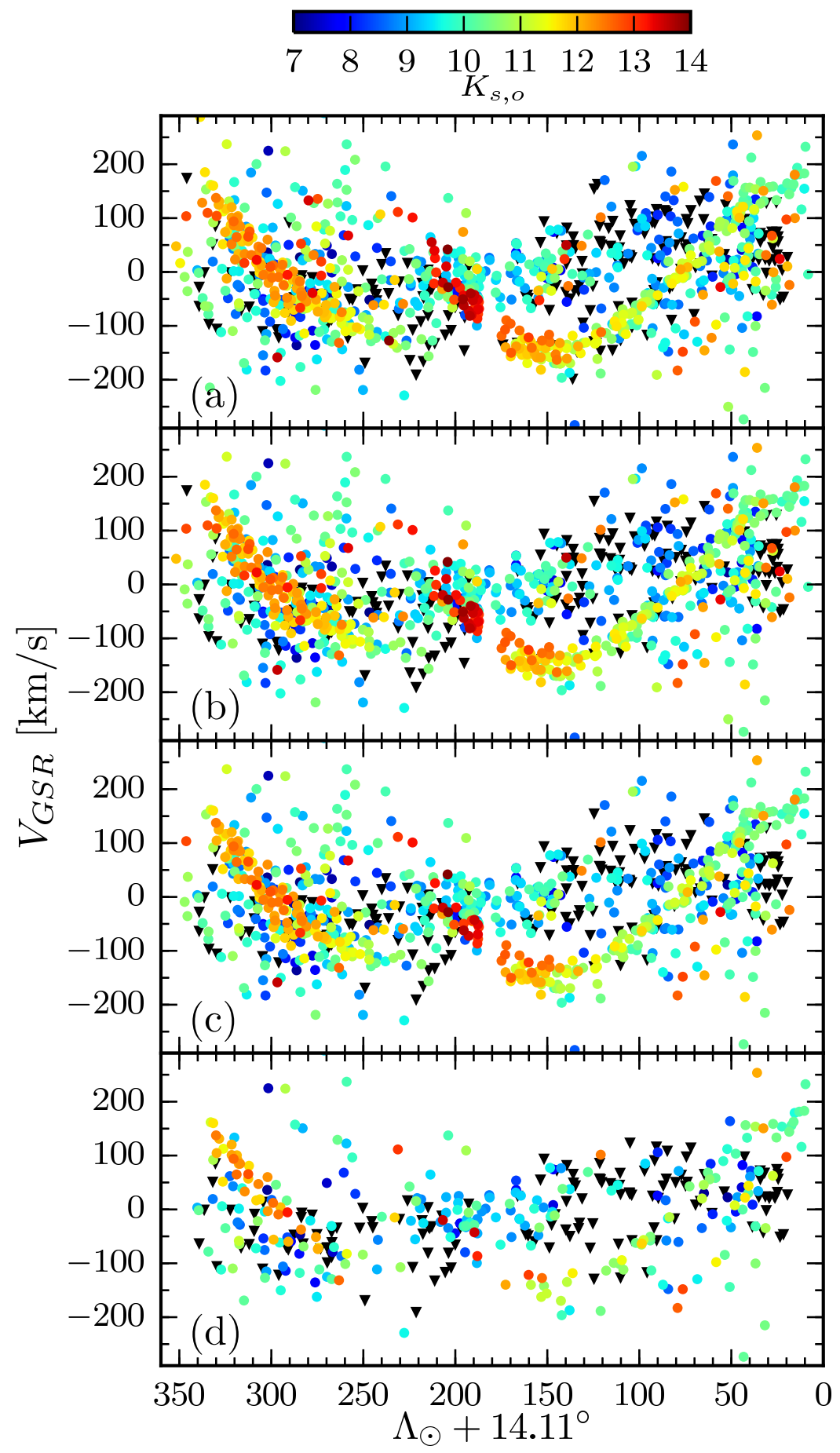

Fig. 3.29.- The $\Lambda_{\odot}$ versus GSR radial velocity plane for different levels in the overall giant/dwarf likelihood. Stars with overall likelihood $\left\langle L_{i}\right\rangle>0$ (Equation 3.9) are considered giants and are color-coded by $K_{s, o}$ magnitude, while stars with $\left\langle L_{i}\right\rangle<0$ are considered dwarfs and presented as black triangles. Panels (a) to (d) show increasingly stronger likelihood thresholds for the stars selected as giants/dwarfs. We have set the $\left\langle L_{i}\right\rangle<0$ threshold at $0 ., \pm 1, \pm 2$ and \pm 3 the, for panels (a), (b), (c) and (d), respectively. For these thresholds, the number of giants (dwarfs) is 890 (209), 842 (177), 727 (158), and 278 (133) stars, respectively. 


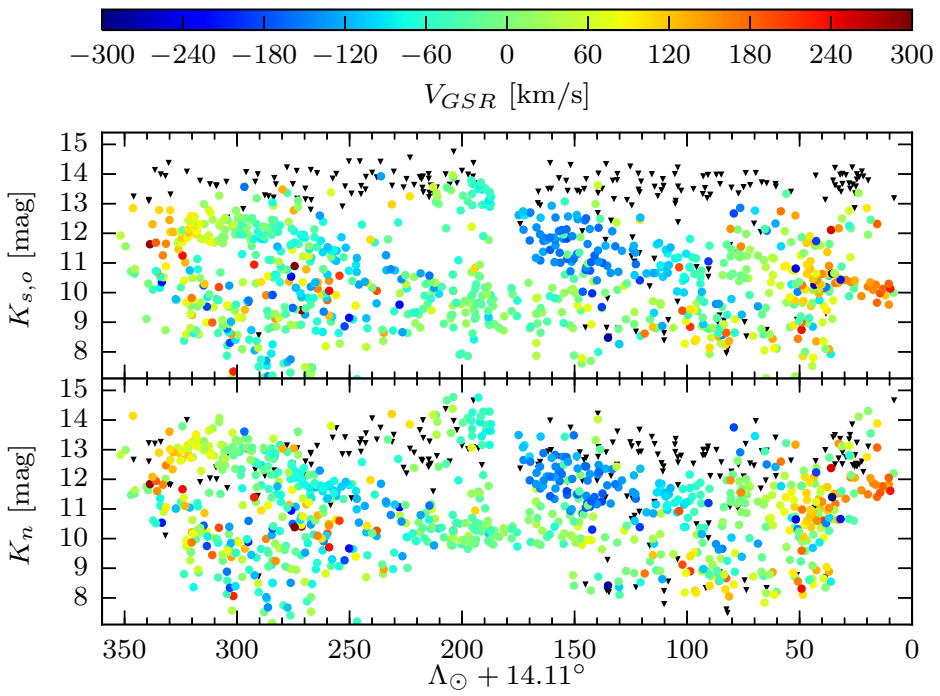

Fig. 3.30.- Comparison between the $K_{s, o}$ and "normalized" $K_{n}$ magnitudes for the sample of giants - color-coded by $V_{G S R}$ - and dwarfs (black triangles). Note how the magnitude spread in $K_{s, o}$ of some structures is reduced in the $K_{n}$ plane. 


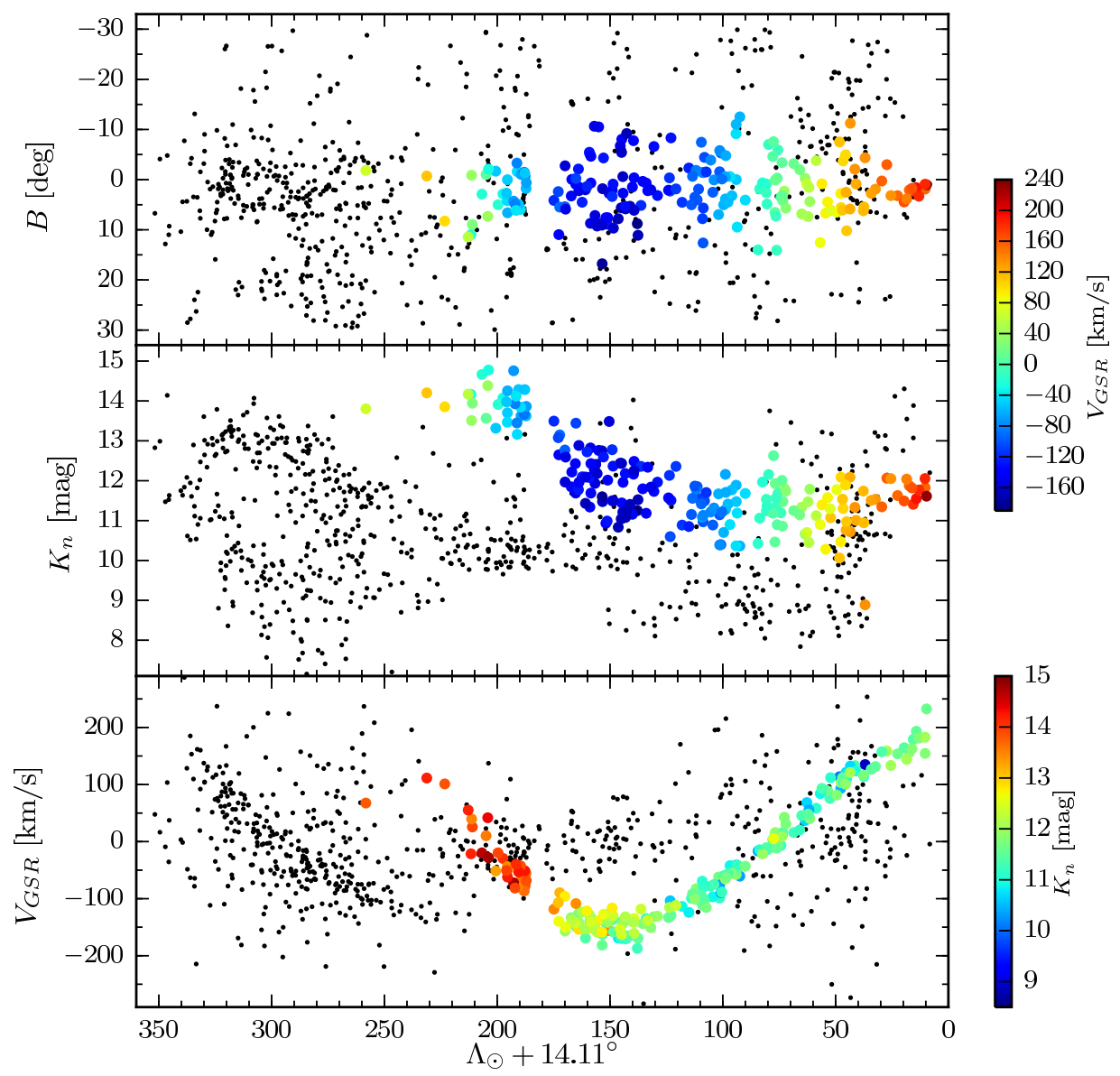

Fig. 3.31. - Stars selected as likely members of Sgr trailing arm. 


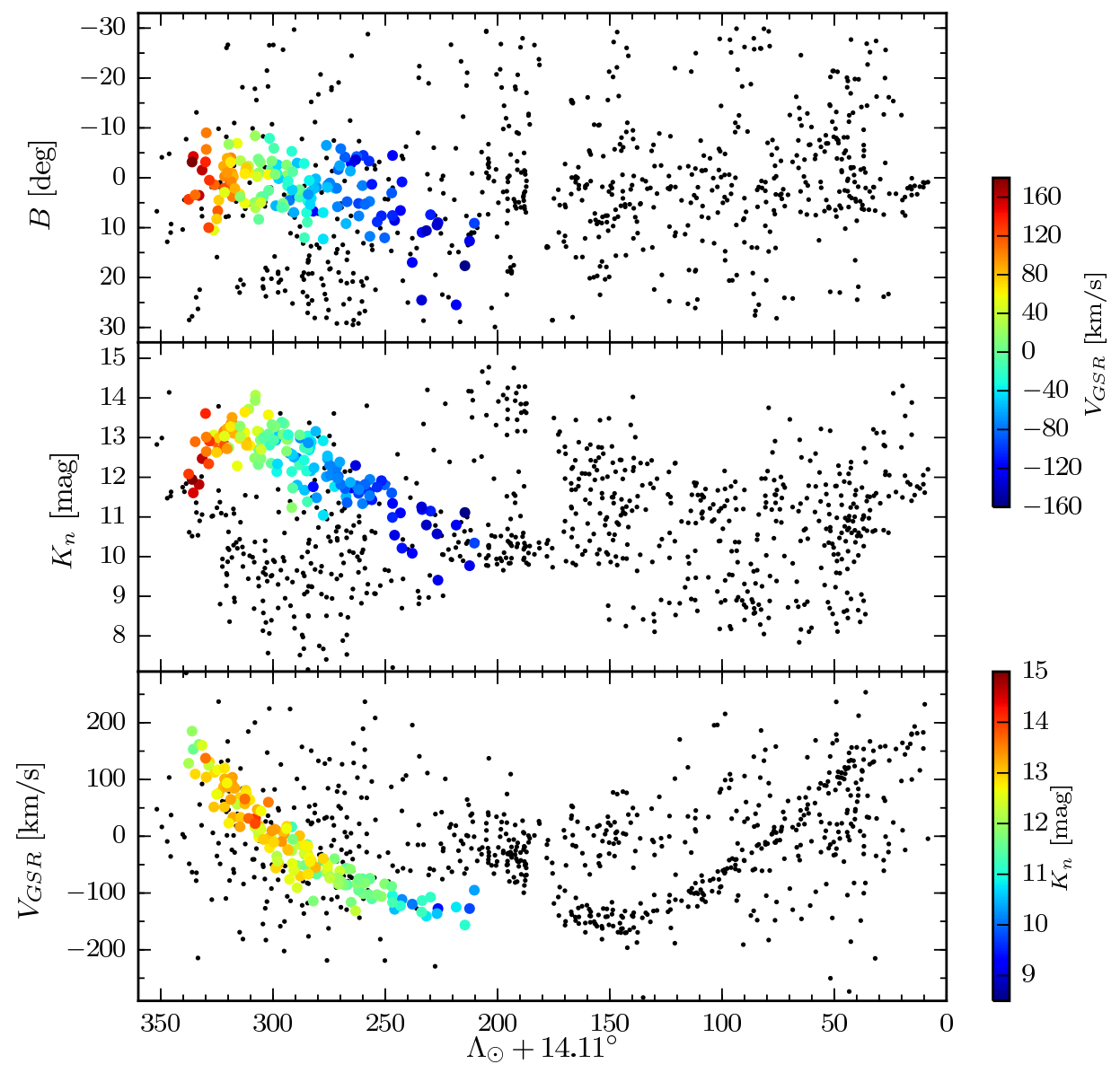

Fig. 3.32. - Stars selected as likely members of Sgr leading arm. 

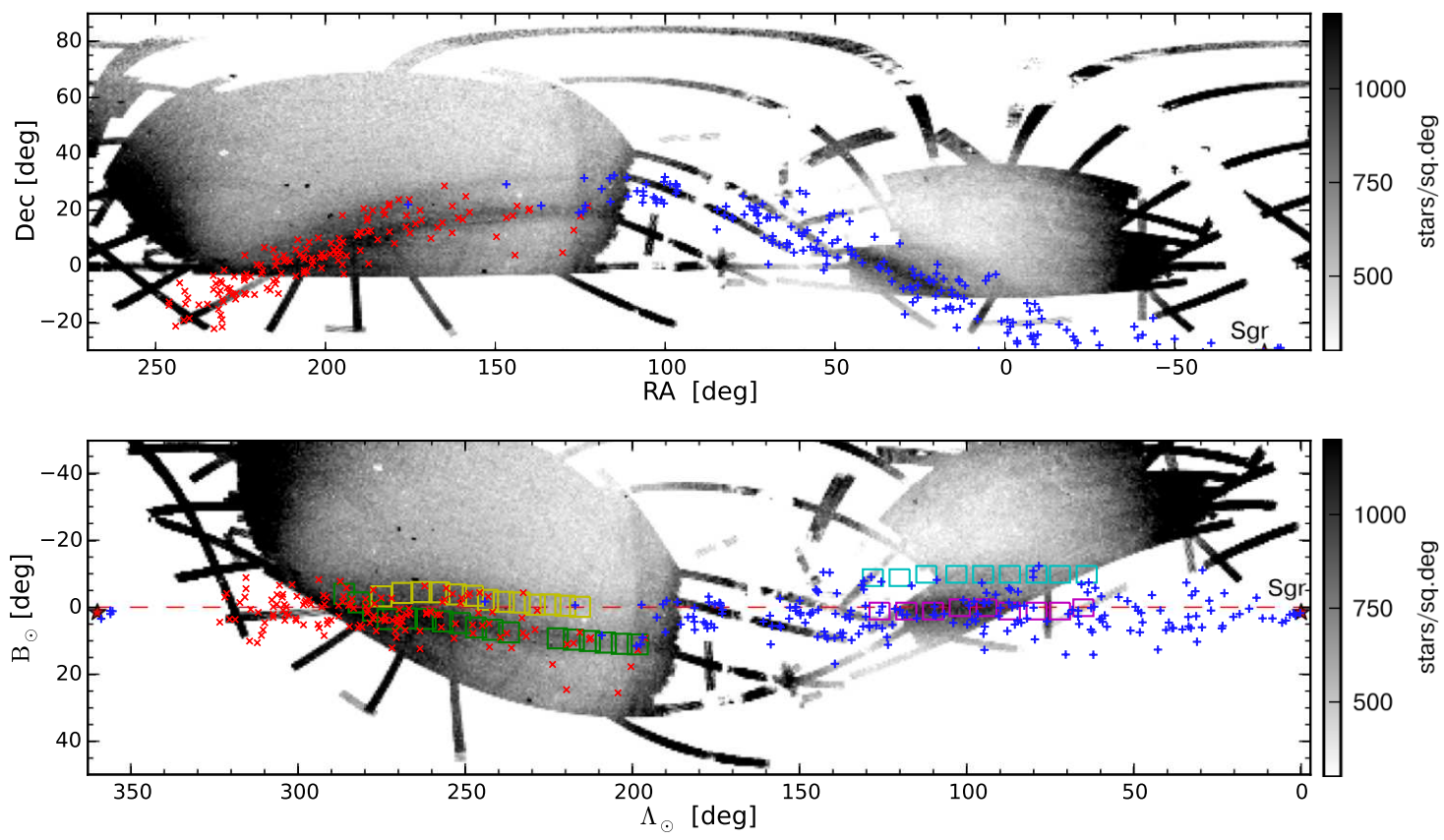

Fig. 3.33. - Distribution on sky of stars selected as likely members of the leading (red crosses) and trailing (blue plus signs), plotted over the northern $(120<R A<250)$ and southern $(45<R A<-10)$ sections of Sgr in the SDSS DR8 footprint, which reveals the bright and faint branches of Sgr. The top panel is in equatorial coordinates, while the bottom panel display the information in the Sgr coordinate system. The later includes the position of the fields used by Belokurov et al. (2006) to trace the bright/faint leading arm in SDSS data (green/yellow squares), and the fields used by Slater et al. (2013) to trace the bright/faint trailing arm in Pan-STARRS1 data. Note how some stars in our sample — selected as likely leading arm members - deviate towards the south of the apparent position of the bright branch of the leading arm at $\left(200<\Lambda_{\odot}<230\right)$. Figure adapted from Koposov et al. (2012). 

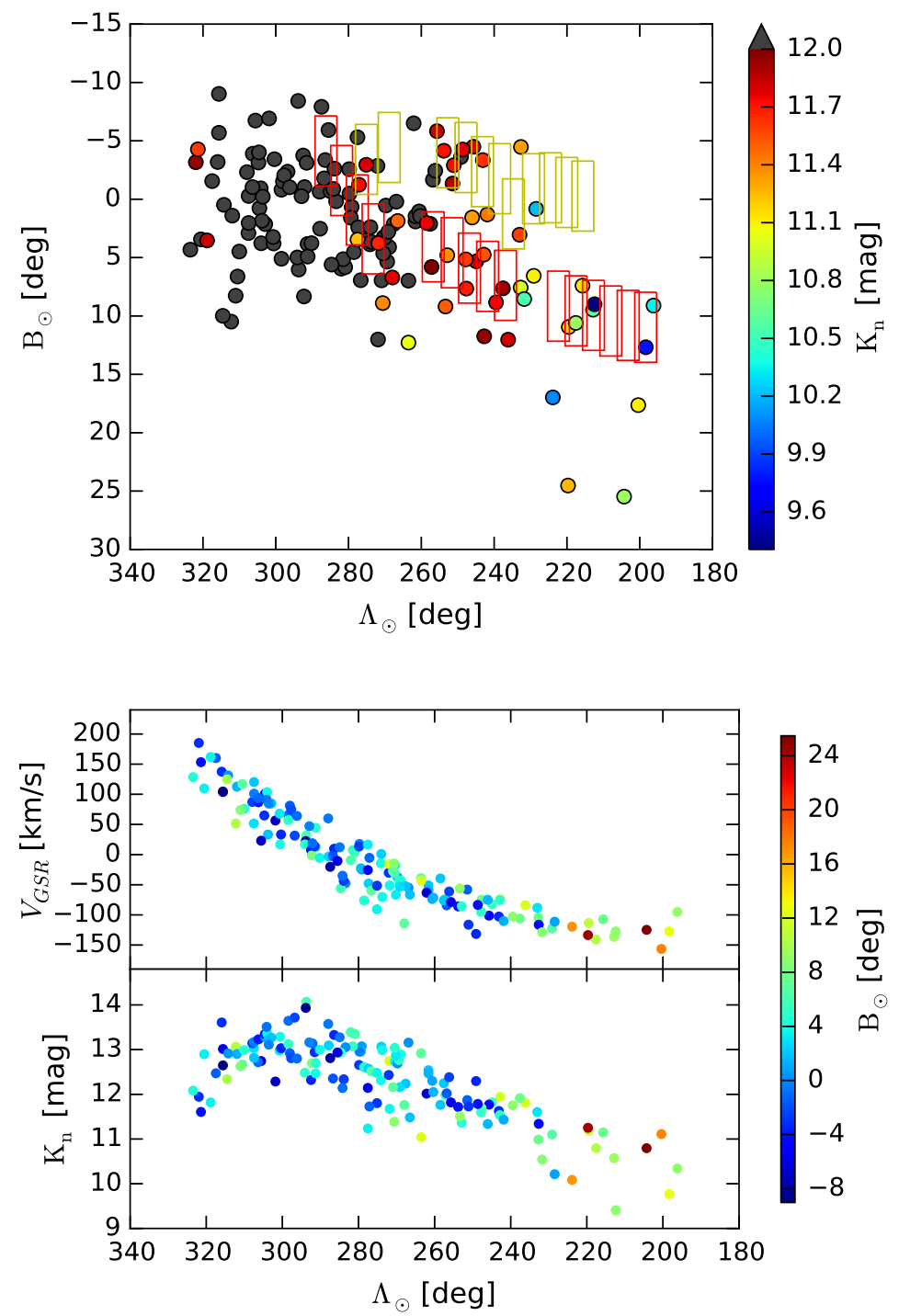

Fig. 3.34. - Top panel: On-sky position —in the Sgr coordinate system - of the leading arm stars color-coded by normalized $K_{n}$ magnitude. The red/yellow squares show the fields of Belokurov et al. (2006) along the bright/faint branches of Sgr. Middle an bottom panels: GSR velocity and normalized $K_{n}$ magnitude along the same range in Sgr longitude as in top panel. The stars are color-coded by $B_{\odot}$ to facilitate cross-matching stars in the top panel. Note that the two stars at highes $B_{\odot}$ have similar $V_{G S R}$ and $K_{n}$ than stars at the latitude of the bright branch. 
Law \& Majewski (2010) model for Sgr leading arm

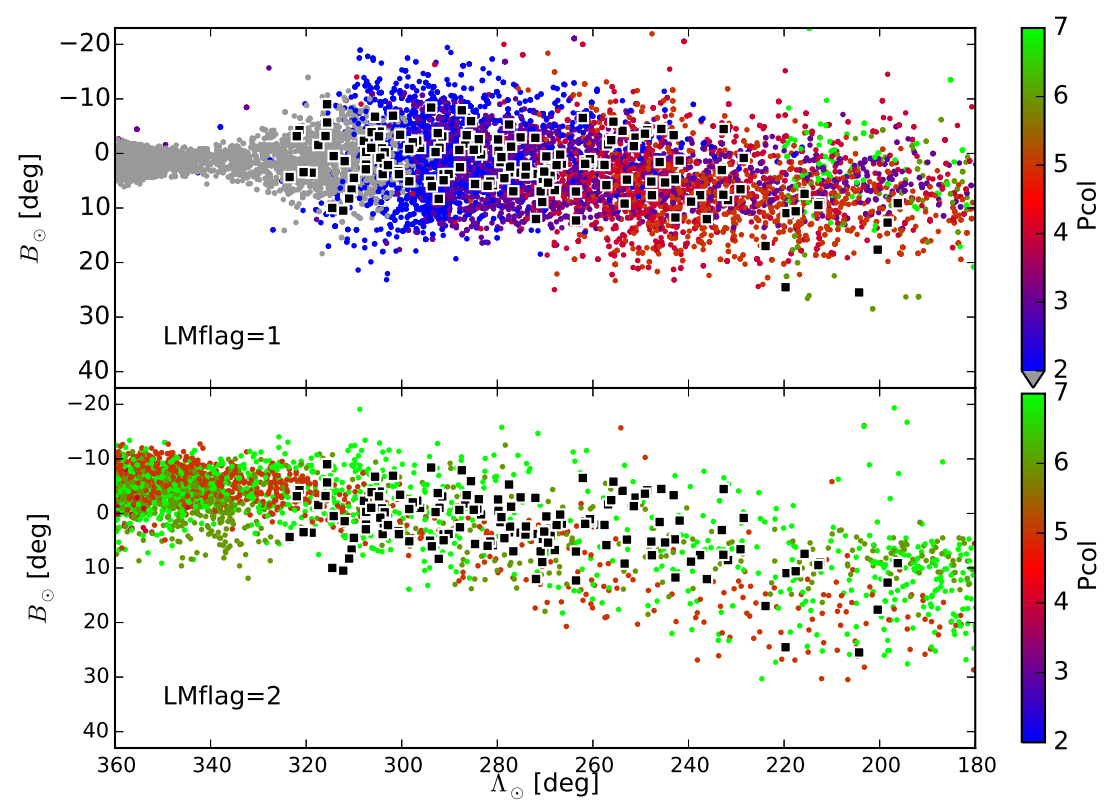

Law \& Majewski (2010) model for Sgr leading arm

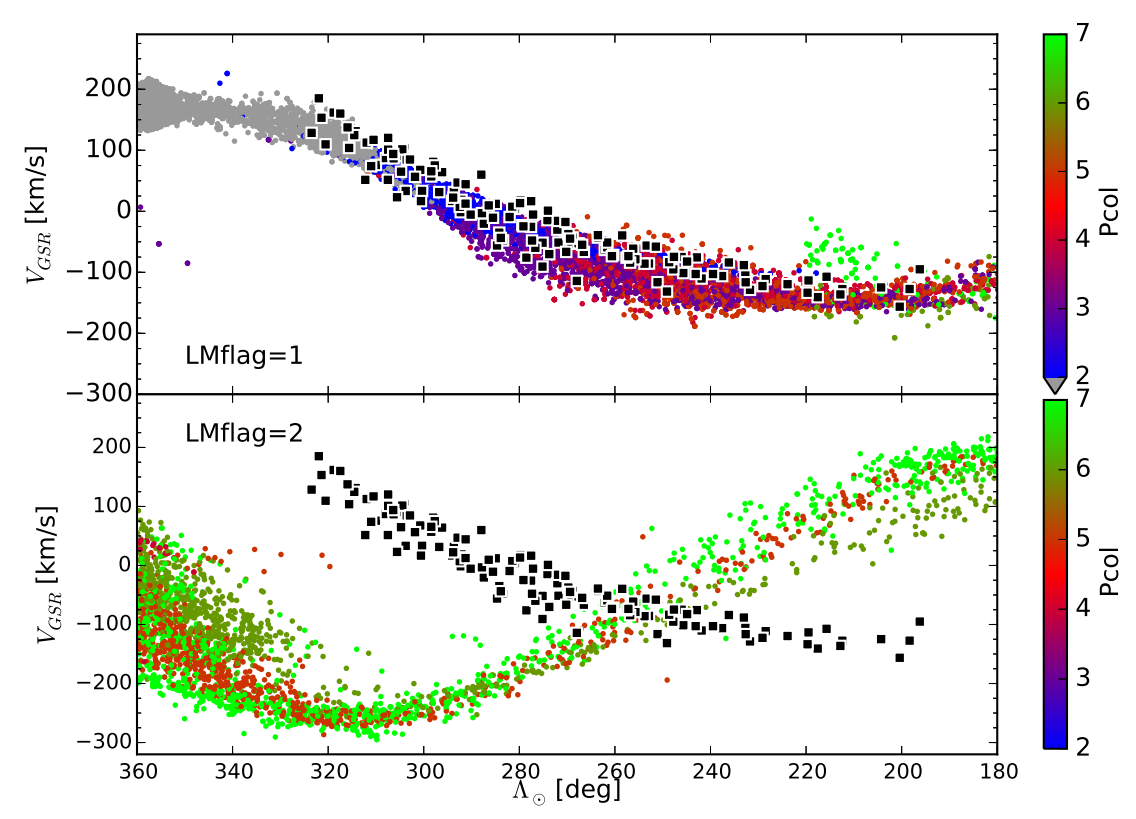

Fig. 3.35. - The Sgr leading arm from the N-body LM10 model. The color code indicates the time of disruption for the debris, as indicated by LM10 in the "Pcol" value. $\mathrm{Pcol}=0$ correspond to debris stripped in the last pericenter passage, $\mathrm{Pcol}=1$ is debris from the previous pericenter passage, and so on. The Lmflag indicates if the debris longitude lie between $0^{\circ}-360^{\circ}(\mathrm{Lmflag}=1)$, or between $360^{\circ}-720^{\circ}(\mathrm{Lmflag}=2)$ from the core. The sample of leading arm M-giants is shown as black squares with white edges. Top two panels: the $\left(\Lambda_{\odot}, B_{\odot}\right)$ position on sky for the first and second wraps of the stream, as indicated by the Lmflag value. Bottom two panels: similar to the top panels, but for the $\left(\Lambda_{\odot}, V_{G S R}\right)$ plane. 


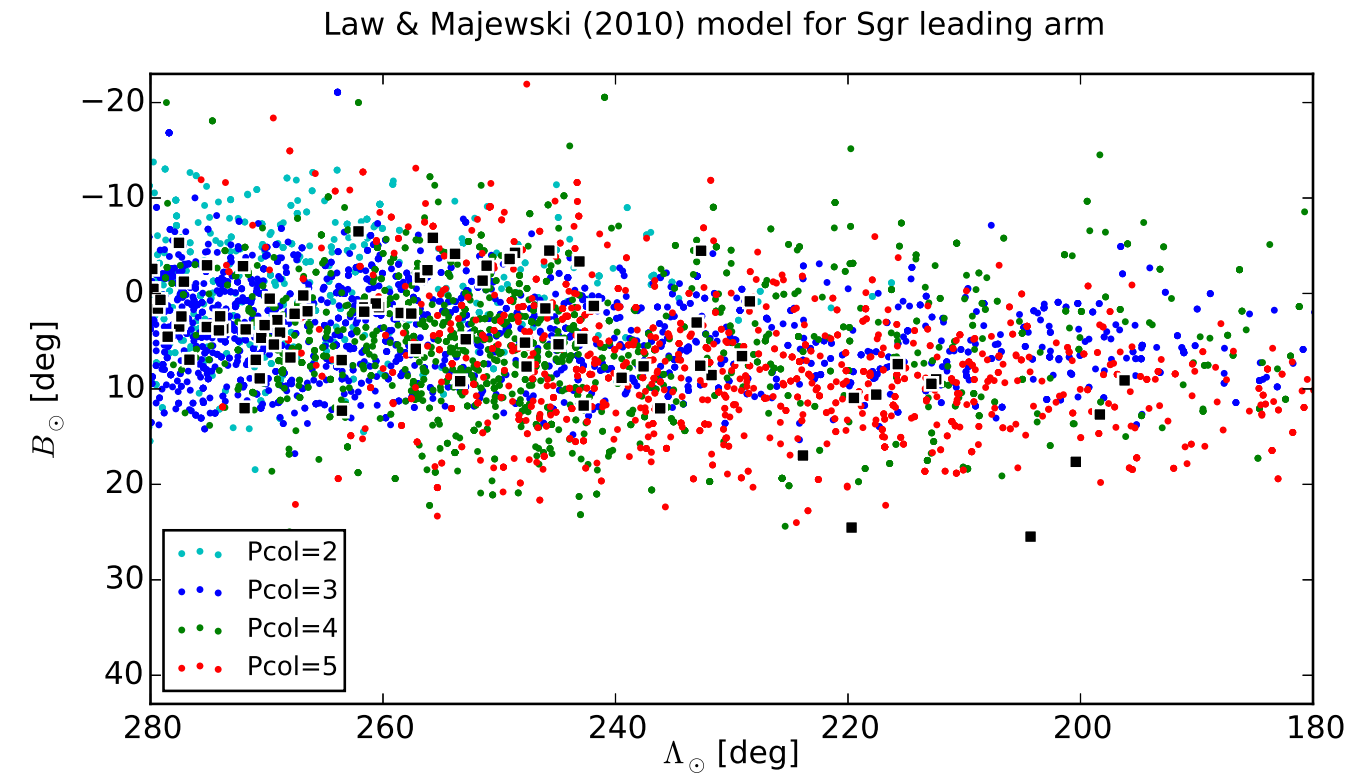

Fig. 3.36. - The leading arm sample and LM10 model particles around the Sgr longitude of the deviation. The more likely origin of the debris in the deviation correspond to $\mathrm{Pcol}=5$. Black squares represent stars in the M-giant sample. 
Law \& Majewski (2010) model for Sgr trailing arm

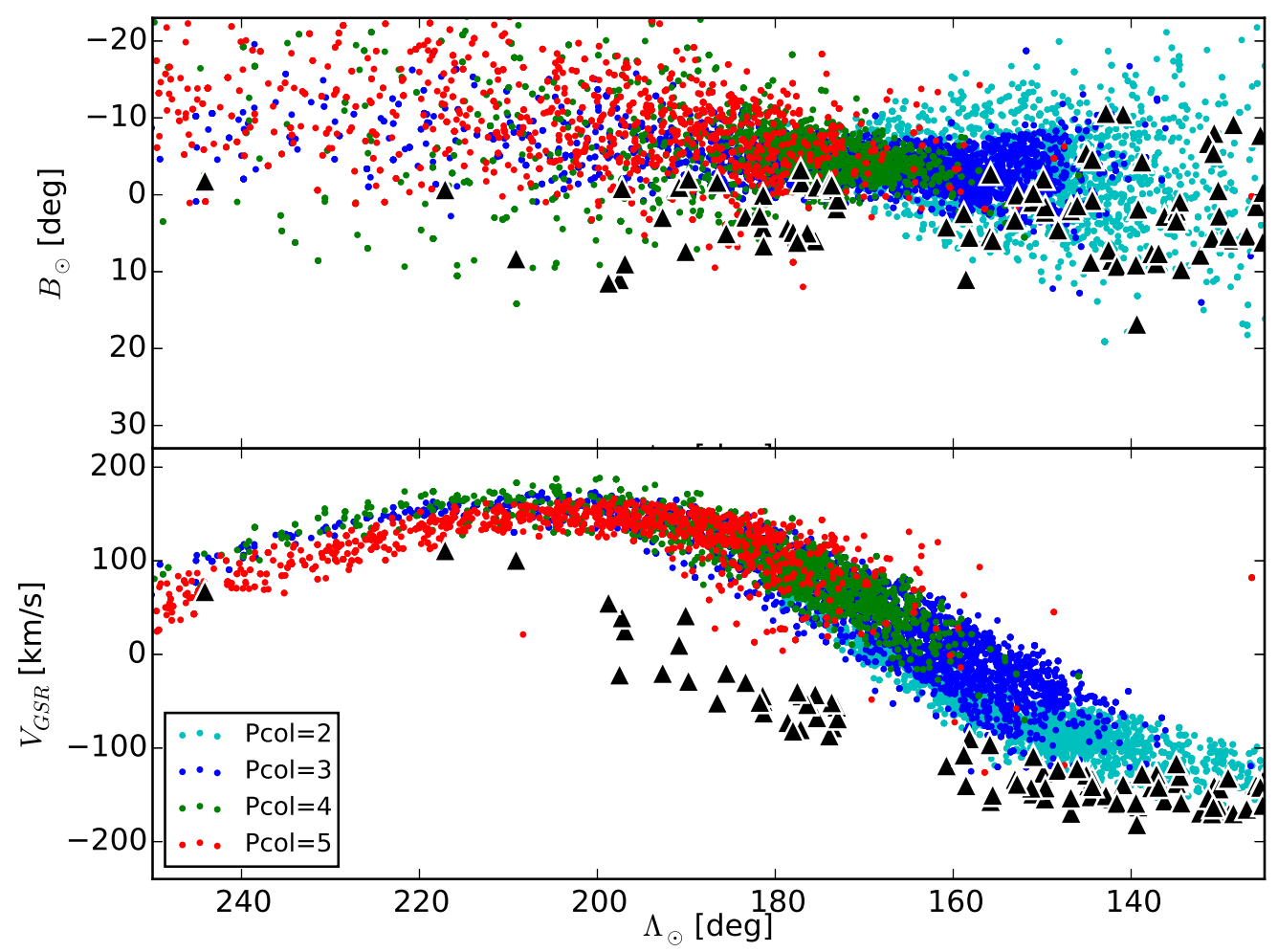

Fig. 3.37. - Similar to Figure 3.36, but for the trailing arm data. In addition to the mismatch in position on sky between the model and data (top panel), we include a panel showing the radial velocity mismatch (bottom panel). The more likely origin of the debris at these longitudes correspond to $\mathrm{Pcol}=4$ and 5 . Black triangles represent stars in the M-giant sample. 


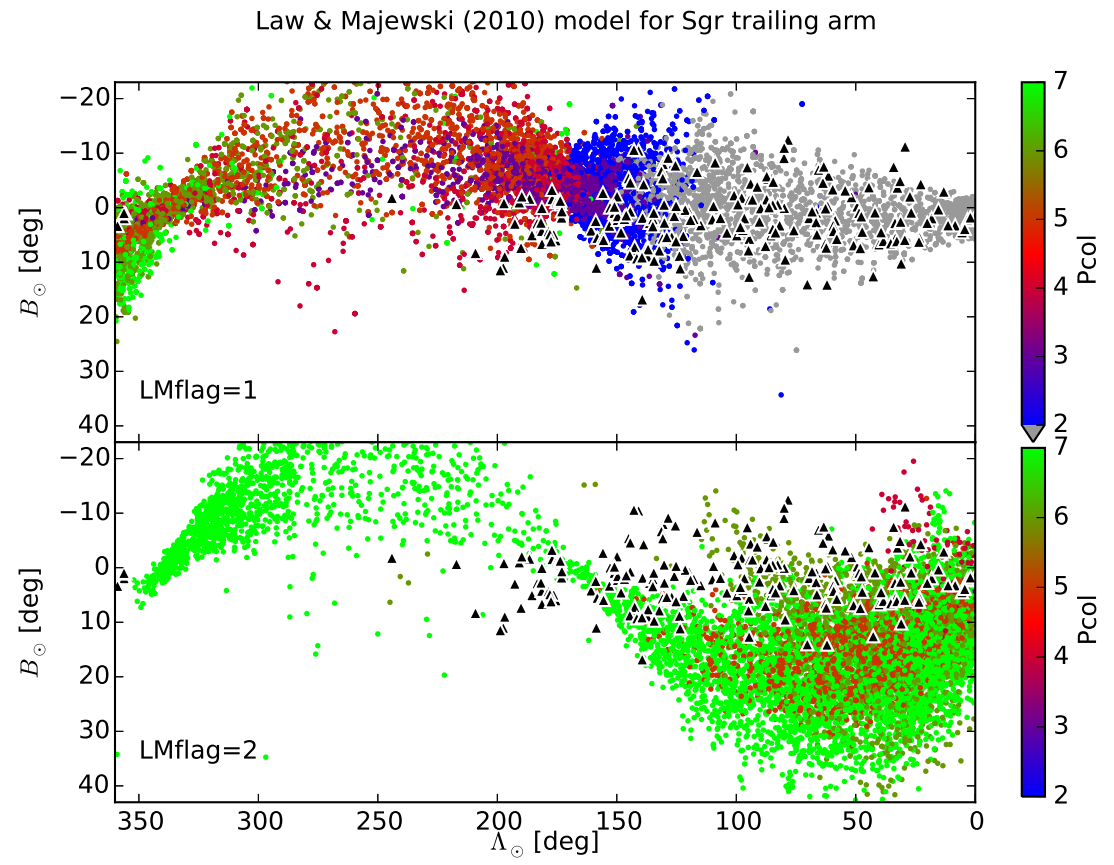

Law \& Majewski (2010) model for Sgr trailing arm

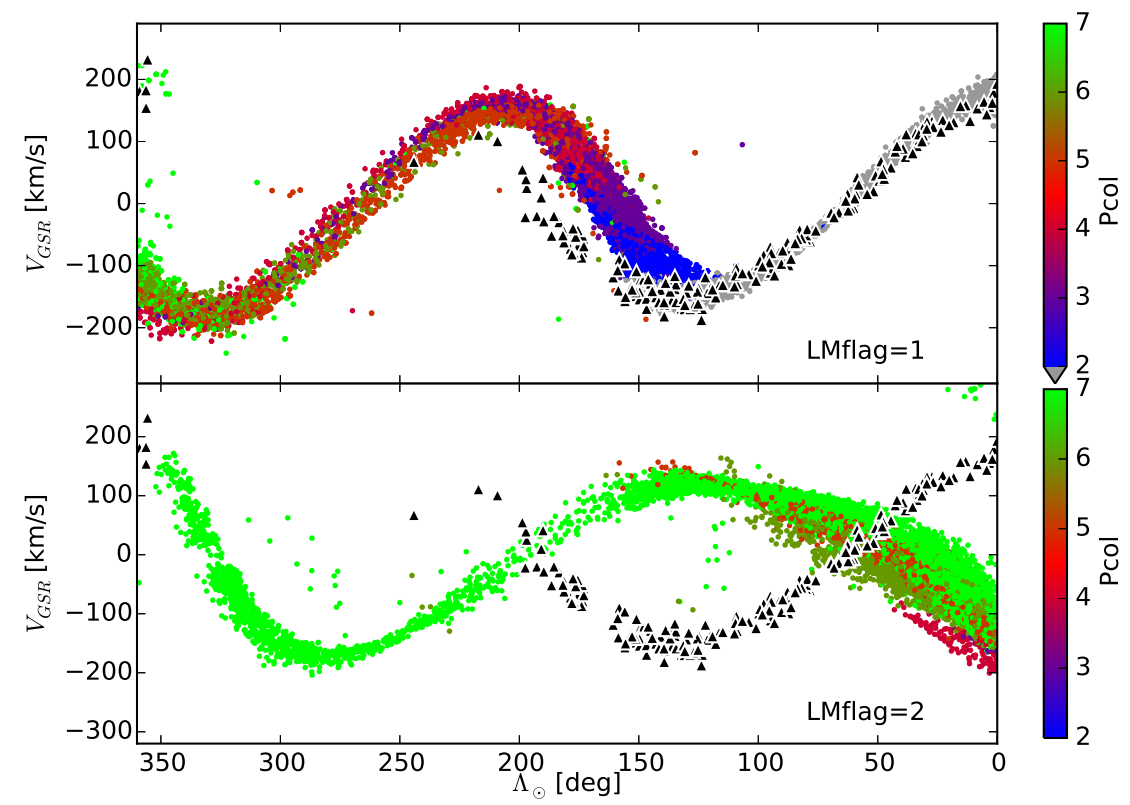

Fig. 3.38. - The Sgr trailing arm from the N-body LM10 model. Black triangles with white edges represent the sample of trailing arm M-giants. See legend of Figure 3.35 for details about the color-coding and panel distribution. 


\subsubsection{GC3 Cell Counts and Orbital Plane Precession}

Johnston et al. (2005, J05 hereafter) showed that the precession of the orbital plane of Sgr can be measured from the on-sky position of the tidal tails. J05 performed a set of N-body simulations that represent the disruption of Sgr accreted by a Milky Waylike galaxy. The later is described by a static 3-component analytic potential — disk, bulge and halo components. The authors ran several N-body simulations varying the halo flatness - described by the parameter $q$ - departing from the spherical shape $(q=1)$ towards oblate $(q<1)$ and prolate configurations $(q>1)$. From these simulations, J05 showed that the angular separation between the orbital poles of the tails is a function of $q$. To measure the orbital pole, J05 used a method of greatcircle cell counts (GC3, Johnston et al. 1996) described below. The J05 simulations showed that the orbital pole derived from GC3 counts for the leading arm is highly and systematically sensitive to changes in $q$, whereas the trailing arm orbital pole is virtually static. In addition, J05 found that the direction of motion of the poles on sky for $q<1$ is the opposite of $q>1$. As a result, the method can be used to constrain whether the halo is oblate or prolate.

We summarize below our implementation of the GC3 cell counts method of Johnston et al. $(1996,2005)$ to measure orbital poles for the M-giant sample. We first divided the sky in a grid of $1^{\circ} \times 1^{\circ}$ cells, where the coordinates of each cell represents the coordinates of a great circle pole in the sky with galactic coordinates $(l, b)$. Next, we calculate the great circle - i.e., the equator - defined by each pole in the grid, and count how many objects lie within a distance $\delta d / 2$ of the great circle -i.e., the cell width is $\delta d$. Finally, we smooth the counts using a Gaussian kernel of $F W H M=5^{\circ}$. This reduces the noise introduced by low number statistics in the cell counts, and eases the determination of the peak of counts, the later of which corresponds to the 
great circle pole.

We determined the peak of the counts by measuring the centroid of the polygon defined by iso-intensity contours, which correspond to iso-counts. To implement this, we use available functions in the scikit-image package (van der Walt et al. 2014) in Python. We first use the find_contours function to calculate a set of iso-intensity contours between $90 \%$ and $99 \%$ of the maximum minus the minimum number of counts in $1 \%$ increments. This function is able to measure contour vertices with fractional-pixel precision through interpolation. We exploit this precision and create a new grid, with an element size equal to $0.1^{\circ} \times 0.1^{\circ}$, to evaluate what elements are inside/outside the contour and set them to 1 or 0 , respectively. This step is performed with the function grid_points_in_poly. Finally, we calculate image moments (through the moments function) to measure the center of mass of the elements set to 1, which corresponds to the center of mass of the iso-intensity contour.

We measured GC3 counts for two data subsets. We create the first subset for measuring the pole separation between the leading and trailing arms, and compare the results against the $q$ versus angular separation simulations presented by J05. We also use this subset to test the how the stars in the deviation affect the pole separation by including or removing these stars from the leading arm data. We begin by constraining the location of stars in this set to $0^{\circ}<\Lambda_{\odot}<170^{\circ}$ for the trailing arm. The leading arm data lies within the range defined by J05 - approximately between $190^{\circ}<\Lambda_{\odot}<330^{\circ}$. To remove stars in the deviation, we set the boundary for the leading arm data to be $B_{\odot}<15^{\circ}$. We ran the GC3 implementation and derive pole counts for both arms. We find that the pole for the leading arm remains unchanged whether or not we include the four stars in the deviation or not, due to the fact that the count peak is weighted by the large number of stars at higher $\Lambda_{\odot}$. 
In order to account for this, we calculated the average $\left(\Lambda_{\odot}, B_{\odot}\right)$ position of leading arm stars in five bins along $\Lambda_{\odot}$ and calculated the pole from GC3 counts. The pole derived through the later approach is sensitive to the inclusion or removal of the stars in the deviation. We show the poles measured for this subset in Figure 3.39.

Next, we calculated the leading-trailing arm pole on-sky distance. We show the measured values along with the results from simulations of J05 in Figure 3.40. There are two important things that can be learned from these figures. First, J05 noted in their simulations that oblate halos produced a leading arm pole with higher $l$ and lower $b$ than the trailing arm pole ${ }^{6}$. We measure a pole separation of $8.2^{\circ}$ and $10.4^{\circ}$ when we exclude and include stars in the deviation, respectively. The relative position and distance that we measured for the poles agree with the oblate halo models of J05. In addition, our results agree relatively well with J05, who measured a separation of $10.4^{\circ}$ for large sample of 2MASS M-giants selected with the photometric method of Majewski et al. (2003). Even though our sample is small, our Sgr giants have been selected using photometric, astrometric and spectroscopic information. Differences in the measured poles may arise from differences in the relative density of stars along the tail, which will weight the position of the peak. Second, we find that including the stars in the deviation increases the pole separation by around 2 degrees. This points towards a slightly more oblate halo (Fig. 3.40).

Next, we make a second subset of data intended for measuring the instantaneous pole position of two sections of the tidal arms. Johnston et al. (2005) also measured the change of instantaneous position of the orbital pole of the core, for both the past and extending 1.5 Gyr from now, for a range of values of $q$. Even though the older debris in our sample may have become disrupted from Sgr more than 3-4 Gyr ago,

\footnotetext{
${ }^{6}$ Note that J05 obtained a $3.3^{\circ}$ pole separation for $q=1$. The smallest pole separation in their simulations was found to be $0.5^{\circ}$ for $q=1.1$, and then the poles switch positions for $q>1.1$.
} 
the orbital phase of the debris in the sample from the core along the orbit is smaller. The photometrically-selected 2MASS sample of Majewski et al. (2003) (analyzed by J05, and included in the sample of this work) reaches an approximate orbital phase of $+0.6(-0.4)$ Gyr for the leading (trailing) arms. Therefore, the signature of the instantaneous pole should be imprinted along the tails. In general, the average phase changes monotonically along $\Lambda_{\odot}$ from the core —although the debris exhibit phase mixing along the orbit.

In the second subset, we also use the leading and trailing arm data to estimate the oblateness/prolateness of the halo from the instantaneous position of the poles at two different orbital phases. We split the leading arm stars into two groups at $\Lambda_{\odot}=140^{\circ}$, and the trailing arm stars at $\Lambda_{\odot}=170^{\circ}$. We chose these limits because the debris on the groups farthest from the core (1) seem to deviate from the LM10 predictions, (2) should have similar orbital phase and (3) have comparable stripping age according to the Pcol values of LM10 (Fig. 3.35, 3.36 and 3.38). Following the same method used in the previous paragraph, we perform the GC3 counts and apply a Gaussian kernel smoothing to estimate the poles of the four data subsets from the counts peak. We present the measured poles in Figure 3.41. In their Figure 4, J05 showed tracks of instantaneous orbital pole positions on the sky (for Sgr core) as a function of orbital phase for different values of $q$. We cannot compare our measured poles directly against the J05 instantaneous poles because we do not know the phase mixing of the debris that comprise our sample. Nevertheless, we can evaluate our results qualitatively. We find that the poles of the leading and trailing arms shift positions following the expected direction for oblate halos, and are incompatible with prolate halos. It is important to note that this conclusion can be made for the pole of both arms independently. 


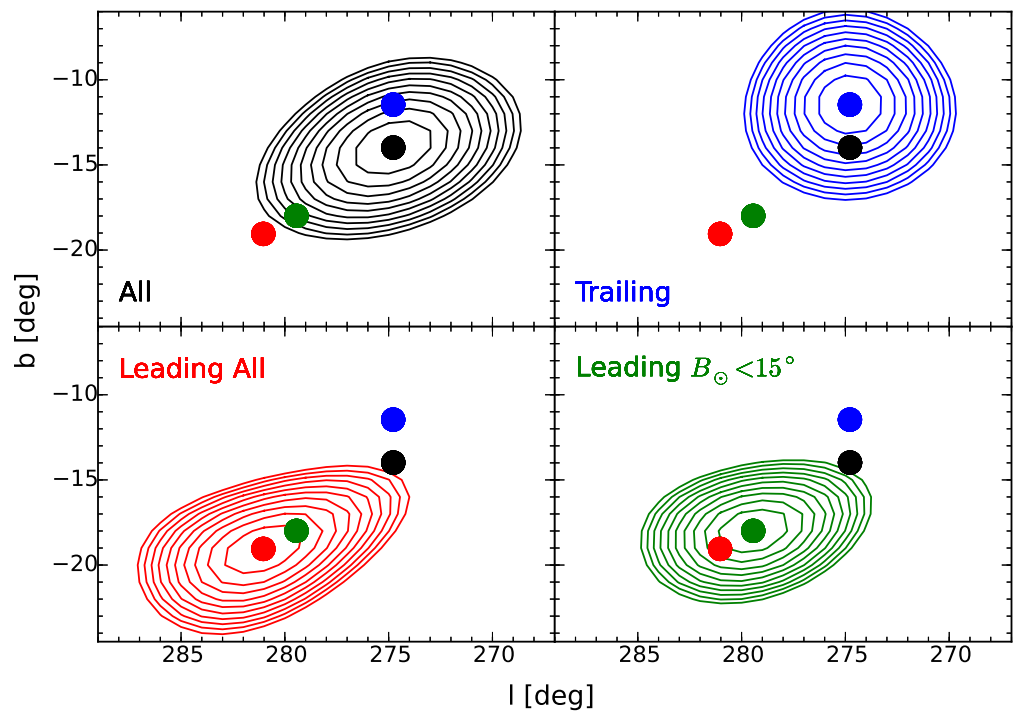

Fig. 3.39. - GC3 poles measured for both, leading and trailing arm debris, calculated for stars within the longitude range considered by Johnston et al. (2005). The contours represent the $90 \%-99 \%$ count levels, while dots represent the pole of the sample, calculated as the centroid of the $99 \%$ level. The colors and text labels indicate the subset of stars used to calculate the respective pole.

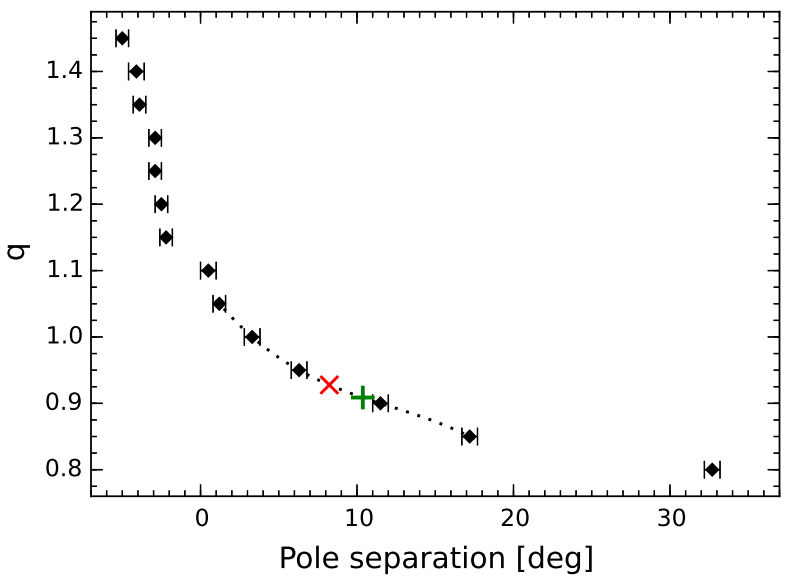

Fig. 3.40.- Pole separation in the context of the pole separation versus $q$ results of Johnston et al. (2005) simulations. The black diamonds indicate the results from simulations, and the dotted line is a polynomial fit to the measurements within $[0.85 \leq$ $q \leq 1.05]$. The red cross and green plus sign show the separation and $q$ value - the later estimated from the polynomial fit - between the leading and trailing arm for the sample that include/exclude the stars in the deviation. 


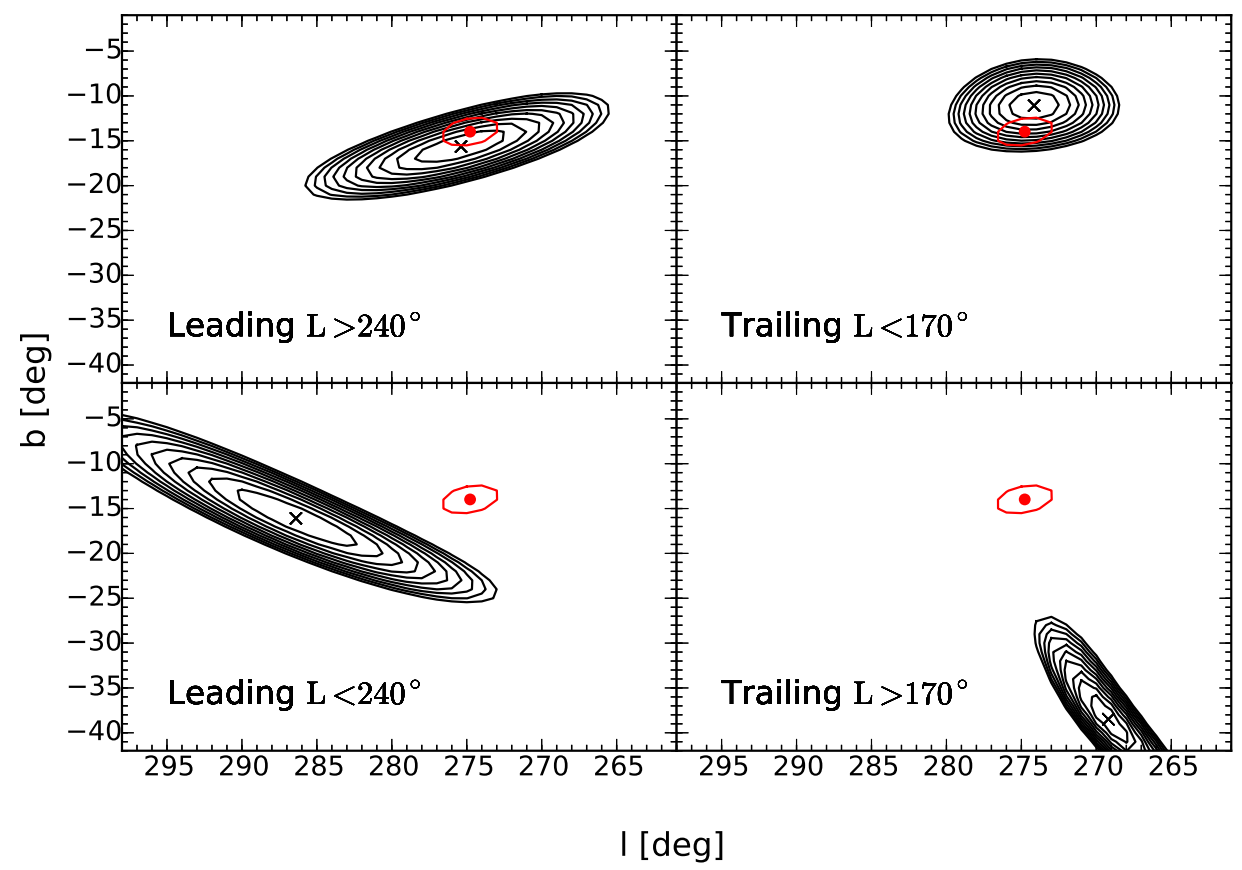

Fig. 3.41.- Instantaneous orbital pole measurements for the leading and trailing arm debris. The upper panels show the debris with orbital phase more similar to Sgr core, while the bottom panels show debris farther from the core and stripped earlier during the MW-Sgr interaction. The contours represent the $90-99 \%$ count peak intensity, for $1 \%$ increments, while black crosses represent the centroid of the $99 \%$ contour. The red contour and circle represent the $99 \%$ contour and centroid, respectively, of the whole M-giant sample. Qualitatively, the relative motion of the poles on the sky agree with an oblate halo, even if we consider the arms separately. 
The halo flattening that we have derived in this work is in good agreement with the measurements of Johnston et al. (2005). However, this halo flattening is not consistent with the best model of Sgr disruption to date (LM10). LM10 model (also Law et al. 2009, work) relies on a triaxial halo with a peculiar axis orientation a mildly oblate tilted ellipsoid, with its major and minor axis along the Galactic plane and intermediate axis perpendicular to the Galactic disk - with respect to the cosmological halos found in simulations. In addition, Slater et al. (2013) and Deg \& Widrow (2014) find that the LM10 halo configuration explains the Sgr debris location.

On the other hand, the LM10 halo configuration has raised some important questions. For instance, (Debattista et al. 2013) showed that the stellar disc is unstable under the orientation of the LM10 halo. They also include references that find stellar orbits in disks are stable under triaxial halos if the disk rotation axis is aligned with either the major or minor axis of the halo, but not with the intermediate axis as in LM10. Other solutions to the halo shape problem have been explored. One of them is presented by Vera-Ciro \& Helmi (2013), who propose an oblate halo potential $\left(q_{z}=0.9\right)$ dominating the central region of the Galaxy $-r<10 \mathrm{kpc}-$ and then slowly transitioning into the LM10 potential at larger radii. This model ensures that the disk is within a potential that guarantees a stable configuration. However, their most interesting finding regards the effect that Large Magellanic Cloud (LMC) could have in Sgr disruption. The authors argue that the effects of the LMC should not be neglected on Sgr simulations, and show that (1) the mass of the LM10 halo interior to the current Sgr position is comparable to the LMC mass $\left(M_{\text {halo }} \sim M_{L M C} \sim 10^{11}\right)$, and that (2) the torque exerted by the LMC points in the same direction of the torque induced by the LM10 halo. Gómez et al. (2015) also tests the influence of the LMC on Sgr disruption. In addition to the passage of the LMC, they allow the barycenter 
of the MW to freely react to the LMC passage. They find that the MW barycenter could have shifted as much as $15 \mathrm{kpc}$ in the last 0.3-05 Gyr. The authors do not attempt to solve for the initial conditions that would produce the current Sgr system configuration, but rather explore the effects of the LMC and the non-inertial MW frame in simulations.

Interestingly, Gómez et al. (2015) find that the orbital pole of Sgr changes by $9^{\circ}$ when the MW can react freely to the perturbation of the LMC. In addition, they arrive to this result without using prolate/oblate halo configurations but a NavarroFrenk-White (Navarro et al. 1996) DM profile. Therefore, it is possible that the LM10 potential, which is static and fixed in the Galactocentric frame, has been successful in modeling the stream as its parameters have been adjusted to artificially represent the effects of the LMC passage. This raises the question whether the measured orbital pole precession is the result of a more oblate halo, or is the result of the LMC+MW interaction. The answer to this question probably requires detailed modeling of the problem which is beyond the scope of this work.

\subsubsection{Metallicity Gradient Along the Tidal Arms}

In addition, we present metallicity measurements along the stream, obtained with the CaT method calibrated through isochrones, measured as described in Section 3.2.5. The results are shown in Figures 3.42 and 3.43, for the stars selected as trailing and leading arms, respectively. We only include stars whose SNR, measured on the continua bandpasses of the three CaT lines, is greater than 25. Because the spectroscopic campaign was originally intended for measuring radial velocities only, a large fraction of our sample have $\mathrm{SNR}<25$. As a consequence, only 54 and 50 stars pass the cut for the trailing and leading arms, respectively. We report the metallicities 
derived from the CaT $W$ measurements where we have fitted for the continuum level within the continuum bandpasses - we also derived metallicities for $W$ as measured with the continuum fixed to 1.0. We prefer the fitted continuum approach because we used different low- and high-sigma rejection for the background estimation during the spectra normalization with the hope that we could reduce the effect of spectral lines in the continuum placement. However, the metallicities derived with the continuum level set at 1.0 are significantly greater than the metallicities derived from the fitted continuum. For instance, the mean difference $\left\langle[\mathrm{Fe} / \mathrm{H}]_{\text {fixed }}-[\mathrm{Fe} / \mathrm{H}]_{\text {fitted }}\right\rangle$ between metallicity from fixed and fitted continuum is $0.57 \pm 0.44$ and $0.48 \pm 0.27$ for the trailing and leading arms, where the uncertainty represents the standard deviation of the sample. We do not see any dependence of the metallicity difference with respect to SNR, metallicity and metallicity uncertainty. Therefore, the difference seems to be a systematic offset introduced by consistently setting the continuum higher than the true value when we set the lower and higher rejection sigmas for the normalization. Thus, we use the metallicity values derived from the fitted continuum as the method follows the more common procedure to calculate the continuum level using bandpasses.

We fit the metallicity trends along both arms to measure the metallicity gradients. For the trailing arm we obtain

$$
[\mathrm{Fe} / \mathrm{H}]_{\text {trail }}=-0.0315( \pm 0.0202) \frac{\Lambda_{\odot}}{\left[10^{\circ}\right]}-0.2752( \pm 0.3245),
$$

for $\Lambda_{\odot} \in\left[31^{\circ}, 244^{\circ}\right]$. On the other hand, for the leading arm we obtain

$$
[F e / H]_{l e a d}=0.0264( \pm 0.0192) \frac{\Lambda_{\odot}}{\left[10^{\circ}\right]}-1.1795( \pm 0.5412),
$$

for $\Lambda_{\odot} \in\left[212.4^{\circ}, 323.4^{\circ}\right]$. In both cases, the metallicity decreases proportionally as 
angular distance increases along the arm from the core (note that the leading arm relation implies Sgr core to be at $\Lambda_{\odot}=360^{\circ}$ ). In addition, the spread in metallicity is large. Therefore, we proceed with caution before we derive conclusions. In one hand, the gradient and spread in metallicity have been observed in some selected fields along the tails (and radially along the core). For instance, Chou et al. (2007) measured metallicities for a sample of leading arm M-giants via high-resolution spectroscopy. They found that the metallicity decreases from a mean metallicity of -0.4 dex at the core to around -0.7 dex at $60^{\circ}-120^{\circ}$ from the core. Furthermore, Carlin et al. (2012) also finds a lower metallicity compared to Sgr core, using low-resolution spectra in four fields along the stream. However, found metallicity to be constant along $60^{\circ}$ of the stream, but with an spread of 0.5 dex, which theyobtain from mostly MSTO stars. This type of stars are not biased towards higher metallicities as M-giants do.

If we extrapolate the gradients towards the core - i.e., $\Lambda_{\odot}=0^{\circ}$ and $\Lambda_{\odot}=360^{\circ}$ for the trailing and leading arms, respectively - we find $[\mathrm{Fe} / \mathrm{H}]_{\text {trail,core }}=-0.28$ and $[\mathrm{Fe} / \mathrm{H}]_{\text {lead,core }}=-0.23$ dex. Interestingly, these extrapolated metallicities -if we conjecture that the linear gradient is real and extends up to the core - are in relatively well agreement with the -0.4 dex median metallicity of the core measured from high-resolution spectra by (Chou et al. 2007), who also found that there is a dominant core population that peaks at -0.3 dex. In addition, the extrapolated CaT metallicity values from the leading and trailing arm gradients are consistent with each other within their errors.

In general, our metallicity measurements obtained through the CaT technique developed in Chapter 2 seem to agree with the trends observed by other authors. However, our spectra have lower dispersion $\left(\sim 1-1.5 \AA \mathrm{pix}^{-1}\right)$ compared to the dispersion used by Warren \& Cole $(2009)^{7}$ in their CaT calibration, which is where we

\footnotetext{
${ }^{7}$ note that they provide the spectral dispersion as spectral resolution
} 
obtained the calibration data for Chapter 2. In addition, we estimated the SNR of the spectra as the square root of the mean counts in the continuum bandpasses. This may be too optimistic (for example, it does not consider the read-noise of the detector), which results in overestimated metallicity uncertainties.

\subsection{Conclusions}

We have identified a clean sample of M-giants belonging to the leading and trailing tails of the Sgr system. We found a group of four stars at the end of the known extension of the leading arm that deviate from the trend followed by the A branch of the arm. We conjecture that these stars are part of Sgr, because the four stars agree with the stars in the A branch in radial velocity and normalize $K_{n}$ magnitude - a gauge of distance. Even if these stars are not Sgr, they may belong to an unknown halo substructure. The later would also be an interesting outcome. In addition, we use CG3 counts to derive orbital poles for the debris, and find that the results favor an oblate over a prolate Galactic halo. The extension of the trailing arm debris towards the north also favor this type of halo. Including the stars in the deviation in the GC3 analysis suggest a slightly more oblate halo. Finally, we find consistent metallicity gradients along the leading and trailing arms. The gradients have large dispersion, which can be the result of the intrinsic spread combined with large uncertainties induced by the resolution and SNR of our data. 


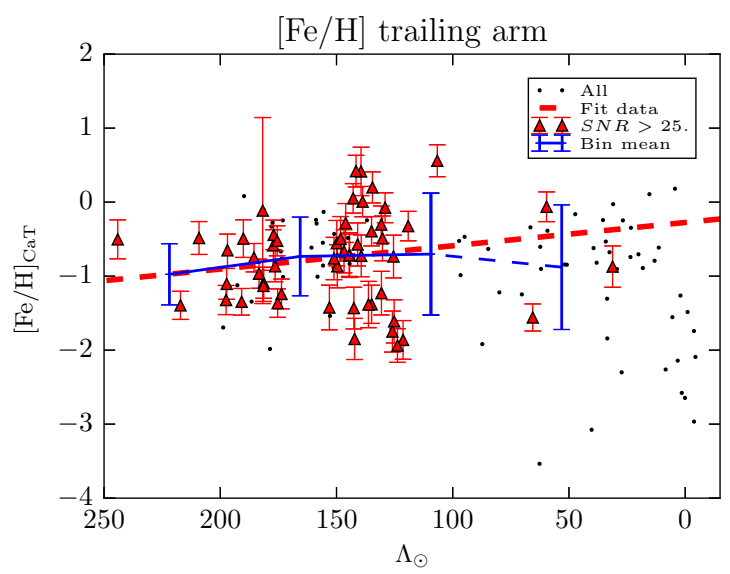

Fig. 3.42.- Metallicity values for the stars selected as trailing arm members. The black dots show all the stars selected as arm likely members that have CaT measurements. Red triangles show the subset of stars with $S N R>25$ for the three Ca lines, with error bars indicating metallicity uncertainty. The red segmented line is a weighted-fit for the stars with $S N R>25$, while the blue marks and error bars indicate the weighted mean and weighted standard deviation for 4 bins (55-degree wide bins). Note that the bin centered at $\lambda_{\odot} \sim 50^{\circ}$ has only three stars, which may cause the deviation from the global trend.

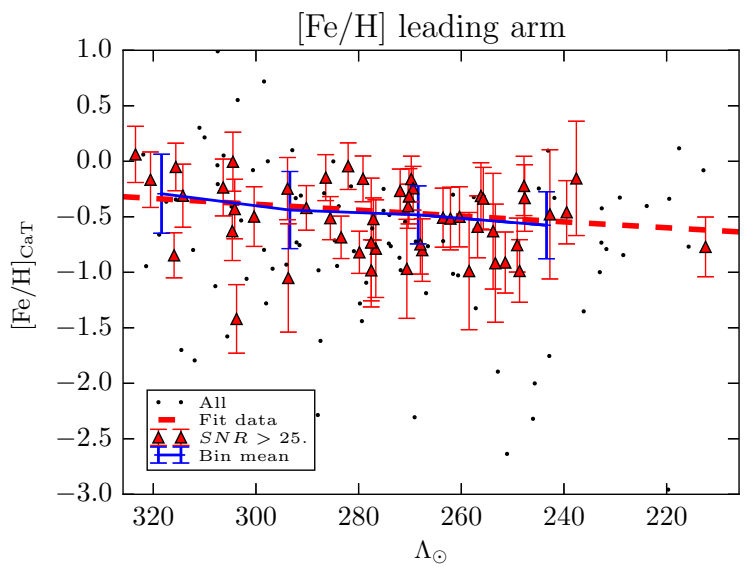

Fig. 3.43. - Same as Figure 3.42, but for stars selected as trailing arm members. The bin width for the blue marks is 25 degrees. 


\section{Chapter 4}

\section{Solar Motion and the Local}

\section{Standard of Rest as seen in the}

\section{Grid Giant Star Survey}

\subsection{Introduction}

The study of the Solar motion and the MW rotation has historically been an important topic of Galactic astronomy. This quantity set important constraints in the Dark Matter (DM hereafter) content in the Solar neighborhood, which is key in the design of experiments of direct-detection of DM. Read (2014) provides a recent review on this topic. Therefore, special attention has been given to understanding the Galactic rotation, the Solar motion and its distance to the Galactic center.

It is common convention to define the Solar motion as the 3-D velocity components $(U, V, W)_{\odot}$, in a sun-centric - but rotating - Cartesian frame, referred to the Local Standard of Rest (LSR). The LSR represents "the current velocity of a fictional particle that moves around the plane of the Milky Way on the closed orbit in the 
plane that passes through the present location of the Sun. If the Milky Way is axisymmetric, this orbit is circular, otherwise it has an oval shape" (Binney \& Merrifield 1998). If more than one orbit under this condition is possible, then the most circular one is chosen. Other stars in the solar neighborhood have their own peculiar motion $(U, V, W)_{*}$.

The Galactic rotation curve is often estimated using the Doppler motions of HI, $\mathrm{CO}, \mathrm{H}_{2}$ or other gas tracers(e.g., Merrifield 1992). In addition, the rotation curve is usually measured with respect to a particular tracer of a specific stellar population. Stellar velocities are measured either from proper motions, radial velocities, or both (usually referred to as "space velocities").

Even though proper motions and parallaxes have been used for a long time to measure the Solar motion and Galactic rotation (e.g., Strömberg 1946), parallaxes are only reliable for nearby stars. Proper motions — when combined with parallaxes to derive accurate transverse velocities - are usually used to measure the kinematics and Solar motion within the Solar neighborhood, , because uncertainties in transverse velocities scale with distance. For instance, the work of Strömberg (1946) uses a sample that includes stars within 20 pc. Even with the precision of the Hipparcos satellite, the sample used by Dehnen \& Binney (1998) has a mean distance of only $72 \mathrm{pc}$.

Stellar motions also can be derived via radial velocities (RV, hereafter) only. Stellar RVs present the advantage that their uncertainty is independent of distance, but depends on the spectrograph resolution. Therefore, RVs of stars at large distance — several kpc from the Sun - can be measured accurately. However, the main disadvantages are the uncertainty in distance to the stars - which usually is derived through photometric or spectroscopic parallaxes-, and that spectroscopy has histor- 
ically been an "expensive" technique in terms of telescope time. Nevertheless, the last decade has seen the development and implementation of large-scale surveys of radial velocity of stars in the MW, with multiplexed observations through fiber-fed spectrographs. Among these surveys, are the Radial Velocity Experiment (RAVE) (Steinmetz et al. 2006; Zwitter et al. 2008), the Sloan Extension for Galactic Understanding and Exploration (SEGUE) (Yanny et al. 2009) and SEGUE-2, the Apache Point Observatory Galactic Evolution Experiment (APOGEE) (Majewski et al. 2015) and APOGEE-2, and the Large sky Area Multi-Object fiber Spectroscopic Telescope (LAMOST) survey (Cui et al. 2012; Zhao et al. 2012). After extensive observing campaigns, these surveys have or will have measured radial velocities for several hundreds of thousands and even millions of stars. These data have been used to measure the Solar motion and Galactic rotation, but the results do not often agree.

In any case, these large surveys of radial velocities have the advantage of sampling vast regions of the Galaxy, and not only the Solar neighborhood. For instance, measurements from nearby samples may be affected by local irregularities, such as moving groups. These local measurements therefore require discrimination to discern between moving groups and the stars that follow the LSR (e.g., Francis \& Anderson 2014).

Therefore, precise values for these velocities are under debate, even with the wealth of data available nowadays. In the following sections of this chapter, we present preliminary results of ongoing work where we utilize the Giant Grid Star Survey to measure the peculiar motion of the Sun and estimate Galactic rotation. The work has been conducted under collaboration with Dr. Ralph Schönrich (University of Oxford, U.K.). 


\subsection{The Grid Giant Star Survey}

\subsubsection{Photometric Campaign}

The Grid Giant Star Survey (GGSS hereafter) is an all-sky, partially-filled photometric and spectroscopic survey of K-giant stars (Patterson et al. 2001). The survey was initially planned to provide the Astrometric Grid (AG) for the (now canceled) Space Interferometry Mission (SIM) that was expected to launch in 2009. SIM was designed to measure stellar centroids for sources relative to the AG with an astrometric precision of $10 \mu$ as per observation. After the proposed 5 year campaign, the combined precision would have reached $4 \mu$ as. NASA funded the creation of the GGSS as a fundamental component of the AG.

The SIM project had hard requirements that influenced the selection of the sources that would comprise the grid. The photometric limit was set by photometric precision, SNR, and AG density requirements. In addition, the AG must be astrometrically stable. As Patterson et al. (2001) note, sources of potential astrometric instability include stellar, brown dwarf and planetary companions, and photospheric activity that cause flares and starspots that result in "photocenter wonder". The last point became important because SIM could have resolved the photospheres of $V<13$ giant stars.

The GGSS, led by UVa (Majewski+Patterson), was designed to address the AG problem. The survey targeted $\mathrm{K}$ giant stars of sub-solar metallicities and with $V<13$. Such stars are inherently bright; thus, K-giants are farther than other giants at the same magnitude, reducing any potential astrometric wobbling of the star. In addition, sub-solar K-giants are less likely to host planetary companions (Fischer \& Valenti 2005). Finally, at $V<13$, the density of such stars is high enough to construct a 
grid of 1302 "bricks" containing sufficient sources within the satellite's $15^{\circ}$ "field of regard". In 1998, NASA approved a proposal to execute the GGSS campaign in the Southern Hemisphere. Additionally, a second proposal was approved in 1999 to carry out the Northern Hemisphere campaign.

Because the GGSS targets late-type giant stars, luminosity class discrimination becomes critical. The difference in luminosity between giants and dwarfs could reach about 10 magnitudes. Therefore, the GGSS used a photometric technique that discriminates between K-type giants and dwarfs. Moreover, within the groups of photometrically selected giants, it is also possible to identify those that are likely to be of lower metallicity using the same photometry.

The photometric technique takes advantage of the gravity-sensitivity of the $\mathrm{MgH}$ and Mg I "b" line around 5,200 $\AA$ (Fig. 4.1). The method is based on that of Geisler (1984), who discriminates G- and K-giants form dwarfs with Washington $\left(T_{1}-T_{2}\right)_{o}$ versus $(M-D D O 51)_{o}$ photometry. In this four-filter system, the $\left(T_{1}-T_{2}\right)_{o}$ color is sensitive to effective temperature, whereas the $(M-D D O 51)_{o}$ is sensitive to gravity. The medium-band DDO51 filter is designed to measure the $\mathrm{MgH}$ feature, while the broad-band $M$ filter works as a the continuum estimator. The GGSS photometric selection is based on Geisler's system, but using three filters. Majewski et al. (2000) showed that the $T_{1}-T_{2}$ color correlates monotonically with $M-T_{2}$ for most stars. As a consequence, the simplified - and therefore more efficient in terms of telescope use$\left(M-T_{2}\right)$ versus $(M-D D O 51)$ plane is just as effective for giant/dwarf discrimination of F-K type stars (Fig. 4.2).

Within the SIM AG requirements, we find that calibration stars should be available several degrees from any direction in the sky. This condition shapes the distribution of GGSS fields, described as a "partially-filled, all-sky survey". As Patterson 


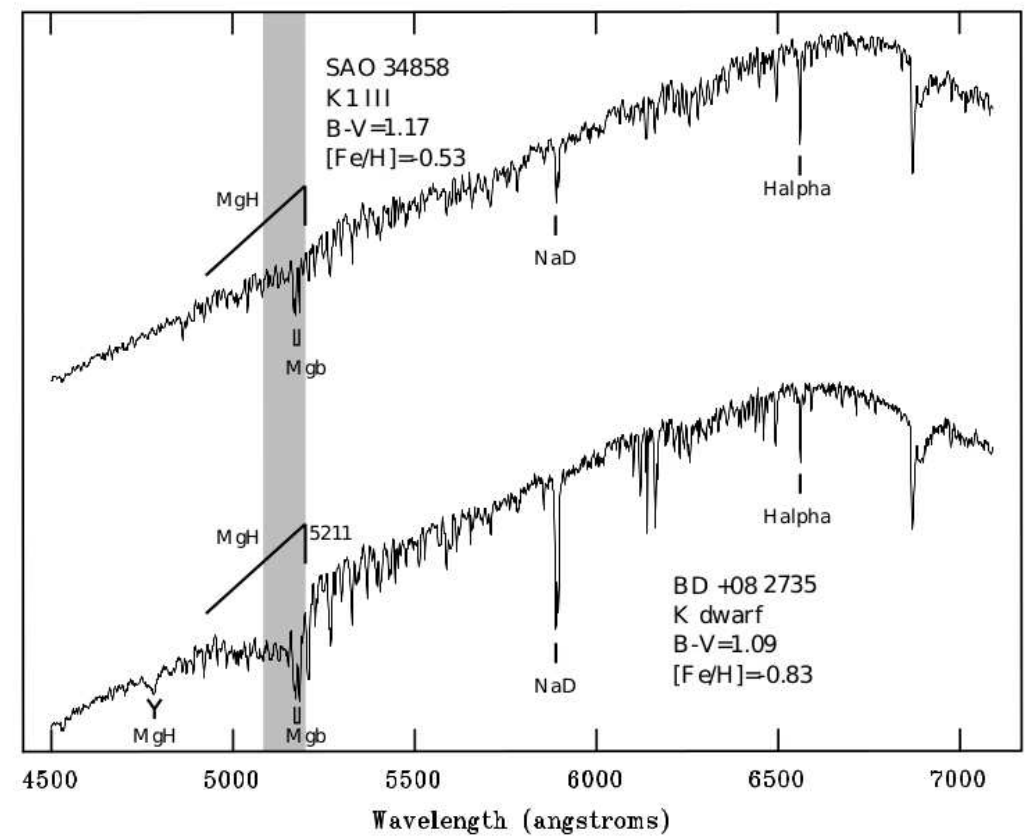

Fig. 4.1. - Spectra of a K-type giant and dwarf star. These stars have similar color and metallicity, so that differences in spectral features are the result of different gravity. Note the changes in strength of the $\mathrm{MgH}, \mathrm{Mg} b$ and $\mathrm{Na} \mathrm{D}$ features. The shaded region corresponds to the bandpass of the DDO51 filter. Figure from Majewski et al. (2000).

et al. (2001) mentions, the GGSS comprises 1302 fields (or bricks), evenly distributed on the whole sky. Bricks follow bands of constant declination separated by $5.62^{\circ}$. Within each band, bricks are separated by $5.62^{\circ}$. In addition, field centers between contiguous declination bands are shifted by $3.53^{\circ}$ along RA. As a consequence, the mean separation between bricks is $\sim 6^{\circ}$. Figure 4.3 illustrates the field positions.

Based on the expected density of targets, each GGSS field measures $\sim 0.3 \mathrm{deg}^{2}$. CCD observations were collected using three 1-m class telescopes. Fields with $\delta \leq$ $+20^{\circ}$ were observed using the 1-m Swope telescope at Las Campanas Observatory, in Chile. The remaining fields were observed with either the $0.9-\mathrm{m}$ WIYN telescope at KPNO or the $0.8-\mathrm{m}$ at McDonald Observatory. 


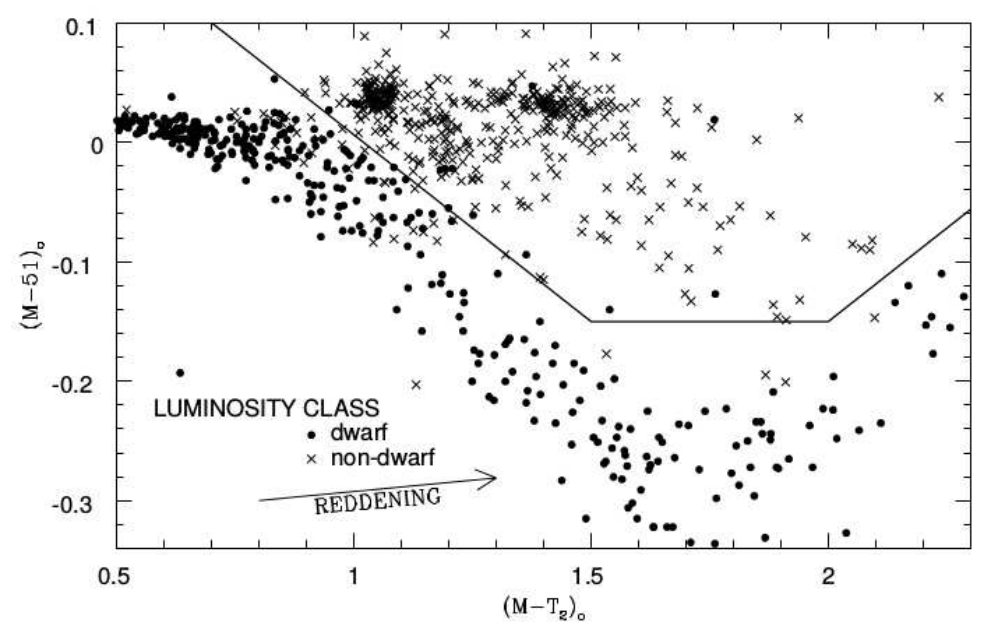

Fig. 4.2. - The $\left(M-T_{2}\right)_{o},(M-D D O 51)_{o}$ plane and the selection boundary proposed by Majewski et al. (2000) to discriminate between giants and dwarfs for $\mathrm{F}-\mathrm{K}$ stars. Within the giants, lower metallicity stars have larger $(M-D D O 51)_{o}$. Figure from Majewski et al. (2000).

\subsubsection{Low-Resolution Spectroscopic Campaign}

The GGSS team obtained medium-resolution follow-up spectroscopy of four candidate giant stars for each brick. Targets were selected with a bias towards giants showing likelihood of being lower metallicity in the brick. These spectra are intended for checking the dwarf/giant discrimination and to provide metallicities for a spectroscopic parallax. In addition, these spectra were used to derive radial velocities.

The spectroscopic data were collected using the Modular Spectrograph on the 1-m Swope telescope in Chile (though one early run was done with this instrument attached to the 2.5-m Du Pont telescope), the FOBOS spectrograph on the 1-m telescope in Fan Mountain Observatory, and the Boller \& Chivens spectrograph on the 2.3-m Bok telescope at KPNO. The FOBOS instrument (Crane et al. 2005) was specifically designed to undertake this project. The typical spectral dispersion is $\sim 1$ $\AA /$ pixel, and resolution of $4-5 \AA$. The spectrographs were set to record the $4,800-$ $6,800 \AA$ region. This spectral range is chosen to include the $\mathrm{MgH}$ and $\mathrm{Mg}$ b features 


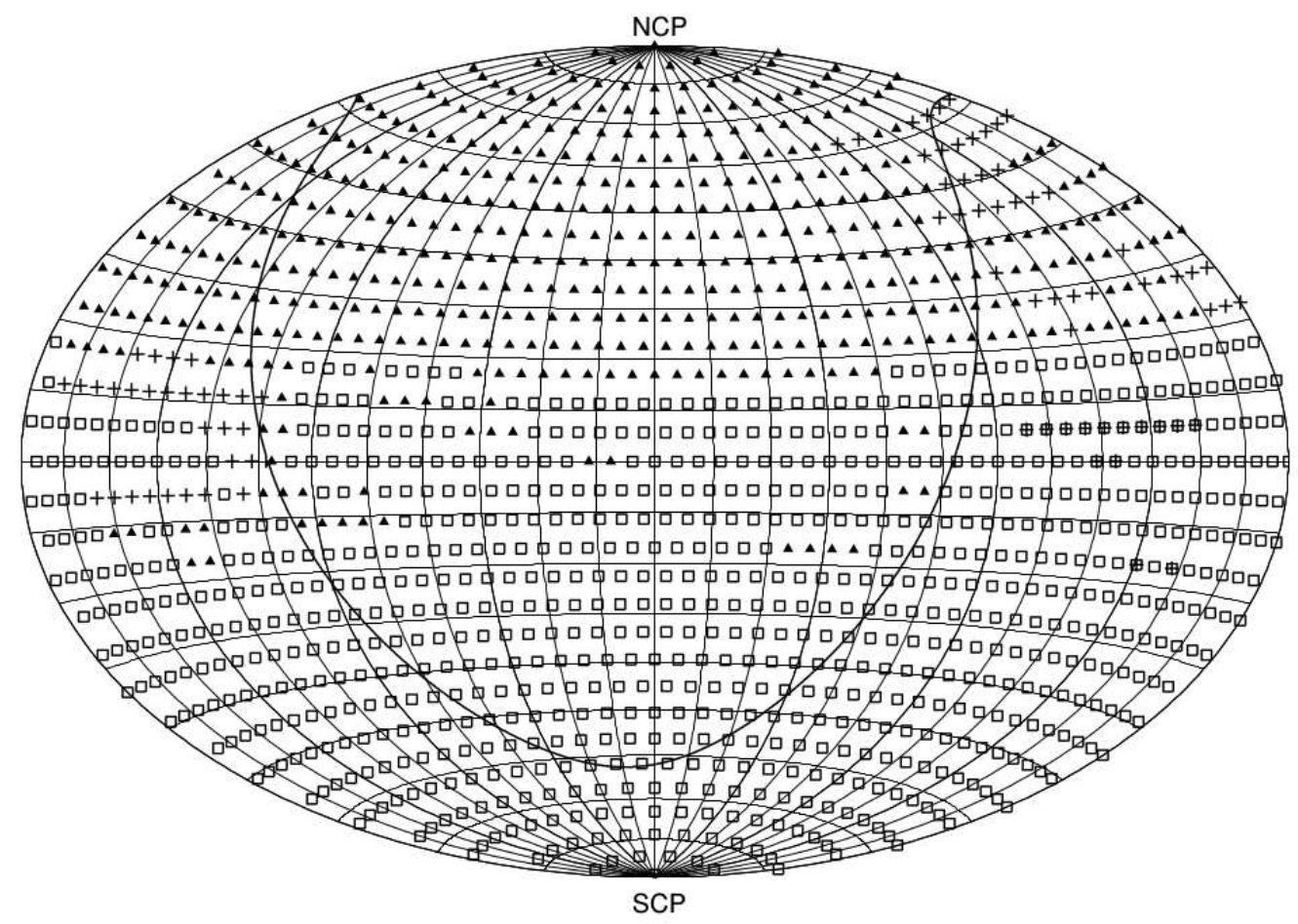

Fig. 4.3.- A Hammer-Aitoff projection of the sky in equatorial coordinates depicting the GGSS fields. The symbols indicate the telescope used to obtain the photometric data for the brick: open squares mark the Swope fields; whereas filled triangles and plus signs tag the McDonald 0.8-m and KPNO 0.9-m fields, respectively.

- sensitive to gravity - and to include features where Lick indices (Burstein et al. 1984) are defined to estimate metallicities.

\subsubsection{Radial Velocity Measurements}

Radial velocities were determined by the GGSS group using the cross-correlation technique described in Majewski et al. (2004). The method is based on the Tonry \& Davis (1979) cross-correlation technique, but addresses some particular requirements, namely: The spectra are first processed through a high-pass Fourier-filter. Because the information for the cross-correlation resides in the line slopes, a masking routine is implemented to keep only spectral lines that are expected to appear in the type 
of stars selected through the photometric criteria. The remaining portions of the spectra, which would only introduce noise to the cross-correlation, are set to zero in the mask. For each run, the cross-correlation master template is built by multiplying an observed high signal-to-noise spectrum by the mask (which is Doppler-shifted to match the RV of the observed spectrum). Majewski et al. (2004) claim that a precision of $1 / 20$ of a resolution element is achieved.

The typical uncertainty in radial velocity measurements of the GGSS stars depends on the spectrograph used. In the case of the Modular Spectrograph (on the Swope and DuPont telescopes), we find $\sim 5 \mathrm{~km} / \mathrm{s}$. Radial velocities from FOBOS can achieve a precision better than $5 \mathrm{~km} / \mathrm{s}$. This instrument is very stable as it is a fiber-fed, bench-mounted instrument. However, spectra collected with the Boller \& Chivens spectrograph (Bok) have large uncertainties. This problem arises from slit-centering errors. In this spectrograph, the pixel size of the detector is mismatched to the slit width required for stellar spectra. As a result, a Nyquist-sampled spectrum requires a slit width larger than the typical seeing disk. As a consequence, differences in slit-centering propagate as RV uncertainties.

\subsubsection{Metallicity Determination and Giant/Dwarf Discrim- ination}

We derive spectroscopic metallicities by means of two automated computer codes that yield independent metallicity estimates for the GGSS spectra. Knowledge of stellar metallicity is indispensable to derive stellar distances, due to the large impact on the color-luminosity relation for RGB stars. The GGSS did not, as a rule, derive metallicities from the GGSS spectra. Therefore, a program to systematically derive abundances from the spectra was implemented to exploit the GGSS for Galactic pop- 
ulation and dynamic studies. Both codes deliver useful parameters for giant/dwarf separation, an essential requirement for distance estimation, as the magnitude difference between the K-dwarfs and K-giants is even larger than the magnitude spread of the RGB because of metallicity.

The first code is EZ_SPAM (Easy Stellar Parameters and Metallicities) (Carlin et al. in preparation). This is a software pipeline written in IDL that determines stellar parameters, metallicities and a giant/dwarf estimation by measuring Lick spectral indices. The code measures $[\mathrm{Fe} / \mathrm{H}]$ to an accuracy of $\lesssim 0.3$ dex for spectra with signalto-noise ratio $\geq 20$, spectral resolution $1000 \lesssim R \lesssim 5000$, and spectral coverage between 4500 and 7000 A. EZ_SPAM measures the strength of 25 spectral absorption features —namely, Lick indices (Burstein et al. 1984) — and calculates [Fe/H] by interpolation in planes of index strength versus $[\mathrm{Fe} / \mathrm{H}]$, which are established by index measurements of standard stars. Even though EZ_SPAM measures many indices, it currently uses only the $\mathrm{H} \beta$, Fe and $\mathrm{Mg}$ strengths in its computations. Lick indices, as well as spectral indices in general, are a reliable and yet simple way to measure the strength of a spectral feature from spectra of moderate resolution and SNR. One of the advantages of spectral indices is that they define the continuum level around the spectral feature of interest in well-defined bandpasses [see Section 2.2]. As a result, continuum inaccuracies that arise from the spectral response of a particular spectrograph do not impact the measured indices, as long as offsets derived for the instrument are applied to each index. Such offsets are calculated from a set of standard stars observed with the same telescope and instrument configuration, and are used to place measurements from a particular instrument into EZ_SPAM's calibrated index system.

In addition to the metallicity measurements, EZ_SPAM performs a luminosity class estimate in the form of a dwarf/giant flag that ranges between 0 and 1 . This is 
accomplished by defining three estimators that take advantage of the gravity-sensitive strength of the Mg I b feature, which has been used extensively for this purpose in the past (Geisler 1984; Majewski et al. 2000). The estimators are defined in the Mg b vs. $\mathrm{H} \beta, \mathrm{Mg} \mathrm{b}$ vs. $\left(V-K_{s}\right)$, and $\mathrm{Mg} \mathrm{b}$ vs. $(\mathrm{Mg} 1+\mathrm{Mg} 2)$ planes, where giants and dwarfs reside on different regions empirically determined during EZ_SPAM development by Carlin et al. EZ_SPAM assigns a value of 0 to each estimator if a star evaluates as a dwarf, and 1 for a giant. An average of the three estimators is then reported. As a result, a star whose GIANT flag is 0 has been classified as a dwarf by the three estimators; if the flag is 1 , then the three estimators have classified it as a giant. Carlin et al. recommend setting the boundary between giants and dwarfs at GIANT $>0.5$. Using a large set of standard stars, they determined that $99 \%$ of giants stars were correctly classified as giants with this criterion. However, by adopting as a "dwarf" criterion GIANT $<0.5$, the efficiency for dwarfs is $89 \%$.

To run EZ_SPAM, the user inputs the spectra in FITS format, the measured heliocentric radial velocity and the heliocentric-to-observatory frame velocity correction that shifts the spectral features to rest frame, and, the photometric color of the stars. The latter is used to estimate the stellar effective temperature, and the code supports a variety of photometric bandpasses for this. The input colors are always internally converted into $\left(V-K_{s}\right)_{o}$ through a polynomial calibration. In our particular case, we compared metallicity results obtained with the Washington $\left(M-T_{2}\right)$ color and the 2MASS $\left(J-K_{s}\right)$ for several hundred stars. We obtained the 2MASS photometry by matching stellar coordinates from the GGSS astrometry against the PPMXL catalog of proper motions (Roeser et al. 2010). Input colors were dereddened using Schlegel extinction maps (Schlegel et al. 1998), and RJCE extinction (Majewski et al. 2011; Nidever et al. 2012). Because [Fe/H] results from EZ_SPAM were the same in all 
cases, independent of photometric system and dereddening method, we decided to run our data using 2MASS photometry and RJCE extinction only. We ran the spectra through EZ_SPAM from each telescope+instrument pair separately, because each setup requires a specific calibration offset to be applied.

The second spectral analysis code used is SP_Ace (Stellar Parameters And Chemical abundances Estimator) (Boeche \& Grebel 2016), which is a FORTRAN code that measures stellar parameters and chemical abundances from spectra with spectral resolution $R$ between 2000 and 20000, and wavelengths spanning $5212-6860 \AA$ or 8400 - 8924 A. SP_Ace works by fitting features in the whole observed spectrum via $\chi^{2}$ minimization. However, the code does not utilize a grid of synthetic spectra as other codes do, but rather a grid of polynomial functions that describe changes in the $E W$ of spectral features corresponding to each of several chemical elements as a function of $\log \mathrm{g}, T_{\text {eff }},[\mathrm{M} / \mathrm{H}]$ and element abundance. To derive parameters, the code constructs a synthetic spectrum by subtracting spectral features from a normalized continuum. These features are created from the polynomial terms that describe the behavior of the features as a function of stellar parameters and element abundance. The code then seeks the set of parameters that best match the observed spectrum.

SP_Ace input only requires normalized spectra and an estimate of the FWHM of the spectrograph. In some ways SP_Ace is more self-consistent because the code determines effective temperatures from the spectral features alone. Before running the code, we first trim the spectra to remove spectral lines with low SNR near the edge of the detectors. Next, we performed an automated normalization of the trimmed spectra by fitting low-order polynomials with IRAF's continuum task. Finally, we ran SP_Ace using these prepossessed spectra. The output of the code contains $\log g, T_{\text {eff }}$ and $[\mathrm{M} / \mathrm{H}]$ and their uncertainties. The values of these parameters correspond to the 
nominal values of the best-matching model atmosphere found in the minimization. Furthermore, the output can include abundances of up to ten chemical elements, or the user can set a flag so that SP_Ace handles the $\alpha$-elements ( $\mathrm{Mg}, \mathrm{Si}, \mathrm{Ca}$ and $\mathrm{Ti})$ as if they were a single element, and the remaining elements - except for C, N, Otogether as another single element labeled as metals. The latter is recommended by Boeche \& Grebel (2016) when the spectra have $R \sim 2000$, because it minimizes degeneracies in the parameter space when the available information in the data is limited. In this case, the output will include the parameters $[\alpha / \mathrm{H}]$ and $[\mathrm{m} / \mathrm{H}]$, instead of abundances for single elements. For our analysis, we adopt both SP_Ace metallicity estimations $[\mathrm{M} / \mathrm{H}]$ and $[\mathrm{m} / \mathrm{H}]$.

The next step in our procedure attempts to identify possible trends and offsets in the measured metallicities with respect to literature values for standards, and remove them. To accomplish this, we compared metallicity measurements - estimated with both codes - of the radial velocity standards that were observed for the GGSS calibration to sets of homogeneous metallicity measurements from literature. We applied the same trimming and normalization scheme described previously for spectra of GGSS objects. Then, we cross-matched the name IDs of the standards to the metallicity measurements available in the SIMBAD database, which also helpfully includes the bibliographic references. We sorted the references by the number of unique stars matched for each literature-telescope-spectrograph combination, and found two entries with a large number of matches, namely the studies by Prugniel et al. (2011) and Cenarro et al. (2007). We show these reference metallicities against our measurements in Figures 4.4 and 4.5, respectively, for the three metallicity estimates (EZ_SPAM's $[\mathrm{Fe} / \mathrm{H}]$, and SP_Ace's $[\mathrm{M} / \mathrm{H}]$ and $[\mathrm{m} / \mathrm{H}]$ ) that we have obtained. It is clear that the there exist offsets among our different instrumental setups. The 
standards from Swope+ModSpec show a clear trend with metallicity. Unfortunately, standards from $\mathrm{FMO}+\mathrm{FOBOS}$ and Bok+BCSpec only cover the more metal-rich end. Thus, we assume that measurements from each instrument will exhibit the same metallicity trend with respect to literature values, but exhibit independent offsets. We performed linear fits to the literature versus observed metallicity for all telescopeinstrument subsets simultaneously, with the constrain that all subsets are fitted by a single slope, but with each subset having its own zero-point. Effectively, this places our observed values onto the scale of the literature reference. We repeated this process for the data measured by EZ_SPAM and SP_Ace, and for both literature references. Finally, we apply these corrections to the GGSS targets.

As we mentioned before, both codes perform measurements of spectral features sensitive to surface gravity. EZ_SPAM delivers a dwarf/giant flag, whereasSP_Ace measures $\log g$. We combine both estimates for discrimination of giants from dwarfs. Figure 4.6 presents the distribution of $\log g$ for stars grouped as likely-dwarfs and -giants according to EZ_SPAM dwarf/giant flag.

Note to myself: Remember to write in figure for giant/dwarf separation that the contamination from the Washington-DDO51 photometry is low.

Finally, we process the quantities measured in this section for stars with multiple spectra, and merge them into a single entry per star. For EZ_SPAM results, we calculate a weighted mean for $[\mathrm{Fe} / \mathrm{H}]$ and the "GIANT" flag. We assign weights as the inverse of the uncertainty in $[\mathrm{Fe} / \mathrm{H}]$ squared. We consider stars with "GIANT" flag greater than 0.5 as giants, and dwarfs for smaller than this value. 
[Fe/H] reference: 2011Al\&A...531A.165P
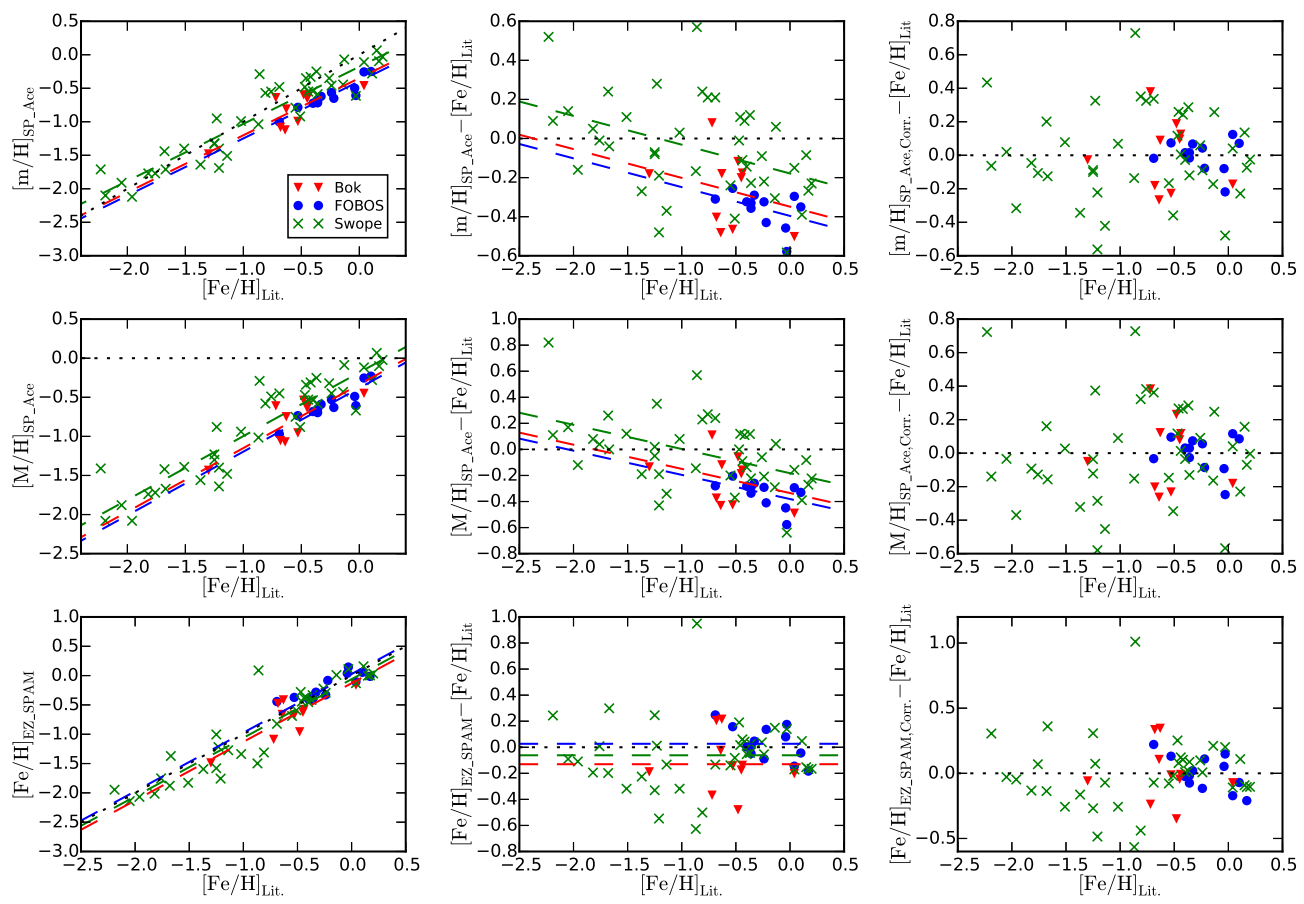

Fig. 4.4.- Calibration of spectroscopic metallicities against literature values from Prugniel et al. (2011) for standard stars. Top and middle rows show the measurements for SP_Ace's $[\mathrm{m} / \mathrm{H}]$ and $[\mathrm{M} / \mathrm{H}]$, respectively; while EZ_SPAM's $[\mathrm{Fe} / \mathrm{H}]$ is at the bottom. Panels on left and middle columns show the uncalibrated data with the linear fits. Calibrated metallicities are on the right column.

\subsubsection{Estimation of Distances}

We obtained distances to the GGSS stars by means of spectroscopic parallaxes. The basis of this technique lies in the estimation of the absolute magnitude of a star based on spectroscopically derived stellar parameters, and then calculating the stellar distance modulus according to its dereddened observed magnitude in a given bandpass. The absolute magnitude for RGB stars depends strongly on metallicity, $[\alpha / \mathrm{Fe}]$ and effective temperature. Age plays a smaller role. These parameters also affect the absolute magnitude of MS stars. To derive the stellar absolute magnitudes, we use the measured values of these parameters to select an appropriate theoretical isochrones. 
[Fe/H] reference: 2007MNRAS.374..664C
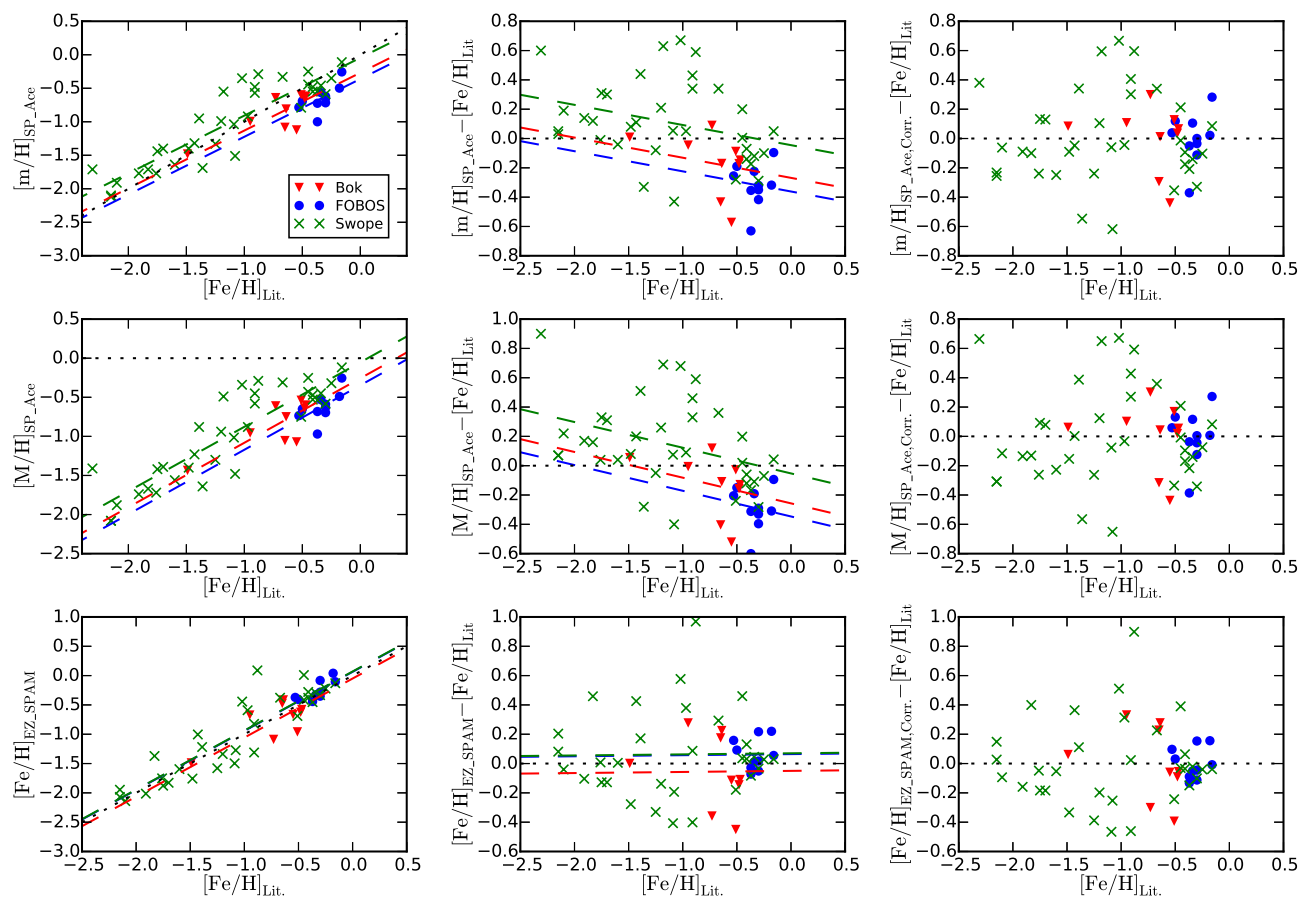

Fig. 4.5.- Same as Figure 4.4, but calibrated against measurements by Cenarro et al. (2007).

For each star, we first find the isochrone that matches the star's metallicity. In the case of SP_Ace metallicities, we also match the $[\alpha / \mathrm{Fe}]$ values. Next, we select the MS section of the isochrone if the star dwarf/giant separation identifies it as a dwarf, or the RGB section if the star is likely a giant. We adopt the absolute magnitude that corresponds to the dereddened color of the star from the isochrone section. We could have used the $T_{\text {eff }}$ estimates from SP_Ace, instead of color. However, $T_{\text {eff }}$ uncertainties are significant. This would result in larger uncertainties in absolute magnitude than that we obtain from photometric colors used as a gauge of $T_{\text {eff }}$.

In practice, isochrones are calculated for discrete values of metallicity. Furthermore, each isochrone - for a single metallicity — represents the color-magnitude plane as a set of discrete points. As a result, the derivation of absolute magnitude requires 


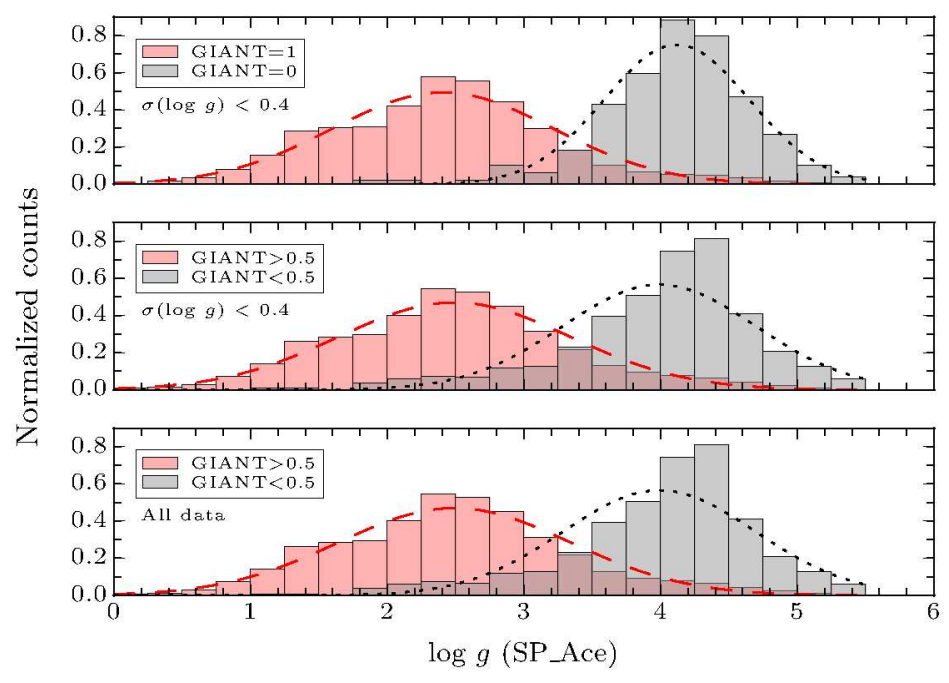

Fig. 4.6.- Giant/dwarf discrimination based on spectroscopic measurements from EZ_SPAM and SP_Ace. We classify a star as a giant (dwarf) if EZ_SPAM's GIANT $>0.5(<0.5)$ and SP_Ace $\log g \leq 3.2(\geq 3.6)$. This removes stars that can be misidentified, which potentially hinder the model minimization.

interpolation in metallicity and color. In addition, we also have to interpolate in one more parameter if we include $[\alpha / \mathrm{Fe}]$. We do these calculations with our Python code EZ_CHRONES (section 2.3.2). We prefer to use the Dartmouth isochrones set (Dotter et al. 2008). We prefer this set of isochrones because they include $[\alpha / \mathrm{Fe}]$ as a free parameter. We calculate the distances for all the GGSS stars with 11.0 Gyr isochrones, similar to Schönrich (2012), who used 11.6 Gyr isochrones as a good compromise between the probable age of the first disk stars and halo stars. For Dartmouth isochrones, the metallicity parameter spans the interval $[-2.49,+0.5]$, whereas $[\alpha / \mathrm{Fe}]$ spans $[-0.2,+0.8]$, for $[\mathrm{Fe} / \mathrm{H}] \leq-0.5$, and $[-0.2,0.2]$ for $[\mathrm{Fe} / \mathrm{H}]>-0.5$. For each star, we estimate absolute magnitudes for the six values of metallicities derived in the previous section $-[\mathrm{Fe} / \mathrm{H}],[\mathrm{M} / \mathrm{H}]$ and $[\mathrm{m} / \mathrm{H}]$ calibrated against each the Prugniel et al. (2011) and the Cenarro et al. (2007) datasets. We include the $[\alpha / \mathrm{H}]$ values for the metallicities derived with SP_Ace, but we set it to zero for $[\mathrm{Fe} / \mathrm{H}]$ values from 
EZ_SPAM. For the stars with measured $[\mathrm{Fe} / \mathrm{H}]$ or $[\alpha / \mathrm{Fe}]$ outside of the span of the Dartmouth isochrones, we set the corresponding value to be equal to the closest point in the valid $([\mathrm{Fe} / \mathrm{H}],[\alpha / \mathrm{Fe}])$ space defined by the isochrone grid. Finally, we calculate the distance using the two sets of dereddened 2MASS magnitudes (i.e., the Schlegel and the RJCE extinction values), and the six values of distance modulus. As a result, we have 12 estimates of distance for each star.

\subsection{Analysis}

In this section we derive the values of the Solar motion and Galactic rotation with respect to the Galactic frame of reference. This is accomplished by means of a model that describes the observed heliocentric velocity of stars due to their rotation around the center of the Galaxy, in addition to the peculiar motion of the Sun with respect to the velocity for the circular orbit at Solar radius. We search for the best-matching model parameters to the data by minimization of the residuals of the observed velocities against the velocities predicted by the model as a function of the parameters. In fact, we solve for two variations of the model: one for radial velocity data alone, and then for the full 3D space velocity data. The later are calculated using the radial velocity data and the proper motions matched from the PPMXL catalog. Additionally, we estimate the sensitivity of our methodology to the typical uncertainties in radial velocities and distances.

\subsubsection{Galactic Dynamical Model Description}

The purpose of our Galactic dynamical model is to find the best match to the observed velocity data for stars in the Galaxy, exploring the range of possible Milky Way kinematical parameters after adopting some simplifying assumptions regarding the 
bulk dynamical properties of the relevant stellar populations. To this end, we have built a simple and modular model that calculates the 3D and line-of-sight velocities in a heliocentric frame, for a star with coordinates $(l, b, d)$ - where $d$ is the distance to the star from the Sun - rotating around the Galactic center. The model free parameters are the Solar peculiar velocity $\left(U_{\odot}, V_{\odot}, W_{\odot}\right)$, the circular velocity of the LSR $V_{c}$, Solar distance to the Galactic center $\left(R_{o}\right)$, the rotation velocity on the Galactic plane $\left(\theta_{o}\right)$ of a single kinematical population (in this case, the disk), and a multiplicative term is a scale factor for the distance scale adopted for the dataset. We define angles, position and velocity vectors following Schönrich (2012). We illustrate them in Figure 4.7, and introduce the definitions below.

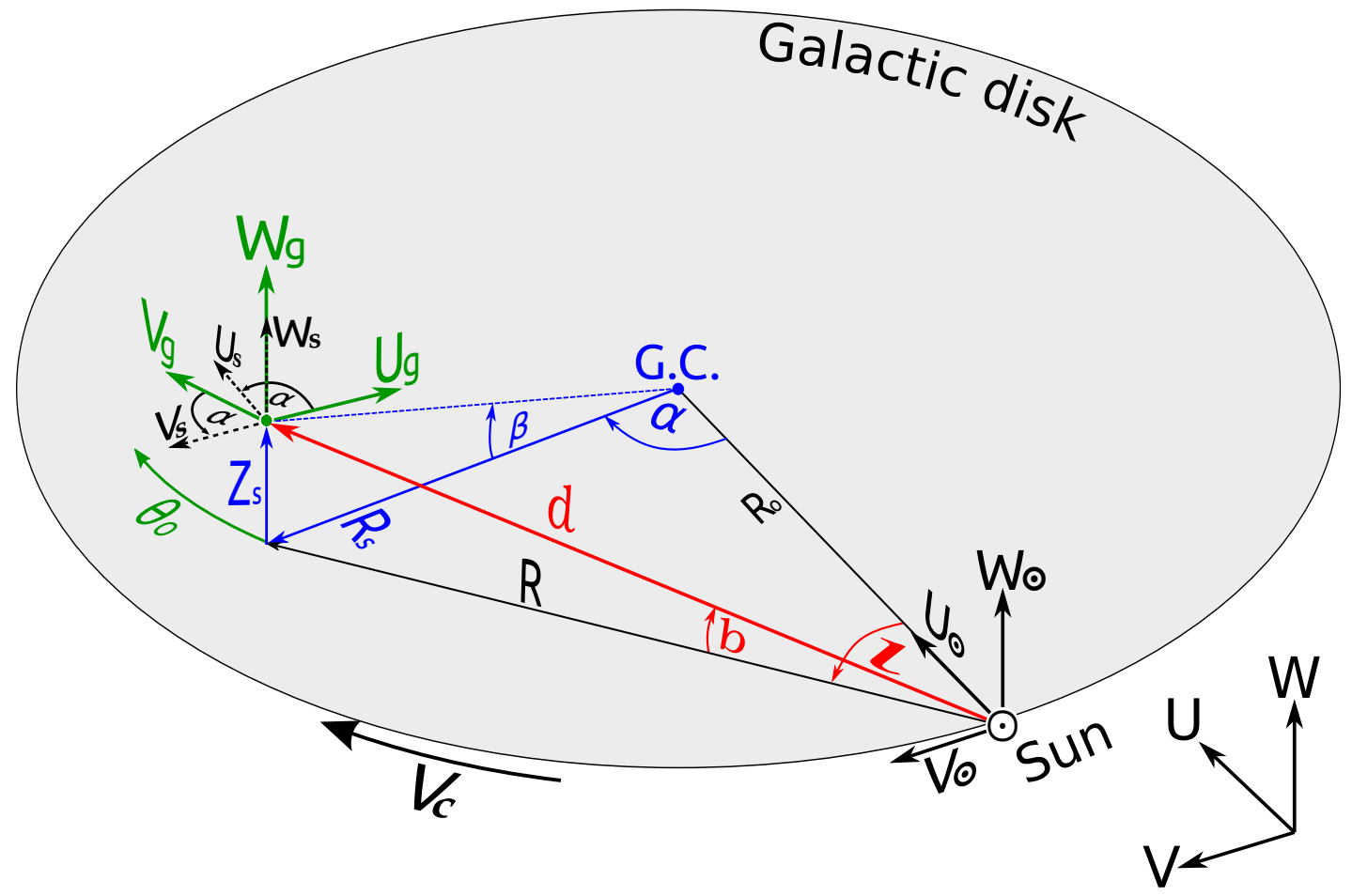

Fig. 4.7.- An illustration of the coordinates, angles and velocities defined in the model of Galactic rotation introduced in this chapter, for a star with galactic coordinates and distance $(l, b, d)$. The Sun and Galactic Center (G.C.) positions are labeled. 
First, the model performs coordinate transformations with the steps listed below:

- Convert from observed (heliocentric) Galactic longitude, latitude and distance, $\left(l, b, d_{o b s}\right)$, coordinates to the Galactocentric frame (whose origin is at the Galactic center). We first convert to a heliocentric cylindrical system $(R, Z)$,

$$
\begin{gathered}
d=d_{\text {stretch }} d_{o b s} \\
R=d \cos (b) \\
Z=d \sin (b),
\end{gathered}
$$

where $d_{\text {stretch }}$ is a multiplicative factor that scales all the distances in the model by the same amount, and allows for corrections in the estimated distances if they are systematically under- or over-estimated.

- Transform the cylindrical heliocentric into a cylindrical Galactocentric system $\left(R_{s}, Z_{s}\right)$. This transformation requires $R_{o}$ - the Sun-Galactic Center (GC hereafter) distance - assumed to be known,

$$
\begin{aligned}
& R_{s}=\left(R^{2}+R_{o}^{2}-2 R R_{o} \cos (l)\right)^{1 / 2} \\
& Z_{s}=Z
\end{aligned}
$$

- Calculate the Sun-GC-star angles $\alpha$ and $\beta$. The angle $\alpha$ is measuring the Sun-GC-star angle projected onto the Galactic plane, and increases towards the direction of Galactic rotation. The angle $\beta$ measures the component of the Sun-GC-star angle perpendicular to the Galactic plane and increases towards Galactic north. 


$$
\begin{aligned}
\cos \alpha & =\frac{R_{s}^{2}+R_{o}^{2}-R^{2}}{2 R_{s} R_{o}} \\
\sin \alpha & =\frac{R \sin (l)}{R_{s}} \\
\tan \beta & =\frac{Z_{s}}{R_{s}}
\end{aligned}
$$

Next, the model derives what the observed velocity of a model test star should be. The steps are:

- Calculate stellar velocities in the Galactocentric frame. Currently, we assume that there is a single kinematical population of stars that describes a follow a mean, mid-plane motion around the $\mathrm{GC}$ with circular (linear) velocity $\theta_{o}$. A star belonging to this population has velocity $\theta$. Velocities $\theta_{o}$ and $\theta$ point in the azimuthal direction of rotation. The magnitude of $\theta$ is modeled as

$$
\theta=\theta_{o}\left(1-f_{\theta}\left|Z_{s}\right|\right)
$$

where $f_{\theta}$ is a term that accounts for a linear decrease in $\theta$ as a function of stellar distance from the Galactic plane. This is meant to simulate the variation of mean asymmetric drift of stellar populations as a function of $Z$. In principle, Equation (4.4) can adopt any form that describes the motion of a star in the Galaxy as a function of position. We have adopted a simple axisymmetric model with a flat rotation curve corrected by $f_{\theta}$, thus $\theta$ currently is only a function of $\left|Z_{s}\right|$. Note that the model is modular in nature. Therefore, we can implement more sophisticated assumptions into Equation 4.4.

- Define the velocity components $\left(U_{g}, V_{g}, W_{g}\right)$ for a star, similar to the $(U, V, W)$ components that describe the Solar motion in the Galactic Standard of Rest 
(GSR, hereafter), such that $U_{g}$ increases towards the Galactic Center, $V_{g}$ increases in the direction of rotation, and $W_{g}$ increases towards Galactic north pole but defined at the position of the star rather than the Sun. Then the rotation model results in:

$$
\left(U_{g}, V_{g}, W_{g}\right)=(0, \theta, 0)
$$

- Convert back to Galactic frame. The 3-D $(U, V, W)$ space velocity for a star, defined at Sun's position, is:

$$
\begin{aligned}
& U_{s}=U_{g} \cos \alpha+V_{g} \sin \alpha \\
& V_{s}=-U_{g} \sin \alpha+V_{g} \cos \alpha \\
& W_{s}=W_{g}
\end{aligned}
$$

These velocities are now in the GSR. To convert into the heliocentric frame, we need to correct by the Solar motion with respect to the GSR frame. For the Sun, these velocities are $(U, V, W)=\left(U_{\odot}, V_{\odot}+V_{c}, W_{\odot}\right) . V_{c}$ is the velocity of the circular orbit at Solar radius $R_{o}$, and $\left(U_{\odot}, V_{\odot}, W_{\odot}\right)$ the velocity components with respect to the velocity of the circular orbit. Thus, the $3 \mathrm{D}$-velocity for a star in the heliocentric frame is:

$$
\begin{aligned}
& U_{h}=U_{s}-U_{\odot} \\
& V_{h}=V_{s}-\left(V_{c}+V_{\odot}\right) \\
& W_{h}=W_{s}-W_{\odot}
\end{aligned}
$$

If our data comprise 3D velocities derived from radial velocities and proper motions, then one would minimize the model with respect to Equation 4.7 and derive 
the model parameters. If we want to only minimize against radial velocities, then we would use Equations 4.6 and the definition of the dot product as follows to obtain the line-of-sight velocity:

- In the Galactic frame, a star has $(X, Y, Z)$ coordinates $\left(X_{s}, Y_{s}, Z_{s}\right)$. Because the inner product can be expressed as

$$
\begin{aligned}
& \vec{r} \cdot \vec{v}=X_{s} U_{s}+Y_{s} V_{s}+Z_{s} W_{s} \\
& \vec{r} \cdot \vec{v}=|\vec{r}||\vec{v}| \cos \gamma,
\end{aligned}
$$

we can solve for the projection of $\vec{v}$ along the radial vector $\vec{r}$. Thus,

$$
V_{G S R}=\frac{X_{s} U_{s}+Y_{s} V_{s}+Z_{s} W_{s}}{\left(X_{s}^{2}+Y_{s}^{2}+Z_{s}^{2}\right)^{\frac{1}{2}}}
$$

- Finally, we calculate a line-of-sight velocity correction for the Solar motion that will be subtracted from Equation (4.9),

$$
V_{\text {corr }}=U_{\odot} \cos l \cos b+\left(V_{c}+V_{\odot}\right) \sin l \cos b+W_{\odot} \sin b
$$

to find that the line-of-sight velocity in the heliocentric frame is:

$$
V_{H E L I O}=V_{G S R}-V_{\text {corr }}
$$

\subsubsection{Model Minimization and Results}

We solve for the best matching parameters of the model by least-squares minimization, using the LMFIT routine in Python. The free parameters to fit for the model are $\theta_{o}, f_{\theta}, V_{c}, U_{\odot}, V_{\odot}, W_{\odot}$. Because we calibrated our metallicities against metallicity 
standards, we set $d_{\text {stretch }}=1$ as a fixed parameter. For the minimization, we use the Levenberg-Marquardt and the Nelder-Mead algorithms. We fit the model to two sets of data: line-of-sight (radial) velocities from the GGSS only, and 3D space velocities calculated from the GGSS radial velocities in combination with proper motions from the PPMXL catalog.

First, to fit line-of-sight velocities, we minimize the residuals for the $N$ stars in the dataset given by:

$$
\text { residuals }=\sum_{i}^{N} w_{i}\left(V_{H E L I O, M O D E L, i}-V_{H E L I O, G G S S, i}\right)
$$

where $V_{H E L I O, M O D E L, i}$ corresponds to Equation 4.11. For the weight $w_{i}$ for the i-th star we adopt three different forms. First, we can set them equal for all stars, and do an unweighted fit. The other two forms set $w_{i}=\frac{1}{\sigma_{i}^{2}}$, where $\sigma$ equals the uncertainty in the radial velocity measurement or uncertainty in distance. However, we find that the unweighted fit produces results that are more consistent among the different distance estimates corresponding to the different metallicity measurements. Likely this is because while we know the overall uncertainties in the GGSS in a statistical fashion, they are not accurate enough on a star-by-star basis.

Similarly, to fit 3D space velocities, we minimize the residuals for the $N$ stars in the dataset given by:

$$
\begin{array}{r}
\text { residuals }=\sum_{i}^{N}\left[w_{U, i}\left(U_{h, M O D E L, i}-U_{h, G G S S, i}\right)+\right. \\
w_{V, i}\left(V_{h, M O D E L, i}-V_{h, G G S S, i}\right)+ \\
\left.w_{W, i}\left(W_{h, M O D E L, i}-W_{h, G G S S, i}\right)\right],
\end{array}
$$

where - for the i-th star- $\left(U_{h, M O D E L, i}, V_{h, M O D E L, i}, W_{h, M O D E L, i}\right)$ are the $3 \mathrm{D}$ velocity 
components defined in Equation 4.7, whereas $\left(U_{h, G G S S, i}, V_{h, G G S S, i}, W_{h, G G S S, i}\right)$ are the observed $3 \mathrm{D}$ velocity components in the heliocentric frame. The terms $\left(w_{U, i}, w_{V, i}, w_{W, i}\right)$ define the weight applied for the $\left(U_{i}, V_{i}, W_{i}\right)$ components, respectively. Nevertheless, we apply equal weights to all velocities.

We run the minimization for line-of-sight (l.o.s.) and 3D velocities multiple times. For each of these "runs" we use subsets of data selected after applying a particular selection in $(l, b, d)$.

In all "runs", we reject stars with $\left|V_{\text {helio }}\right|>300 \mathrm{~km} / \mathrm{s}$ and $\left(U^{2}+V^{2}+W^{2}\right)^{\frac{1}{2}}>$ $300 \mathrm{~km} / \mathrm{s}$, for l.o.s. and 3D velocities, respectively. These rejected high-velocity stars likely have streaming motions that differ considerably from the circular motions that we model. As a consequence, both subsets do not contain the same number of stars. Furthermore, we have rejected stars that have an ambiguous dwarf/giant classification. For each "run", we minimize the residuals twelve times because this is the number of distance estimates available, based on every combination of the three metallicity estimates, two metallicity calibrations, and two dereddening estimates. The results are shown in Tables 4.3 and 4.4, where we present the median values for model parameters for the "runs", using l.o.s. and 3D velocities, respectively. Moreover, Tables 4.1 and 4.2 show results for a single "run" using l.o.s. and 3D velocities, respectively, for the data subset of stars from the whole sky, except for $|l|<25^{\circ}$

Perhaps one of the interesting results found is a large $U_{\odot}$ component for the l.o.s. "run" of the whole set, reaching values of $19 \mathrm{~km} / \mathrm{s}$. There are two possibilities that may explain this outcome. First, stars in our sample towards this part of the Galaxy have circular motion around the GC, but our assumption of a flat rotation curve represented by $\theta$ in our model - breaks down for small Galactic radius $R$. It is known 
empirically that the rotation curve for disk galaxies increases almost linearly at small radii. In addition, the azimuthal motion of stars at low Galactic longitude produces a large signal into the $U_{h}$ component. A second option is that we have detected stars with non-circular motion, likely from a dynamical substructure. For example, the long Galactic Bar reaches as far as $l \sim 25^{\circ}$, and has a half-length of $5.0 \pm 0.2 \mathrm{kpc}$ (Wegg et al. 2015). Stars in the bar will exhibit large $\left|U_{h}\right|$ motions from streaming motions. In addition, the GGSS may contain stars from tidal streams, which will have kinematics different than that of the disk. By instance, Majewski et al. (2012) used the GGSS to find extended tidal debris associated with $\omega$ Centauri. Even though we have rejected stars with velocities extreme velocities $( \pm 300 \mathrm{~km} / \mathrm{s})$, we still may have stars belonging to substructures that can follow non-disk motions.

Although results for most model parameters show scatter for different runs, it is clear that some of them are more stable than others for the different subsets. There are some possible explanations for this. First, data subsets used in the runs are located on different regions of the sky, and subsequently, have different dynamical properties. Second, we chose these subsets purposefully to test how they impact the different parameters. For example, $U_{\odot}$ is more sensitive to l.o.s. velocity data of stars close to the Galactic equator and longitudes close to the Galactic center and anti-center. In contrast, $W_{\odot}$ is more sensitive to l.o.s. velocity measurements for stars located towards the Galactic poles. We carried out a series of tests to assess the uncertainty of the results as a function of radial velocity and distance uncertainties. The implementation and results of such a test are presented in the next section. 
Table 4.1. Fit results for minimization of line-of-sight velocity residuals, unweighted, for a single run. The data subset corresponds to the whole GGSS sample, except stars with $|l|<25 \mathrm{deg}$. The "Distance origin" column provides details about the distance derivation for the subsample of stars, namely the "metallicity origin", "dereddening" (RJCE or Schlegel maps), and "literature source for metallicity calibration" ("1" for Prugniel et al. 2011 and "2" for Cenarro et al. 2007).

\begin{tabular}{lccccccc}
\hline \hline Distance origin & $N_{\text {stars }}$ & $\begin{array}{c}U_{\odot} \\
{[\mathrm{km} / \mathrm{s}]}\end{array}$ & $\begin{array}{c}W_{\odot} \\
{[\mathrm{km} / \mathrm{s}]}\end{array}$ & $\begin{array}{c}V_{c}+V_{\odot} \\
{[\mathrm{km} / \mathrm{s}]}\end{array}$ & $\begin{array}{c}\theta_{o} \\
{[\mathrm{~km} / \mathrm{s}]}\end{array}$ & $\begin{array}{c}V_{c}+V_{\odot}-\theta_{o} \\
{[\mathrm{~km} / \mathrm{s}]}\end{array}$ & $\theta_{f}$ \\
\hline$[F e / H]$, RJCE, 1 & 3584 & 14.3 & 6.7 & 209.3 & 189.2 & 20.1 & 0.15 \\
{$[M / H]$, RJCE, 1} & 3584 & 14.6 & 6.1 & 196.3 & 175.6 & 20.8 & 0.14 \\
{$[m / H]$, RJCE, 1} & 3567 & 14.5 & 6.1 & 200.1 & 179.7 & 20.4 & 0.14 \\
{$[\mathrm{Fe} / \mathrm{H}], \mathrm{RJCE}, 2$} & 3584 & 14.2 & 6.7 & 199.2 & 179.1 & 20.1 & 0.15 \\
{$[\mathrm{M} / \mathrm{H}], \mathrm{RJCE}, 2$} & 3584 & 14.5 & 6.1 & 191.6 & 170.8 & 20.8 & 0.14 \\
{$[\mathrm{~m} / \mathrm{H}], \mathrm{RJCE}, 2$} & 3567 & 14.4 & 6.1 & 194.6 & 174.2 & 20.4 & 0.14 \\
{$[\mathrm{Fe} / \mathrm{H}], \mathrm{Sch}+, 1$} & 3584 & 13.4 & 6.4 & 211.2 & 197.4 & 13.8 & 0.19 \\
{$[\mathrm{M} / \mathrm{H}], \mathrm{Sch}+, 1$} & 3584 & 14.0 & 5.7 & 190.3 & 174.9 & 15.4 & 0.18 \\
{$[\mathrm{~m} / \mathrm{H}], \mathrm{Sch}+, 1$} & 3567 & 13.9 & 5.6 & 193.3 & 178.1 & 15.2 & 0.18 \\
{$[\mathrm{Fe} / \mathrm{H}], \mathrm{Sch}+, 2$} & 3584 & 13.2 & 6.4 & 202.1 & 188.1 & 14.0 & 0.18 \\
{$[\mathrm{M} / \mathrm{H}], \mathrm{Sch}+, 2$} & 3583 & 13.8 & 5.7 & 186.7 & 171.3 & 15.4 & 0.18 \\
{$[\mathrm{~m} / \mathrm{H}], \mathrm{Sch}+, 2$} & 3567 & 13.8 & 5.6 & 189.1 & 173.9 & 15.1 & 0.18 \\
\hline
\end{tabular}

Table 4.2. Same as table 4.1, but for minimization of 3d velocity residuals, unweighted. Whole GGSS sample, except stars with $|b|<25$ deg.

\begin{tabular}{lccccccc}
\hline \hline Metallicity origin & $N_{\text {stars }}$ & $U_{\odot}$ & $\begin{array}{c}V_{c}+V_{\odot} \\
{[\mathrm{km} / \mathrm{s}]}\end{array}$ & $W_{\odot}$ & $\begin{array}{c}\theta_{o} \\
{[\mathrm{~km} / \mathrm{s}]}\end{array}$ & $\begin{array}{c}V_{c}+V_{\odot}-\theta_{o} \\
{[\mathrm{~km} / \mathrm{s}]}\end{array}$ & $\theta_{f}$ \\
\hline$[\mathrm{Fe} / \mathrm{H}], \mathrm{RJCE}, 1$ & 3453 & 11.59 & 216.4 & 6.81 & 201.7 & 14.7 & 0.14 \\
{$[\mathrm{M} / \mathrm{H}], \mathrm{RJCE}, 1$} & 3457 & 11.58 & 208.7 & 6.84 & 192.8 & 16.0 & 0.13 \\
{$[\mathrm{~m} / \mathrm{H}], \mathrm{RJCE}, 1$} & 3438 & 11.49 & 206.6 & 6.94 & 191.0 & 15.5 & 0.13 \\
{$[\mathrm{Fe} / \mathrm{H}], \mathrm{RJCE}, 2$} & 3436 & 11.9 & 213.9 & 7.1 & 199.1 & 14.8 & 0.14 \\
{$[\mathrm{M} / \mathrm{H}], \mathrm{RJCE}, 2$} & 3439 & 11.84 & 207.6 & 6.91 & 191.2 & 16.3 & 0.12 \\
{$[\mathrm{~m} / \mathrm{H}], \mathrm{RJCE}, 2$} & 3425 & 11.87 & 207.9 & 6.89 & 192.1 & 15.8 & 0.13 \\
{$[\mathrm{Fe} / \mathrm{H}], \mathrm{Sch}+, 1$} & 3400 & 12.42 & 204.3 & 6.91 & 192.1 & 12.2 & 0.16 \\
{$[\mathrm{M} / \mathrm{H}], \mathrm{Sch}+1$} & 3407 & 13.01 & 196.3 & 7.3 & 181.9 & 14.4 & 0.15 \\
{$[\mathrm{~m} / \mathrm{H}], \mathrm{Sch}+, 1$} & 3397 & 12.98 & 195.8 & 7.21 & 181.8 & 14.0 & 0.15 \\
{$[\mathrm{Fe} / \mathrm{H}], \mathrm{Sch}+, 2$} & 3379 & 13.15 & 200.6 & 6.93 & 188.1 & 12.6 & 0.16 \\
{$[\mathrm{M} / \mathrm{H}], \mathrm{Sch}+, 2$} & 3395 & 12.98 & 193.8 & 7.49 & 179.5 & 14.3 & 0.15 \\
{$[\mathrm{~m} / \mathrm{H}], \mathrm{Sch}+, 2$} & 3389 & 13.08 & 194.1 & 7.26 & 179.8 & 14.2 & 0.15 \\
\hline
\end{tabular}


Table 4.3. Results for derived parameters from different "runs" —i.e. different data subsets - , obtained for minimization of line-of-sight velocity residuals, unweighted. Each column shows the median value and standard deviation for the 12 distance estimations in each run of the model.

\begin{tabular}{lccccccc}
\hline \hline \multicolumn{1}{c}{ Subset } & $N_{\text {stars }}$ & $\begin{array}{c}U_{\odot} \\
{[\mathrm{km} / \mathrm{s}]}\end{array}$ & $\begin{array}{c}V_{c}+V_{\odot} \\
{[\mathrm{km} / \mathrm{s}]}\end{array}$ & $\begin{array}{c}W_{\odot} \\
{[\mathrm{km} / \mathrm{s}]}\end{array}$ & $\begin{array}{c}\theta_{o} \\
{[\mathrm{~km} / \mathrm{s}]}\end{array}$ & $\begin{array}{c}V_{c}+V_{\odot}-\theta_{o} \\
{[\mathrm{~km} / \mathrm{s}]}\end{array}$ & $\theta_{f}$ \\
\hline Whole set & 4224 & $19.0 \pm 0.2$ & $178.4 \pm 13.6$ & $4.4 \pm 0.4$ & $158.4 \pm 11.0$ & $17.4 \pm 4.2$ & $0.190 \pm 0.04$ \\
$|l|>25^{\circ}$ & 3581 & $14.8 \pm 0.4$ & $189.5 \pm 7.3$ & $6.6 \pm 0.5$ & $170.4 \pm 4.3$ & $18.0 \pm 3.8$ & $0.165 \pm 0.02$ \\
$|l|>25^{\circ} \wedge b>0^{\circ}$ & 1640 & $12.3 \pm 0.6$ & $226.9 \pm 9.2$ & $4.5 \pm 2.0$ & $206.8 \pm 8.1$ & $17.6 \pm 4.3$ & $0.150 \pm 0.04$ \\
$|l|>25^{\circ} \wedge b<0^{\circ}$ & 1941 & $15.7 \pm 0.8$ & $167.2 \pm 8.2$ & $7.8 \pm 1.1$ & $145.5 \pm 5.5$ & $19.2 \pm 3.4$ & $0.170 \pm 0.03$ \\
$|l|>25^{\circ} \wedge|b|<25^{\circ}$ & 1583 & $13.7 \pm 0.6$ & $218.8 \pm 11.0$ & $6.6 \pm 1.1$ & $203.3 \pm 12.2$ & $13.7 \pm 2.6$ & $0.195 \pm 0.02$ \\
\hline
\end{tabular}

Table 4.4. Same as Table 4.3, but for minimization of 3d velocity residuals, unweighted.

\begin{tabular}{lccccccc}
\hline \hline \multicolumn{1}{c}{ Subset } & $N_{\text {stars }}$ & $\begin{array}{c}U_{\odot} \\
{[\mathrm{km} / \mathrm{s}]}\end{array}$ & $\begin{array}{c}V_{c}+V_{\odot} \\
{[\mathrm{km} / \mathrm{s}]}\end{array}$ & $\begin{array}{c}W_{\odot} \\
{[\mathrm{km} / \mathrm{s}]}\end{array}$ & $\begin{array}{c}\theta_{o} \\
{[\mathrm{~km} / \mathrm{s}]}\end{array}$ & $\begin{array}{c}V_{c}+V_{\odot}-\theta_{o} \\
{[\mathrm{~km} / \mathrm{s}]}\end{array}$ & $\theta_{f}$ \\
\hline Whole set & 4004 & $14.1 \pm 0.5$ & $201.3 \pm 9.5$ & $7.0 \pm 0.5$ & $185.9 \pm 8.4$ & $15.6 \pm 1.1$ & $0.145 \pm 0.01$ \\
$|l|>25^{\circ}$ & 3416 & $12.2 \pm 0.9$ & $205.4 \pm 9.9$ & $6.9 \pm 0.2$ & $191.1 \pm 8.2$ & $14.6 \pm 1.2$ & $0.145 \pm 0.01$ \\
$|l|>25^{\circ} \wedge b>0^{\circ}$ & 1553 & $14.1 \pm 0.9$ & $216.0 \pm 15.3$ & $6.1 \pm 1.0$ & $202.1 \pm 14.8$ & $14.2 \pm 1.0$ & $0.150 \pm 0.01$ \\
$|l|>25^{\circ} \wedge b<0^{\circ}$ & 1862 & $10.8 \pm 0.8$ & $191.7 \pm 3.6$ & $7.9 \pm 0.3$ & $176.4 \pm 3.6$ & $14.7 \pm 1.6$ & $0.140 \pm 0.01$ \\
$|l|>25^{\circ} \wedge|b|<25^{\circ}$ & 1493 & $12.1 \pm 1.6$ & $205.4 \pm 13.6$ & $4.1 \pm 0.6$ & $191.9 \pm 12.9$ & $13.8 \pm 0.8$ & $0.125 \pm 0.01$ \\
\hline
\end{tabular}




\subsubsection{Model Tolerance to Distance and Radial Velocity Un- certainties}

In this section we estimate how measurement uncertainties in the GGSS dataset impact the parameters that we derived through residual minimization of l.o.s. velocities. To do so, we fix the parameters of the model that we solved for in the previous section. We set $\left(U_{\odot}, V_{c}+V_{\odot}, W_{\odot}\right)=(14,238+12,6.5) \mathrm{km} / \mathrm{s}, R_{o}=8.27 \mathrm{kpc}($ Schönrich 2012), $\theta_{o}=205 \mathrm{~km} / \mathrm{s}, \theta_{f}=0.1$ and $d_{\text {stretch }}=1$. For any GGSS star, distance and radial velocity have significant uncertainties, while sky coordinates can be considered exact quantities. Thus, we generate a set of "true" radial velocities by evaluating the model for the whole GGSS sample using the measured GGSS distances — which for now we adopt as the "true" distances - together with the fixed parameters. Next, we draw a set of synthetic "observed" radial velocities and distances, as the sum of the "true" values and noise. The noise applied to distance and velocity is drawn randomly from normal distributions with sigmas $\left(\sigma(\right.$ dist $\left.), \sigma\left(V_{\text {helio }}\right)\right)$, respectively. Then, we solve the model parameters using the synthetic distances and radial velocities as if they were "observed" data. We repeated this process 1000 times. Finally, we repeated the whole process for a set of values in both $\left(\sigma(\right.$ dist $)$ and $\left.\sigma\left(V_{\text {helio }}\right)\right)$.

The results of this test are shown in Figures 4.8-4.13, and they give a clear view on how l.o.s. velocities and distance uncertainties affect the parameters that we want to solve for with our model. We estimate the GGSS radial velocities to have uncertainties of at least $5 \mathrm{~km} \mathrm{~s}^{-1}$, although the measurements for spectra from the Bok telescope have much larger uncertainties, up to $30 \mathrm{~km} / \mathrm{s}$. Additionally, distance errors are expected to be large due to the uncertainties in metallicity and dereddening uncertainties. 


\subsection{Conclusions and Future Work}

We have presented preliminary results for the Solar motion and the LSR velocity derived using the Grid Giant Star Survey and a simple model of Galactic rotation. We derived stellar metallicities from the GGSS spectra, and combined these information with photometry to derive distances through isochrones. We find that the results for $U_{\odot}$ and $W_{\odot}$ are stable (scatter of a few $\mathrm{km} \mathrm{s}^{-1}$ ) for the different distance estimations that we use for the model minimization. For a sample that does not include stars towards the Galactic center, we find evidence for $U_{\odot} \sim 14 \mathrm{~km} \mathrm{~s}^{-1}$, which is in agreement with the values found by Schönrich (2012) and Francis \& Anderson (2014). This value of $U_{\odot}$ is high compared to other estimations in the literature. For example, Dehnen \& Binney (1998) derived $U_{\odot}=10.0$, while Francis \& Anderson (2009) measured $7.5 \mathrm{~km} \mathrm{~s}^{-1}$. However, the $U_{\odot}$ value is considerably higher when we include stars towards the Galactic center in the sample. In addition, results for $W_{\odot}=$ also agree with literature values.

On the contrary, results for circular velocity $V_{c}, \theta_{o}$, and $V_{\odot}$ exhibit large fluctuations and uncertainties. This motivates the implementation of a simulation to assess the effects of measurement uncertainties in the set of derived parameters. We find a similar behavior between the observed parameters and the simulations. Uncertainties in the GGSS have a small impact on $U_{\odot}$ and $W_{\odot}$, but a large impact on $V_{c}, \theta_{o}$, and $V_{\odot}$. Therefore, our preliminary results should be valid for $U_{\odot}$ and $W_{\odot}$ only. We note that the velocity of the Sun with respect to the reference population, measured from $V_{\odot}+V_{c}-\theta_{o}$ is also a stable parameter for the different distance estimations.

The design of the model allows implementations of more complex assumptions. As part of our future work, we plan to model the effect of metallicity in the rotation $\theta_{o}$ and properly account for different (disk and halo) populations observed in the GGSS. 
In addition, we plan to exploit the GGSS to characterize the chemistry (metallicity and $[\alpha / F e])$ for the halo and the disk. 


\section{$U_{\odot}[\mathrm{km} / \mathrm{s}]$}
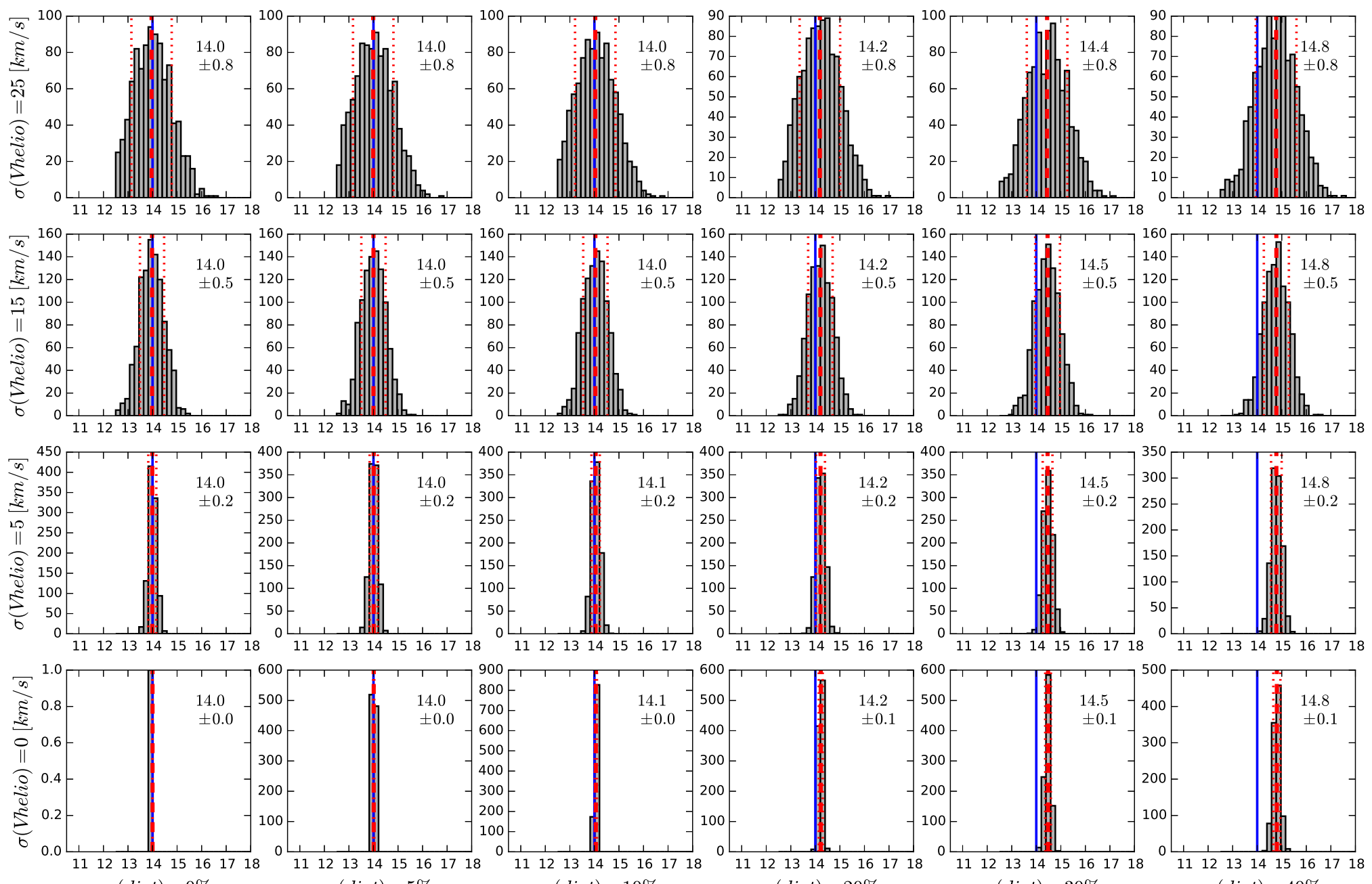

Fig. 4.8.- Simulations of the impact of uncertainties in distance and l.o.s. velocity in the measured $U_{\odot}$, for a range of uncertainty amounts. The blue line indicates the input value for the model with no noise $\left(U_{\odot}=14 \mathrm{~km} / \mathrm{s}\right)$, while the red dashed and red dotted lines show the median and standard deviation of the results. 


\section{$W_{\odot}[\mathrm{km} / \mathrm{s}]$}
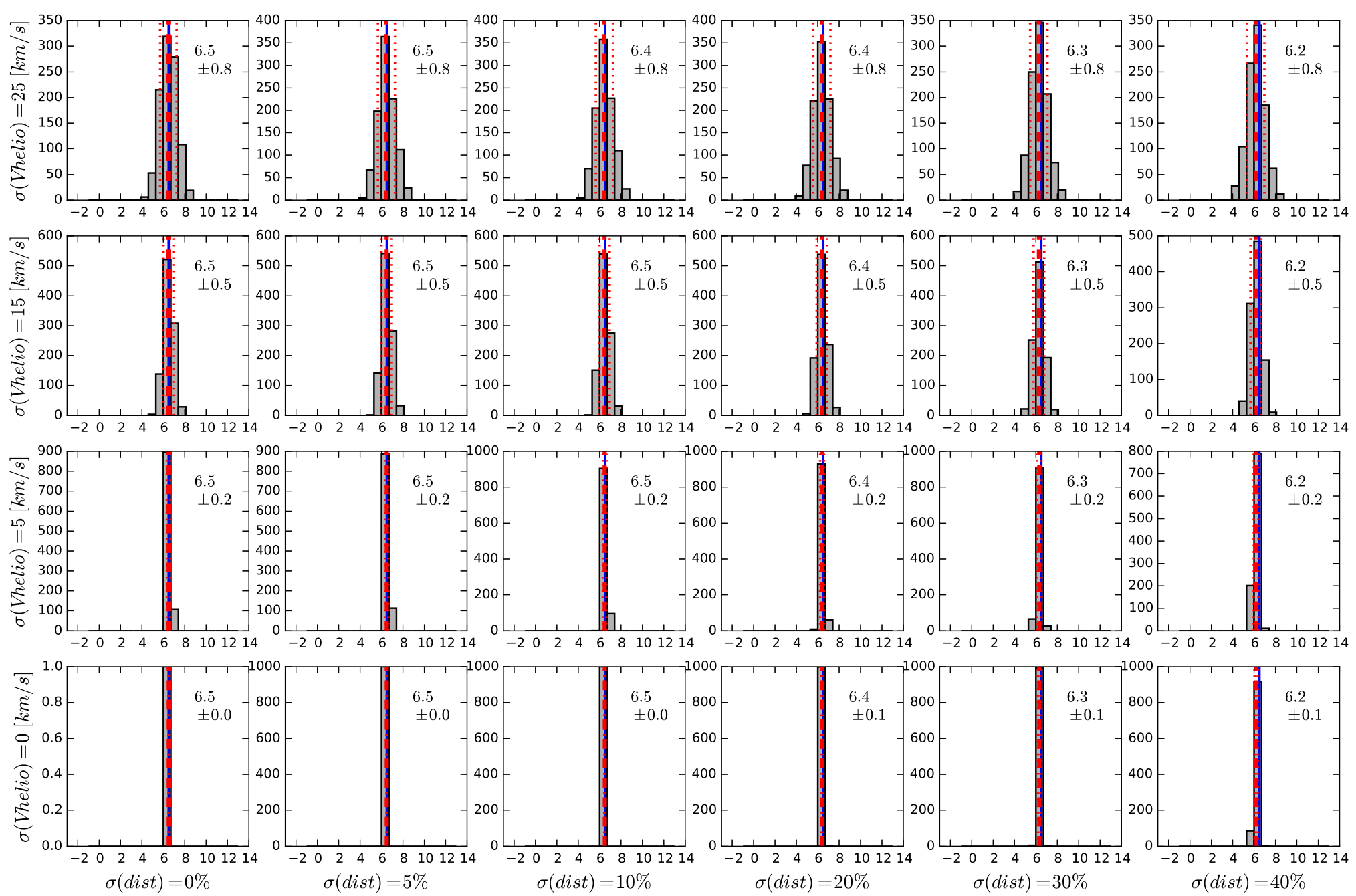

Fig. 4.9.- Same as Figure 4.8, for $W_{\odot}$. Initial value is $W_{\odot}=6.5 \mathrm{~km} / \mathrm{s}$. 


\section{$V_{c}+V_{\odot}[\mathrm{km} / \mathrm{s}]$}
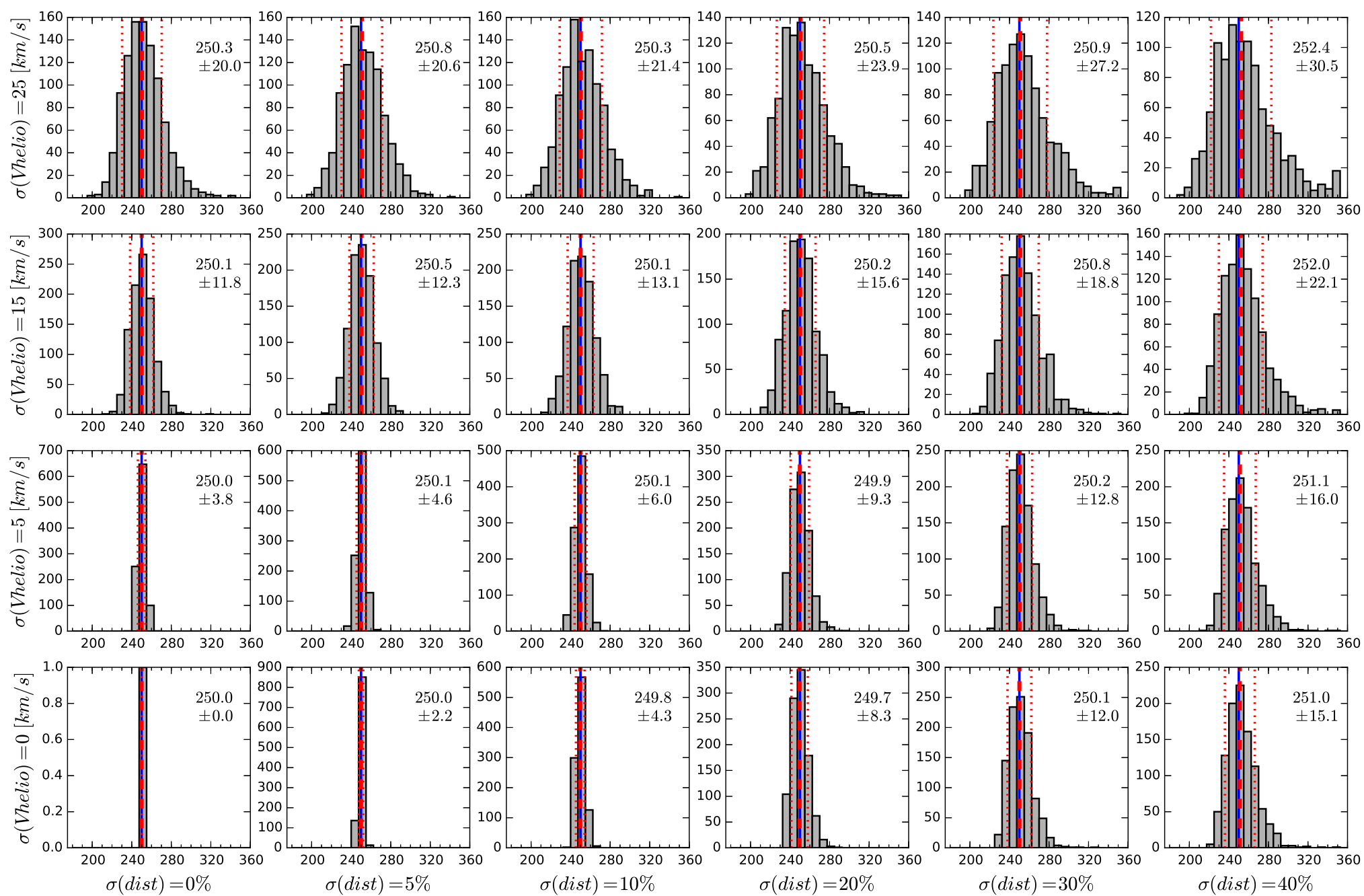

Fig. 4.10.- Same as Figure 4.8, for $V_{c}+V_{\odot}$. Initial value is $V_{c}+V_{\odot}=250 \mathrm{~km} / \mathrm{s}$. 


\section{$\theta_{o}[\mathrm{~km} / \mathrm{s}]$}
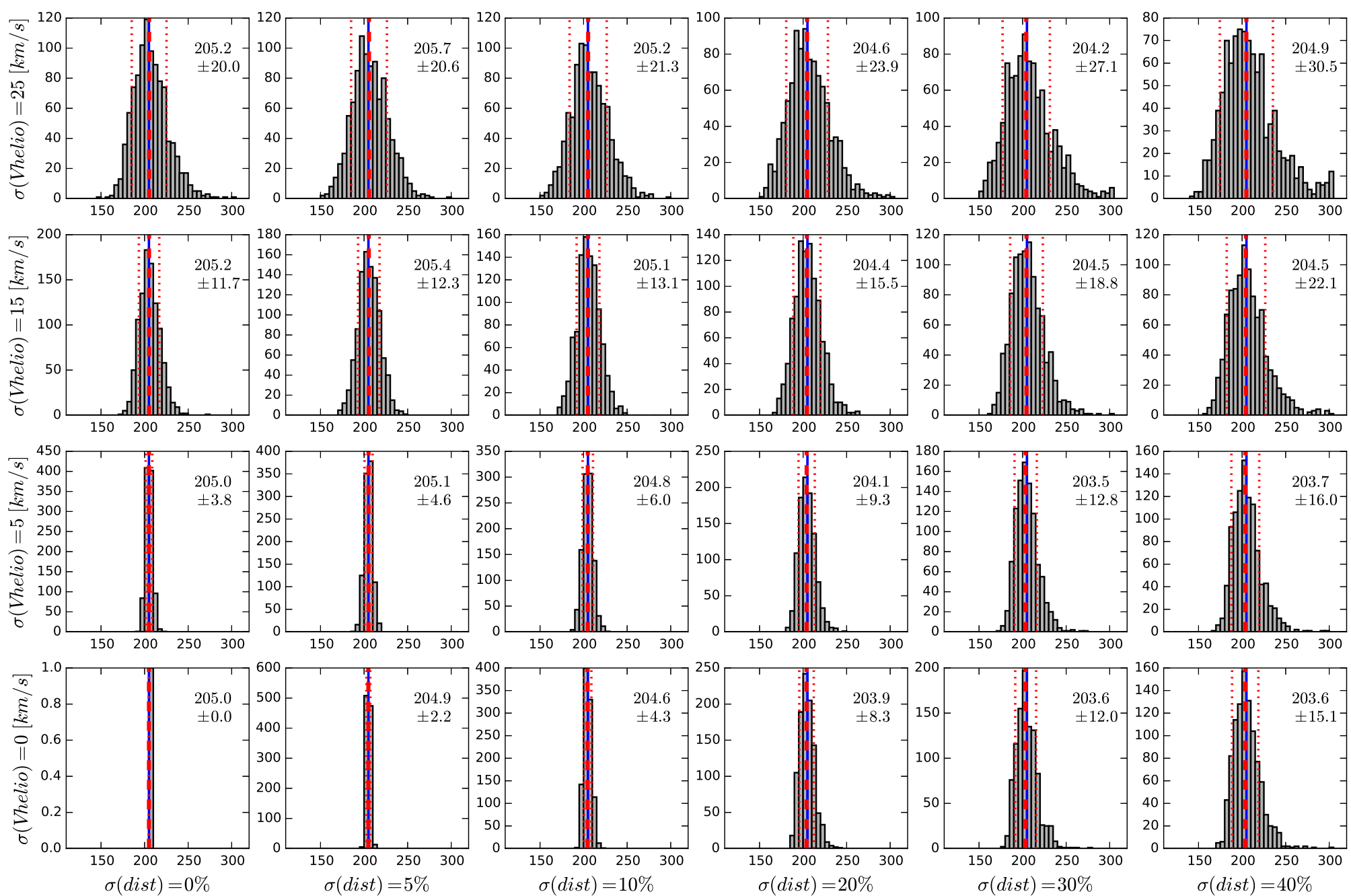

Fig. 4.11. - Same as Figure 4.8, for $\theta_{o}$. Initial value is $\theta_{o}=205 \mathrm{~km} / \mathrm{s}$. 
$V_{c}+V_{\odot}-\theta_{o}[\mathrm{~km} / \mathrm{s}]$
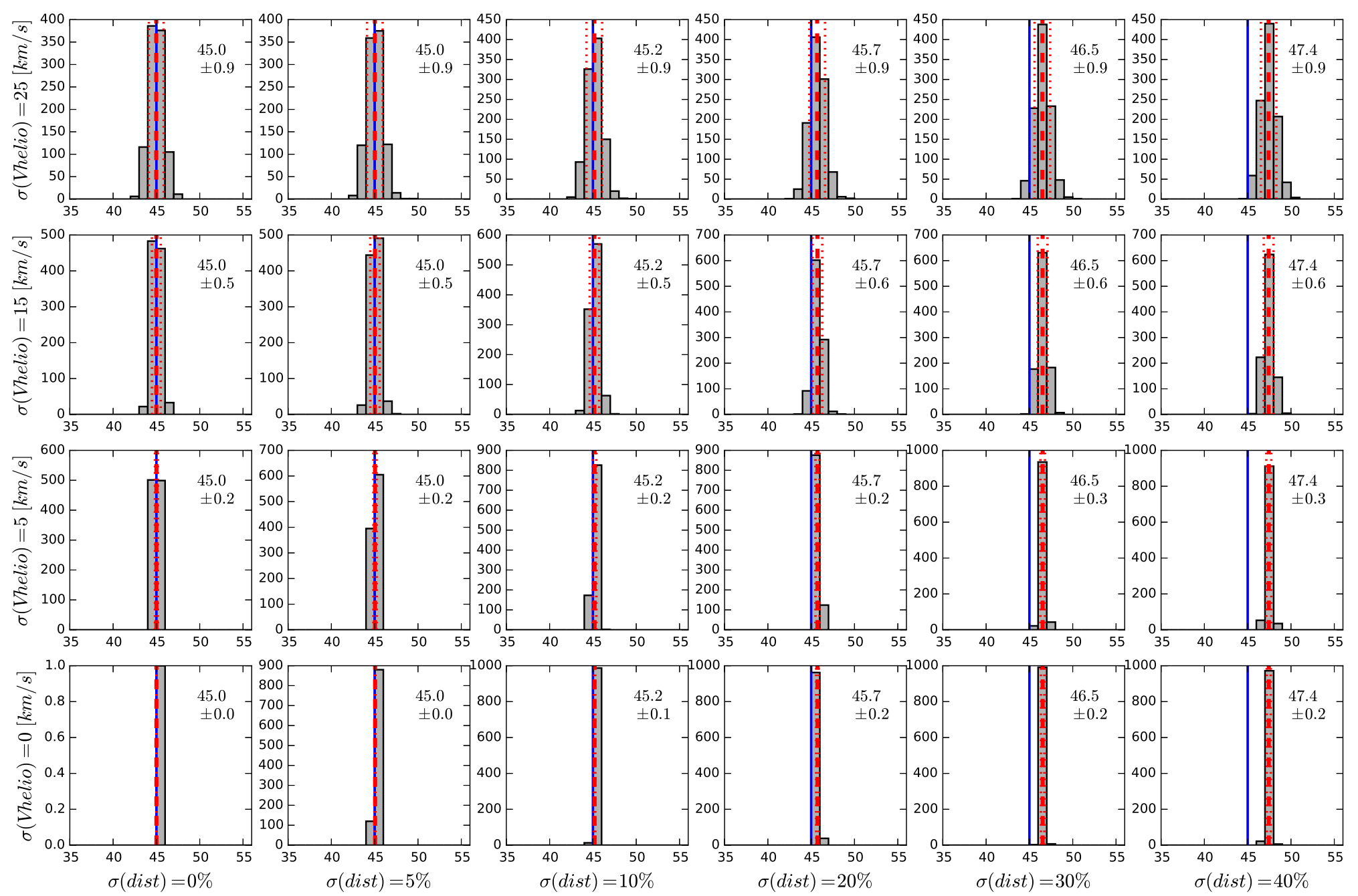

Fig. 4.12.- Same as Figure 4.8, for the difference between the measured total rotation and the rotation of the stellar population. Initial value is $V_{c}+V_{\odot}-\theta_{o}=45 \mathrm{~km} / \mathrm{s}$. 
$\theta_{f}$
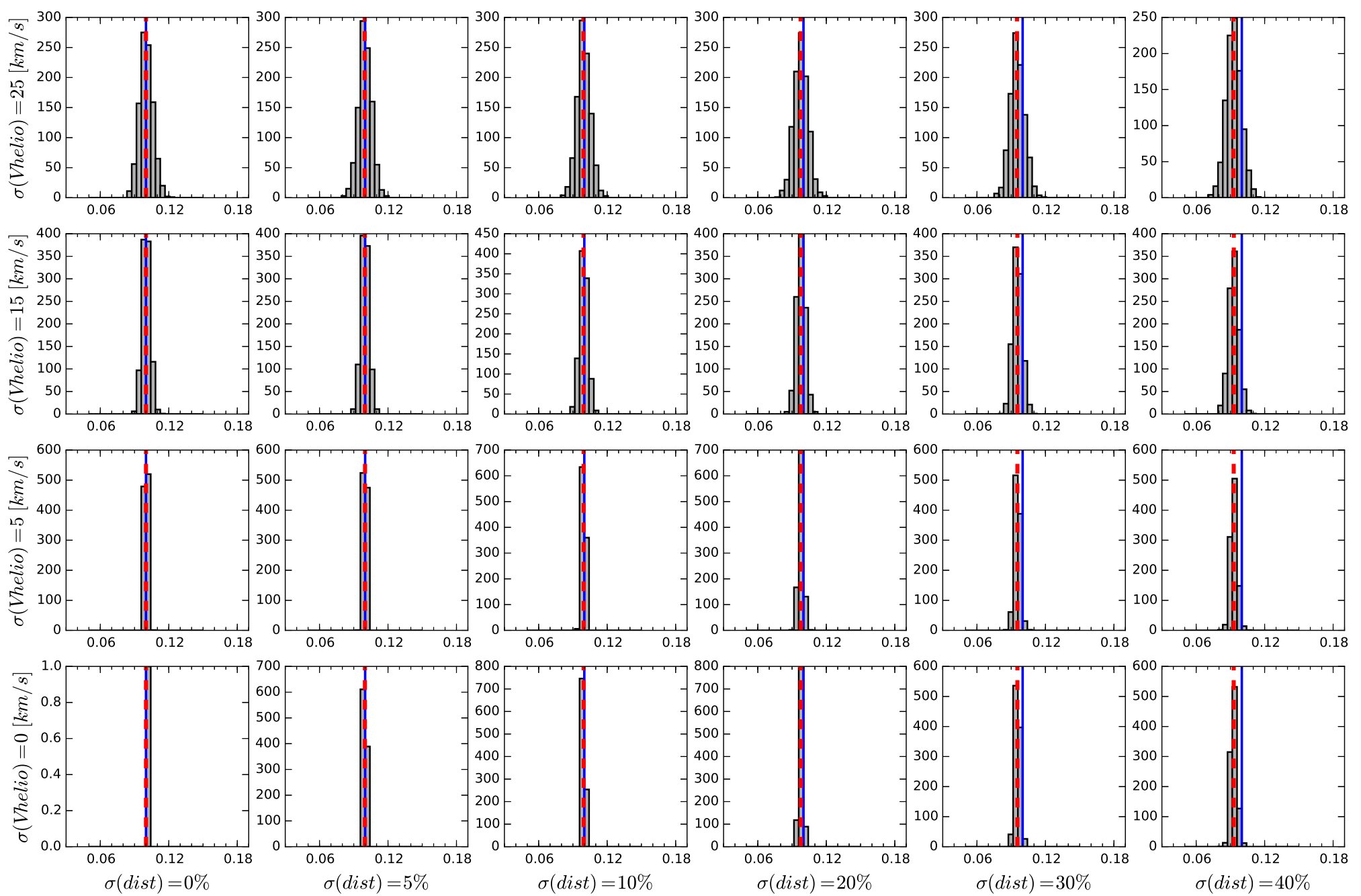

Fig. 4.13.- Same as Figure 4.8, for the velocity drift factor $\theta_{f}$. Initial value is $\theta_{f}=0.1$. 


\section{Chapter 5}

\section{The APOGEE-S Spectrograph: Measurements and Laboratory}

\section{Work}

\section{$5.1 \quad$ Introduction}

This chapter describes contributions I have made to the construction of the APOGEE$2 \mathrm{~S}$ spectrograph, as part of the IR instrumentation team in the UVa Department of Astronomy. The work was directed primarily by Dr. John Wilson, APOGEE Instrument Scientist.

\subsubsection{The APOGEE Surveys in the Context of the Sloan Digital Sky Survey}

The advent of digital technology in the past decades has deeply impacted the way humans pursue knowledge. Astronomy is a clear example of this advancement. The 
development of sensitive digital imaging devices, along with powerful computers, large digital storage units and broadband data networks have opened the door to large systematic surveys covering huge areas of the sky. Within this paradigm, the Sloan Digital Sky Survey (SDSS hereafter) represents one of the most successful observational endeavors in the history of astronomy. SDSS uses a dedicated large field-of-view (3 degree diameter) 2.5-m diameter f/5 telescope (Gunn et al. 2006) located at the Apache Point Observatory (APO), in New Mexico. The telescope can operate in imaging and spectroscopic modes. Observations started over 15 years ago, and the survey has passed through four different phases ${ }^{1}$. Each phase focuses on a set of specific surveys or experiments for a predefined period of time. Each experiment defines a set of clear major science goals and makes use of specific instrumentation, data reduction and calibration pipelines to accomplish them. Telescope time is distributed among experiments during the length of each phase, so that each one fulfills the pre-established observational goals.

The first and second phases, SDSS-I and SDSS-II, occurred between 2001-2005 and 2005-2008, respectively. The final data products of these phases included the "SDSS Legacy Survey" - a photometric catalog in five filters covering 8,240 square degrees plus a $R \sim 2,000$ spectroscopic catalog of over one million of galaxies and 120,000 quasars. SDSS-II also included the "SDSS Supernova Survey" and the "Sloan Extension for Galactic Understanding and Exploration" (SEGUE) survey. The former was a time-domain survey of a region of the sky to detect supernovae and measure their light curves; the latter was a spectroscopic survey of 240,000 thick disk and halo Milky Way stars. These spectroscopic experiments used the original twin SDSS spectrographs, which cover the spectral window from $3,900 \AA$ to $9,100 \AA$, with a resolution $R \sim 2,000$ (Smee et al. 2013). In particular, SEGUE was the first SDSS

\footnotetext{
${ }^{1}$ For detailed descriptions of the experiments, visit http://www.sdss.org
} 
spectroscopic survey specifically devoted to Galactic structure. The SDSS spectrographs delivered an RV accuracy between $4 \mathrm{~km} / \mathrm{s}$ and $15 \mathrm{~km} / \mathrm{s}$, depending on target magnitude (Yanny et al. 2009). In addition, SEGUE estimated stellar metallicities with a precision of $\sim 0.22$ dex.

SDSS-III started data collection in the second half of 2008, and lasted until 2014. This phase executed only spectroscopic surveys: A (one year) extension of SEGUE (SEGUE-2), the "Multi-object APO Radial Velocity Exoplanet Large-area Survey" (MARVELS), the "Baryon Oscillation Spectroscopic Survey" (BOSS), and the "Apache Point Observatory Galactic Evolution Experiment" (APOGEE). SEGUE2 collected spectra for almost 120,000 more stars than SEGUE. It targeted Galactic halo stars ranging from 10 to $60 \mathrm{kpc}$ in distance. All spectroscopic surveys in SDSS-I and -II, plus SEGUE-2, used the two original SDSS optical wavelength spectrographs that could accommodate 640 total optical fibers. The SDSS spectrographs were subsequently upgraded after SEGUE-2 observations concluded to be capable of observing 1,000 fibers simultaneously. Furthermore, the upper wavelength cutoff was increased to $10,000 \AA$ (for a full description of the original and upgraded SDSS spectrographs, see Smee et al. 2013). Using the upgraded spectrographs, BOSS collected spectra of 1.5 million luminous galaxies and 160,000 quasars. Meanwhile the MARVELS experiment built a special optical spectrograph capable of measuring Doppler velocities with $\sim 12 \mathrm{~m} / \mathrm{s}$ precision to search for gaseous giant planets orbiting stars. Complementing these SDSS-III surveys was the APOGEE experiment and its near-infrared spectrograph, described below.

In mid-2014, SDSS entered its current phase — SDSS-IV — , which is expected to be completed in 2020. This phase consists of three experiments, namely, the "Extended Baryon Oscillation Spectroscopic Survey" (eBOSS), the "Mapping Nearby Galaxies 
at APO" (MaNGA) experiment, and the APOGEE-2. The eBOSS survey extends the galaxy sample surveyed by BOSS, both in number and redshift. MaNGA will obtain spatially-resolved optical spectra of 10,000 nearby galaxies, by using 17 fiber bundles —integrated field units - that feed light to the BOSS spectrographs. APOGEE-2 represents a milestone in the history of SDSS, as it is the first SDSS experiment that includes data collected from the Southern Hemisphere. To do so, a second APOGEE spectrograph (almost identical to the original) is being built. This new instrument will be attached to the Irénée du Pont telescope, at Las Campanas Observatory, Chile.

The original APOGEE, carried out during SDSS-III, was a comprehensive highresolution spectroscopic survey of stellar populations in the Milky Way. APOGEE collected over 500,000 spectra of more than 146,000 stars. APOGEE distinguishes itself from other MW surveys by several unique aspects, namely, the gathering of $H$-band infrared, multi-epoch, high-resolution spectroscopy of all stellar populations in the Galaxy (Majewski et al. 2015), even those heavily obscured by dust, given the minimal interstellar extinction at IR wavelengths. The combination of these survey properties results in high-precision radial velocities and chemical abundances $(\sim 100$ $\mathrm{m} / \mathrm{s}$ and $\sim 0.1$ dex, respectively) of stars from the halo, disk and bulge of the Milky Way to large distances.

The success of APOGEE inspired an extension, APOGEE-2, within SDSS-IV. Besides an increase in the number of observed targets, a substantial driver for APOGEE2 is the inclusion of observations from the Southern Hemisphere. The combined observations from APO and Las Campanas will provide a "comprehensive all-sky view of the Milky Way" (Majewski et al. 2015).

Southern Hemisphere observations will be obtained using the 2.5-m, f/7.5 Irénée du Pont telescope — located at Las Campanas observatory, Chile-, through the co- 
operation of the Observatories of the Carnegie Institution for Science, which owns and operates LCO. APOGEE-2 also includes the cooperation of seven Chilean universities, who will provide the local operations manpower for APOGEE-2S. Even though moving the original APOGEE spectrograph to Chile during the second half of SDSS-IV was initially considered to implement the dual hemisphere strategy, additional funding from the Sloan Foundation has enabled the construction of a second spectrograph. The new APOGEE spectrograph is being built with almost identical specifications to its northern sibling, but with several modifications, including earthquake mitigation (Majewski et al. 2015), an improved LN2 tank support, and a simplified VPH grating design. In the following sections, we name the original SDSS-III APOGEE spectrograph as "APOGEE-N" - APOGEE North—, while the instrument being developed for the Southern Hemisphere is "APOGEE-S".

\subsubsection{The APOGEE Spectrographs}

The concept and specifications behind the design of both APOGEE spectrographs are identical, motivated by the primary goal of the APOGEE survey to obtain a homogeneous sample of Milky Way stars from both hemispheres.

A key component of the APOGEE survey strategy is to observe a large number of targets each night. This is achieved with the combination of an efficient telescope (i.e. one with a large étendue), and a spectrograph capable of exploiting the telescope étendue by observing at least a few hundreds of stars in a single telescope pointing.

A frequent implementation for spectroscopic surveys, adopted by APOGEE too, is the use multi-object, fiber-fed spectrographs. Such spectrographs can be attached to the telescope (like the SDSS spectrograph) or, housed in a separate room adjacent to the telescope (like the APOGEE-N, FOBOS, and Hydra spectrographs). The latter 
approach offers the extra advantage of producing very stable instruments, because the gravity vector is constant and independent of telescope orientation. In addition, floor-mounted, fiber-def systems permit the construction of larger and heavier spectrographs.

The APOGEE-N spectrograph is capable of simultaneous observation of up to 300 sources using the same number of optical fibers. These fibers transmit the stellar light from the focal plane of the telescope into the spectrograph. Each fiber needs to be precisely located on the focal plane to match the position of an astronomical target. For this, one end of each fiber is inserted into a hole precisely-drilled for each target on an aluminum plate. Each plate is drilled specifically for a single location on the sky, based on astrometric catalogs. During observing, up to nine pre-plugged plates can be mounted at the focal plane of the telescope. The other end of the fibers is located in the interior of the spectrograph, whose body keeps a cryogenic and evacuated environment. Cryogenic temperatures are reached in the evacuated environment using LN2, and the implementation — within the cryostat - of a radiation shield that is surrounded by multilayer insulation blankets. These blankets have the purpose of shielding the interior of the instrument from the thermal radiation (in IR wavelengths) that comes from the exterior at room temperature, which is the major contributor of stray heat into the instrument. Inside the cryostat (Figure 5.1), the optical components lie on an aluminum cold plate that serves as an optical bench. The fibers end in an approximately vertical array forming a spectrograph pseudoslit. Then, light exiting the fibers reaches a collimator, a Volume Phase Holographic (VPH) transmissive dispersion grating, and an f/1.4 imaging camera, before reaching an array of detectors in a 3x1 configuration. This configuration results in a nominal spectral resolution of $R \sim 22,500$. 


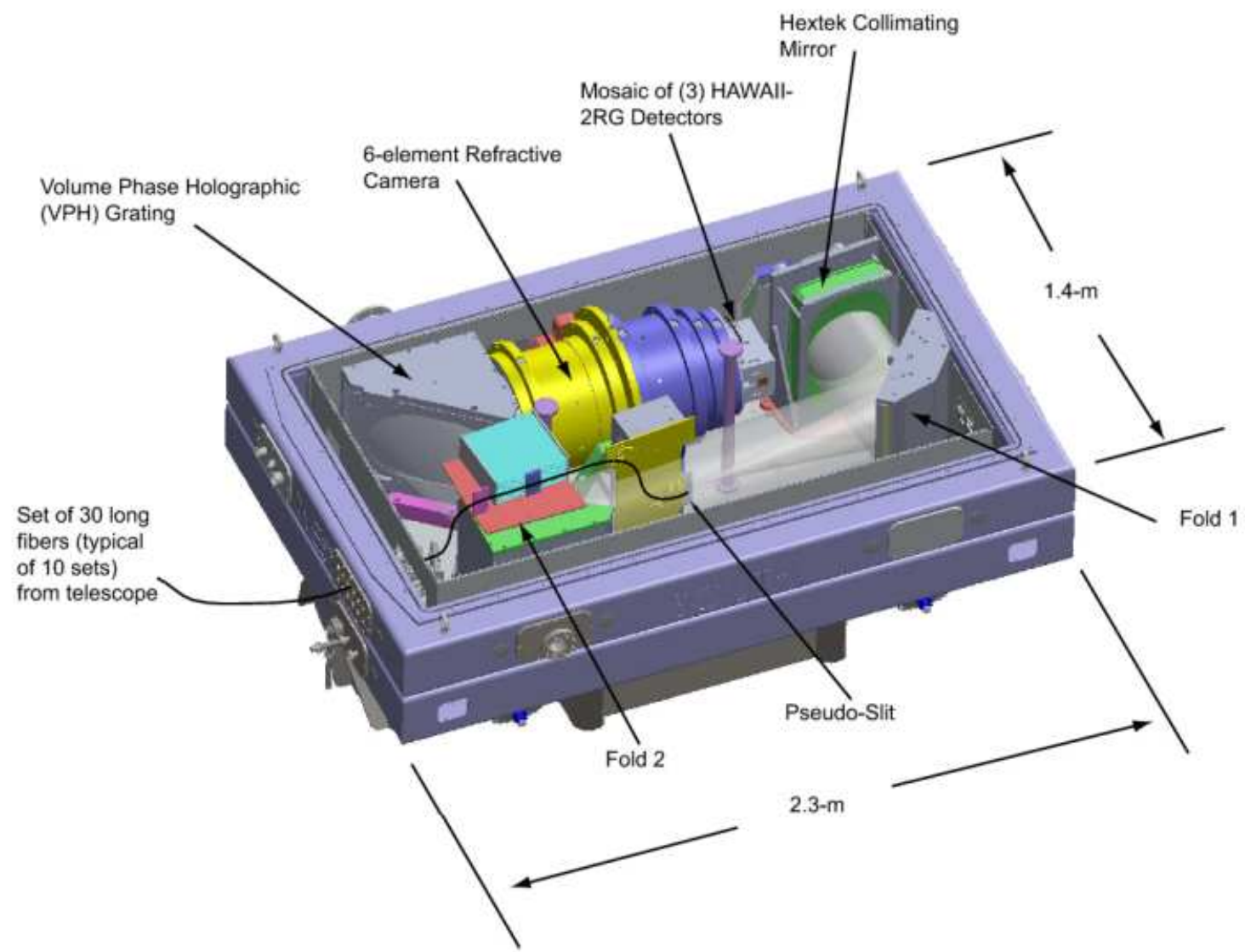

Fig. 5.1. - Layout of the APOGEE spectrograph within the optical bench. Figure from Majewski et al. (2015)

The APOGEE-S spectrograph is currently being integrated in the Virginia Astronomical Instrumentation Laboratory (VAIL), under the directorship of lab director Michael Skrutskie and APOGEE Instrument Scientist John Wilson, at the Astronomy Department of the University of Virginia, with nearly identical specifications as the APOGEE-N instrument. The new spectrograph is scheduled to be shipped to Chile during mid-2016.

The following sections describe three tasks carried out as part of the construction of the APOGEE-S spectrograph, in which I player a leading role. 


\subsection{Testing the VPH Grating for the APOGEE-S Spectrograph}

\subsubsection{Motivation}

The APOGEE spectrographs use a Volume Phase Holographic grating (hereafter $\mathrm{VPH})$ as its dispersive element. A VPH is a diffractive optical element fabricated using an advanced technique that imprints an interference pattern, created with a laser, onto a gelatin substrate. The interference fringes create a hologram that change the refractive index in the gelatin, producing a periodic structure that modulates the phase of light that passes through it. The result is a grating that is usually used in transmissive mode and whose peak diffraction efficiency is very high. Details on the optical design and construction of the APOGEE-N VPH are described in detail by Arns et al. (2010).

The APOGEE spectrographs require large VPH elements $(290 \mathrm{~mm} \times 475 \mathrm{~mm})$. The principal challenge for the construction of this disperser is the size, because the image that VPH manufacturers record the hologram on the substrate is not as large as the required VPH. To overcome this, the APOGEE-N VPH substrate was exposed as a precisely positioned mosaic of three separate holograms. In the case of APOGEE$\mathrm{S}$, it was determined that almost similar performance was attainable with a simpler two-image mosaic (figure 5.2). Precise translation of the VPH substrate is required between exposures. Any differences in line density between the two panels will produce a misalignment in the output beams passing through each side of the VPH. This will result in degraded image quality of the spectral features in the spectrograph.

In the following sections, we describe a method to measure and quantify any misalignment between the beams from the independent VPH panels for the APOGEE- 


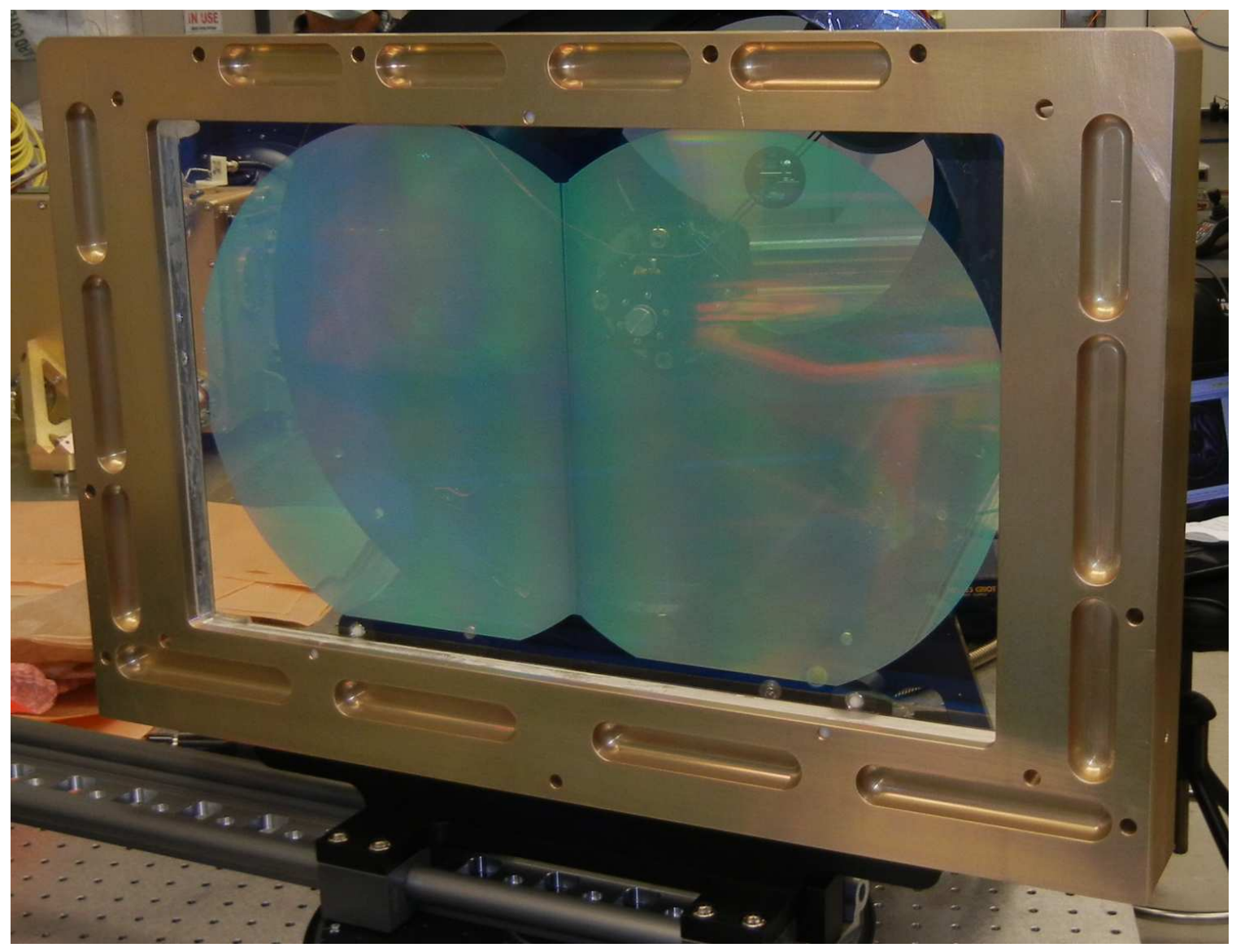

Fig. 5.2.- An image of the APOGEE-S VPH. The recorded holograms of the left and right panels of the $\mathrm{VPH}$ are visible.

S VPH candidate. These results have been used as an independent verification of two other tests made by other researchers using a laser interferometer in the output beams that pass through both VPH panels.

\subsubsection{Data Acquisition and Description}

On November 7th, 2014, Dr. John Wilson (University of Virginia) and Dr. Robert Barkhouser (Johns Hopkins University) collected several datasets in the facilities of the Instrument Development Group at Johns Hopkins University. These datasets were used to assess the VPH quality using three different methods. The first and 
second methods are based on interferometric fringes, using a Laser Unequal Path Interferometer (hereafter LUPI). The third method, which we present here, images the spots produced by the LUPI after passing through the left and right panels of the VPH, and determines the shift in the position of the spots on the focal plane of an imaging device. To obtain meaningful results with this method, we must obtain three subsets of images taken with different optical configurations. We describe these configurations below.

The first configuration (Figure 5.3) is designed to measure the VPH quality. First, the LUPI — equipped with a HeNe laser emitting at a wavelength of $632.8 \mathrm{~nm}$ - with an $\mathrm{f} / 10$ diverger is used as a light source. This beam is collimated by positioning the LUPI at the focal plane of a 15" f/12 Ritchey-Chrétien system. Next, the collimated beam passes through the VPH with an angle of incidence of $\theta_{i}=18.61$ degrees. This angle satisfies the Littrow condition $2 d \sin \theta=m \lambda$, at order $m=1$ for the laser wavelength and VPH groove density. This angle condition corresponds to the actual setup of the VPH in the spectrograph, although the angle for Littrow condition for NIR operation is $54^{\circ}$, for $\lambda=1.604195 \mu \mathrm{m}$. The transmitted beam is imaged by a Meade LX200 ACF 12" f/10 Schmidt-Cassegrain system. This telescope has a Nikon D5000 digital camera attached to its Cassegrain focus using a T-ring. This camera has a sensor of $4310 \times 2868$ pixels and a physical size of $(23.6 \times 15.8) \mathrm{mm}$. All images from the camera were saved in Nikon's lossless NEF image format. In addition, the imaging system is equipped with a graduated fine-focus knob. Furthermore, the researchers can cover each half of the $\mathrm{VPH}$, which is useful to image the laser spot passing through each individual VPH panel.

Wilson and Barkhouser took three different series of images with this configuration. In all cases, the exposure times and ISO sensitivity of the camera were chosen 


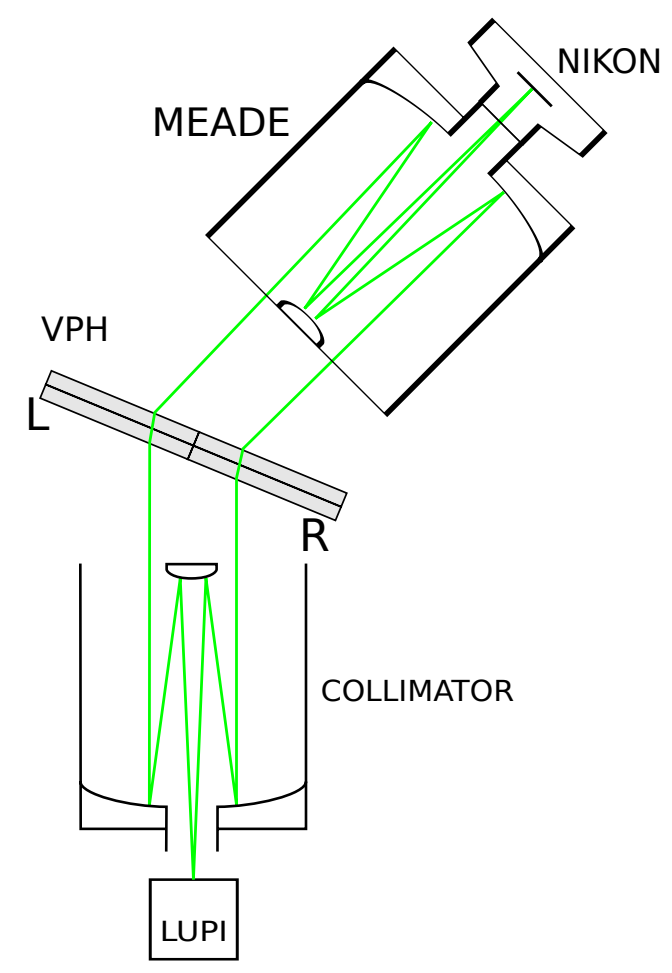

Fig. 5.3.- Optical configuration used to collect the data used to measure the VPH image quality.

to reach a high signal-to-noise, while preventing saturation. The first sequence has both VPH panels open. The second and third sets have the left and right panel covered, respectively. Each of these three sequences contains 43 images, with focus values ranging between 3 and 12 units. The focus knob is adjusted by 0.25 units between frames. Exposure times were adjusted accordingly as focus changed, and range between $1 " / 640$ and $1 " / 1250$.

The second optical configuration is shown in Figure 5.4. This setup has the VPH removed from the optical path and is intended to measure the root mean square (RMS) radius of the collimated laser beam at best focus. The focus values of this sequence ranges between -2 and 2 , with increments of 0.25 units. The zero-point of the focus scale is set at the best focus. 


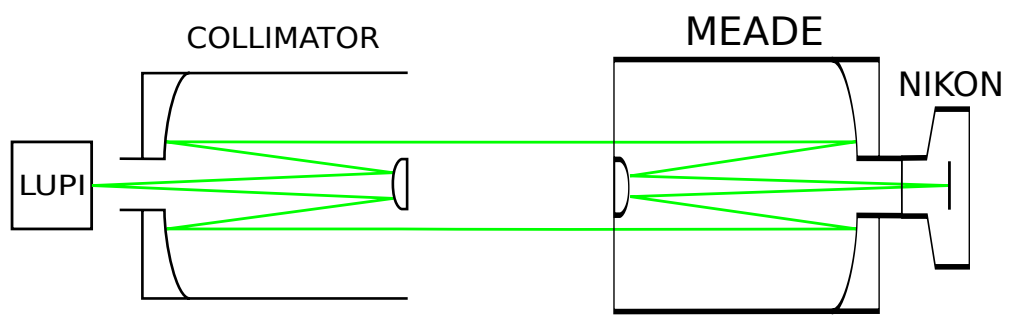

Fig. 5.4.- Optical configuration to measure the natural spot size of the laser from the LUPI, after passing through the dispersor, collimator and imaging systems.

The third optical configuration is designed to derive the plate scale of the imaging system, which is required to transform our measurements from pixel units into angular units. In this setup, shown in Figure 5.5, the Meade telescope is illuminated with the image of the cross-hairs from a theodolite pointed at different angles of incidence. Changes in this angle translates into a displacement of the image of the cross-hairs on the focal plane imaged by the Nikon. The theodolite reports the cross-hair azimuth and altitude of the angular displacement of the input beam. The dataset for plate scale measurements contains seven images, one for each angular position. The different positions of the cross-hairs in the sequence are shown in (figure 5.6).

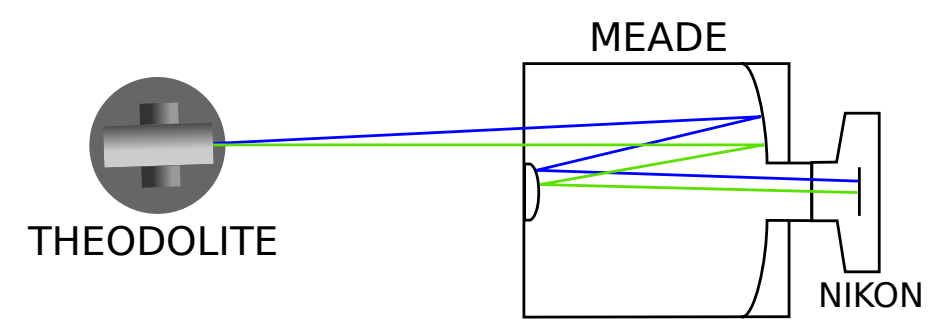

Fig. 5.5.- Optical setup to estimate the plate scale with a theodolite placed in front of the imaging equipment. Changes in the angle of incidence of the beam, modified by moving the theodolite, correspond to translation of the cross-hair image on the focal plane of the Meade+Nikon system. Incidence angles are read from the theodolite. 


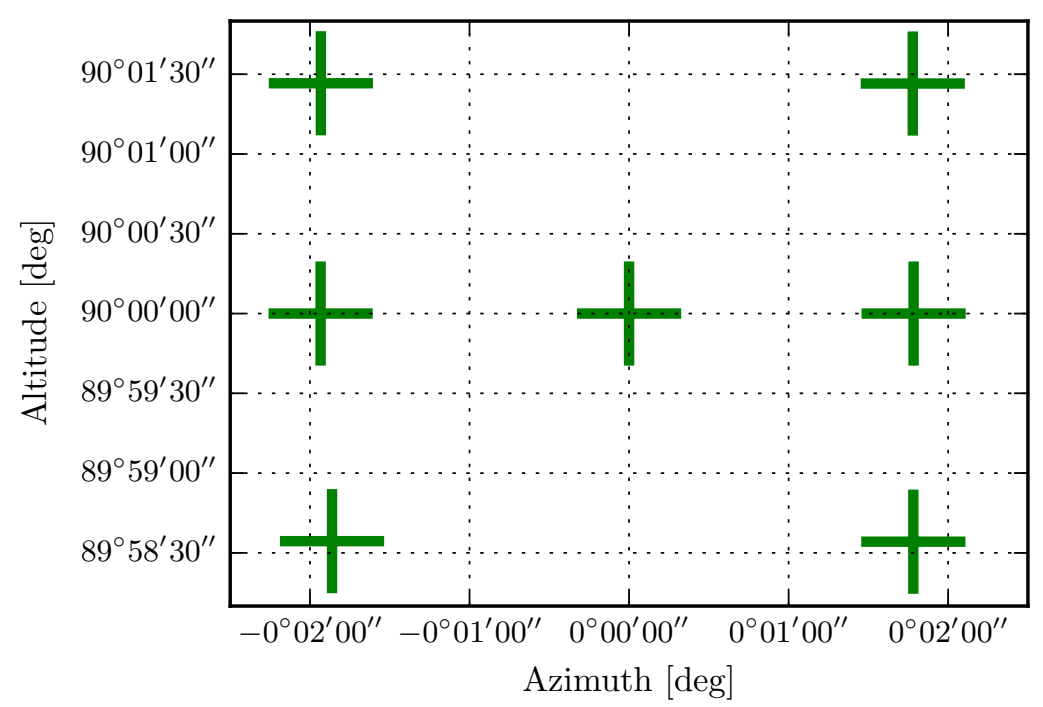

Fig. 5.6. - Angular positions of the theodolite cross-hairs, read from the theodolite, that are imaged with the MEADE+Nikon system.

\subsubsection{Measurement Method and Results}

In this section, we describe the rationale and methods employed to derive meaningful quantities from the datasets described above.

First, we present the equations for two quantities that are commonly used in optical applications to characterize imaged light spots in terms of position and size. These quantities are the centroid or center of mass (CM, hereafter), and the Root Mean Square radius (RMS radius, hereafter), respectively. The equations shown below are adapted from the expressions used in ray tracing applications. In our case - for data collected as digital images - we assume that the response from the detector is linear and that each digital count represents a photon arriving at the detector from the optical system. In this way, each count corresponds to one ray under the ray-tracing approach.

For images with Cartesian $(x, y)$ coordinates, the coordinates of the CM $\left(X_{C M}, Y_{C M}\right)$ are defined as: 


$$
X_{C M}=\frac{\sum_{i=0}^{N} I_{i} x_{i}}{\sum_{i=0}^{N} I_{i}}, \quad Y_{C M}=\frac{\sum_{i=0}^{N} I_{i} y_{i}}{\sum_{i=0}^{N} I_{i}},
$$

where $\left(x_{i}, y_{i}\right)$ and $I_{i}$ are the coordinates and number of counts of the $\mathrm{i}$-th pixel, respectively; and the summation is over the $\mathrm{N}$ pixels of the image.

Similarly, the root mean square radius (RMS radius) is:

$$
R M S=\sqrt{\frac{\sum_{i=1}^{N} I_{i}\left[\left(X_{i}-X_{C M}\right)^{2}+\left(Y_{i}-Y_{C M}\right)^{2}\right]}{\sum_{i=1}^{N} I_{i}}}
$$

We measure these parameters for the laser spots imaged in the three optical configurations described previously. The aim is to trace the CM and RMS radius for the spots produced by the left and right VPH panels, for the image sequences along all focus values. The RMS radii are used to find the image with best focus in each sequence, and the CMs are used for a direct measure of the distance on the focal plane between the positions of the spots.

We started with a visual inspection of the three sequences - left-only, right-only, and both-panels - and determined that the features imaged through the left and right VPH panels can be traced, at least qualitatively, in the frames that image both panels simultaneously. We also determined that the spots formed by left and right panels illuminated simultaneously overlap in images that are at or close to best focus. Therefore, data from single panels become important because they allow us to identify the contribution from each panel to the features using both sides. Figure (5.7) shows some examples images of the spots from the left, both and right panels of the VPH. From the figure, it is clear that the data from both panels correspond to the addition of features from left and right sides. However, further inspection of the whole dataset reveals that the focus position for the three sets is not precisely the same for the recorded positions, based on the change of the shape of the features. This introduced 
some problems that we will explain and solve below.

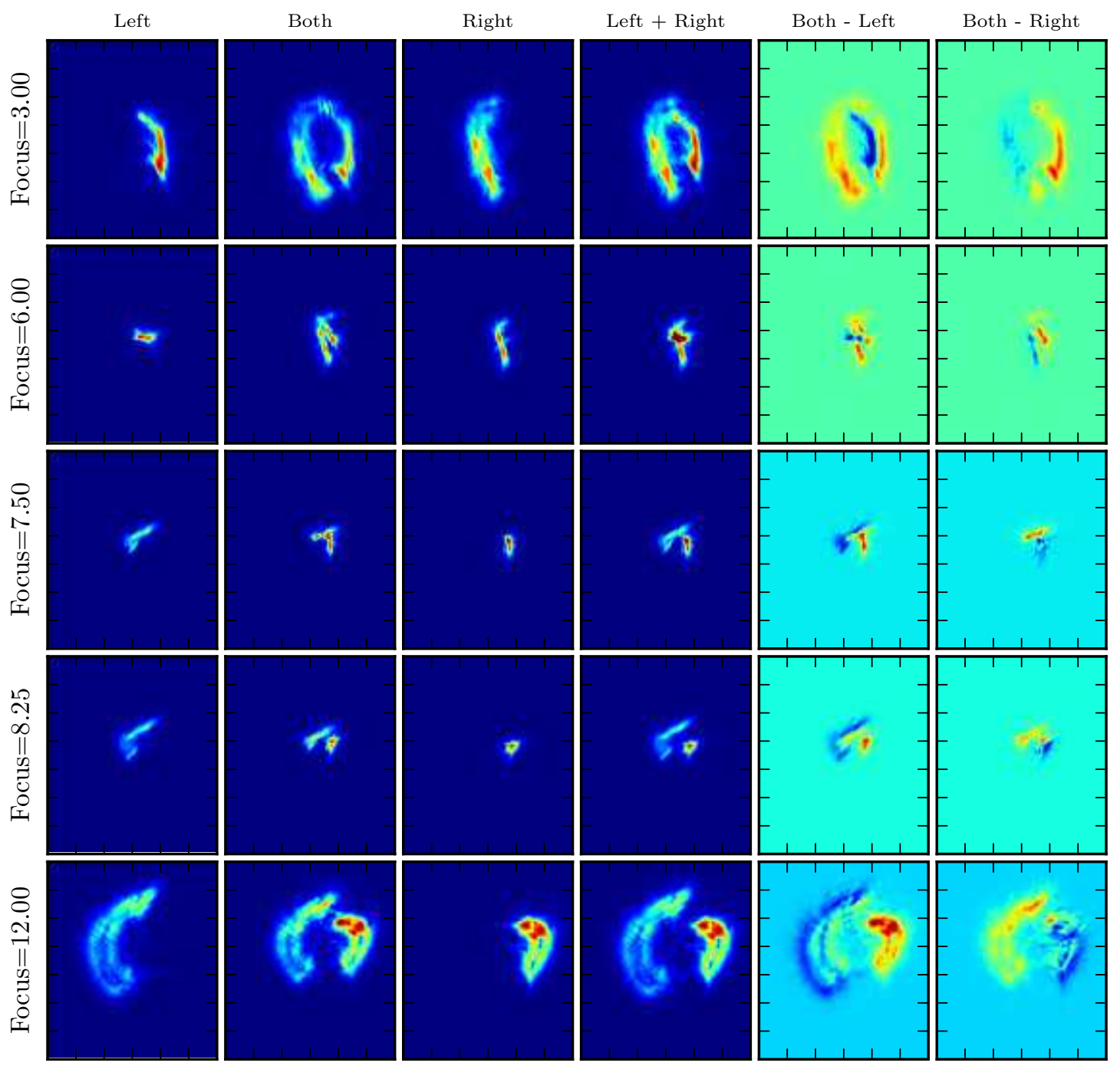

Fig. 5.7. - Example images for the three VPH sequences - left-, both- and rightpanels - at five different focus values. These values correspond to the farthest in focus and out of focus positions in the sets (3.0 and 12.0); and the best focus for the left-, both-, and right-panel sequences (6.00, 7.50 and 8.25 units, respectively). The plots on left + right, both - left and both - right columns represent the image arithmetic made with the three datasets, although no registration has been applied to these images.

Next, we determine the best focus position for the three sequences by measuring 
the RMS radii, which occurs at the minimum RMS radius value. In the analysis, we trimmed the images to a rectangular area of $740 \times 720$ pixels. Features in all images are relatively small and well contained inside this area. The panels on the left column of Figure 5.8 show the RMS radii for the sequences. The measurements follow a rather well-behaved curve, described by a second order polynomial fitted to the RMS radius squared. However, there is noise in the measurements, as can be seen for some RMS radius values that deviate from the smooth curve described by the fit. The worst case happens for the measurements of the right VPH panel, where the minimum RMS from the fit and from the images have best focus values that differ by almost two units. We checked the data for this image in more detail and determined that a few noisy pixels at large radii from the CM can increase the RMS by a large amount, due to the quadratic distance terms in Equation 5.2.

To solve the problem of noisy pixels, we developed some computer routines that mask out such pixels in the images. To do so, we create a mask defined by the contour of the isophote whose intensity is higher than some defined threshold. Then, we set pixels outside of this contour to zero.

In practice, we trace isophotes using the contour function from the Matplotlib plotting library in Python. After some minor hacking of the function, we can obtain the actual image coordinates of the vertices of the polygon that trace an arbitrary isophote set by us. We set the mask boundary at three counts. The average background level is $\sim 0.1$ counts, and its standard deviation is around $0.2-0.3$ counts. However, the data are stored as integer values. Therefore, we choose 3 counts for the isophote level, because 1 or 2 counts did not guarantee no effect by noisy pixels. Furthermore, the highest intensities in a spot usually reach over 100 counts. Figure 5.9 shows an example of the spot selection using contours. Through this method, we 


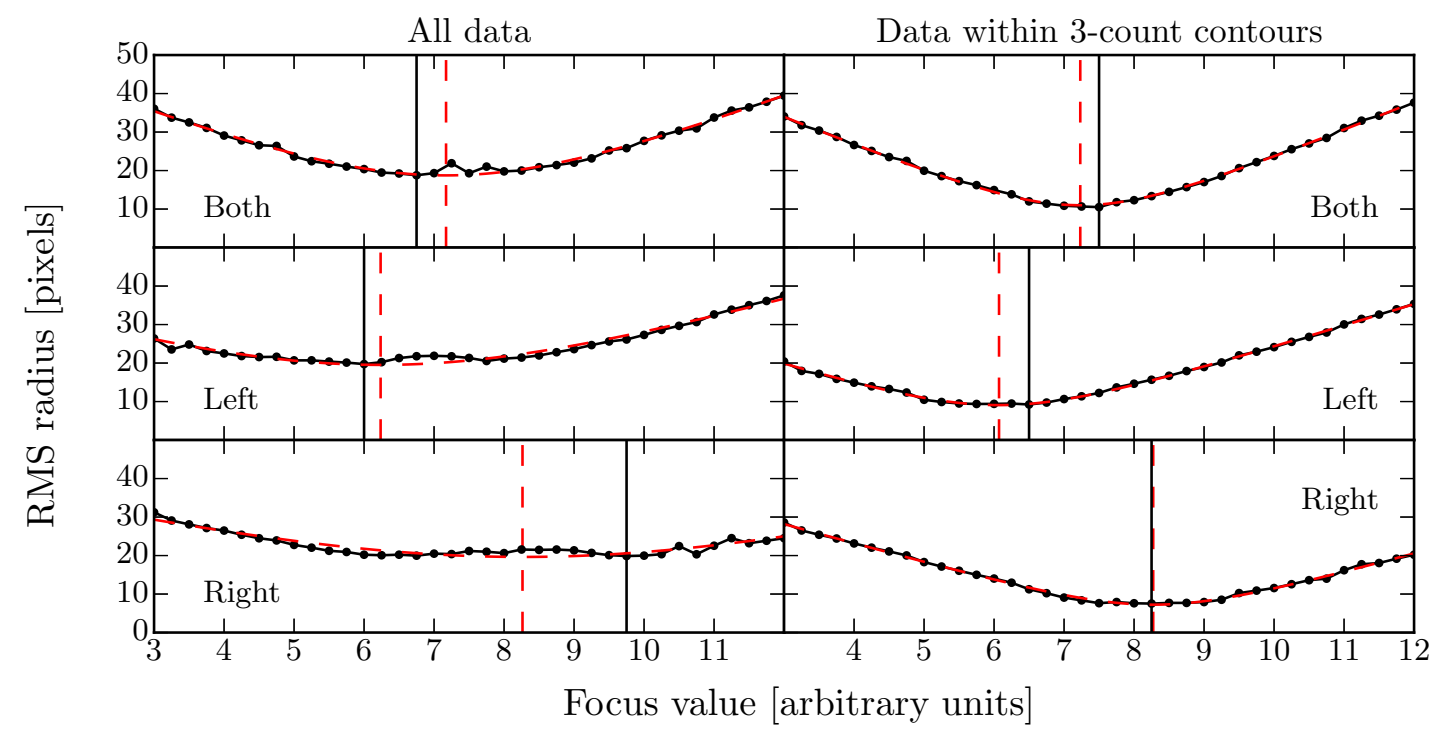

Fig. 5.8. - Results for RMS radii for the three image sequences (both, left and right VPH panels). The focus ranges between 3 and 12 units. Panels on the left include data from the entire image section used in the analysis, while panels on the right only include pixels within a contour that encloses the laser spot and that satisfies $I_{i}>3$ counts. The vertical black line marks the smallest RMS radius of the sequence. The red segmented line is a fit to the data, and the vertical red line is the minimum RMS radius from the fit. The best focus value in each sequence corresponds to the lowest RMS radius.

obtained the RMS radii measurements displayed in the right column of Figure 5.8. It is clear that this method reduces the noise in the rms radii estimations, and leads to a better agreement between the best focus measurements using the quadratic fit and the minimum value measured from images directly.

The next step is to measure the divergence of the laser spots formed by each VPH panel. For this, we calculate the distance between the CM of the spots from both panels on the focal plane of the imaging system. Furthermore, we want to measure the distance as a function of focus position.

If we observe the features in the Both column of Figure 5.7, and compare them to the Left and Right columns, we can see that the spots from left and right panels do 

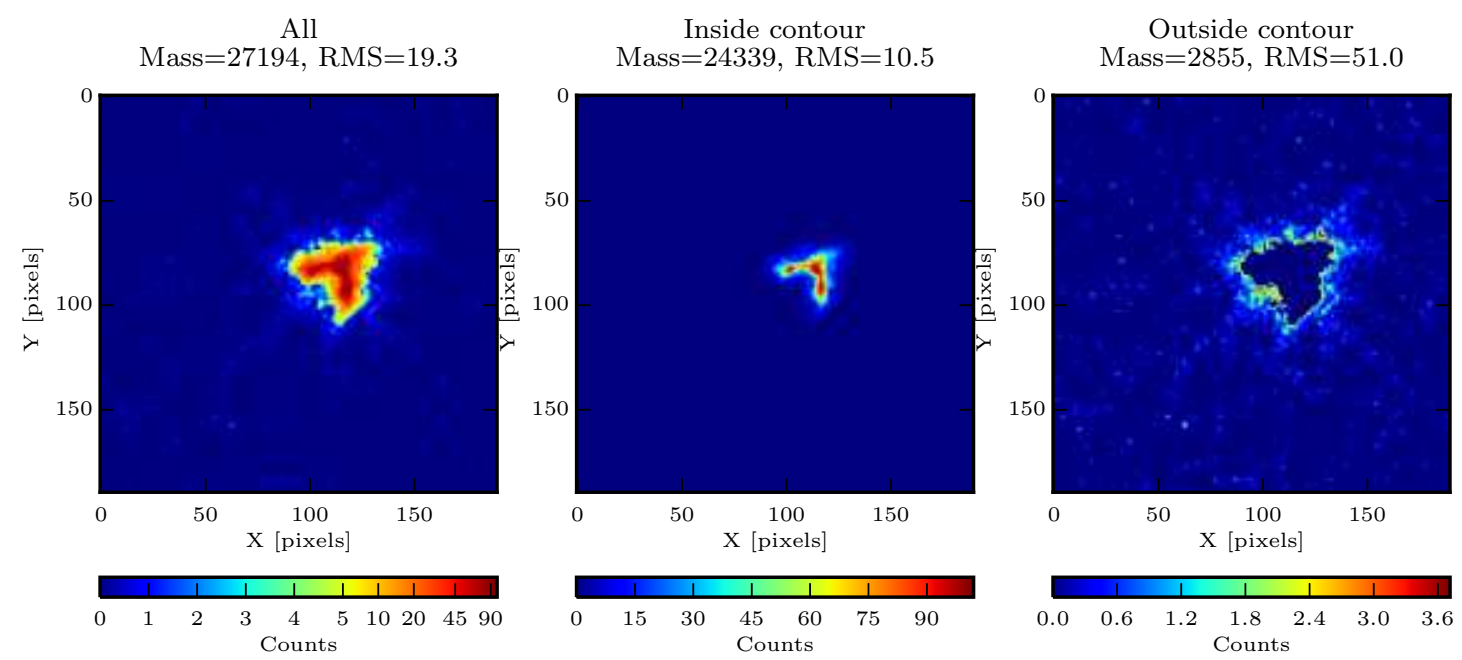

Fig. 5.9. - The contour method applied to the best focus image in the sequence with both VPH panels open. Left: results obtained if we include all pixels. Middle: results using only data within the contour defined by the isophote with intensity of three counts. Right: same measurements, for pixels that fail the criteria for the middle panel (i.e. they lie outside the 3-count isophote of the main spot). The title of each panel shows the RMS radius (in pixels) and total counts (mass, in counts) measured in each case. The color-coding represents pixel intensity. Note that the scales are different, and they are optimized to span the dynamic range of the data in each panel.

not overlap for images with focus values at the edges of the focus sequence. However, the spots do overlap for images close to the best focus values.

Ideally, we could measure the CM for the spots using the left- and right-panel sequences, and calculate the CM separation between them. However, we found two problems in the dataset that make this approach unfeasible, unless we correct for them. First, the focus position for the three sequences do not coincide. Even though the sequences have the same reads from the fine-focus knob, it is clear that the size and shape features imaged using a single VPH panel do not coincide, as it was expected, with the features imaged using both VPH panels at the same focus value (compare "Both" to "left+right" in Figure 5.7). Second, the relative position of the imaging 
system, with respect to the incoming rays, is different for the three image sequences. This translates into a relative shift of the spots imaged in the focal plane among the three sequences, which must be corrected before we can measure separations between the left- and right-panel sequences. As a result, we need to employ two methods to measure the CM. We choose one over the other based on whether the spots overlap or not.

First, we describe the case when spots from the two VPH halves do not overlap (see first and last row in 5.7). In this case, both spots can be traced and isolated following a procedure similar to the image masking method already discussed to measure the CM. We refer to this method as the contour splitting method. We wrote a computer routine that uses contours to find two features, based on Matplotlib's contour function. We expect that contours will trace the isophotes of the two separate laser spots, and that they will form two closed polygons. To implement this, we define two isophotal levels that are important to the process. On one side, a low-intensity isophote usually set at two counts. This isophote defines a contour that encloses the whole feature produced by both VPH panels together, but rejects the noisy pixels from the background. As we already have shown, such noisy pixels hinder the RMS measurements. On the other hand, we define a high-intensity isophote, usually at around 20-25 counts, that splits the large spot and produces two separate features - the two laser spots that we want to measure - and that are contained within the contour defined at 2 counts. The intensity of this contour varies from image to image. Initially, we tried to find this value in an automated way. However, results were not consistent and finding the correct values by-hand worked well, provided that we only have 26 images to inspect. Finally, we use the two high-intensity contours that enclose the two laser spots to apply the masking method (described above) to measure the CM of each spot. 

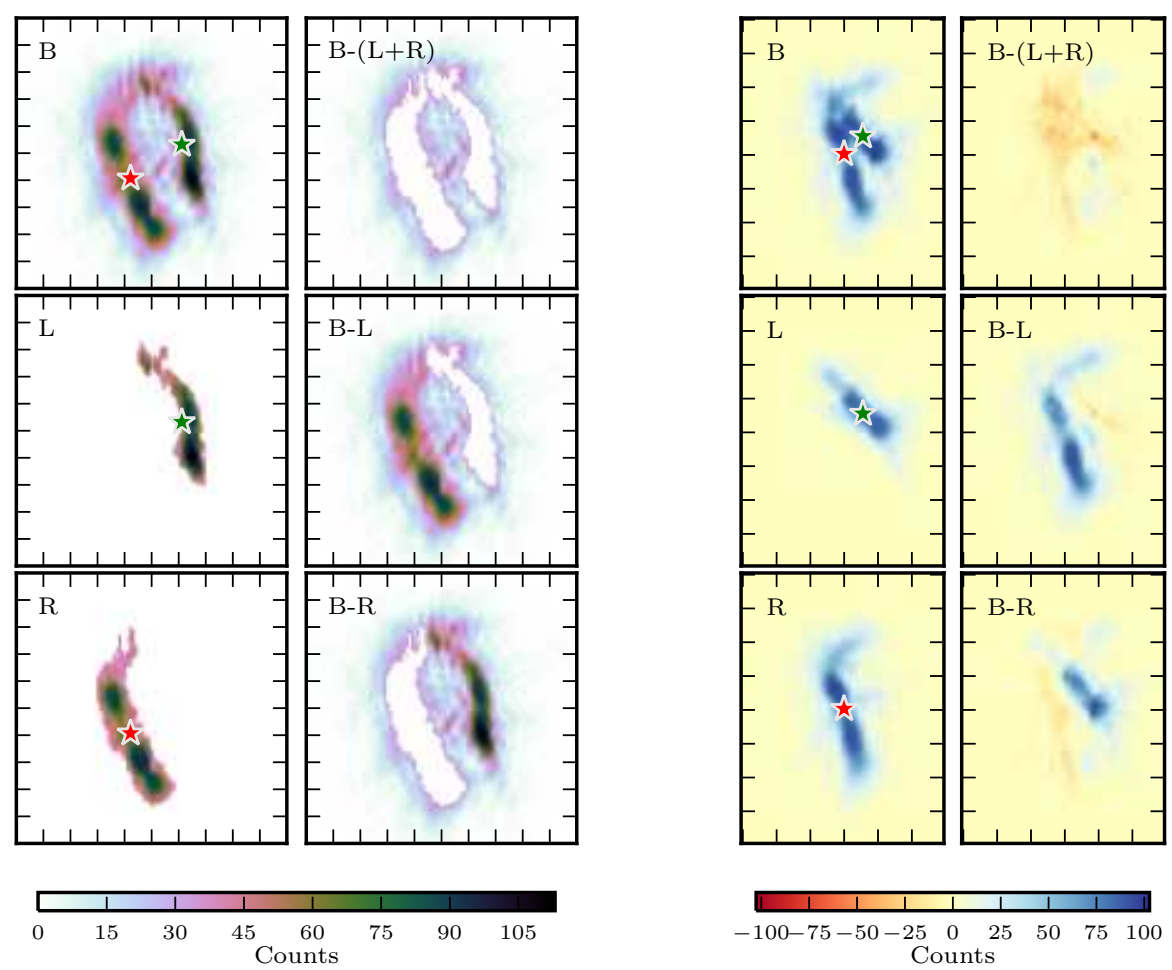

Fig. 5.10.- Center of mass (CM) and RMS measurement methods. The six panels on the left side show the contour splitting method, meanwhile the six panels on the right illustrate the shifting and matching method for features using left- or right-panel images. In both cases, B represents the section of interest for the image from both VPH panels. In the case of contour splitting, $L$ and $R$ are the image sections selected by the contour as being originated by the left or right VPH halves, respectively; while in the other case, they represent the best feature-matching images from the left or right datasets, respectively. The other panels show the results of subtracting the corresponding $\mathrm{L}$ or $\mathrm{R}$ images. The red and green stars mark the position measured for the $\mathrm{CM}$ of the spot created by the left and right panel of the VPH, respectively.

Next, we describe the case where spots from the two VPH halves overlap. We cannot measure CMs of overlapping features directly from images in the "both-panels" sequence because we do not know how many counts each spot contributes to each pixel. Thus, measuring the CMs is more difficult than in the previous case. Nevertheless, we developed a second method that gives satisfactory results. We call this approach the shifting and matching method. The idea is simple. We look for a pair 
of images from the left- and right-panel sequences, whose spots when added together result in the spot produced by the sequence using both VPH panels. Even though we see similar features, both in size and orientation, between the images from left and both — or right and both- sequences (see Figure 5.7), the best matching image from each set will usually have a different focus value. This mismatch in focus scales is also seen in the best-focus measurements in Figure 5.8. However, we could not determine whether the disagreement is only due to offsets in the focus scale, or if a real difference exists between images from each panel. Keeping this in mind, we found the best-matching features among the images in the sequences, and manually shifted the location and adjusted the intensity of the spot from the left- and right-panel sequences, until they produce the best feature subtraction in the image using both VPH panels. We show an example in the right side of Figure 5.10. We usually can discriminate a shift uncertainty of two pixels in each axis, although the subtraction is not perfect. One reason for this uneven subtraction is that the images in each sequence were collected every 0.25 focus step, but sampled at different points. As a result, the features in the three sequences look quite similar, but they do not match precisely. We would expect a smaller uncertainty and a better feature subtraction from this method if images for the three sequences were collected one right after the other. This would ensure that they share the same focus value. Finally, we measured the $\mathrm{CM}$ for each image from left- and right-panel sequences that match the image using both panels, and added the respective position offset from the estimated shift to the measured $\left(X_{C M}, Y_{C M}\right)$ coordinates. Again, we used the masking technique to suppress background noise. We repeat this process for all images with overlapping spots in the both-panel sequence.

With the results from these two methods, we can calculate the distance between 
the CM for the laser spot produced by the left- and right-panel of the VPH for the whole focus range spanning the dataset. However, these results are still in pixels units. Therefore, we need to calculate the plate scale of the system to convert our measurements into angular separations.

Measuring the plate scale of the imaging system is simple. Basically, we need to obtain the distance at the focal plane, in pixel units, between pairs of points with known angular separation. The more pairs we measure, the better precision we achieve in the derived plate scale. For this, we use the dataset of the theodolite cross-hairs imaged with the MEADE+Nikon. First, we measure the $(x, y)$ position (in pixel coordinates) of the cross-hairs in each frame, by means of 2-D cross-correlation. The template (Figure 5.11) is a subsection of one of the images, and encloses the central region of the cross-hairs. We use the $2 \mathrm{~d}$ cross-correlation function from the Scipy package in Python. The results give the image coordinates of the cross-hairs for the seven images in the dataset, with a 1-pixel precision. Then, we calculated the Euclidean distance between cross-hairs for each pair of images, which result in 21 total pairs.

Next, we obtained the angular separation between pairs of cross-hair AltAz positions — read from the theodolite - using the angular distance as defined in spherical trigonometry. The AltAz positions reported by the theodolite are shown in Figure 5.6. Again, we calculated angular separation for the 21 possible pairs. Finally, the plate scale of the system is simply the ratio between angular separation and pixel distance. We calculate the average and standard deviation of the 21 measurements, and obtain $0.351 \pm 0.003 \mathrm{arcsec} /$ pixel as the plate scale and its uncertainty. We present a histogram with the results in Figure 5.12. Given the precision that we can achieve, we rounded the plate scale value to $0.35 \mathrm{arcsec} / \mathrm{pixel}$. 

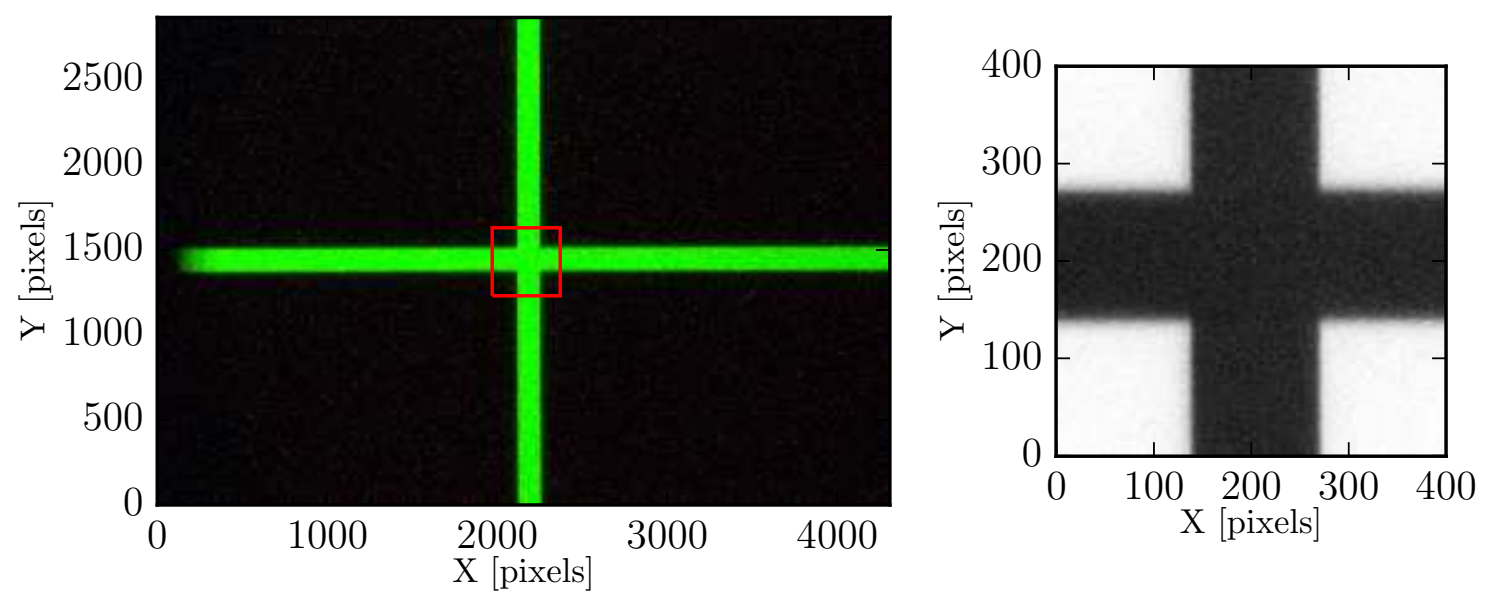

Fig. 5.11.- Left: the theodolite cross-hairs, as imaged by the Meade+Nikon setup, in RGB color space. The red box marks the image section used as cross-correlation template. Right: the cross-correlation template converted to gray scale for crosscorrelation.

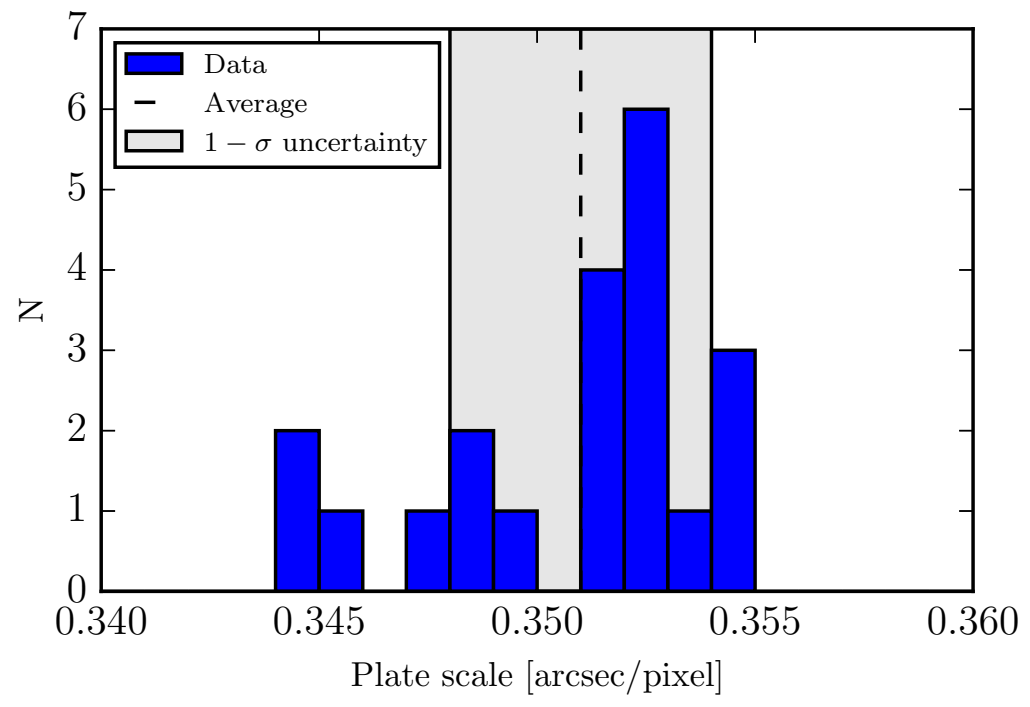

Fig. 5.12. - Histogram of the 21 plate scale values derived from the cross-correlation of the seven images of the theodolite cross-hairs. The dashed line represents the average value, and the gray region is the $1-\sigma$ uncertainty from the sample standard deviation of the measurements.

Finally, we convert the CM results from pixels units to arcseconds. Figure 5.13 shows the difference between the CMs as imaged by the right and left panels, in 
the $\mathrm{X}$ and $\mathrm{Y}$ axes of the focal plane, as a function of focus value. The figure also displays what measurement method was employed for each data point. There is a global trend between CM position and focus value along both axes. It seems that the behavior of the CM difference in this plane could be described by two straight lines, from $(\Delta X, \Delta Y) \sim(-13,5.2)$ to $(5,2)$ and then from $(5,2)$ to $(15,1.8)$. However, there is a group of data points that do not follow this trend, and correspond to values measured mostly with the image shifting method. In addition, there is one point $(-5,2.5)$ measured with the contour splitting method that lies right between the two groups of data points. Conversely, there are some data points that follow the main trend and that were measured with the contour splitting method too. Because of this behavior, we cannot conclude if the group of points off the main trend are erroneous measurements or not. In addition, the uncertainties for the image shifting method are around two or three pixels, i.e. 0.7-1.1 arcsec. Fortunately, the measurement for the best focus value follows the main trend. After applying the plate scale, we find a difference $\Delta(X, Y)=(2.6 \pm 0.7,2.3 \pm 0.7)$ arcsec between the spot produced by the right panel with respect to the left panel of the VPH.

The results that we obtained with the methodology presented here agree relatively well with the values derived by other, independent methods. In a report by Robert Barkhouser, he finds a difference of 2 arcseconds in the $\mathrm{X}$ direction. This result was obtained with an interferometric method of "tilt fringes" using the LUPI, the colimator, the VPH and a 20" flat mirror. In addition, John Wilson wrote a report with two more estimates, based on a third procedure. He uses the LUPI and the theodolite, but measures the shift angle directly from the angle change in a flat mirror. Both Barkhouser and Wilson estimated the shift angle, and found a difference of $3.2 \pm 0.5$ and $3.5 \pm 0.5$ arcseconds, respectively. Though our results are not within 


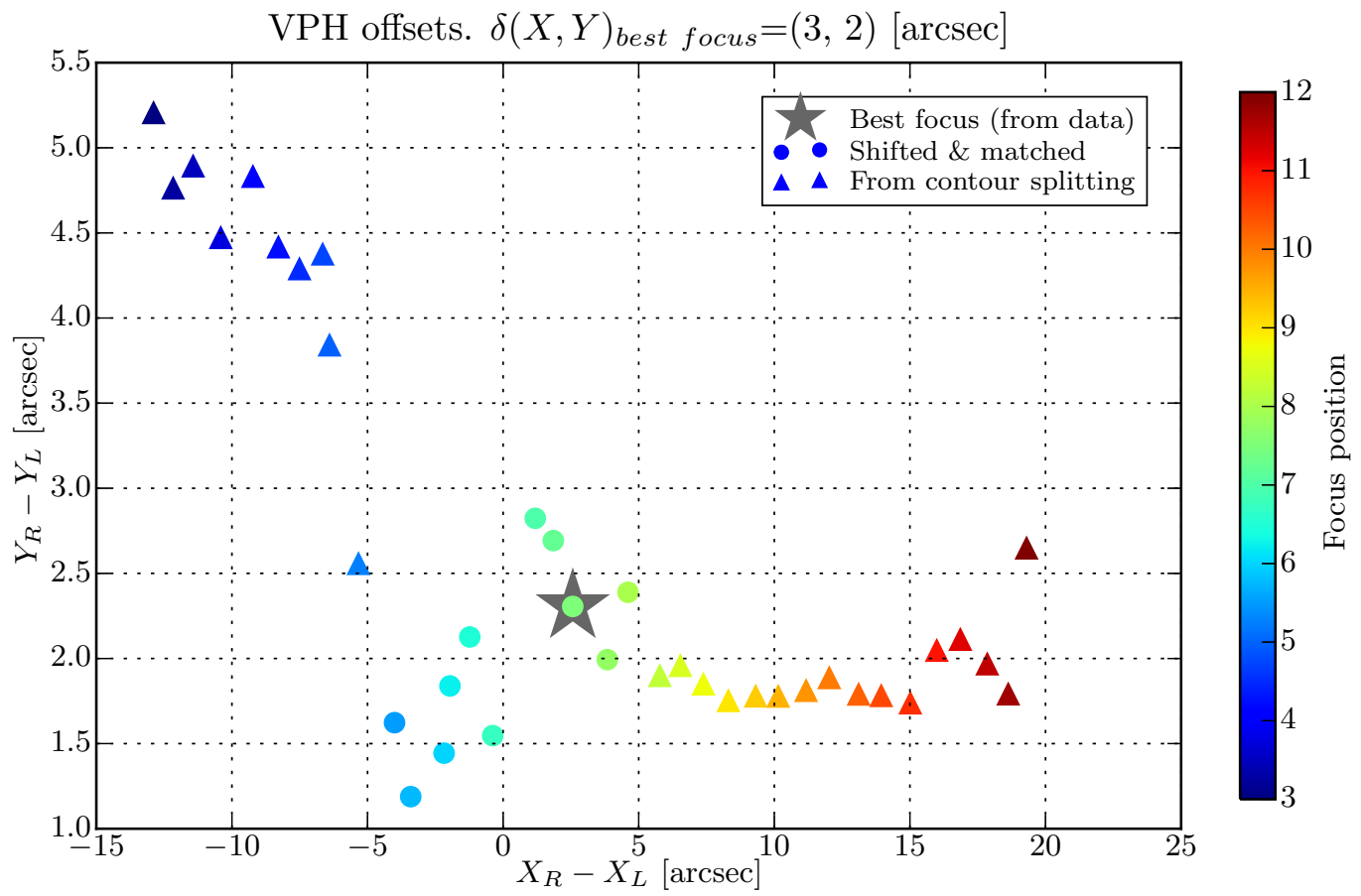

Fig. 5.13.- $-(\mathrm{X}, \mathrm{Y})$ shifts between the Center of Mass of the spots produced by the left and right sides of the VPH, color-coded by focus position. At best focus, we find a difference $\Delta(X, Y)=(2.6,2.3)$ arcseconds. The error for the the shifting and matching method is around two pixels in each direction, which translates into 0.7 arcseconds for a plate scale equal to $0.35 \mathrm{arcsec} / \mathrm{pixel}$.

the uncertainty claimed by the LUPI+theodolite method, our results are in between of the quantities estimated through the two other methods.

With the results from the three different methods in hand, John Wilson calculated that the VPH should produce a beam shift between 8.4 and $14.2 \operatorname{arcsec}$ for $\lambda=1.6 \mu \mathrm{m}$. Because the manufacturer (Kaiser Optical Systems, Inc.) claims that another VPH will not guarantee a mosaic with better characteristics, the APOGEE-2 instrument team is intending to install an optical corrector placed in front of one of the halves of the VPH. The three methods used to assess the VPH mosaic confirm, to good agreement, that a discrepancy between images resulting from each side of the VPH 
exists.

\subsection{The Plate Scale of the 2.54-m Irénée du Pont Telescope}

\subsubsection{Motivation}

The Irénée du Pont reflector, located at Las Campanas Observatory in northern Chile, has been selected as the telescope that will host the APOGEE-S spectrograph. This telescope possesses a $2.54 \mathrm{~m}$ diameter primary mirror, comparable to that of the Sloan Telescope (Gunn et al. 2006) that hosts the APOGEE-N instrument in New Mexico. Furthermore, both telescopes have large étendue. Although not as large as that of the Sloan Telescope (with a $3^{\circ}$ diameter FOV), the older du Pont delivers a very large field-of-view —with a diameter of $2.1^{\circ}$ that could be imaged with 20 " $\times 20$ " photographic plates - because "it was not planned to construct a companion instrument such as a large Schmidt for wide-angle surveys" ${ }^{2}$ by the Halo Observatories. Bowen \& Vaughan (1973) describe the optical design of this $\mathrm{f} / 7.5$ telescope in detail.

One of the key components for the success of APOGEE-S observations is the multiplexing capabilities provided by optical fibers. Such fibers collect light from multiple sources at the focal plane of the telescope and feed the light to the spectrograph. However, this approach requires precise positioning of the fiber ends at the telescope focal plane. To have a sense of the scales involved, consider the following example. Cudworth \& Rees (1991) measured a plate scale of $10.92 \mathrm{arcsec} / \mathrm{mm}$ for this telescope. For a FWHM of $\sim 1.0$ arcsec, the FWHM of a star at the focal plane

\footnotetext{
${ }^{2}$ Quoted from http://www.lco.cl/telescopes-information/irenee-du-pont
} 
is around $100 \mu \mathrm{m}$. Furthermore, Ritchey-Chrétien systems may exhibit third- and/or fifth-order radial distortions. These distortions change the plate scale as a function of radius from the optical axis of the system. Even though Cudworth \& Rees (1991) measured the scale and distortion constants of the du Pont, only measured them up to a 13 arcmin radius. APOGEE-S plans to exploit the full FOV of the du Pont, to a radius of a degree. Therefore, calculating precise values of the plate scale and radial distortions out to large radius becomes an essential exercise in planning the APOGEE-2S fiber cartridge system. For example, the solution obtained for the plate scale presented in this chapter have been successfully used for several engineering runs that have being carried out recently at the du Pont telescope. The purpose of these runs is to implement and test several subsystems of the telescope, in preparation for the spectrograph commissioning and survey operations. It would be impossible to execute these tests if the plate scale were not accurate enough to observe stars with optical fibers during the night.

Before we proceed, we give a brief overview of the method here to provide some context here. The basic idea of the method is to measure physical coordinates of a large number of stars, imaged on the focal plane of the telescope, and compare them against the positions obtained from a precise optical distortion model that converts the sky coordinates of the stars into focal plane coordinates. These sky coordinates are obtained from a precise astrometric catalog. Creating such a model requires us to solve for a transformation that converts from sky coordinates to focal plane physical coordinates. Such a transformation contains the plate scale as a function of radius from the optical axis on the focal plane. The radial distortions describe the change of plate scale with radius. Even though the concept is very simple, we had to consider several details that become mandatory to achieve the required precision in 
the transformation.

In the following sections, we describe the dataset and equipment involved in the observations. Next we describe the model. Finally, we present the results and actual on-sky verification of the results for some test plates.

\subsubsection{Data Acquisition and Reduction}

CCD images of stellar fields were obtained on the night of UT 2015-03-04 by members of the APOGEE-2 collaboration. The team mounted an SBIG STX-16803 CCD camera at the focal plane of the Cassegrain port of the du Pont telescope. The detector is a $4096 \times 4096$ array of square $9 \mu \mathrm{m}$ pixels. The array was used with $2 \times 2$ binning, giving a physical size equal to $36.8 \times 36.8 \mathrm{~mm}$. The camera was held in the focal plane with a mount called DAFFEE-2. This device is designed to simplify the adjustment of the position of the CCD camera along the focal plane in the radial direction. For this, the camera mount travels on a set of rails that work as tracks. The rails are curved along a radius of curvature $\sim 8,800 \mathrm{~mm}$ to account for the field curvature of the telescope focal plane. We need to know the radial position (with respect to the optical axis) of the camera along the focal plane to high accuracy. For this purpose, the DAFFEE-2 rails have nine drilled holes that allow us to fix the camera to specific radial positions on the rails. The locations of the holes (numbered from 0 to 8 ) is known to a precision of one hundredth of an inch, and are given in Table 5.1). Position 0 corresponds to the center of the focal plane, while position 8 is the outermost along the radial direction. We should remark that the uncertainty for the measured physical coordinates of the stars on the focal plane are required to be smaller than the uncertainty of the nine positions drilled on the DAFFEE. Nevertheless, we will account for this in the data modeling. 
At the telescope, the team collected a set of 23 sky images at the nine DAFFEE positions. Each image had an exposure time of 30 seconds, which was set to prevent saturation of the brightest stars in the field. The camera was equipped with an $I_{c}$ filter, and the detector edges were aligned to north and east on the sky. At the beginning of the observing sequence, the observers slewed the telescope close to the meridian, and at a declination of $\sim-40^{\circ}$, corresponding to a zenith distance for each image around $11^{\circ}$ (the latitude of LCO is $-29^{\circ}$ ). This choice was made to reduce the effects of telescope flexure and differential refraction on the astrometry, and because that region of the sky offered sufficient stars to obtain a good astrometric solution.

It would be convenient if CCD images from adjacent positions on the DAFFEE overlapped. In that case, we could match the stars common to a pair of overlapping images and measure precise physical positions on the focal plane because we know the pixel size. This approach just requires to keep the telescope tracking at sidereal rate - assuming the telescope does not drift - and only changing the position of the camera on the rails as we take images. Unfortunately, adjacent positions do not overlap because the CCD is smaller than the separation of the DAFEE-2 positions. To overcome this problem, the telescope was slewed between each change of position of the camera to ensure that adjacent fields would have some stars in common. However, this introduced a larger problem: the sky coordinates of the optical center is different for each image, and we cannot use the sky coordinates of the stars in the dataset to calculate the actual angular distance between contiguous fields from the astrometry. We address and solve this problem in the following section.

The collected images need to be processed and mapped to the World Coordinate System (WCS hereafter). The raw frames have enough quality such that they do not need to be bias corrected nor flat-fielded to get reliable astrometry. We performed the 
astrometric solution for each image with the astrometry. net blind astrometry solver software (Lang et al. 2010). We set the option to use SExtractor (Bertin \& Arnouts 1996) as the source identification and extraction algorithm because it outputs a file of $(x, y)$ pixel positions of the objects identified, along with a flag that specifies if these objects are likely to be stars. Then, we obtained a list of equatorial coordinates $(\alpha, \delta)$ for each $(x, y)$ entry using the WCS from astrometry.net. These equatorial coordinates are based on the WCS solution for the field, but are not necessarily the actual astrometric positions. Therefore, we matched these WCS-based positions to the astrometric data in UCAC-4 and 2MASS catalogs. In the end, we generated a catalog with $(x, y)$ image coordinates, in pixels, matched to celestial equatorial $(\alpha, \delta)$ coordinates. However, we still need to translate the image coordinates into physical focal plane coordinates, otherwise we cannot solve for the actual plate scale and distortions. This procedure will be described and executed in the next section.

\subsubsection{Data Modeling}

In this section, we present the model that we implemented to measure the plate scale and distortions.

We follow a methodology based on the work of Cudworth \& Rees (1991). In their work, they measured the (physical-) coordinates of stars on a photographic plate exposed on the du Pont, and then matched them to the positions of stars measured on a distortion-free "standard" plate of the same field. The standard plate is obtained from an astrograph, because the latter is a special, distortion-free optical system with well known plate scale, specially designed for astrometric measurements. One limitation of this method is that the field covered by the standard plate needs to be as large as the field of the system under calibration. After matching the stellar coordinates 
on both plates, the stars in the unknown plate are mapped to the known plate using a polynomial function by least-squares minimization. This function includes terms that correspond to the plate scale and distortions. Cudworth \& Rees made this fit independently for the $X$ and $Y$ axis of the plate, which was aligned along North-South direction.

The implementation of the Cudworth \& Rees method to our circumstances involves several steps, because we have to create a "synthetic" standard plate from an astrometric catalog. Though we describe them in detail later, the steps involved are listed here to give the reader a general view of the procedure. We:

1. Transform the celestial coordinates from the astrometric catalog, for all stars in each observed field, into observed (refraction-corrected) altitude-azimuth AltAz coordinates,

2. Convert the AltAz coordinates into a distortion-free "synthetic" standard plate. We aim to put all fields into a common coordinate reference frame, and then convert from this system into a system (in units of length) that describes the focal plane of the telescope accurately. The latter includes the desired terms for plate scale and distortion coefficients.

3. Convert the observed image coordinates - measured in pixels - for the same stars of the previous step into a common reference frame for the whole focal plane, and

4. Solve for the model parameters - which include plate scale and distortion coefficients - by least square minimization. We minimize the distance between the positions modeled from the "synthetic" catalog and the actual observed positions of stars on the focal plane. 
In the step (1) above, we convert the astrometric data from the matched catalogs -in the celestial equatorial frame - into the observer's AltAz system, using the routines from the Python Astropy.coordinates package. We performed this system conversion because we need to correct by atmospheric refraction, which would be the true observed coordinates at the telescope. The refraction model is that implemented in the Essential Routines for Fundamental Astronomy ERFA ${ }^{3}$ package. Refraction is modeled as $d Z=A \tan Z+B \tan ^{3} Z$, where $Z$ is the refracted zenith distance, and $d z$ is an additive correction that applied to $Z$ would result in the non-refracted zenith distance. The constants $A$ and $B$ depend on atmospheric conditions during the observation. We set the temperature, pressure and relative humidity to $12^{\circ} \mathrm{C}$, 750 mbar, and 50\%, respectively. The refraction model depends on the effective wavelength of observation, which corresponds to $8,060 \AA$ for the central wavelength of the $I_{c}$ filter. The most important effect of atmospheric refraction on the stellar positions, for our analysis, is differential refraction. This arises from the fact that stars within each CCD field have slightly different zenith distance. As a consequence, stars are refracted by a differential amount with respect to a mean refraction measured, for example, at the center of the field. For example, one of our observed field has an altitude of $78.44^{\circ}$ at its center, and extends for 7 arcmin in Altitude. The atmospheric refraction at this altitude is 8.92 arcsec, but the differential refraction is 0.10 arcsec. Although the last number may seem small, we seek uncertainties no larger than 0.30 arcsec. Thus, differential refraction can be a substantial source of systematic errors if not accounted for.

In the second step, we convert the refraction-corrected AltAz coordinates into the "synthetic" standard plate, and then into modeled focal plane coordinates through the plate scale and distortion constants. An important consideration in this step is

\footnotetext{
${ }^{3}$ https://github.com/liberfa/erfa
} 
that the distortion-free plate - as it would result from an astrograph - corresponds to a tangent-plane projection (gnomonic projection; Figure 5.14) (Green 1985).

The equations that define the projection are:

$$
\begin{aligned}
& \lambda=\frac{\cos \delta \sin \left(\alpha-\alpha_{p}\right)}{\sin \delta \sin \delta_{p}+\cos \delta \cos \delta_{p} \cos \left(\alpha-\alpha_{p}\right)}, \\
& \beta=\frac{\sin \delta \cos \delta_{p}-\cos \delta \sin \delta_{p} \cos \left(\alpha-\alpha_{p}\right)}{\sin \delta \sin \delta_{p}+\cos \delta \cos \delta_{p} \cos \left(\alpha-\alpha_{p}\right)},
\end{aligned}
$$

where $(\lambda, \beta)$ are angular coordinates, in radians, of a star with sky coordinates $(\alpha, \delta)$ projected onto a Cartesian tangent plane (Fig. 5.14). The coordinates of the tangent point between the plane and the sphere are $\left(\alpha_{p}, \delta_{p}\right)$, which will be projected at $(\lambda, \beta)=$ $(0,0)$. Because we do not know the coordinates $\left(\alpha_{p}, \delta_{p}\right)$ of the center of projection precisely, they need to be treated as model free parameters. An illustration of the projection is shown in Figure 5.14.

In addition, we include two rotations of the projection of each field imaged. The reason for this is twofold. First, the coordinate transformation from equatorial to AltAz coordinates introduces a rotation of the coordinate frame of the projection. This happens because the axes of the tangent projection are defined to coincide with the projection of the axes of the input sky coordinate system - in our case, the azimuth and altitude axes of each image. The angle between the orientation of the two coordinate systems is given by the parallactic angle $(q)$. Because all images were observed at different time of the night, the argument of the angle $q$ is different for all images. Thus, we need to correct for $q$ to put all images in a common coordinate system. Second, the sides of the CCD camera were mechanically aligned to follow the axes of the equatorial frame, but the orientation is not perfect. Because of this, we 


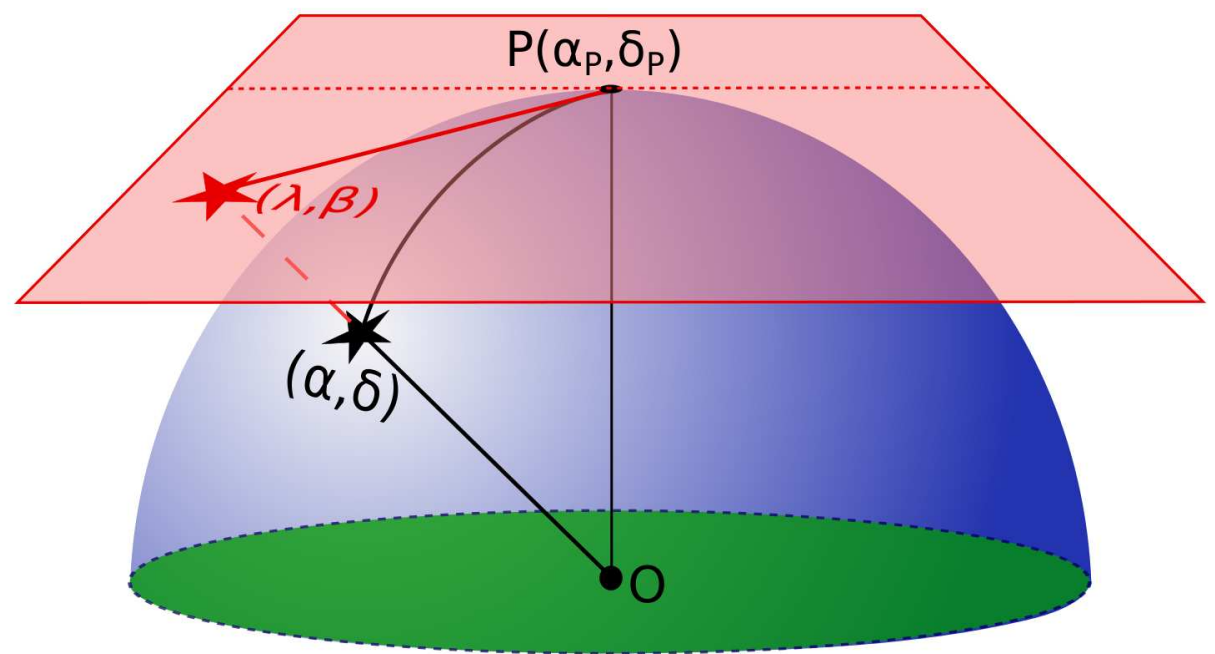

Fig. 5.14. - Illustration of a gnomonic or tangent plane projection. The plane is tangent to the sphere at point $P\left(\alpha_{p}, \delta_{p}\right)$, and a star with coordinates $(\alpha, \delta)$ will be projected at coordinates $(\lambda, \beta)$. The tangent point is projected onto the origin of the coordinate system of the plane. The observer is at point $\mathrm{O}$.

introduce a second rotation around a point close to the center of each field. These two rotations, shown in Figure 5.15, allow us to align all "standard" plates into a common reference frame. Both rotations are included in the model as free parameters. As a consequence, the projection for each field adds six free parameters to the model, because each rotation is defined by a center and the angle argument.

The projection and rotations performed will put the astrometric data of all images into a plane with a common coordinate system $\left(\lambda_{c}, \beta_{c}\right)$. This corresponds to a distortion free "standard" plate.

Then, we convert the angular units into length units that match the geometry of the focal plane. In this step we define the plate scale and distortion constants. We first transform the Cartesian coordinates into a polar frame defined as: 


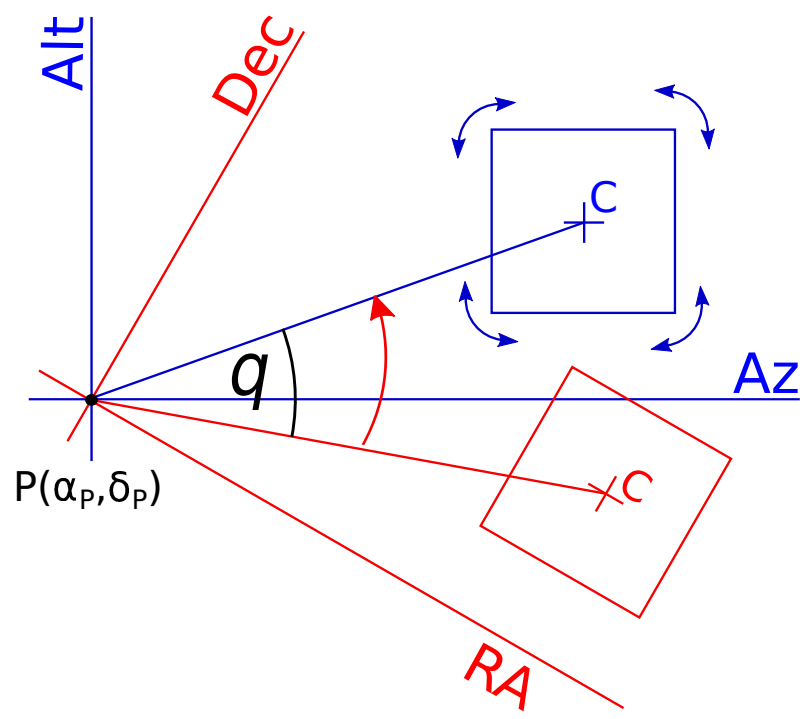

Fig. 5.15.- Illustration of the rotations applied to the gnomonic projection of each image. When we transform from equatorial (red) to AltAz (blue) coordinates, the frame is rotated by the parallactic angle $q$. We correct this effect with a first rotation around $P\left(\alpha_{P}, \delta_{P}\right)$. Then, a second rotation around point $C$ corrects for any small misalignment of the projected coordinates.

$$
\begin{aligned}
& \rho=\left(\lambda_{c}^{2}+\beta_{c}^{2}\right)^{1 / 2}, \\
& \theta=\arctan \frac{\beta_{c}}{\lambda_{c}},
\end{aligned}
$$

where $\rho$ and $\theta$ are the radial and angular components in radians, respectively. The transformation to units of length is:

$$
r_{\text {focal }}=s_{0} \rho+f(\rho)
$$

where $r_{\text {focal }}$ is the radial distance on the focal plane from the optical axis, in units of length. The quantity $s_{0}$ is the plate scale of the system, and $f(\rho)$ is a non-linear function that describes the radial distortions of the plate scale. Such distortions in Ritchey-Chrétien systems are well characterized by a third- and fifth-order terms. 
Thus,

$$
f(\rho)=s_{3} \rho^{3}+s_{5} \rho^{5},
$$

where coefficients of these terms, $s_{3}$ and $s_{5}$, are the distortion constants. Note that we do not need to convert the angular component $\theta$, because we only have radial distortions.

Finally, we convert the modeled focal plane positions from the polar frame back into a Cartesian system. The latter system matches the geometry of the CCD data better, and is what we use in the model minimization. The coordinates of stars, modeled from astrometric data, on the focal plane (in $\mathrm{mm}$ ) are:

$$
\begin{aligned}
& x_{f o c a l, a s t r}=r_{f o c a l} * \cos \theta, \\
& y_{f o c a l, a s t r}=r_{f o c a l} * \sin \theta
\end{aligned}
$$

In step (3), we convert the stellar positions $\left(x_{i m}, y_{i m}\right)$ - in pixels - for each image into a common coordinate system $\left(x_{f o c a l, i m}, y_{\text {focal }, i m}\right)$ that describes the focal plane, in millimeters and with the origin at the optical axis. To do so, we first transform the positions in pixel units, for each image, into millimeters $\left(x_{m}, y_{m}\right)$ by the binned pixel size of $18 \times 10^{-3} \mathrm{~mm}$. We set the origin of this system at the center of each image $\left(x_{o}, y_{o}\right)$. The origin for the common reference frame should be the optical axis, which should coincide with the center of the tangent projection. In addition, the center of the image in DAFFE's position 0 is almost aligned with the optical axis on purpose, and the camera is subsequently moved along the $Y$-axis to set it on positions $1-8$. Therefore, each field center is shifted in the $Y$-axis by an amount $y_{D A F F E E}$ given by the positions shown in Table 5.1. Thus, we convert star positions $\left(x_{m}, y_{m}\right)$, for each 
image, into the common focal-plane frame with equations:

$$
\begin{aligned}
& x_{f o c a l, i m}=x_{m}-x_{o}, \\
& y_{f o c a l, i m}=y_{m}-y_{o}-y_{D A F F E E}
\end{aligned}
$$

As we mentioned in the previous section, the actual location of the CCD in each DAFFEE-2 position is known to a limited precision. Hence, we set the origin of the coordinate system of each image $x_{o}, y_{o}+y_{D A F F E E}$ as free model parameters.

Finally, we solve for the model parameters described in the previous steps. We minimize the Euclidean distance between $\left(x_{f o c a l, a s t r}, y_{f o c a l, a s t r}\right)$ and $\left(x_{f o c a l, i m}, y_{f o c a l, i m}\right)$ (Equations 5.7 and 5.8, respectively). We need to solve for stars from images in the nine DAFEE-2 positions simultaneously. However, we do not know the telescope offsets applied between adjacent positions. Thus, we first minimize the model for one image in the position 0 , and use the solved parameters to estimate the field center of position 1 . Then we can solve for stars in images from position 0 and 1 simultaneously. We repeat this procedure for adding the next position into the solution until we solve the model for stars in the nine DAFEE-2 positions simultaneously. We select one image per DAFFEE-2 position from the dataset. We used the LMFIT library (Newville et al. 2014) in Python. This step solves for the plate-scale and distortion parameters, plus the six parameters for each field from the second step, and the two parameters for each field center as described in the third step. Since there are nine DAFFEE positions, the total number of free parameters adds up to 75 . These parameters are free to vary, but constrained within a minimum and maximum value that we set for each parameter individually.

The minimization uses the Nelder-Mead (Nelder \& Mead 1965) method, because 
we found that it converged for the large number of free parameters, while the most common Levenberg-Marquardt algorithm would not. The minimization requires initial values for the free parameters. Then, we set $x_{o}, y_{o}+y_{D A F F E E}$ to the known positions for the field centers on the DAFFEE-2 along the focal plane. The rotation values are set to the parallactic angle of the respective field center. The plate scale of the system is estimated by solving the model for the central field alone, and the value calculated by Cudworth \& Rees (1991). With these values, we guessed the initial parameters for the nine fields. We present results in the next section.

\subsubsection{Results and On-Sky Check}

We ran the minimization routine twice. First, we fit the 5-th order polynomial distortion, but found that term to be almost negligible $\left(s_{5} \sim 10^{-4}\left[\mathrm{~mm} / \mathrm{deg}^{5}\right]\right)$. Then, we fixed $s_{5}=0$ and ran the minimization a second time. Plate scale and distortion constants for both fits are presented in Table 5.2.

The residuals for the fit, using 665 stars in total, are $\sim 43 \mu \mathrm{m}$ in both cases. This value is slightly larger than our initial goal. Thus, we plotted the fit residuals to find clues on this (Figure 5.16). It can be readily seen that the residuals have a vortexlike pattern for all fields. In addition, the magnitude of the residuals increases with distance within each field; but the magnitude does not increase with distance from the optical axis. This means that the magnitude of the residuals increased because the model derived imprecise values for the rotation angles, but the values derived for the plate scale and distortion constants are acceptable.

The best test of our measurements comes from actual plates tested at the du Pont telescope in subsequent engineering runs. These runs are aimed at checking the telescope and implementing systems that will be required for APOGEE observations. 
Table 5.1. Positions for the CCD camera used in the analysis. The reference for the radial distance is the center of the focal plane, which is aligned to the optical axis.

\begin{tabular}{crr}
\hline \hline \multirow{2}{*}{ Position } & \multicolumn{2}{c}{ Radial distance } \\
\cline { 2 - 3 } & [Inches] & \multicolumn{1}{c}{$[\mathrm{mm}]$} \\
\hline 0 & 0.00 & 0.00 \\
1 & 1.64 & 41.65 \\
2 & 3.28 & 83.31 \\
3 & 4.92 & 124.97 \\
4 & 6.56 & 166.62 \\
5 & 8.20 & 208.28 \\
6 & 9.84 & 249.94 \\
7 & 11.48 & 291.59 \\
8 & 13.12 & 333.25 \\
\hline
\end{tabular}

Table 5.2. Solutions for the plate scale and distortion coefficients from the least-squares minimization. Two solutions are given, with and without the $s_{5}$ term.

\begin{tabular}{cccccccc}
\hline \hline Fit \# & $\begin{array}{c}s_{0} \\
{[\operatorname{arcsec} / \mathrm{mm}]}\end{array}$ & $\begin{array}{c}s_{0} \\
{[\mathrm{~mm} / \mathrm{deg}]}\end{array}$ & $\begin{array}{c}s_{3} \\
{\left[\mathrm{~mm} / \mathrm{deg}^{3}\right]}\end{array}$ & $\begin{array}{c}s_{5} \\
{\left[\mathrm{~mm} / \mathrm{deg}^{5}\right]}\end{array}$ & $\begin{array}{c}\text { RMS } \\
{[\mu \mathrm{m}]}\end{array}$ & N data & Free params. \\
\hline 1 & 10.9561 & 328.5828 & 2.0654 & $9.8107 \mathrm{E}-5$ & 43.2 & 665 & 75 \\
2 & 10.9559 & 328.5893 & 2.0748 & 0. (fixed) & 43.3 & 665 & 74 \\
\hline
\end{tabular}


For example, guiding during the observation of an APOGEE plate is accomplished using a set of coherent fibers, which do not scramble the spatial information of the incoming light. This guiding method at the du Pont requires precise positioning of fibers at the telescope focal plane, and is therefore sensitive to the plate scale and distortion constants of the optical system.

A set of four plates was drilled using the constants derived with our model. In addition, a second set of plates was drilled using the constants derived by S. Shectman for a fiber spectrograph that was in operation at the du Pont since 1989 (Shectman 1993). Paul Harding (Case Western Reserve University, OH) reported that the plates with our constants gave the best results in most situations, except for a field where Shectman's solution was better. The optimal plate position always was within $5 \mathrm{~mm}$ from the nominal focal plane position (set 8.75" below the telescope rotator). According to Harding, "the best RMS obtained for stars relative to guide fiber centers was 0.28 arcsec".

\subsection{Thermal Blanket Fabrication}

\subsubsection{Motivation}

There are important aspects of astronomical instrumentation that require operating at cryogenic temperatures. Most astronomical sources appear incredibly faint on the sky, therefore the number of photons collected by telescopes is often low. Photon counting is a statistical process, which carries some inherent uncertainty described by Poisson statistics. In addition, there are some inherent limitations in our instrumentation that adds extra noise to our data. Both depend on temperature. First, most astronomical CCD detectors are prone to dark current, which is the random gener- 
ation of electrons in the depletion region of each pixel (see Howell 2006). The rate of generation of these electrons is proportional to the CCD temperature. Unfortunately, these (unwanted) thermal electrons are indistinguishable from the (valuable) photo-electrons from astronomical sources. Thus, it is mandatory to decrease the temperature of the detector, which reduces dark current significantly. Second, nearIR instrumentation (even at $\lambda \sim 1.6 \mu m$ ) is sensitive to thermal radiation from the instrument enclosure. This drives the design of instruments to ensure that the detector "sees" cold surfaces in any direction to not swamp the photons from the input beam from the telescope. Thus, near IR instruments are designed to operate within a large cryostat.

Cryostats for near IR instrumentation, like APOGEE, are typically LN2 cooled. The APOGEE cryostat keeps a very low internal temperature $(\sim 77 K)$, by preventing the inflow of heat from the environment in two ways. First, the cryostat is under vacuum, otherwise molecules can transfer heat from the cryostat walls at room temperature. Second, the APOGEE cryostat is equipped with a "radiation shield", which consists of multi-layered insulation blankets (MLI). These blankets reduce the heat load by radiation on the interior. For instance, the APOGEE-N spectrograph is under a heat load of $\sim 30 \mathrm{~W}$ (Wilson et al. 2012). Because APOGEE-S is almost identical to APOGEE-N, we can expect a similar load.

Choi et al. (2013) present the theory that describe the physics behind MLI. We reproduce some of these equations here. The basic principle for MLI is that the heat exchange by radiation, $q$, per unit area between a pair of adjacent layers is balanced. For $N$ layers, the dissipated heat is:

$$
q=\frac{1}{N+1} \sigma\left[T_{\text {high }}^{4}-T_{\text {low }}^{4}\right]-\frac{1}{N+1} \varepsilon \sigma \sum_{i=1}^{N}\left[T_{i, t o p}^{4}-T_{i, \text { bottom }}^{4}\right]
$$


where $T_{\text {high }}$ and $T_{\text {low }}$ correspond to the temperatures of the surface in front of the most outermost (first) Mylar layer, and the surface facing the innermost Mylar layer, respectively. The terms $T_{i, t o p}$ and $T_{i, b o t t o m}$ represent the temperatures of the outer and inner surfaces of the $i-t h$ Mylar layer; whereas $\sigma$ and $\varepsilon$ are the Stephan-Boltzmann constant and the emissivity of the Mylar, respectively.

Within a layer of Mylar, the heat transfer - by conduction - follows the equation:

$$
q=\frac{\kappa}{d}\left[T_{i, t o p}-T_{i, \text { bottom }}\right]
$$

As Choi et al. (2013) point out, conventional MLI applications will have $T_{i, t o p}=$ $T_{i, \text { bottom }}$. Thus, equation 5.9 reduces to:

$$
q=\frac{1}{N+1} \sigma\left[T_{\text {high }}^{4}-T_{l o w}^{4}\right]
$$

This is usually referred to as the $N$-layer approximation (Rogatto 1993). Equation 5.11 shows that the heat load is inversely proportional to the number of layers.

\subsubsection{Design and Fabrication Process}

The technology and fabrication process of MLI blankets followed procedures learned on a 3-day training at the Pennsylvania State University, hosted by members of the team building "The Habitable-zone Planet Finder" (Mahadevan et al. 2012) spectrograph. These blanket fabrication techniques were then passed on to other members in the Virginia Astronomical Instrumentation Laboratory. In addition to the knowledge gained during the training at Penn-State, we applied some of the recommendations provided by NASA in its technical publication "Multilayer Insulation Material Guidelines" (Finckenor \& Dooling 1999). 
MLI blankets are fabricated with multiple layers of double-sided (0.25 mil thick) Mylar, spaced by bridal tulle. Mylar layers reflect the incoming IR radiation, while tulle layers are placed in between Mylar layers to work as a heat insulator and prevent heat flow by conduction between successive Mylar layers. Similar to MLI specifications for the APOGEE-N and HPF spectrographs, we fabricate blankets of $N=10$ layers (one layer comprises one Mylar plus one tulle layer). The blankets were built in three separate sections, that follow the design of the cryostat and aluminum radiation shield (Figure 5.17). These sections allow easy integration and future servicing of the instrument, if required. A top-blanket (or top-section) covers the upper section of the radiation shield. This section can be easily removed to access the optical bench where all optical components and detectors are mounted. A mid-blanket covers the mid-section of the radiation shield. This section is mounted during instrument integration and remains fixed. The bottom-blanket shields the bottom-section of the radiation shield. These bottom parts can be removed to gain access to the LN2 tank, if required.

Caution must be taken during blanket fabrication and mounting, otherwise the insulation capacity of the MLI can be decreased severely. There are a few simple but yet crucial guidelines. To begin with, it is extremely important that consecutive layers (i.e., the $i$-th and $i+1$-th) of Mylar do not to touch each other. Failing to do so will result in a heat short, allowing heat from an exterior layer to pass to an interior layer by conduction. At the same time, a single layer of MLI blanket may be made of several pieces of Mylar. In this case, the layer should be able to conduct heat throughout the layer to facilitate heat dissipation caused by an anisotropic heat load. Furthermore, at the union of two MLI blanket sections (for example, blankets from top and middle section) only the external Mylar layers can be in contact by 
overlap. To prevent thermal shorts across layers at the juncture, the edge of one section is folded inwards as a flap or cuff, and placed on top of the other section. Blanket placement has to be precise, otherwise the outermost Mylar layer from one section will be touching the innermost layer of the second section, and we would again introduce a heat short. Sections are usually secured to the aluminum radiation shield with a few 2-4-inches long strips of Velcro, which are sewn onto the edge of the blankets.

We introduced some new features in the design of the blankets for the APOGEES spectrograph compared to those for the APOGEE-N spectrograph. One of them, following the suggestion from the HPF team, is a technique for securing the Mylar+tulle layers together with plastic tagging pins (Hatakenaka et al. 2013). Such pins are widely used in clothing applications. This innovation translated in faster manufacturing and ease of blanket handling. For APOGEE-N blankets, a 10-layer blanket had to be carefully sewn before it could be manipulated. With the new approach, blanket layers are secured with the tagging pins quickly, and it can be manipulated right away. However, the Velcro strips must be sewn to the blankets.

Another innovation is a new design for blanket construction at the corners of the radiation shield. In the APOGEE-N instrument, MLI corners were built by sewing the two sides of the blanket right along the corner edge. The edge was covered with special tape after. However, this design presented some problems. First, NASA guidelines warn that any "slitting" (a cut along layers) can result in degraded blanket performance, because radiation can more easily pass through it. This type juncture may behave like a slit through the blanket if not done carefully. Second, any seaming on the blanket running right along an edge may create a protrusion, which needs to be avoided because the free room between the radiation shield and the cryostat wall 
is quite reduced. Finally, MYLAR is prone to tearing on stitches. In our new method, we design each blanket in such a way that we minimize all of these named problems.

To demonstrate the method, consider the upper section of the radiation shield, covered with a large single rectangular MLI blanket (Fig. 5.20). We do not trim the blanket along edges, but we fold the blanket around them. Because the angles in the lid are not right angles, we will end with an excess of blanket material at some places. To remove this excess material, we do some precise cuts on the blanket to remove some, but we keep around 2" where the folded blankets join and overlap. Then, we interleave the 2" of overlapping layers, paying special attention to prevent any heat shorts. Finally, the interleaved sections are secured with tagging pins.

The interleaving process requires gentle handling of the layers, because they cannot be secured until they are finished. The design requires solving for the geometry of the radiation shield section carefully, starting with a flat rectangular blanket. However, this presents another advantage because we can design the blankets to have the overlapping layers on a flat side. As a result, the APOGEE-S MLI blankets do not have cuts along corner edges.

We show the design of the blanket for the top-section in Figure 5.18.

Both lids require precise calculation of the angles where the blanket needs to be folded, trimmed or cut, which can be tedious. Figure 5.18 shows the design for the bottom section.

Pictures detailing the actual interleaving of the bottom-section blanket are shown in Figure 5.20.

One can ask the question on how effective this solution is for heat insulation. We see that for each layer of Mylar, we place the top surface from one strip in contact with the bottom surface of the other strip. However, we think this is a safe decision 
because it is assumed that $T_{i, \text { bottom }}=T_{i, \text { top }}$, i.e., the whole Mylar layer is at the same temperature and there is no heat gradient through the layer. The layer thickness is only 0.25 mil $(6 \mu m)$.

Another aspect that we checked is the layer separation. Rogatto (1993) recommends a layer density of 30 layers per centimeter. APOGEE-S is close to optimal, because ten layers are held by $3.5 \mathrm{~mm}$ pins.

Several IR-lab members and visitors participated in the construction of the blankets: Mita Tembe, Sophia Bruner (visiting former lab member), Juan Pablo Colque (visiting from Universidad de Antofagasta, Chile), Matthew Hall, and Garrett Ebelke. 


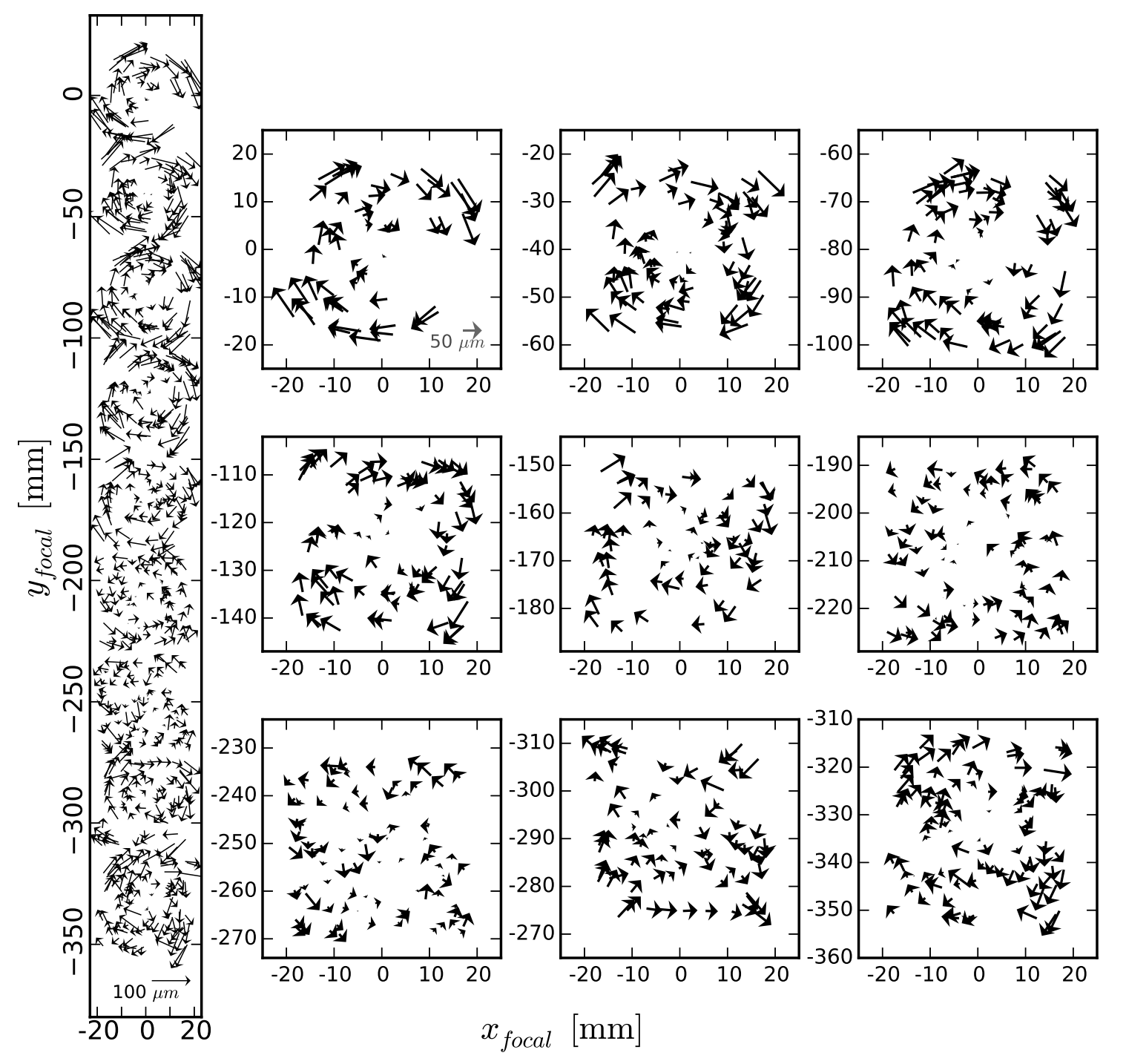

Fig. 5.16. - The residuals of the solution for model \#1. Vectors illustrate the direction and magnitude of the residuals, $\left(x_{\text {focal }, \text { astr }}-x_{\text {focal }, \text { im }}, y_{\text {focal }, \text { astr }}-y_{\text {focal }, \text { im }}\right)$. The long strip on the left displays the nine fields along DAFFEE-2 on the focal plane; meanwhile the square panels on the right show the data used for the individual positions. All panels have the axes with a 1:1 aspect ratio. The scale for the length of the vectors is indicated by an arrow representing $100 \mu \mathrm{m}$ and $50 \mu \mathrm{m}$ residuals for the rectangular and square plots, respectively. Note the rotation pattern for most of the fields. We think this is not a problem with the derived distortion constants or scale, because the magnitude of the residuals does not change dramatically with radius from the optical axis. 


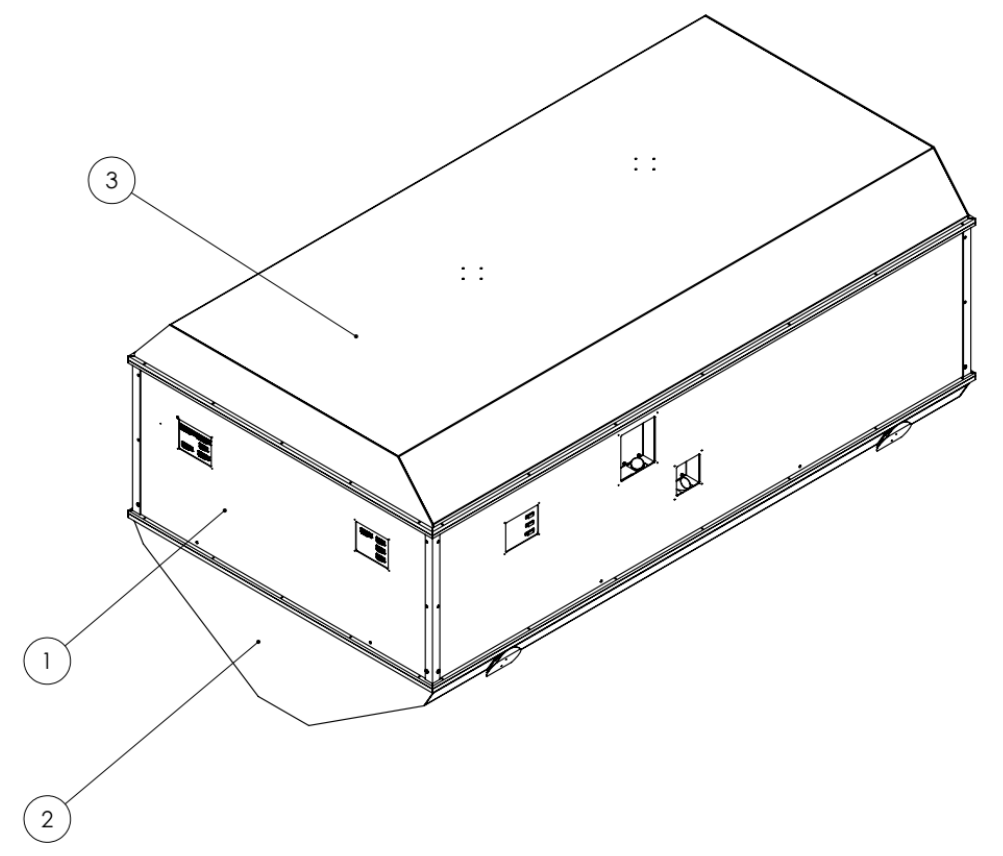

Fig. 5.17.- Isometric view of the APOGEE-S radiation shield. The numbers indicate the (1) mid-section, (2) bottom-section, and (3) top-section. The whole radiation shield is enclosed by the cryostat. Figure courtesy of Garrett L. Ebelke (UVA). 

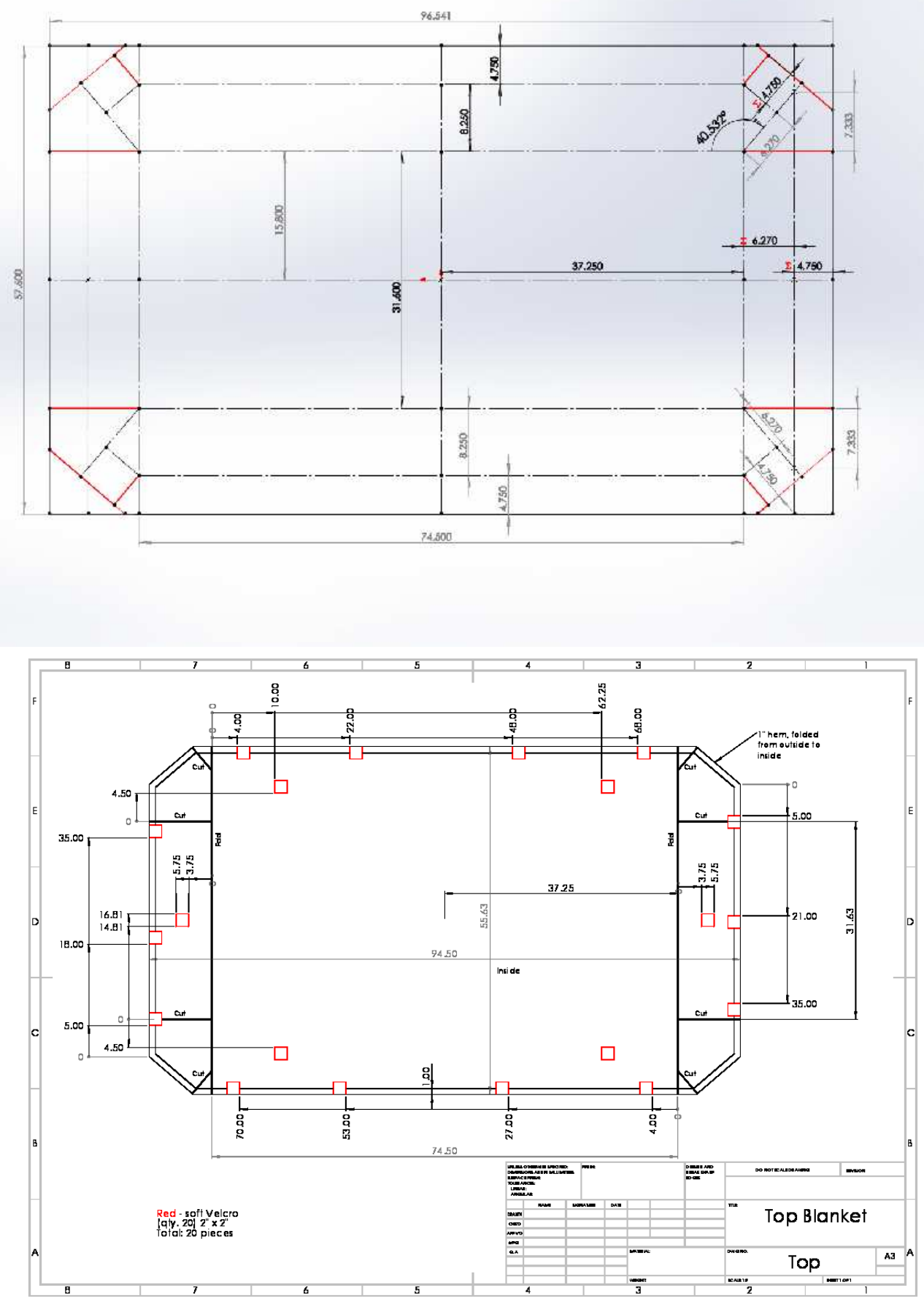

Fig. 5.18. - Design of the top-section MLI blanket. Top: the blanket design requires precise calculation of the lid geometry, and then calculate angles and required cuts (in red). Bottom: The shape of trimmed blanket, along with the required cuts. Drawings courtesy of Mita Tembe (UVA) and Juan Pable Colque (U. Antofagasta). 

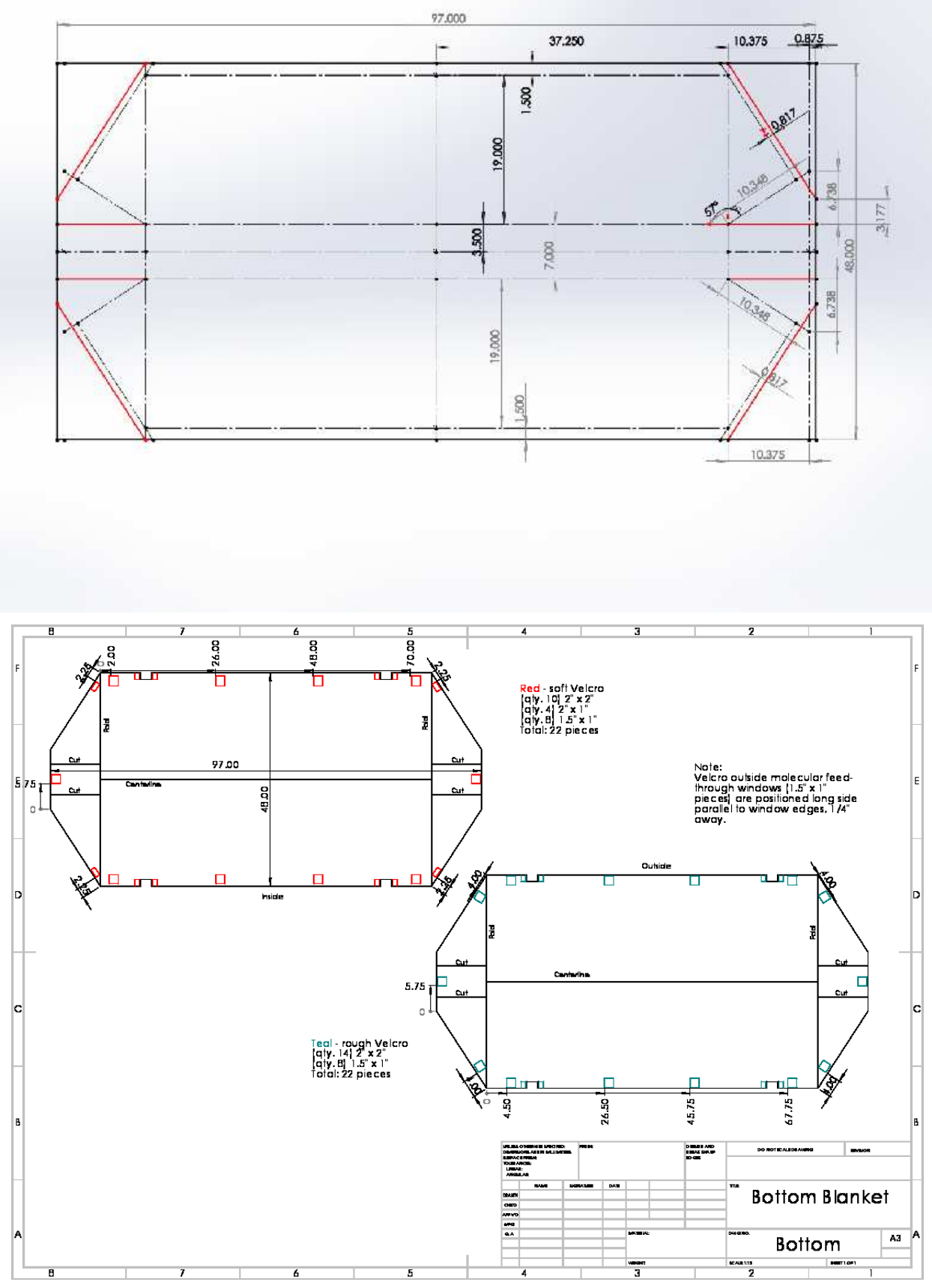

Fig. 5.19. - Same as figure 5.18, but for the bottom-section blanket. Drawings courtesy of Mita Tembe (UVA) and Juan Pable Colque (U. Antofagasta). 

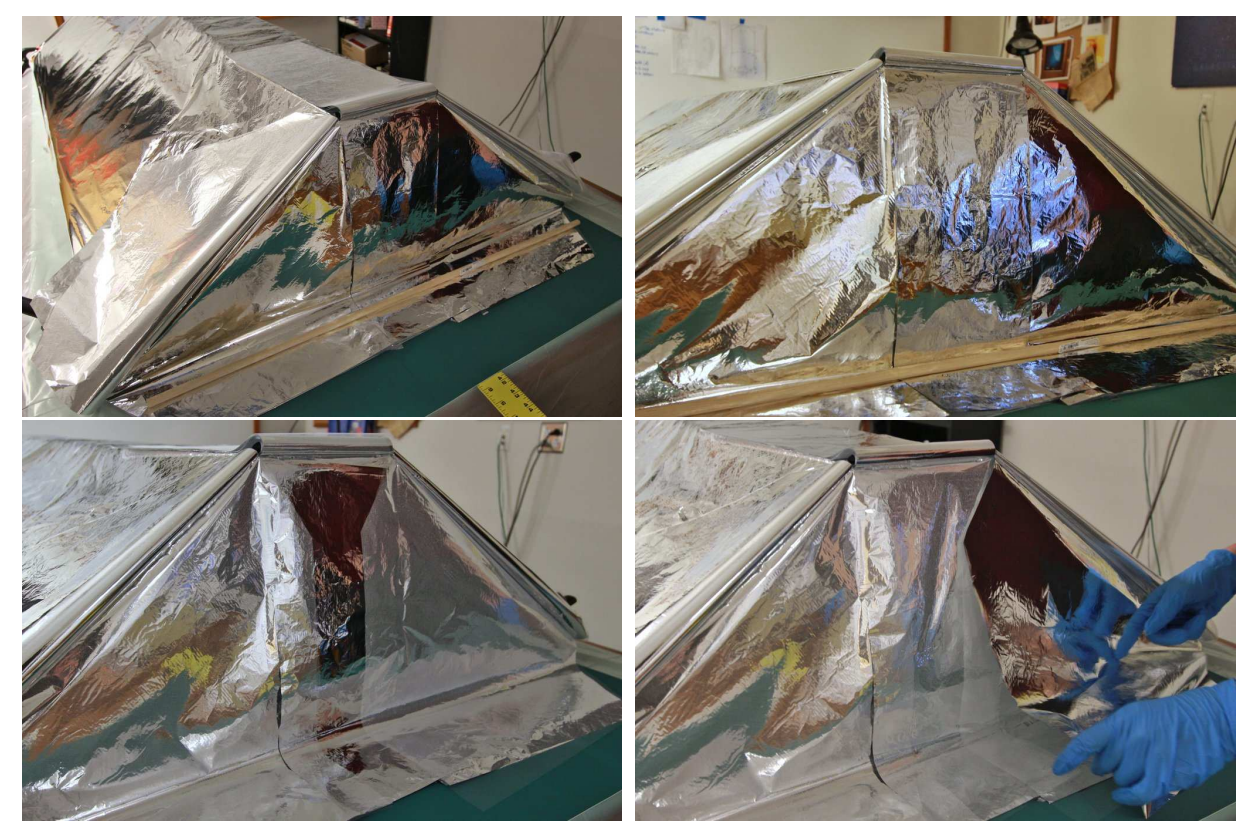

Fig. 5.20.- The layer interleaving technique for the fabrication of the bottom-section MLI blanket. Upper left: a general view of one side of the blanket under fabrication. The tulle and Mylar strips of the subsequent layers can be seen folded on top of the mock-up radiation shield built to fabricate the blankets. Upper right: the lateral layer of Mylar is the result of three overlapping strips. Lower left: two strips of the subsequent tulle layer unfolded. The two tulle flaps that form the overlapping region can be seen in the center. Next, the tulle strip that covers the exposed Mylar in the center will be unfolded form the top. Lower right: One strip of the next layer of Mylar is unfolded. The completed tulle layer is seen underneath, together with the overlapping tulle flaps. Mylar and tulle layers are cut to the same shape. Once completed, all layers are secured together with tagging pins, placed along the two strips of interleaved layers. Therefore, there are no seams along edges, nor slit-like junctures. 


\section{Chapter 6}

\section{Summary and Future Work}

In this thesis, we have made extensive use of stellar spectra of late type giant stars to study dynamics and chemistry of stellar populations in the MW.

We exploited the near-IR Ca II triplet to measure radial velocities and estimate metallicities along the tails of the Sgr dwarf galaxy for a sample of M-giants. We have found a group of four likely-Sgr stars that deviate from the known on-sky location of the main branch of the leading arm of Sgr. These stars have radial velocities and magnitudes consistent with Sgr tidal debris. We interpret the deviation of these stars as a result of the precession of Sgr tidal debris within an oblate dark matter halo. According to N-body simulations, finding Sgr stars in the deviation requires a more oblate halo. For future work, we plan to use the sample of stars presented here to update the Law \& Majewski (2010) model.

We presented a new method to derive stellar metallicities based on the traditional calcium triplet method. However, the method that we propose can be applied to stars with unknown distances, which certainly provides a new tool to measure stellar metallicities in RGB stars. This new method can be used for stars with unknown distances, which certainly provides a new tool to measure stellar metallicities in RGB 
stars. We applied the modified calcium triplet method to estimate metallicities of Sgr stars along the tidal tails. We found evidence of a metallicity gradient along each tidal tail, in agreement with studies carried out by other authors. For future work, we plan to test this method as an estimator of reddening towards clusters.

We derived preliminary results of Solar motion and the LSR using K-giant stars in the Grid Giant Star Survey. We carried out simulations to estimate that the $U_{\odot}$ and $W_{\odot}$ components of the Solar motion are mostly stable under distance and velocity uncertainty. However, the LSR circular velocity and the $V_{\odot}$ components are largely affected by uncertainties. We will continue improving the model and characterizing the GGSS, which should reduce the impact of uncertainties on the derived parameters.

We presented part of my contributions to the construction of the APOGEE-2S spectrograph. First, we developed a method to measure the angular mismatch between the output beams of the candidate VPH disperser. We found shifts between the output beams of the VPH panels, along both the spectral and the spatial directions. Next, we determined the plate scale and radial distortions of the 2.54-m Irénée du Pont telescope to a radius of one degree. For this, we developed a relatively complex model to account for the discontinuous radial sampling of the data collected at the focal plane. Finally, we presented a new method for fabricating corners of thermal MLI blankets. We expect this method to improve the insulation capabilities of the MLI blankets, compared to the original APOGEE spectrograph. 


\section{References}

Arias, E. F., Charlot, P., Feissel, M., \& Lestrade, J.-F. 1995, A\&A, 303, 604

Armandroff, T. E. \& Da Costa, G. S. 1991, AJ, 101, 1329

Armandroff, T. E. \& Zinn, R. 1988, AJ, 96, 92

Arns, J., Wilson, J. C., Skrutskie, M., Smee, S., Barkhouser, R., Eisenstein, D., Gunn, J., Hearty, F., Harding, A., Maseman, P., Holtzman, J., Schiavon, R., Gillespie, B., \& Majewski, S. 2010, in Proc. SPIE, Vol. 7739, Modern Technologies in Spaceand Ground-based Telescopes and Instrumentation, 773913

Belokurov, V., Zucker, D. B., Evans, N. W., Gilmore, G., Vidrih, S., Bramich, D. M., Newberg, H. J., Wyse, R. F. G., Irwin, M. J., Fellhauer, M., Hewett, P. C., Walton, N. A., Wilkinson, M. I., Cole, N., Yanny, B., Rockosi, C. M., Beers, T. C., Bell, E. F., Brinkmann, J., Ivezić, Ž., \& Lupton, R. 2006, ApJ, 642, L137

Bertin, E. \& Arnouts, S. 1996, A\&AS, 117, 393

Binney, J. \& Merrifield, M. 1998, Galactic Astronomy

Boeche, C. \& Grebel, E. K. 2016, A\&A, 587, A2

Bowen, I. S. \& Vaughan, Jr., A. H. 1973, Appl. Opt., 12, 1430 
Bressan, A., Marigo, P., Girardi, L., Salasnich, B., Dal Cero, C., Rubele, S., \& Nanni, A. 2012, MNRAS, 427, 127

Burstein, D., Faber, S. M., Gaskell, C. M., \& Krumm, N. 1984, ApJ, 287, 586

Cardelli, J. A., Clayton, G. C., \& Mathis, J. S. 1989, ApJ, 345, 245

Carlin, J. L., Majewski, S. R., Casetti-Dinescu, D. I., Law, D. R., Girard, T. M., \& Patterson, R. J. 2012, ApJ, 744, 25

Carrera, R., Gallart, C., Pancino, E., \& Zinn, R. 2007, AJ, 134, 1298

Carrera, R., Pancino, E., Gallart, C., \& del Pino, A. 2013, MNRAS, 434, 1681

Carretta, E. \& Gratton, R. G. 1997, A\&AS, 121, 95

Cenarro, A. J., Cardiel, N., Gorgas, J., Peletier, R. F., Vazdekis, A., \& Prada, F. 2001, MNRAS, 326, 959

Cenarro, A. J., Peletier, R. F., Sánchez-Blázquez, P., Selam, S. O., Toloba, E., Cardiel, N., Falcón-Barroso, J., Gorgas, J., Jiménez-Vicente, J., \& Vazdekis, A. 2007, MNRAS, 374, 664

Choi, J., Ishitsuka, H., Mima, S., Oguri, S., Takahashi, K., \& Tajima, O. 2013, Review of Scientific Instruments, 84, 114502

Chou, M.-Y., Majewski, S. R., Cunha, K., Smith, V. V., Patterson, R. J., MartínezDelgado, D., Law, D. R., Crane, J. D., Muñoz, R. R., Garcia López, R., Geisler, D., \& Skrutskie, M. F. 2007, ApJ, 670, 346

Cole, A. A., Smecker-Hane, T. A., Tolstoy, E., Bosler, T. L., \& Gallagher, J. S. 2004, MNRAS, 347, 367 
Crane, J. D., Majewski, S. R., Patterson, R. J., Skrutskie, M. F., Adams, E. Y., \& Frinchaboy, P. M. 2005, PASP, 117, 526

Cudworth, K. M. \& Rees, R. F. 1991, PASP, 103, 470

Cui, X.-Q., Zhao, Y.-H., Chu, Y.-Q., Li, G.-P., Li, Q., Zhang, L.-P., Su, H.-J., Yao, Z.-Q., Wang, Y.-N., Xing, X.-Z., Li, X.-N., Zhu, Y.-T., Wang, G., Gu, B.-Z., Luo, A.-L., Xu, X.-Q., Zhang, Z.-C., Liu, G.-R., Zhang, H.-T., Yang, D.-H., Cao, S.-Y., Chen, H.-Y., Chen, J.-J., Chen, K.-X., Chen, Y., Chu, J.-R., Feng, L., Gong, X.-F., Hou, Y.-H., Hu, H.-Z., Hu, N.-S., Hu, Z.-W., Jia, L., Jiang, F.-H., Jiang, X., Jiang, Z.-B., Jin, G., Li, A.-H., Li, Y., Li, Y.-P., Liu, G.-Q., Liu, Z.-G., Lu, W.-Z., Mao, Y.-D., Men, L., Qi, Y.-J., Qi, Z.-X., Shi, H.-M., Tang, Z.-H., Tao, Q.-S., Wang, D.-Q., Wang, D., Wang, G.-M., Wang, H., Wang, J.-N., Wang, J., Wang, J.-L., Wang, J.-P., Wang, L., Wang, S.-Q., Wang, Y., Wang, Y.-F., Xu, L.-Z., Xu, Y., Yang, S.-H., Yu, Y., Yuan, H., Yuan, X.-Y., Zhai, C., Zhang, J., Zhang, Y.-X., Zhang, Y., Zhao, M., Zhou, F., Zhou, G.-H., Zhu, J., \& Zou, S.-C. 2012, Research in Astronomy and Astrophysics, 12, 1197

Debattista, V. P., Roškar, R., Valluri, M., Quinn, T., Moore, B., \& Wadsley, J. 2013, MNRAS, 434, 2971

Deg, N. \& Widrow, L. 2014, MNRAS, 439, 2678

Dehnen, W. \& Binney, J. J. 1998, MNRAS, 298, 387

Dotter, A., Chaboyer, B., Jevremović, D., Kostov, V., Baron, E., \& Ferguson, J. W. 2008, ApJS, 178, 89

Finckenor, M. \& Dooling, D. 1999, Multilayer Insulation Material Guidelines, NASA technical paper (NASA Marshall Space Flight Center) 
Fischer, D. A. \& Valenti, J. 2005, ApJ, 622, 1102

Francis, C. \& Anderson, E. 2009, Proceedings of the Royal Society A: Mathematical, Physical and Engineering Sciences, 14, 615

—. 2014, Celestial Mechanics and Dynamical Astronomy, 118, 399

Friel, E. D., Janes, K. A., Tavarez, M., Scott, J., Katsanis, R., Lotz, J., Hong, L., \& Miller, N. 2002, AJ, 124, 2693

Frinchaboy, P. M. \& Majewski, S. R. 2008, AJ, 136, 118

Frinchaboy, P. M., Majewski, S. R., Muñoz, R. R., Law, D. R., Łokas, E. L., Kunkel, W. E., Patterson, R. J., \& Johnston, K. V. 2012, ApJ, 756, 74

Geisler, D. 1984, PASP, 96, 723

Gilbert, K. M., Guhathakurta, P., Kalirai, J. S., Rich, R. M., Majewski, S. R., Ostheimer, J. C., Reitzel, D. B., Cenarro, A. J., Cooper, M. C., Luine, C., \& Patterson, R. J. 2006, ApJ, 652, 1188

Gómez, F. A., Besla, G., Carpintero, D. D., Villalobos, Á., O’Shea, B. W., \& Bell, E. F. 2015, ApJ, 802, 128

Green, R. M. 1985, Spherical astronomy

Gunn, J. E., Siegmund, W. A., Mannery, E. J., Owen, R. E., Hull, C. L., Leger, R. F., Carey, L. N., Knapp, G. R., York, D. G., Boroski, W. N., Kent, S. M., Lupton, R. H., Rockosi, C. M., Evans, M. L., Waddell, P., Anderson, J. E., Annis, J., Barentine, J. C., Bartoszek, L. M., Bastian, S., Bracker, S. B., Brewington, H. J., Briegel, C. I., Brinkmann, J., Brown, Y. J., Carr, M. A., Czarapata, P. C., Drennan, C. C., Dombeck, T., Federwitz, G. R., Gillespie, B. A., Gonzales, C., 
Hansen, S. U., Harvanek, M., Hayes, J., Jordan, W., Kinney, E., Klaene, M., Kleinman, S. J., Kron, R. G., Kresinski, J., Lee, G., Limmongkol, S., Lindenmeyer, C. W., Long, D. C., Loomis, C. L., McGehee, P. M., Mantsch, P. M., Neilsen, Jr., E. H., Neswold, R. M., Newman, P. R., Nitta, A., Peoples, Jr., J., Pier, J. R., Prieto, P. S., Prosapio, A., Rivetta, C., Schneider, D. P., Snedden, S., \& Wang, S.-i. 2006, AJ, 131, 2332

Harris, W. E. 1996, AJ, 112, 1487

Hatakenaka, R., Miyakita, T., Sugita, H., Saitoh, M., \& Hirai, T. 2013, in 43RD International Conference on Environmental Systems (American Institute of Aeronautics and Astronautics)

Howell, S. B. 2006, Handbook of CCD Astronomy, ed. R. Ellis, J. Huchra, S. Kahn, G. Rieke, \& P. B. Stetson

Ibata, R. A., Gilmore, G., \& Irwin, M. J. 1994, Nature, 370, 194

Ibata, R. A. \& Lewis, G. F. 1998, ApJ, 500, 575

Indebetouw, R., Mathis, J. S., Babler, B. L., Meade, M. R., Watson, C., Whitney, B. A., Wolff, M. J., Wolfire, M. G., Cohen, M., Bania, T. M., Benjamin, R. A., Clemens, D. P., Dickey, J. M., Jackson, J. M., Kobulnicky, H. A., Marston, A. P., Mercer, E. P., Stauffer, J. R., Stolovy, S. R., \& Churchwell, E. 2005, ApJ, 619, 931

Johnston, K. V. 1998, ApJ, 495, 297

Johnston, K. V., Bullock, J. S., Sharma, S., Font, A., Robertson, B. E., \& Leitner, S. N. 2008, ApJ, 689, 936

Johnston, K. V., Hernquist, L., \& Bolte, M. 1996, ApJ, 465, 278 
Johnston, K. V., Law, D. R., \& Majewski, S. R. 2005, ApJ, 619, 800

Johnston, K. V., Spergel, D. N., \& Hernquist, L. 1995, ApJ, 451, 598

Jones, E. M. 1972, ApJ, 173, 671

Jones, J. E., Alloin, D. M., \& Jones, B. J. T. 1984, ApJ, 283, 457

Koposov, S. E., Belokurov, V., Evans, N. W., Gilmore, G., Gieles, M., Irwin, M. J., Lewis, G. F., Niederste-Ostholt, M., Peñarrubia, J., Smith, M. C., Bizyaev, D., Malanushenko, E., Malanushenko, V., Schneider, D. P., \& Wyse, R. F. G. 2012, ApJ, 750, 80

Lang, D., Hogg, D. W., Mierle, K., Blanton, M., \& Roweis, S. 2010, AJ, 139, 1782

Law, D. R., Johnston, K. V., \& Majewski, S. R. 2005, ApJ, 619, 807

Law, D. R. \& Majewski, S. R. 2010, ApJ, 714, 229

Law, D. R., Majewski, S. R., \& Johnston, K. V. 2009, ApJ, 703, L67

Law, D. R., Majewski, S. R., Skrutskie, M. F., \& Johnston, K. V. 2004, in Astronomical Society of the Pacific Conference Series, Vol. 327, Satellites and Tidal Streams, ed. F. Prada, D. Martinez Delgado, \& T. J. Mahoney, 239

Lee, T. A. 1970, ApJ, 162, 217

Lee, Y.-W., Demarque, P., \& Zinn, R. 1990, ApJ, 350, 155

Lépine, S., Shara, M. M., \& Rich, R. M. 2003, AJ, 126, 921

Luyten, W. J. 1922, Lick Observatory Bulletin, 10, 135 
Mahadevan, S., Ramsey, L., Bender, C., Terrien, R., Wright, J. T., Halverson, S., Hearty, F., Nelson, M., Burton, A., Redman, S., Osterman, S., Diddams, S., Kasting, J., Endl, M., \& Deshpande, R. 2012, in Proc. SPIE, Vol. 8446, Ground-based and Airborne Instrumentation for Astronomy IV, 84461S

Majewski, S. R., Kunkel, W. E., Law, D. R., Patterson, R. J., Polak, A. A., RochaPinto, H. J., Crane, J. D., Frinchaboy, P. M., Hummels, C. B., Johnston, K. V., Rhee, J., Skrutskie, M. F., \& Weinberg, M. 2004, AJ, 128, 245

Majewski, S. R., Nidever, D. L., Smith, V. V., Damke, G. J., Kunkel, W. E., Patterson, R. J., Bizyaev, D., \& García Pérez, A. E. 2012, ApJ, 747, L37

Majewski, S. R., Ostheimer, J. C., Kunkel, W. E., \& Patterson, R. J. 2000, AJ, 120, 2550

Majewski, S. R., Schiavon, R. P., Frinchaboy, P. M., Allende Prieto, C., Barkhouser, R., Bizyaev, D., Blank, B., Brunner, S., Burton, A., Carrera, R., Chojnowski, S. D., Cunha, K., Epstein, C., Fitzgerald, G., Garcia Perez, A. E., Hearty, F. R., Henderson, C., Holtzman, J. A., Johnson, J. A., Lam, C. R., Lawler, J. E., Maseman, P., Meszaros, S., Nelson, M., Coung Nguyen, D., Nidever, D. L., Pinsonneault, M., Shetrone, M., Smee, S., Smith, V. V., Stolberg, T., Skrutskie, M. F., Walker, E., Wilson, J. C., Zasowski, G., Anders, F., Basu, S., Beland, S., Blanton, M. R., Bovy, J., Brownstein, J. R., Carlberg, J., Chaplin, W., Chiappini, C., Eisenstein, D. J., Elsworth, Y., Feuillet, D., Fleming, S. W., Galbraith-Frew, J., Garcia, R. A., Anibal Garcia-Hernandez, D., Gillespie, B. A., Girardi, L., Gunn, J. E., Hasselquist, S., Hayden, M. R., Hekker, S., Ivans, I., Kinemuchi, K., Klaene, M., Mahadevan, S., Mathur, S., Mosser, B., Muna, D., Munn, J. A., Nichol, R. C., O'Connell, R. W., Robin, A. C., Rocha-Pinto, H., Schultheis, M., Serenelli, A. M., Shane, N., 
Silva Aguirre, V., Sobeck, J. S., Thompson, B., Troup, N. W., Weinberg, D. H., \& Zamora, O. 2015, ArXiv e-prints

Majewski, S. R., Skrutskie, M. F., Weinberg, M. D., \& Ostheimer, J. C. 2003, ApJ, 599,1082

Majewski, S. R., Zasowski, G., \& Nidever, D. L. 2011, ApJ, 739, 25

Mauro, F., Moni Bidin, C., Geisler, D., Saviane, I., Da Costa, G. S., GormazMatamala, A. C., Vasquez, S., Chené, A.-N., Cohen, R., \& Dias, B. 2014, A\&A, $563, \mathrm{~A} 76$

Merrifield, M. R. 1992, AJ, 103, 1552

Monet, D. G., Levine, S. E., Canzian, B., Ables, H. D., Bird, A. R., Dahn, C. C., Guetter, H. H., Harris, H. C., Henden, A. A., Leggett, S. K., Levison, H. F., Luginbuhl, C. B., Martini, J., Monet, A. K. B., Munn, J. A., Pier, J. R., Rhodes, A. R., Riepe, B., Sell, S., Stone, R. C., Vrba, F. J., Walker, R. L., Westerhout, G., Brucato, R. J., Reid, I. N., Schoening, W., Hartley, M., Read, M. A., \& Tritton, S. B. 2003, AJ, 125, 984

Navarro, J. F., Frenk, C. S., \& White, S. D. M. 1996, ApJ, 462, 563

Nelder, J. A. \& Mead, R. 1965, The Computer Journal, 7, 308

Newville, M., Stensitzki, T., Allen, D. B., \& Ingargiola, A. 2014, LMFIT: Non-Linear Least-Square Minimization and Curve-Fitting for Python

Nidever, D. L., Zasowski, G., \& Majewski, S. R. 2012, ApJS, 201, 35

Olszewski, E. W., Schommer, R. A., Suntzeff, N. B., \& Harris, H. C. 1991, AJ, 101, 515 
Patterson, R. J., Majewski, S. R., Slesnick, C. L., Rhee, J., Crane, J. D., Polak, A. A., Kundu, A., Kunkel, W. E., Geisler, D., Munoz, R., Arenas, J., Seguel, J., Gieren, W., Smith, V. V., Benedict, G. F., \& Johnston, K. V. 2001, in Astronomical Society of the Pacific Conference Series, Vol. 246, IAU Colloq. 183: Small Telescope Astronomy on Global Scales, ed. B. Paczynski, W.-P. Chen, \& C. Lemme, 65

Peñarrubia, J., Belokurov, V., Evans, N. W., Martínez-Delgado, D., Gilmore, G., Irwin, M., Niederste-Ostholt, M., \& Zucker, D. B. 2010, MNRAS, 408, L26

Prugniel, P., Vauglin, I., \& Koleva, M. 2011, A\&A, 531, A165

Read, J. I. 2014, Journal of Physics G Nuclear Physics, 41, 063101

Roeser, S., Demleitner, M., \& Schilbach, E. 2010, AJ, 139, 2440

Rogatto, W. D. 1993, Electro-optical components

Rutledge, G. A., Hesser, J. E., \& Stetson, P. B. 1997, PASP, 109, 907

Schiavon, R. P., Barbuy, B., Rossi, S. C. F., Milone, \& A. 1997, ApJ, 479, 902

Schlegel, D. J., Finkbeiner, D. P., \& Davis, M. 1998, ApJ, 500, 525

Schönrich, R. 2012, MNRAS, 427, 274

Searle, L. \& Zinn, R. 1978, ApJ, 225, 357

Shapley, H. 1918, ApJ, 48

Shectman, S. A. 1993, in Astronomical Society of the Pacific Conference Series, Vol. 37, Fiber Optics in Astronomy II, ed. P. M. Gray, 26 
Skrutskie, M. F., Cutri, R. M., Stiening, R., Weinberg, M. D., Schneider, S., Carpenter, J. M., Beichman, C., Capps, R., Chester, T., Elias, J., Huchra, J., Liebert, J., Lonsdale, C., Monet, D. G., Price, S., Seitzer, P., Jarrett, T., Kirkpatrick, J. D., Gizis, J. E., Howard, E., Evans, T., Fowler, J., Fullmer, L., Hurt, R., Light, R., Kopan, E. L., Marsh, K. A., McCallon, H. L., Tam, R., Van Dyk, S., \& Wheelock, S. 2006, AJ, 131, 1163

Slater, C. T., Bell, E. F., Schlafly, E. F., Jurić, M., Martin, N. F., Rix, H.-W., Bernard, E. J., Burgett, W. S., Chambers, K. C., Finkbeiner, D. P., Goldman, B., Kaiser, N., Magnier, E. A., Morganson, E. P., Price, P. A., \& Tonry, J. L. 2013, ApJ, 762, 6

Smee, S. A., Gunn, J. E., Uomoto, A., Roe, N., Schlegel, D., Rockosi, C. M., Carr, M. A., Leger, F., Dawson, K. S., Olmstead, M. D., Brinkmann, J., Owen, R., Barkhouser, R. H., Honscheid, K., Harding, P., Long, D., Lupton, R. H., Loomis, C., Anderson, L., Annis, J., Bernardi, M., Bhardwaj, V., Bizyaev, D., Bolton, A. S., Brewington, H., Briggs, J. W., Burles, S., Burns, J. G., Castander, F. J., Connolly, A., Davenport, J. R. A., Ebelke, G., Epps, H., Feldman, P. D., Friedman, S. D., Frieman, J., Heckman, T., Hull, C. L., Knapp, G. R., Lawrence, D. M., Loveday, J., Mannery, E. J., Malanushenko, E., Malanushenko, V., Merrelli, A. J., Muna, D., Newman, P. R., Nichol, R. C., Oravetz, D., Pan, K., Pope, A. C., Ricketts, P. G., Shelden, A., Sandford, D., Siegmund, W., Simmons, A., Smith, D. S., Snedden, S., Schneider, D. P., SubbaRao, M., Tremonti, C., Waddell, P., \& York, D. G. 2013, AJ, 146, 32

Steinmetz, M., Zwitter, T., Siebert, A., Watson, F. G., Freeman, K. C., Munari, U., Campbell, R., Williams, M., Seabroke, G. M., Wyse, R. F. G., Parker, Q. A., 
Bienaymé, O., Roeser, S., Gibson, B. K., Gilmore, G., Grebel, E. K., Helmi, A., Navarro, J. F., Burton, D., Cass, C. J. P., Dawe, J. A., Fiegert, K., Hartley, M., Russell, K. S., Saunders, W., Enke, H., Bailin, J., Binney, J., Bland-Hawthorn, J., Boeche, C., Dehnen, W., Eisenstein, D. J., Evans, N. W., Fiorucci, M., Fulbright, J. P., Gerhard, O., Jauregi, U., Kelz, A., Mijović, L., Minchev, I., Parmentier, G., Peñarrubia, J., Quillen, A. C., Read, M. A., Ruchti, G., Scholz, R.-D., Siviero, A., Smith, M. C., Sordo, R., Veltz, L., Vidrih, S., von Berlepsch, R., Boyle, B. J., \& Schilbach, E. 2006, AJ, 132, 1645

Strömberg, G. 1946, ApJ, 104, 12

Tonry, J. \& Davis, M. 1979, AJ, 84, 1511

van der Walt, S., Schönberger, J. L., Nunez-Iglesias, J., Boulogne, F., Warner, J. D., Yager, N., Gouillart, E., Yu, T., \& the scikit-image contributors. 2014, PeerJ, 2, e453

Vera-Ciro, C. \& Helmi, A. 2013, ApJ, 773, L4

Vogt, S. S., Mateo, M., Olszewski, E. W., \& Keane, M. J. 1995, AJ, 109, 151

Warren, S. R. \& Cole, A. A. 2009, MNRAS, 393, 272

Wegg, C., Gerhard, O., \& Portail, M. 2015, MNRAS, 450, 4050

Wilson, J. C., Hearty, F., Skrutskie, M. F., Majewski, S. R., Schiavon, R., Eisenstein, D., Gunn, J., Holtzman, J., Nidever, D., Gillespie, B., Weinberg, D., Blank, B., Henderson, C., Smee, S., Barkhouser, R., Harding, A., Hope, S., Fitzgerald, G., Stolberg, T., Arns, J., Nelson, M., Brunner, S., Burton, A., Walker, E., Lam, C., Maseman, P., Barr, J., Leger, F., Carey, L., MacDonald, N., Ebelke, G., Beland, S., Horne, T., Young, E., Rieke, G., Rieke, M., O’Brien, T., Crane, J., Carr, 
M., Harrison, C., Stoll, R., Vernieri, M., Shetrone, M., Allende-Prieto, C., Johnson, J., Frinchaboy, P., Zasowski, G., Garcia Perez, A., Bizyaev, D., Cunha, K., Smith, V. V., Meszaros, S., Zhao, B., Hayden, M., Chojnowski, S. D., Andrews, B., Loomis, C., Owen, R., Klaene, M., Brinkmann, J., Stauffer, F., Long, D., Jordan, W., Holder, D., Cope, F., Naugle, T., Pfaffenberger, B., Schlegel, D., Blanton, M., Muna, D., Weaver, B., Snedden, S., Pan, K., Brewington, H., Malanushenko, E., Malanushenko, V., Simmons, A., Oravetz, D., Mahadevan, S., \& Halverson, S. 2012, in Proc. SPIE, Vol. 8446, Ground-based and Airborne Instrumentation for Astronomy IV, 84460H

Yanny, B., Rockosi, C., Newberg, H. J., Knapp, G. R., Adelman-McCarthy, J. K., Alcorn, B., Allam, S., Allende Prieto, C., An, D., Anderson, K. S. J., Anderson, S., Bailer-Jones, C. A. L., Bastian, S., Beers, T. C., Bell, E., Belokurov, V., Bizyaev, D., Blythe, N., Bochanski, J. J., Boroski, W. N., Brinchmann, J., Brinkmann, J., Brewington, H., Carey, L., Cudworth, K. M., Evans, M., Evans, N. W., Gates, E., Gänsicke, B. T., Gillespie, B., Gilmore, G., Nebot Gomez-Moran, A., Grebel, E. K., Greenwell, J., Gunn, J. E., Jordan, C., Jordan, W., Harding, P., Harris, H., Hendry, J. S., Holder, D., Ivans, I. I., Ivezič, Ž., Jester, S., Johnson, J. A., Kent, S. M., Kleinman, S., Kniazev, A., Krzesinski, J., Kron, R., Kuropatkin, N., Lebedeva, S., Lee, Y. S., French Leger, R., Lépine, S., Levine, S., Lin, H., Long, D. C., Loomis, C., Lupton, R., Malanushenko, O., Malanushenko, V., Margon, B., Martinez-Delgado, D., McGehee, P., Monet, D., Morrison, H. L., Munn, J. A., Neilsen, Jr., E. H., Nitta, A., Norris, J. E., Oravetz, D., Owen, R., Padmanabhan, N., Pan, K., Peterson, R. S., Pier, J. R., Platson, J., Re Fiorentin, P., Richards, G. T., Rix, H.-W., Schlegel, D. J., Schneider, D. P., Schreiber, M. R., Schwope, A., Sibley, V., Simmons, A., Snedden, S. A., Allyn Smith, J., Stark, L., Stauffer, F., 
Steinmetz, M., Stoughton, C., SubbaRao, M., Szalay, A., Szkody, P., Thakar, A. R., Sivarani, T., Tucker, D., Uomoto, A., Vanden Berk, D., Vidrih, S., Wadadekar, Y., Watters, S., Wilhelm, R., Wyse, R. F. G., Yarger, J., \& Zucker, D. 2009, AJ, 137, 4377

Zhao, G., Zhao, Y.-H., Chu, Y.-Q., Jing, Y.-P., \& Deng, L.-C. 2012, Research in Astronomy and Astrophysics, 12, 723

Zinn, R. \& West, M. J. 1984, ApJS, 55, 45

Zwitter, T., Siebert, A., Munari, U., Freeman, K. C., Siviero, A., Watson, F. G., Fulbright, J. P., Wyse, R. F. G., Campbell, R., Seabroke, G. M., Williams, M., Steinmetz, M., Bienaymé, O., Gilmore, G., Grebel, E. K., Helmi, A., Navarro, J. F., Anguiano, B., Boeche, C., Burton, D., Cass, P., Dawe, J., Fiegert, K., Hartley, M., Russell, K., Veltz, L., Bailin, J., Binney, J., Bland-Hawthorn, J., Brown, A., Dehnen, W., Evans, N. W., Re Fiorentin, P., Fiorucci, M., Gerhard, O., Gibson, B., Kelz, A., Kujken, K., Matijevič, G., Minchev, I., Parker, Q. A., Peñarrubia, J., Quillen, A., Read, M. A., Reid, W., Roeser, S., Ruchti, G., Scholz, R.-D., Smith, M. C., Sordo, R., Tolstoi, E., Tomasella, L., Vidrih, S., \& Wylie-de Boer, E. 2008, AJ, 136,421 